LA--11327-MS

DE88 012950

Initial Borehole Acoustic Televiewer

Data Processing Algorithms

Troy K. Moore

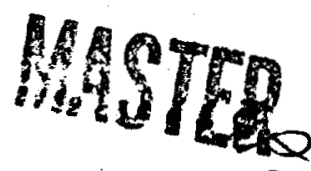

DISTRIBUTION OF THIS DOCUMENT IS UNLIMITEO

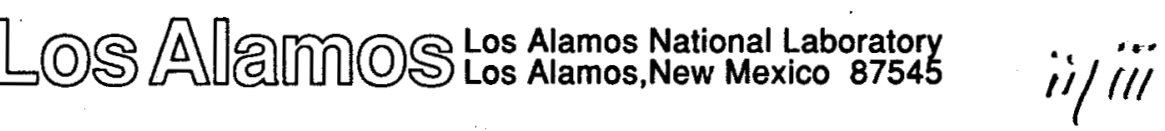




\section{DISCLAIMER}

This report was prepared as an account of work sponsored by an agency of the United States Government. Neither the United States Government nor any agency Thereof, nor any of their employees, makes any warranty, express or implied, or assumes any legal liability or responsibility for the accuracy, completeness, or usefulness of any information, apparatus, product, or process disclosed, or represents that its use would not infringe privately owned rights. Reference herein to any specific commercial product, process, or service by trade name, trademark, manufacturer, or otherwise does not necessarily constitute or imply its endorsement, recommendation, or favoring by the United States Government or any agency thereof. The views and opinions of authors expressed herein do not necessarily state or reflect those of the United States Government or any agency thereof. 


\section{DISCLAIMER}

Portions of this document may be illegible in electronic image products. Images are produced from the best available original document. 



\section{Table of Contents}

FIGURES and TABLE

vii

ABSTRACT

I. Introduction

A. Motivation

B. Basic Assumptions

1

1

1

II.BDCOMP (BAT Data Complement and Fix)

A. Functional Description 3

B. Implementation 3

C. BDCOMP User's Guide 5

III. DATASEP (BAT Data Separation) 5

$\begin{array}{lr}\text { A. Functional Description } & 5 \\ \text { B. Implementation } & 5 \\ \text { C. DATASEP User's Guide } & 16\end{array}$

IV. DPDUMP (DP File Dump) 16

$\begin{array}{ll}\text { A. Functional Description } & 16 \\ \text { B. Implementation } & 16 \\ \text { C. DPDUMP User's Guide } & 16\end{array}$

V. BDINVRT (BAT Data Invert Utility) 18

A. Functional Description 18

B. Implementation $\quad 18$

$\begin{array}{ll}\text { C. BDINVRT User's Guide } & 18\end{array}$

VI. BDEXTRCT (BAT Data Extraction Utility) 18

A. Functional Description $\quad 18$

B. Implementation $\quad 18$

C. BDEXTRCT User's Guide 21

VII. BATD (BAT Display Utility) $\quad 21$

A. Functional Description $\quad 21$

B. Implementation $\quad 21$

C. BATD User's Guide $\quad 26$

1. Menu Description $\quad 26$

2. Selecting Records 31

3. Logical Display 31

VIII. BATT (BAT Trace Generation Utility) $\quad 34$

A. Functional Description $\quad 34$

B. Implementation $\quad 34$

C. BATT User's Guide $\quad 38$

1. Menu Description 38

2. Display Definition $\quad 42$

3. Usage Notes 47

$\begin{array}{ll}\text { REFERENCES } & 47\end{array}$ 


\section{Table of Contents}

APPENDICES

A. Workstation Development Environment 49

B. Disk Files Used to Generate Algorithms $\quad 50$

C. Field Tape Format $\quad 51$

D. Typical Terminal Session Output 53

E. BDCOMP Source Code $\quad 55$

F. DATASEP Source Code $\quad 59$

G. DPDUMP Source Code 77

H. BDINVRT Source Code $\quad 81$

I. BDEXTRCT Source Code $\quad 85$

J. BATD Source Code $\quad 90$

K. BATT Source Code 138 


\section{Figures}

Figure 1. Typical Processing Path 2

Figure 2. BDCOMP Flowchart 4

Figure 3. Files Produced by DATASEP 6

$\begin{array}{lll}\text { Figure } 4 . & \text { DATASEP Flowchart } & 7\end{array}$

$\begin{array}{llll}\text { Figure } & 5 . & \text { file_prep Flowchart } & 9\end{array}$

$\begin{array}{lll}\text { Figure 6. name_gen Flowchart } & 10\end{array}$

$\begin{array}{lll}\text { Figure 7. find_first_rec Flowchart } & 10\end{array}$

Figure 8. find_next_rec Flowchart 11

Figure 9. get_depth Flowchart 12

Figure 10. save_log_heads Flowchart 12

Figure 11. Log Header File Format 13

Figure 12. Data File Format 14

Figure 13. DP File Format 15

$\begin{array}{lll}\text { Figure 14. DPDUMP Flowchart } & 17\end{array}$

$\begin{array}{lll}\text { Figure } & 15 . & \text { BDINVRT Flowchart }\end{array}$

Figure 16. BDEXTRCT Flowchart 20

Figure $17 . \quad$ BATD Flowchart 22

Figure 18. rec_sel_rec1 Flowchart 24

Figure $19 . \quad$ rec_sel_rec2 Flowchart 25

Figure 20. depth_sel_rec Flowchart 27

Figure 21. disp_recs Flowchart 28

$\begin{array}{lll}\text { Figure } 22 . & \text { BATD Menu Structure } & 29\end{array}$

Figure 23. Example of Display Generated by BATD 32

Figure 24. Parameters Used to Generate Figure $23 \quad 33$

Figure 25. BATT Flowchart 35

Figure 26. select_recs Flowchart 37

Figure 27. intrvl_calc Flowchart 39

Figure 28. calc_tick_lines Flowchart 40

Figure 29. gen_disp Flowchart 41

Figure $30 . \quad$ BATT Menu Structure 43

Figure 31 . Typical Traces Generated by BATT 43

Figure 32. Parameters Used to Generate Figure 31

Figure 33. Example of Two Traces with Associated Tick Lines 45

Table

Table I. Tick Line Representations 


\title{
Initial Borehole Acoustic Televiewer Data Processing Algorithms
}

by

\author{
Troy K. Moore
}

\begin{abstract}
With the development of a new digital televiewer, several algorithms have been developed in support of off-line data processing. This report describes the initial set of utilities developed to support data handling as well as data display. Functional descriptions, implementation details, and instructions for use of the seven algorithms are provided.
\end{abstract}

\section{Introduction}

\section{A. Motivation}

Recently, a new borehole acoustic televiewer has been developed jointly by Westfalische Berggewerkschaftskasse (WBK) of Bochum, FRG, and Los Alamos National Laboratory.1,2 This tool records digital data collected in the field on $1 / 4$-in. data cartridges. Once the field data have been read into a processing workstation, various mission-specific processing algorithms may be applied.

This report documents the algorithms developed for initial off-line processing of Borehole Acoustic Televiewer (BAT) data. The function and implementation of each algorithm are described. A user's guide for each is also included. Figure 1 illustrates the processing paths provided by the utilities described herein. Algorithms are discussed in the order they could be encountered in a processing scenario.

\section{B. Basic Assumptions}

Several basic assumptions and preconditions should be defined before examining each utility. These include the following:

1. This document is intended to be an overview of the currently existing utilities. Development environment details are contained in Appendices A - F. For exact details such as passed parameters, variable names, etc., the user is directed to the in-code documentation. See Appendices E - K. 


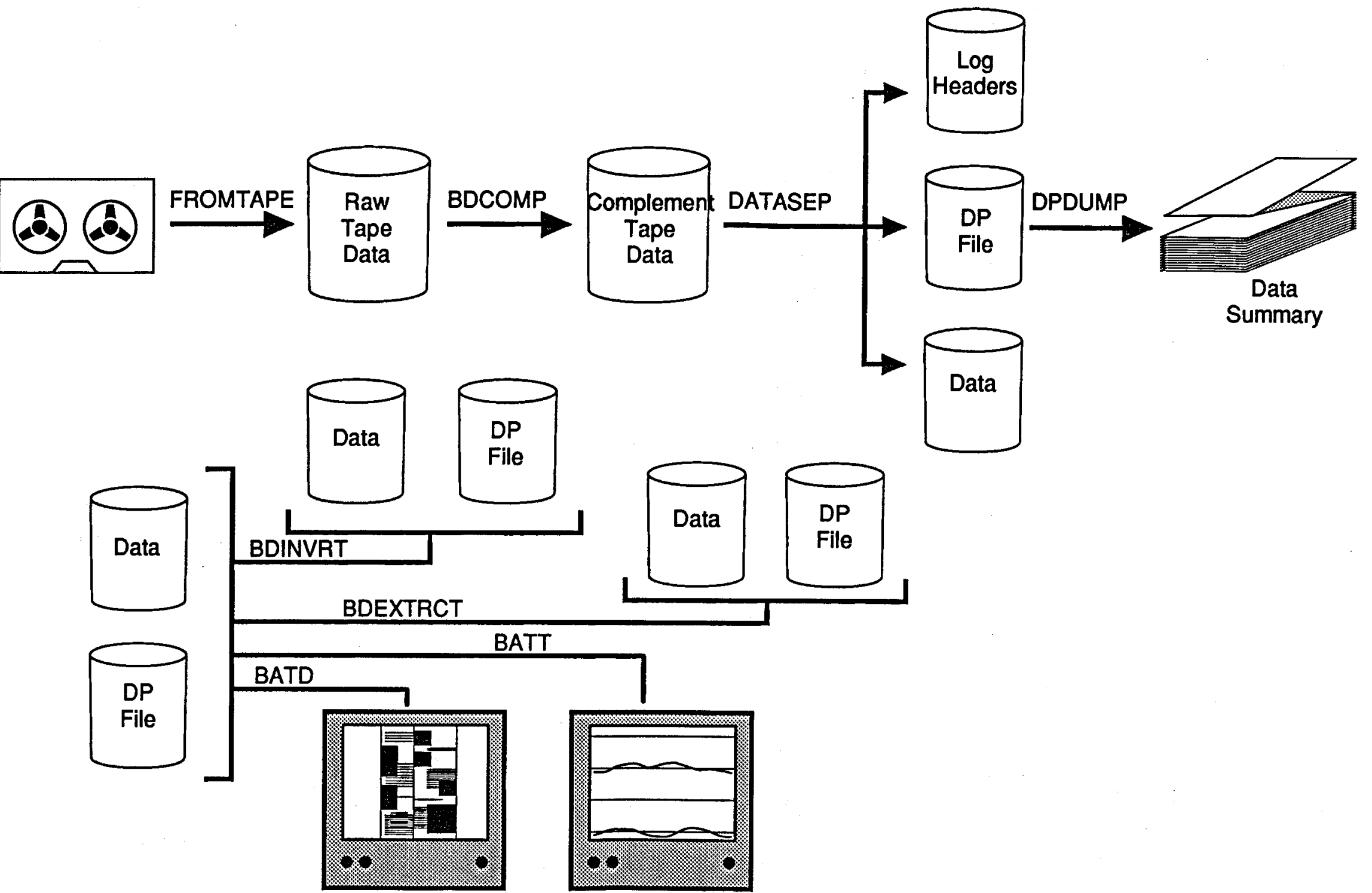

Figure 1. Typical processing path. 
2. Console refers to the CRT used as the primary output device connected to the CPU, whereas display is the monitor attached to the imaging board set.

3. Unless overridden, data from shallower portions of the area of interest will be displayed at the top of the display. As the eye travels down the display, data from deeper in the wellbore will be encountered.

4. When using BATD and BATT, all data are assumed to have been collected as the tool is extracted from the wellbore. This will be considered the standard data format.

5. In all descriptions except that for BATT, the terms travel time and caliper can be used interchangeably.

6. Offsets begin with 0 ; record numbers start with 1 .

7. The tape transfer utility FROMTAPE will not be discussed in this report. This utility was purchased and has its own documentation.

8. Utilities described in the report are still being developed; they may change--and contain errors.

\section{BDCOMP (BAT Data Complement and Fix)}

\section{A. Functional Description}

The initial data collection of April 13,1987, used downhole and uphole software that contained two unique features:

- the $S$ in the log header keystring Sabis Field Tape was replaced with $00 \mathrm{~h}$, and

- all data were complemented before being written to tape.

BDCOMP was developed to complement the data read from the field tapes and replace the missing $S$. Actual field tape format is shown in Appendix C.

\section{B. Implementation}

BDCOMP was written as a single function designed to process one buffer of data at a time. See Figure 2 for a detailed flowchart. Each byte not equal to $0 \mathrm{FFh}(0 \mathrm{~h}$ complemented) is automatically complemented. Any $0 F F$ bytes are treated as though they may represent a missing $S$. To determine where the $S$ should be inserted, a four-character subkeystring following the current buffer position is examined. If this subkeystring contains the complement of abis, then an $S$ is inserted in the current position. Even though this is not an absolute test (an absolute test would require matching the entire subkeystring abis Field Tape), the existence of OFFh followed by the complement of abis is sufficient evidence to insert the missing character.

Note that future versions of the uphole software will most likely have the $S$ removal bug corrected. BDCOMP will work correctly in either case as long as the data still need to be complemented.

Implementation is straightforward except for identifying a potential substring that is not entirely contained within the buffer. In this case, the file pointer must be manipulated in preparation for the next read operation. Statistical information provided at the termination of BDCOMP indicates the number of file repositions required as well as the actual number of $S$ 's inserted. 


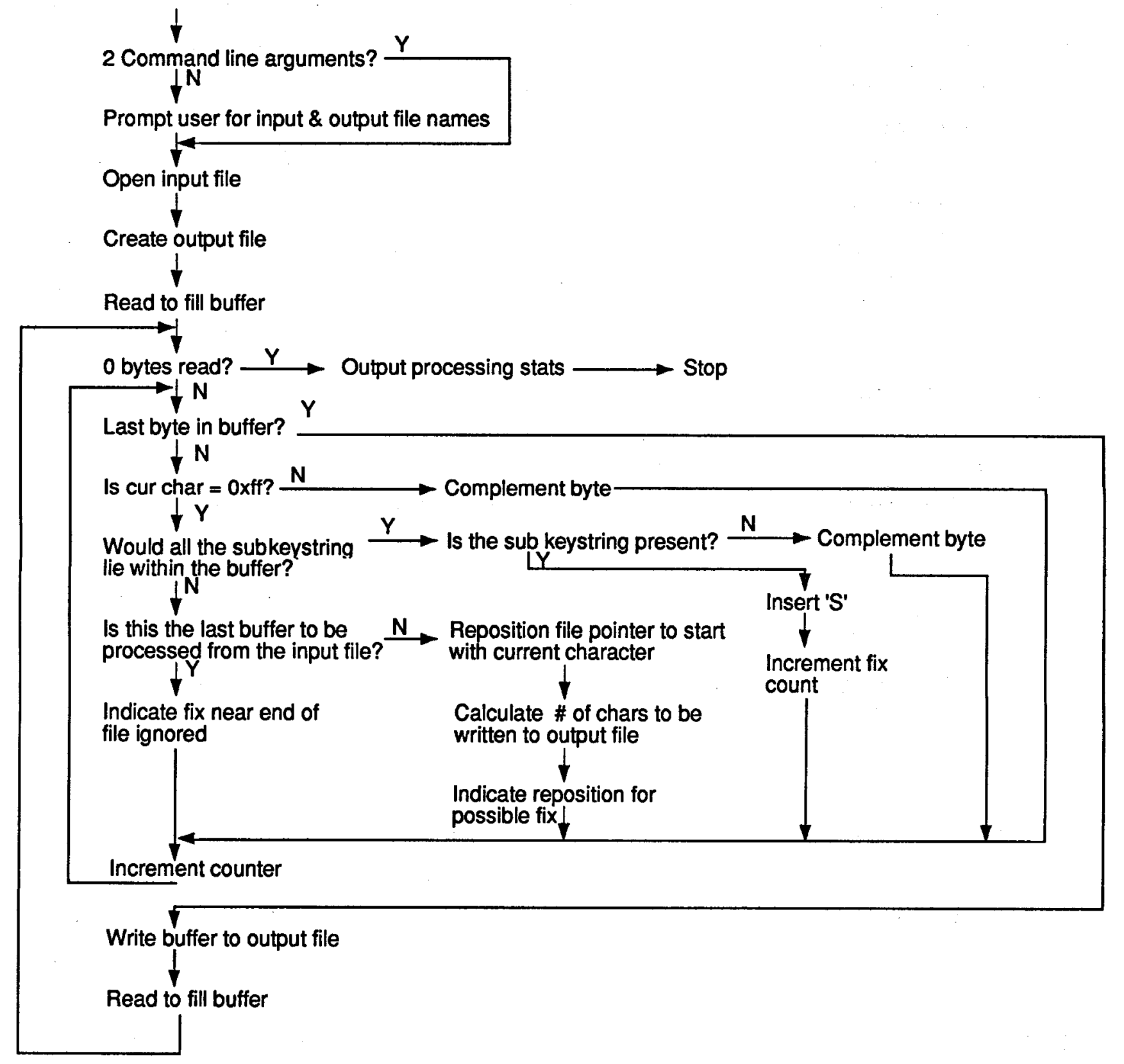

Figure 2. BDCOMP flowchart. 


\section{BDCOMP User's Guide}

Input file and output file specifications are the only parameters required for operation. These parameters may be entered by the command line or in response to run-time prompts. Drive and path specifications are supported.

The command line syntax is as follows:

$$
\text { BDCOMP input_file output_file }
$$

\section{DATASEP (BAT Data Separation)}

\section{A. Functional Description}

DATASEP is designed to separate a corrected raw tape data file (post-BDCOMP processing) into three separate files. This operation is included to ease data handling in subsequent processing. The first file produced represents all $\log$ header blocks located within the tape file. A second file contains only revolution data (revolution header, amplitude, and travel time data). The third file, referred to as the data pointer (DP) file, provides random access to data contained within the second file. See Figure 3.

\section{B. Implementation}

DATASEP classifies a sequence of bytes from the input file as either a log header record or a revolution data record based on keystrings. These keystrings are Sabis Log Header for log header records or Sabislog for revolution data. Once a keystring has been located, the next keystring is found to determine record length. A highlevel flowchart is shown in Figure 4.

DATASEP is implemented as a moderately complex main routine calling six functions. All definitions and functions are contained within the file DATASEP.C. Each function is described as follows:

\section{1. file_prep}

Obtain the input file name from the user. A set of candidate output file names is generated. If unacceptable, the user can enter new output file names. All files are then opened or created.

2. name_gen

Called by file_prep to generate default output file names.

3. find_first_rec

Used at startup to find the first record in the input file. This function calls find_next_rec.

4. find_next_rec

Find and classify the next keystring in the buffer. When this information is used, the end of the current record, start of the next record, and type of next record can be determined.

5. get_depth

Generates a floating point depth value from the ASCII depth string in the revolution data header. 


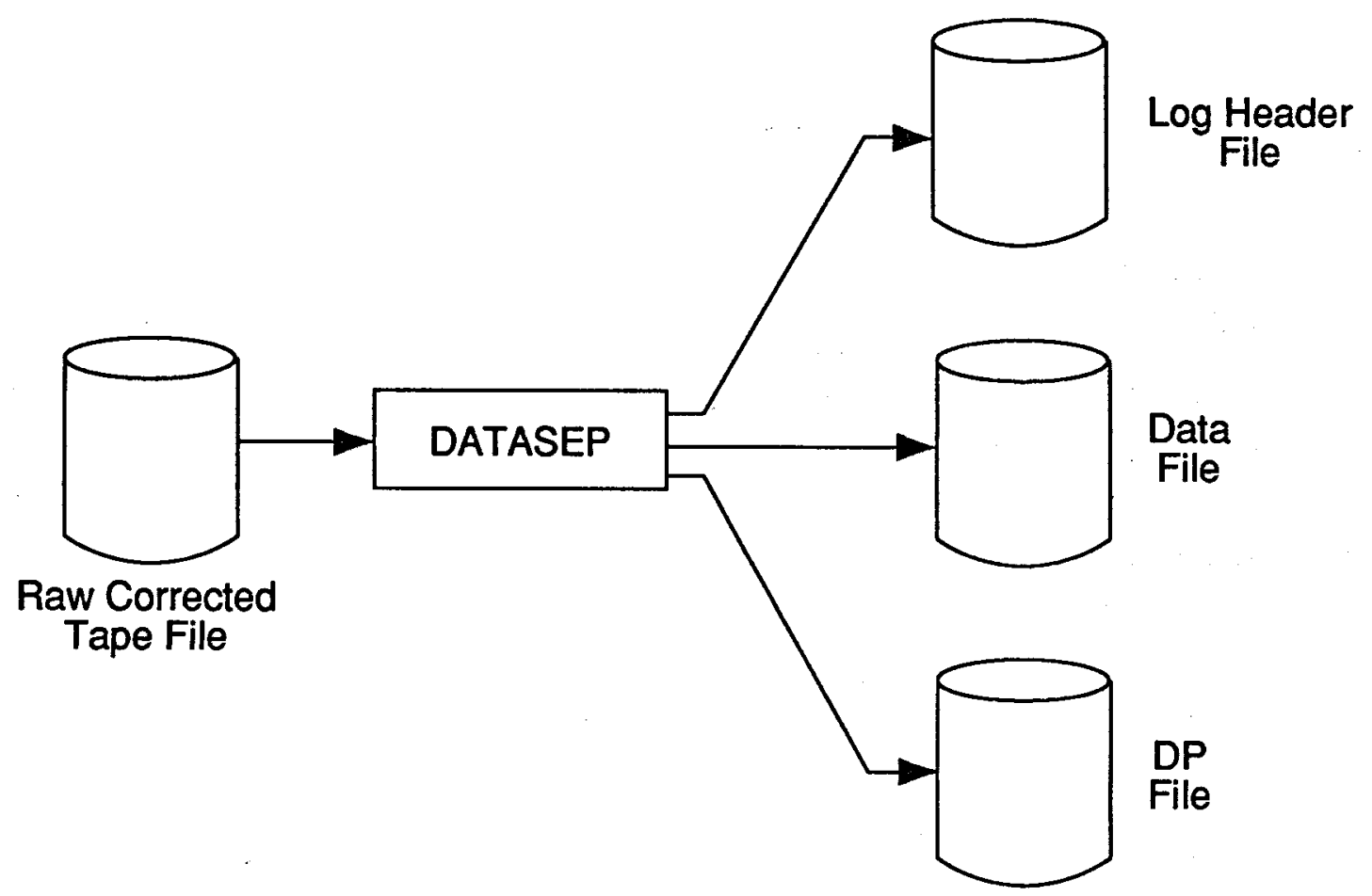

Figure 3. Files produced by DATASEP. 


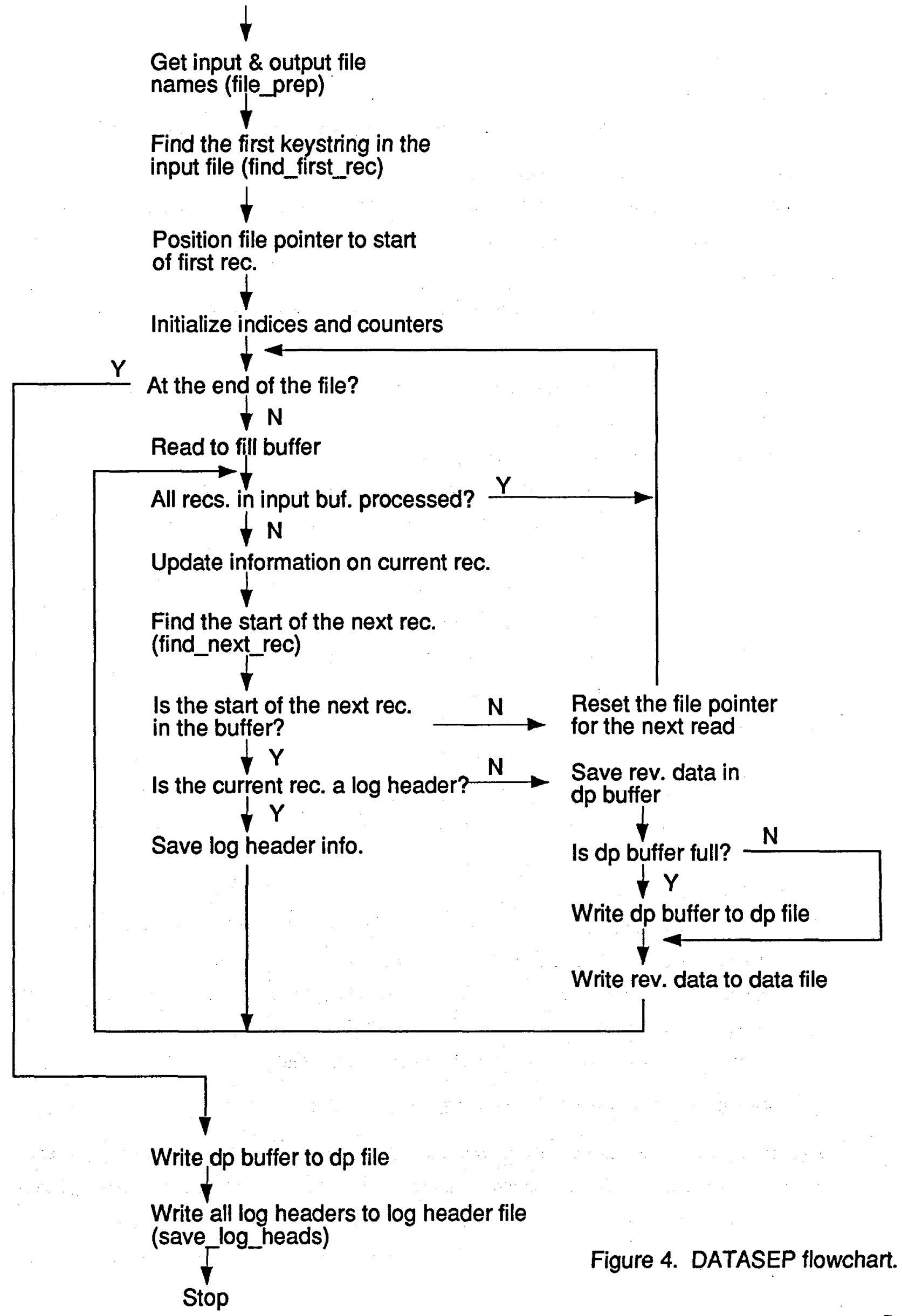


6. save_log_heads

Writes $\log$ header records and associated location and length information to the log header file.

Figures 5 - 10 contain flowcharts for each function.

Several pointers and indices are used throughout DATASEP to keep track of the current location in the input buffer as well as several data structures. The names and uses of these variables are documented within the code. See Appendix F.

DATASEP generates three output files, each with a unique format. These files are discussed below.

1. Log header file

This file will contain all the log header records found while processing the input file. It is possible to have several different log headers in a single tape file.

To determine where in the input file the log header record was located and to know how long the log header record is, six bytes of information are appended to the start of each header record. An offset (long int) relative to the start of the input file is contained within the first four bytes of each log header record. The remaining two bytes (int) represent the actual length of the log header record in the input file. When existing WBK software is used, this length is 1024 bytes. Therefore, the length of each record in the log header file should be

$\log$ header length + appended info $=1024+6=1030$ bytes.

The default file extension for the log header file is LHF. See Figure 11.

\section{Data file}

This file is similar to the input file format except that all the log header information has been removed. Any gaps produced by removing log headers are filled by sliding the revolution data forward. See Figure 12. No additional information is appended.

The default file extension for this file is RAW.

3. Data pointer file

The DP file provides random access to any revolution data record found in the data file. Each record in this file contains three entries. For the $n^{\text {th }}$ entry in the DP file:

record offset - byte offset from the start of the data file to the start of the $n^{\text {th }}$ revolution (long int) depth - depth at which revolution $\mathrm{n}$ was collected (float)

length - number of bytes in revolution data record $\mathbf{n}$ (int)

DPF is the default file extension for the DP file. See Figure 13.

Log header and revolution data records are written to their respective output files in vastly different ways. Each revolution data record is written to the data file as it is encountered. If there are no more than a few log header records per input tape file, the log header records are queued until all main control loop processing is complete. At that time, all log header records are appended auxiliary information and are written to the output file. The 


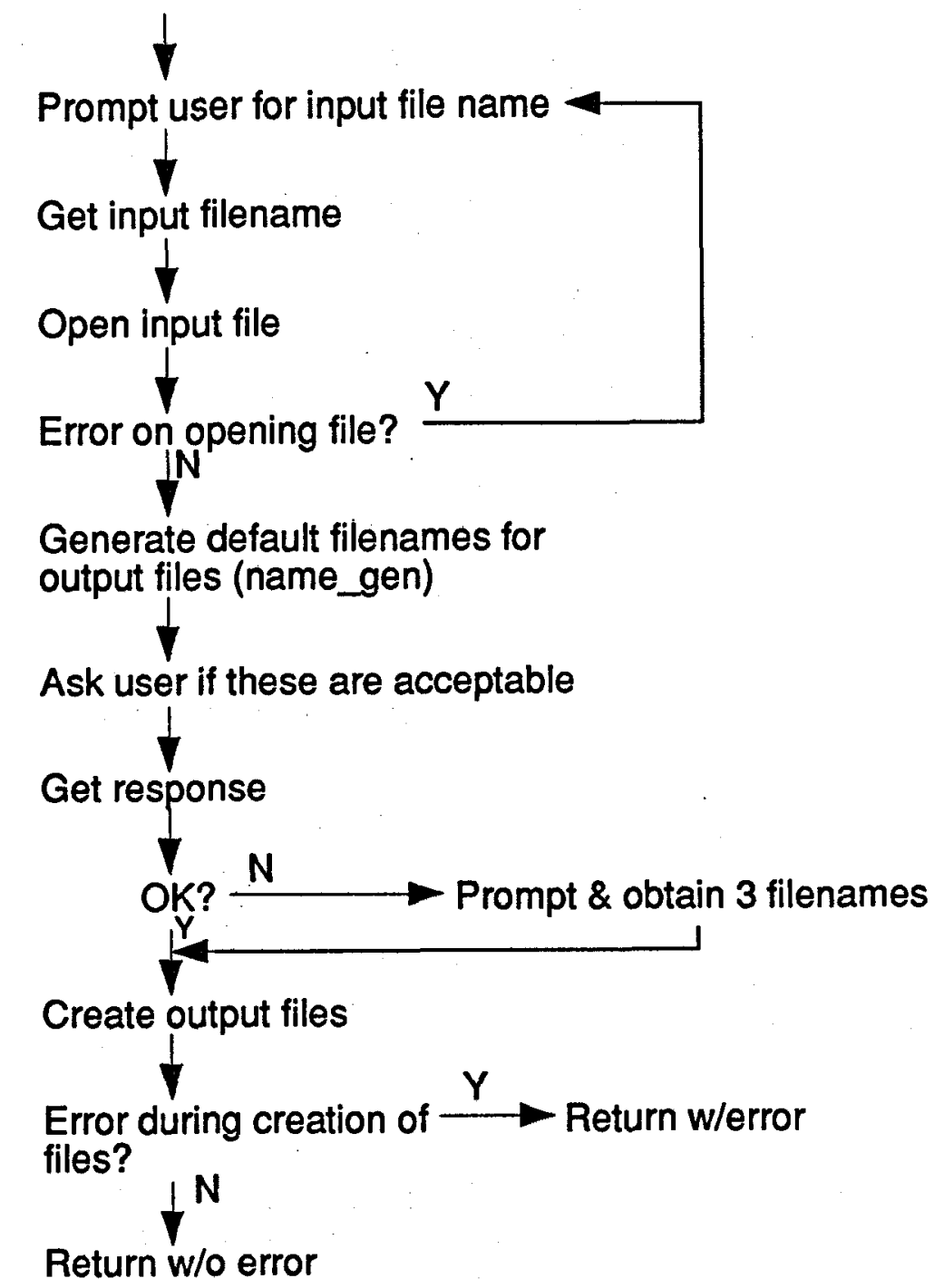

Figure 5. file_prep flowchart. 


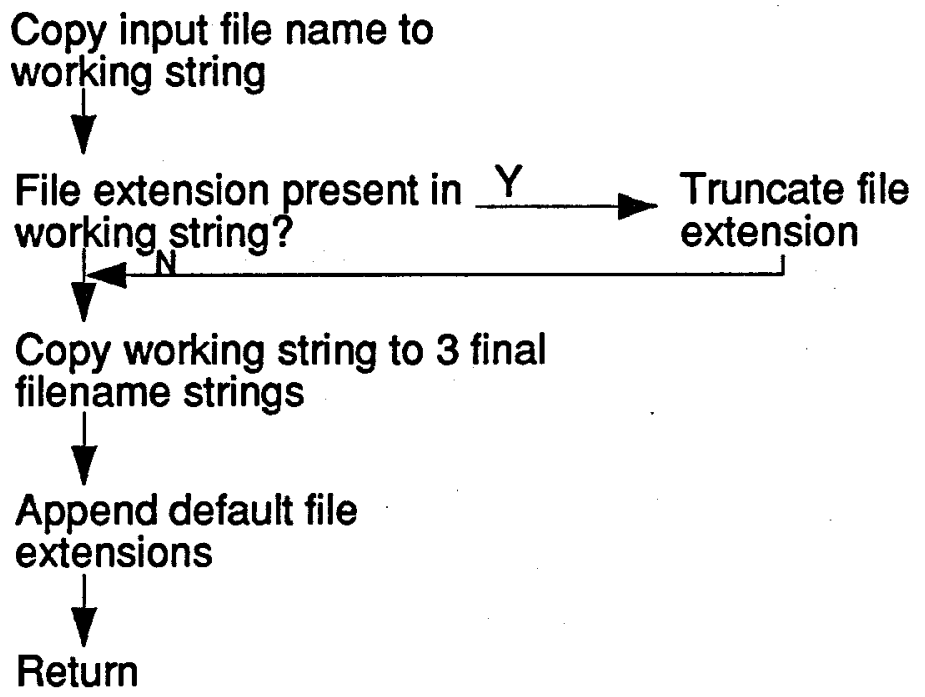

Figure 6. name_gen flowchart.

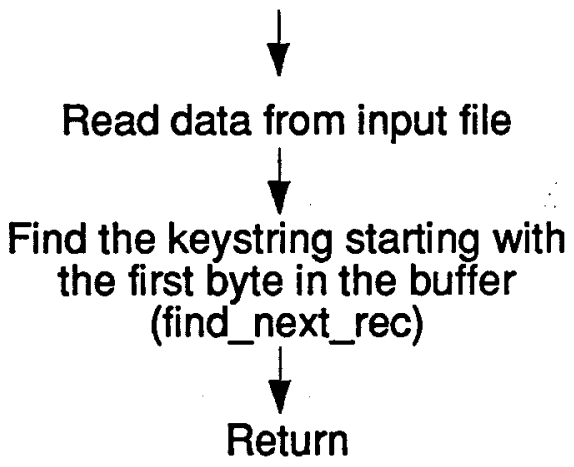

Figure 7. find_first_rec flowchart. 


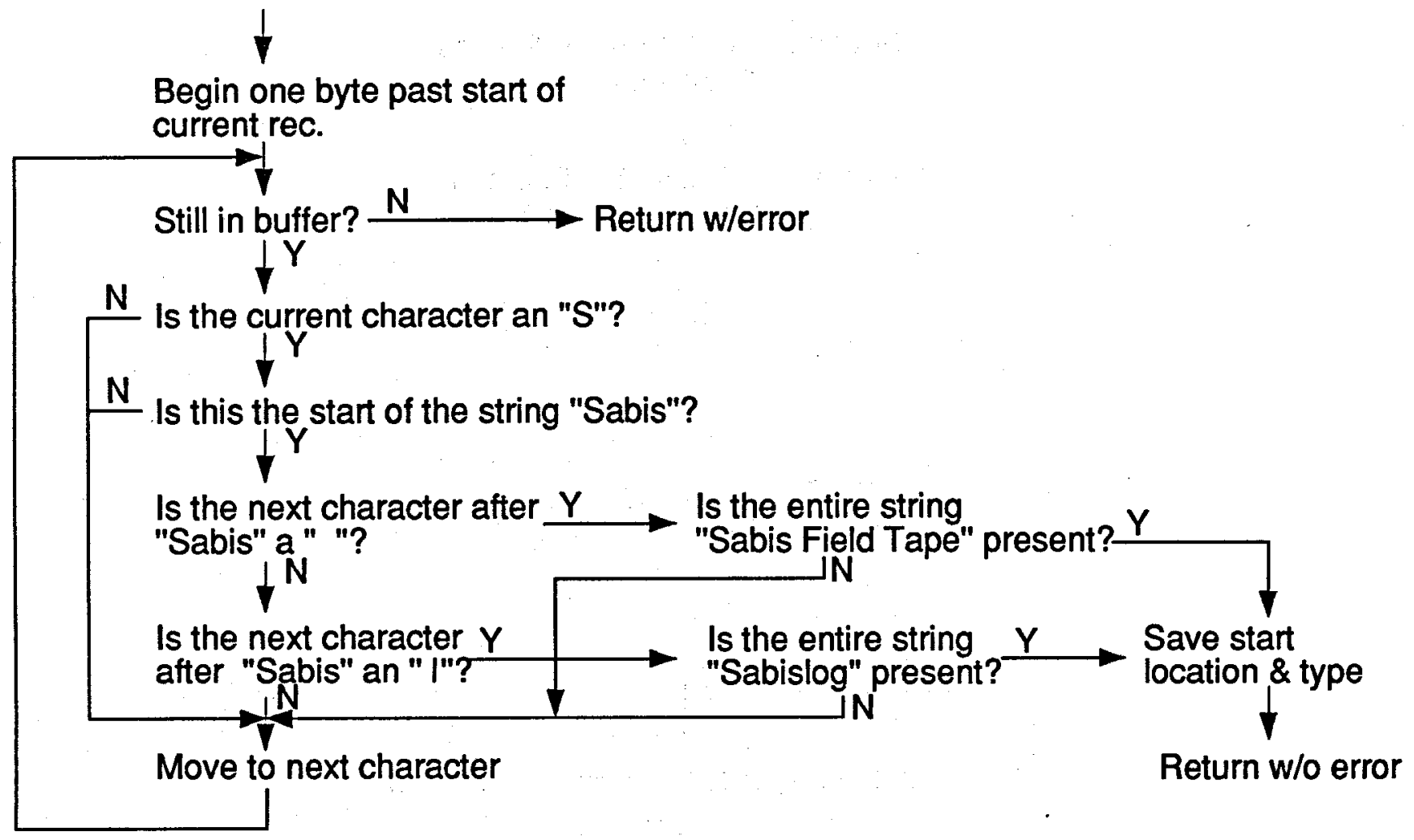

Figure 8. find_next_rec flowchart. 


$$
1
$$

Copy depth string from revolution header to working string<smiles>C1C2CC1C2</smiles>

Convert working string to floating point value<smiles>C[In]1CC1</smiles>

Insert decimal point by dividing by appropriate power of 10<smiles>C1CC12CC2</smiles>

Return

Figure 9. get_depth flowchart.

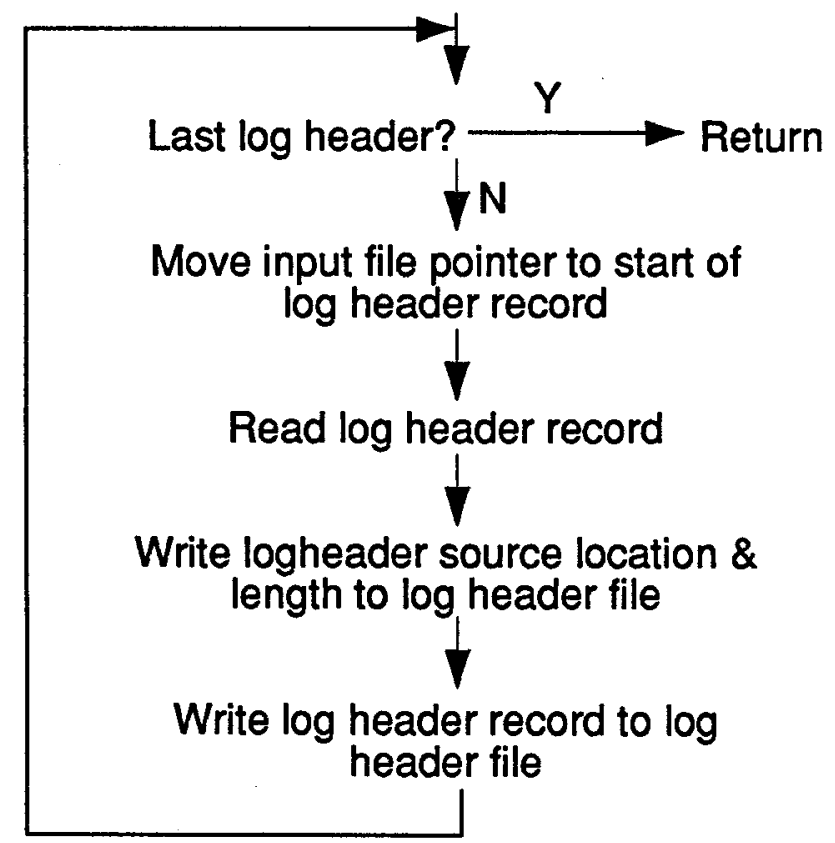

Figure 10. save_log_heads flowchart. 


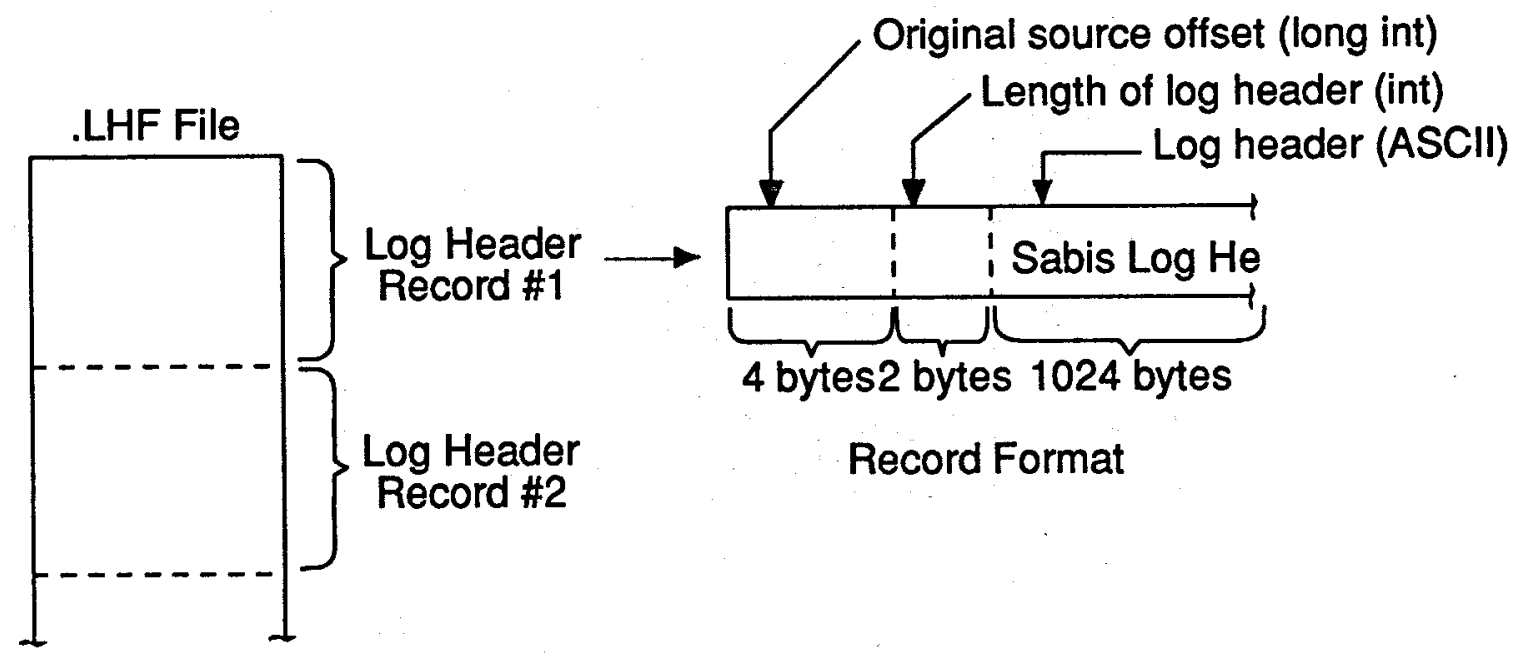

Figure 11. Log header file format. 

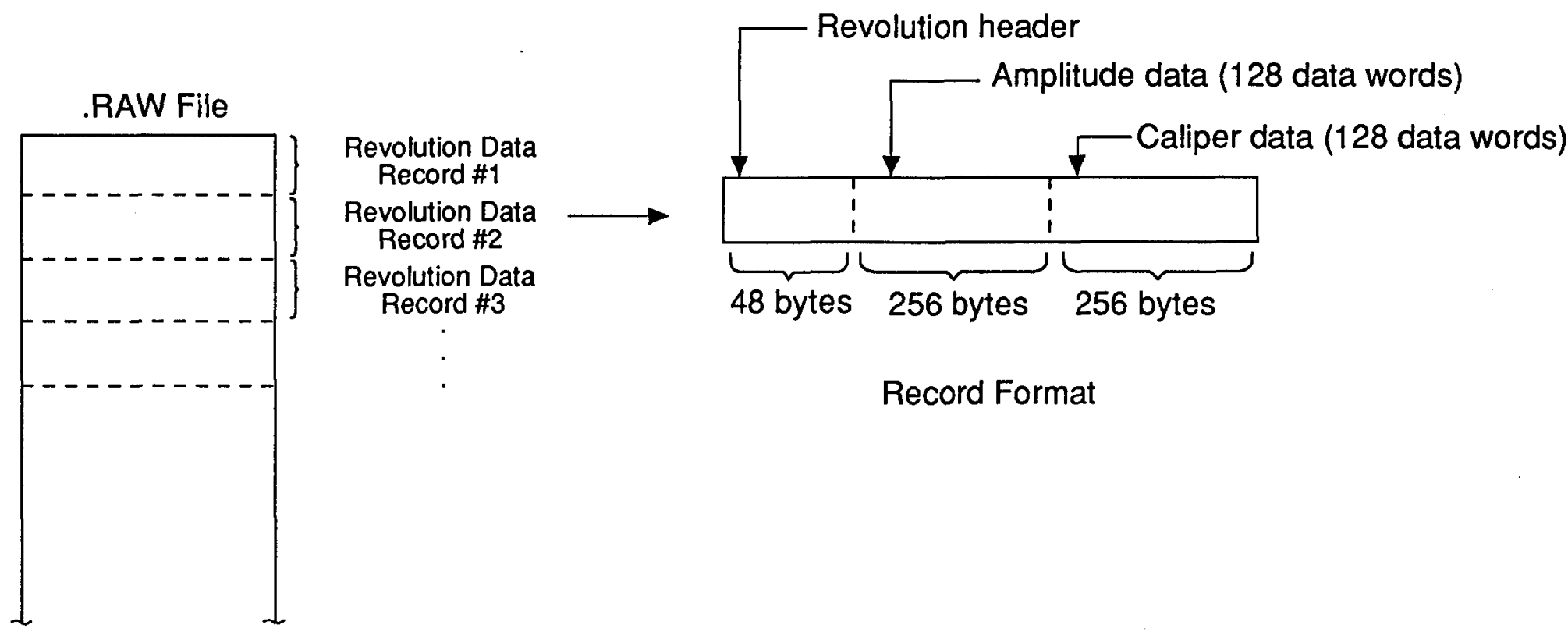

Record Format

Figure 12. Data file format. 


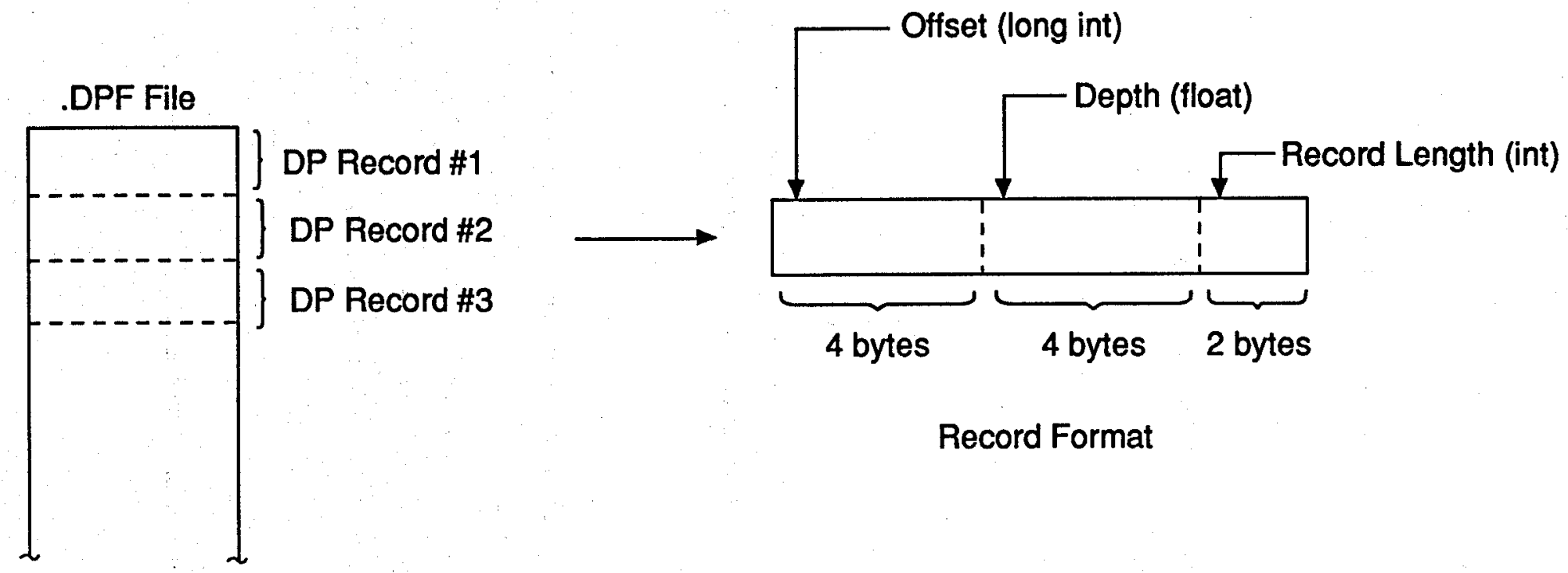

Figure 13. DP file format. 
possibility that the number of $\log$ headers may exceed the queue size does exist, but it is extremely unlikely. DATASEP (Version 1.11) allows 64 queued log headers.

As DATASEP is terminating execution, a processing summary is output to the console. See Appendix D. This summary includes the number of $\log$ headers and revolution data records identified. Note that the number of records may be one less than expected because the final record in the file does not have a keystring following it for determining the end of the record.

\section{DATASEP User's Guide}

If the user accepts the algorithm's default output files, the only user input is the input file name. Overriding the default output names is accomplished by striking any key other than Enter, which allows entry of three different user-specified filenames with any file extension.

\section{DPDUMP (DP File Dump)}

\section{A. Functional Description}

Once the data and DP files have been generated, it is convenient to summarize data file contents. The utility DPDUMP has been developed to meet this need. This utility prints the offset, depth, and length of each data file record as found in the DP file. For reference, a record number is generated and output with each DP file entry. In addition, records that are too long (not within a user-specified range) are flagged as possibly invalid. Figure 14 contains a high-level flowchart.

\section{B. Implementation}

The implementation of this utility is straightforward and simple enough that everything is handled within the main function. Output is produced in a format suitable for the printer even though the data stream is sent to stdout. Records that are too long (not within the specified range) are appended an * when output.

Output is generated on a page-by-page basis. Because there are two columns of information per page, handling the final page is the only concern. If possible, the first column will be filled completely before starting on the second.

\section{DPDUMP User's Guide}

DPDUMP is a command line arguments-only interface requiring three parameters. The syntax is as follows:

$$
\text { DPDUMP input_file base deviation }
$$

The base and deviation are used in identifying records whose length does not meet the following condition:

$$
\text { base }- \text { deviation }<=\text { record length }<=\text { base }+ \text { deviation }
$$




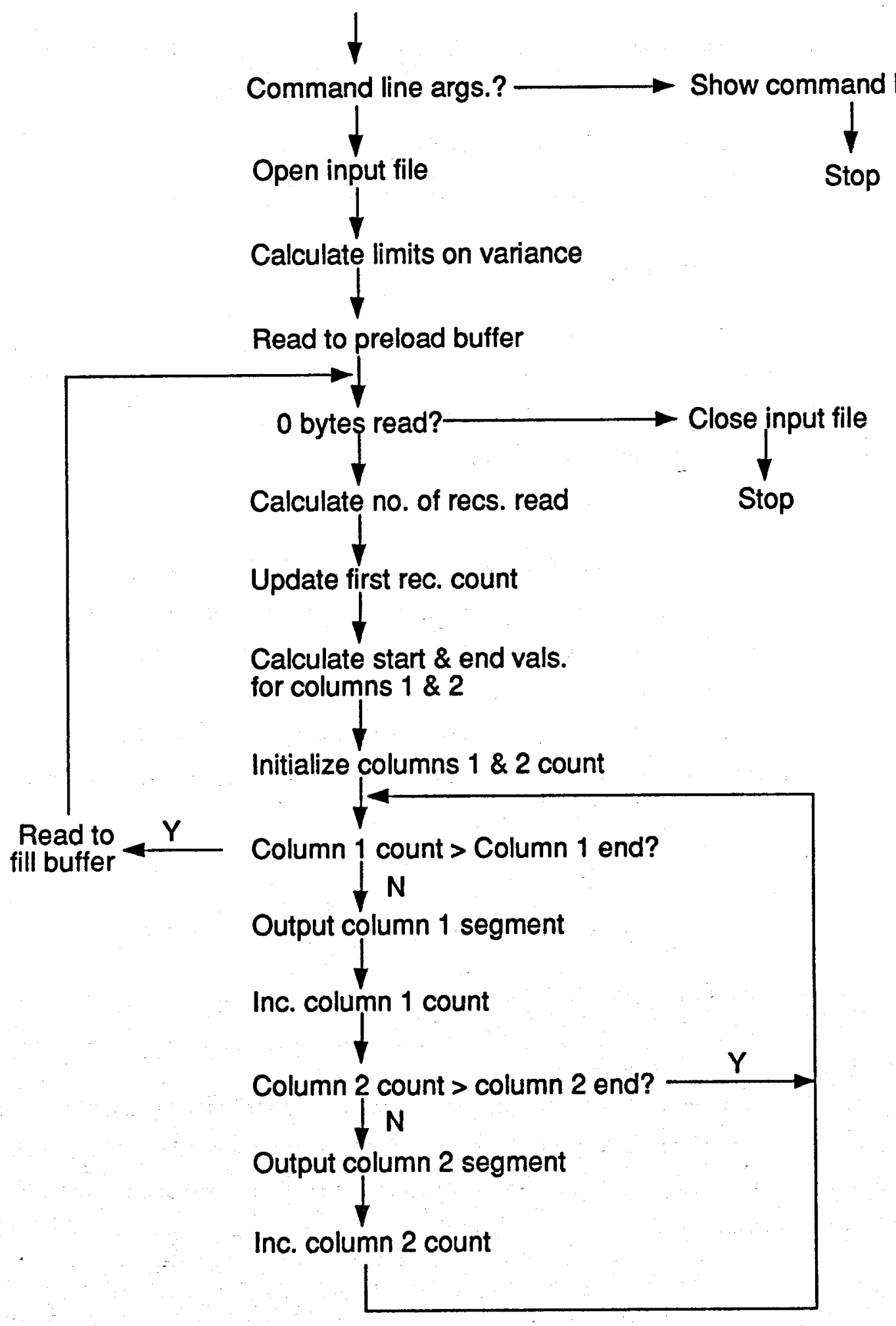

Figure 14. DPDUMP flowchart. 
As previously indicated, the output produced by DPDUMP is suitable for output to the printer even though it, by default, is sent to the console. To redirect the output to the printer, use the following syntax:

DPDUMP input_file base deviation $>$ PRN

Typical output produced by DPDUMP can be seen in Appendix $D$.

\section{BDINVRT (BAT Data Invert Utility)}

\section{A. Functional Description}

As indicated in Section I.B., it is assumed that data are always collected as the tool is extracted from the wellbore. This assumption may not always be true. BDINVRT was developed to invert data collected as the tool is lowered into the borehole into the standard data format.

\section{B. Implementation}

BDINVRT is an interactive algorithm implemented as a single function. A set of DP and raw data files is used as input. New DP and data files are produced that contain the original data but in an inverted order. The first revolution record is now the last. Data are processed on a revolution-by-revolution basis starting with the last revolution in the input file set. See the Figure 15 flowchart.

The record offsets in the new DP file are referenced to the offset from the start of the first byte in revolution records found in the new raw data file. The accelerometer data found in the revolution header are not modified during execution.

\section{BDINVRT User's Guide}

BDINVRT prompts the user for input. Only the input and output DP and raw data file names are required.

\section{BDEXTRCT (BAT Data Extraction Utility)}

\section{A. Functional Description}

During data collection, there are times when the tool is stationary or the data may be invalid. Data acquired during such periods do not contain any additional information. Because a smaller data set results in increased processing speed, data of the greatest interest should be extracted from the original data set. BDEXTRCT has been developed to meet this need.

\section{B. Implementation}

BDEXTRCT is an interactive algorithm implemented as a single function. An existing DP and raw data file set is used as input. A new set of DP and data files is produced that is a subset of the input. The user is prompted for input files, output files, and first and last records specification. Upon positioning the DP file pointer at the first requested record entry, data are processed on a revolution-by-revolution basis until the final requested record is reached. See Figure 16. 


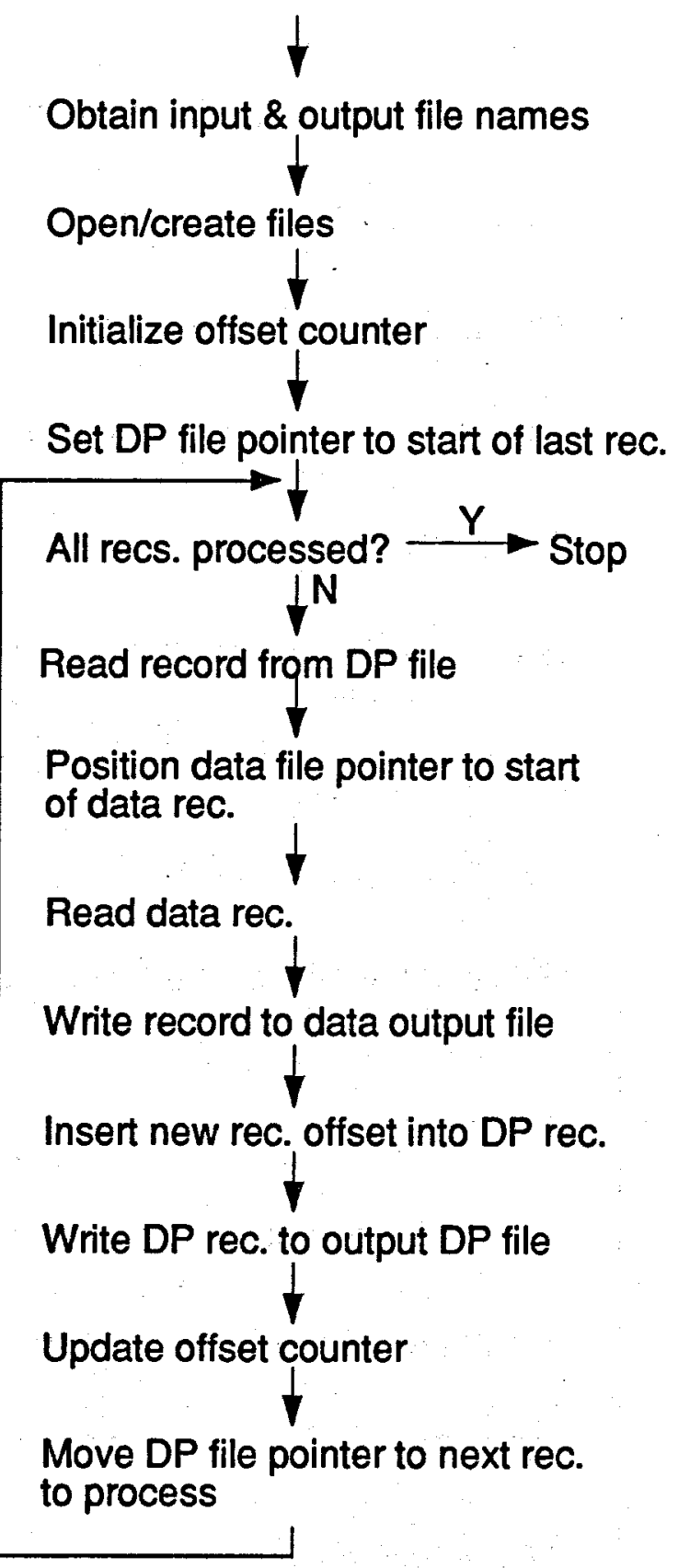

Figure 15. BDINVRT flowchart. 


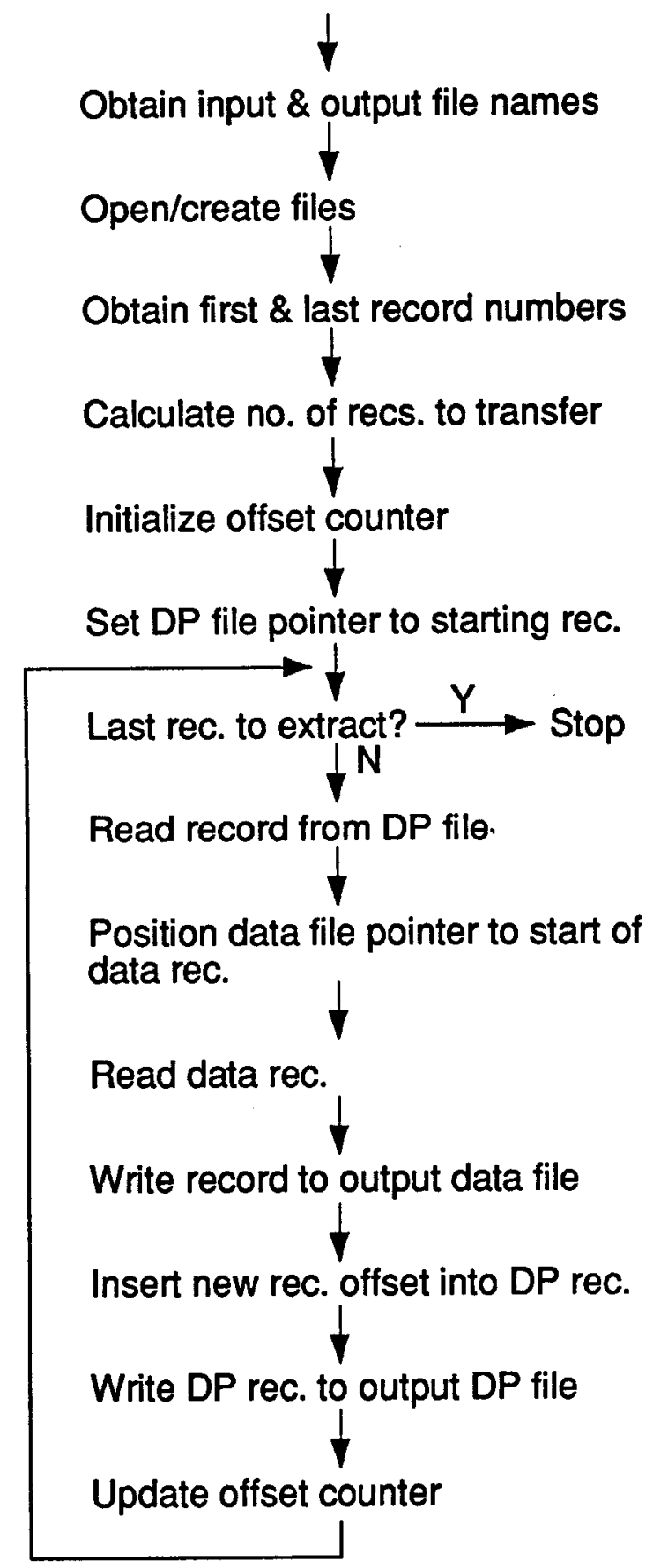

Figure 16. BDEXTRCT flowchart. 
The record offsets in the new DP file are referenced to the first byte of revolution records found in the new raw data file.

\section{BDEXTRCT User's Guide}

Three sets of information must be specified by the user. The initial input required is the DP and raw data file from which the extraction will occur. The second input specifies the file names of the files to be created. Initial and final records to be extracted represent the final data required. Records are referenced beginning with 1.

\section{BATD (BAT Display Utility)}

\section{A. Functional Description}

BATD is a utility for displaying BAT tool data. Either amplitude, or travel time, or both may be displayed. Scale factors for each type of data are specified by the user. The output may be displayed either in Channel $\mathbf{0}$ or in Channel 1 of the image memory. The user can specify where output is to be positioned on the display. Records to be displayed may be selected on a record or depth basis. Two methods are available for specifying the range of records to be selected. Extensive parameter checking helps the user to enter only valid parameters.

This utility is menu driven with several levels of pull-down submenus to provide the user with a friendly interface. Data forms ${ }^{3}$ are used to obtain parameters from the user. A status window is constantly updated to provide the user with current parameters. Any additional information or error messages are relayed to the user through windows. 4

\section{B. Implementation}

BATD is a complex algorithm simplified by the extensive use of functions. The main routine is predominantly a collection of function calls, which break the complex algorithm up into more understandable subtasks. Figure 17 provides an overview of the algorithm.

Two special libraries of functions were used to develop this code. The first, Windows for Data, Vermont Creative Software, was used to design/implement the menu structure. This library was also used to provide informational windows as well as data forms for entering data. Access to the image processing boards was provided by the DT-IRIS library from Data Translation. 5

To avoid passing numerous parameters between functions, global variables are used extensively. Information pertaining to these variables as well as to all the \#defines is contained in a set of include files. A directory of all files used is included in Appendix $B$.

To provide insight into the concepts involved in the implementation of this algorithm, each of the functions developed for BATD will be discussed in the following pages. Flowcharts are provided for the more involved functions.

\section{1. init_parms}

Initializes many of the global parameters to default values. 


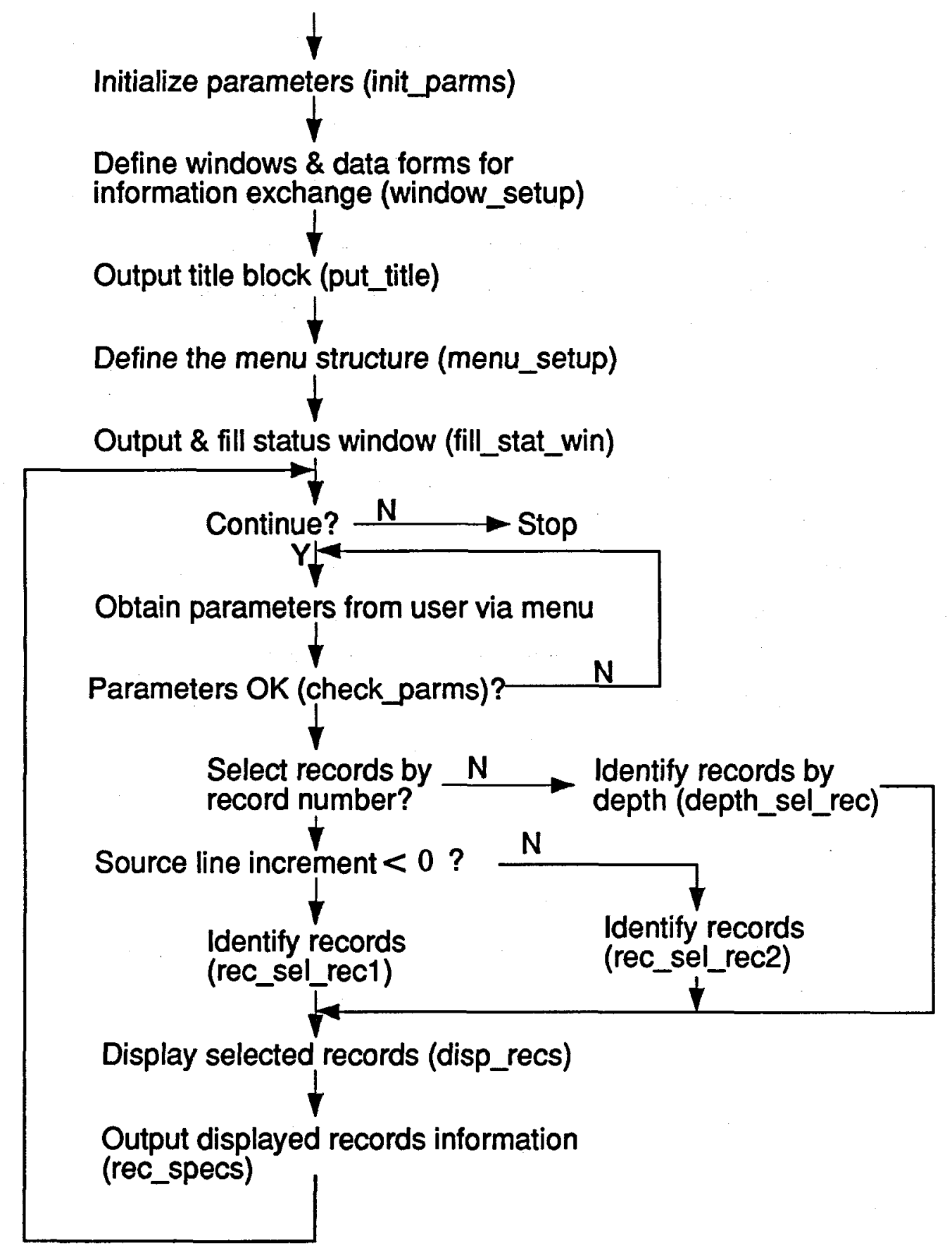

Figure 17. BATD flowchart. 
2. window_setup

Defines the windows and data forms that provide information to and obtain parameters from the user. 3. put_title

Places the title window on the console at startup.

4. menu_setup

Defines the menu structure including those action functions called as the result of a menu item being selected.

5. fill_stat_wn

At startup, fills the status window with parameter values by repeated calls to the function stat_wind_wrt. stat_wind_wrt updates a specific line in the status window and is called any time a parameter changes.

6. Action functions

When a bottom-level menu option is selected, 1 of 14 action functions will be called. These functions will result in obtaining parameter(s) from the user, selecting an option, exiting the menu structure to display data, or terminating the algorithm.

7. check_parms

Checks all user-entered parameters before the algorithm is permitted to continue. If any invalid parameters are located, the user is notified and returned to the menu structure.

8. error_handler

Provides a single place for all error messages to be processed. All error messages are output through a pop-up window.

9. rec_sel_rec1

This function is used to extract records from the data set when the starting record number is greater than the final record. See the Figure 18 flowchart. A data structure containing the offset and record length of the selected records is returned to the calling procedure. Only the DP file is required to determine which records to use.

With the standard data orientation, this function begins with the final specified record in order to move forward through the buffer.

10. rec_sel_rec2

This function is similar to rec_sel_recl except for two differences: rec_sel_rec2 is used only when the starting record number is less than the final record, and it begins determining selected records with the start record. See Figure 19.

rec_sel_rec1 and rec_sel_rec2 were implemented as two separate functions to simplify the logic.

11.depth_sel_rec

This function is considerably different from the two rec_sel_rec? functions described above, even though its task is also to select records for display. It is used when the beginning and ending depths 


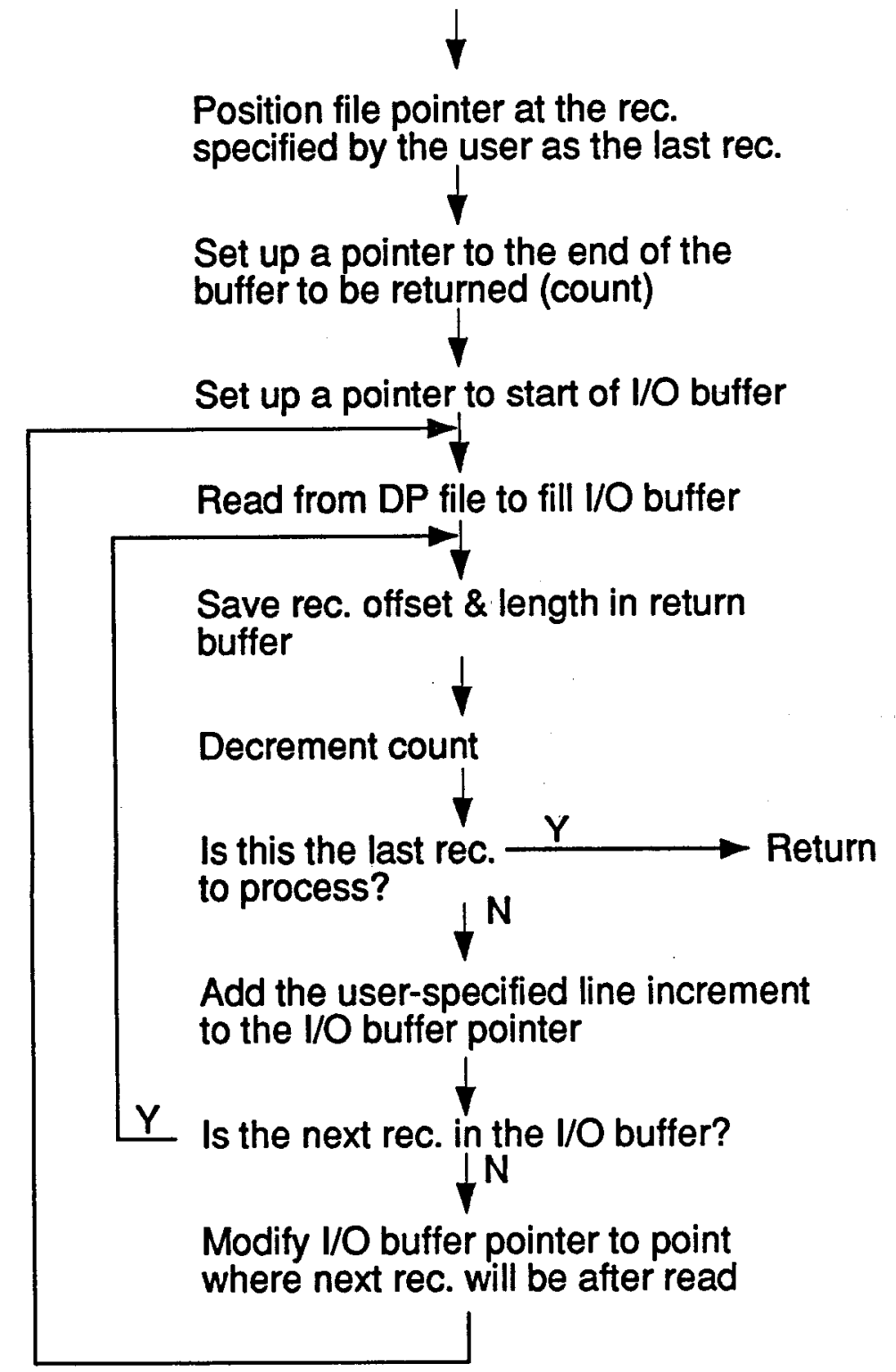

Figure 18. rec_sel_rec1 flowchart. 


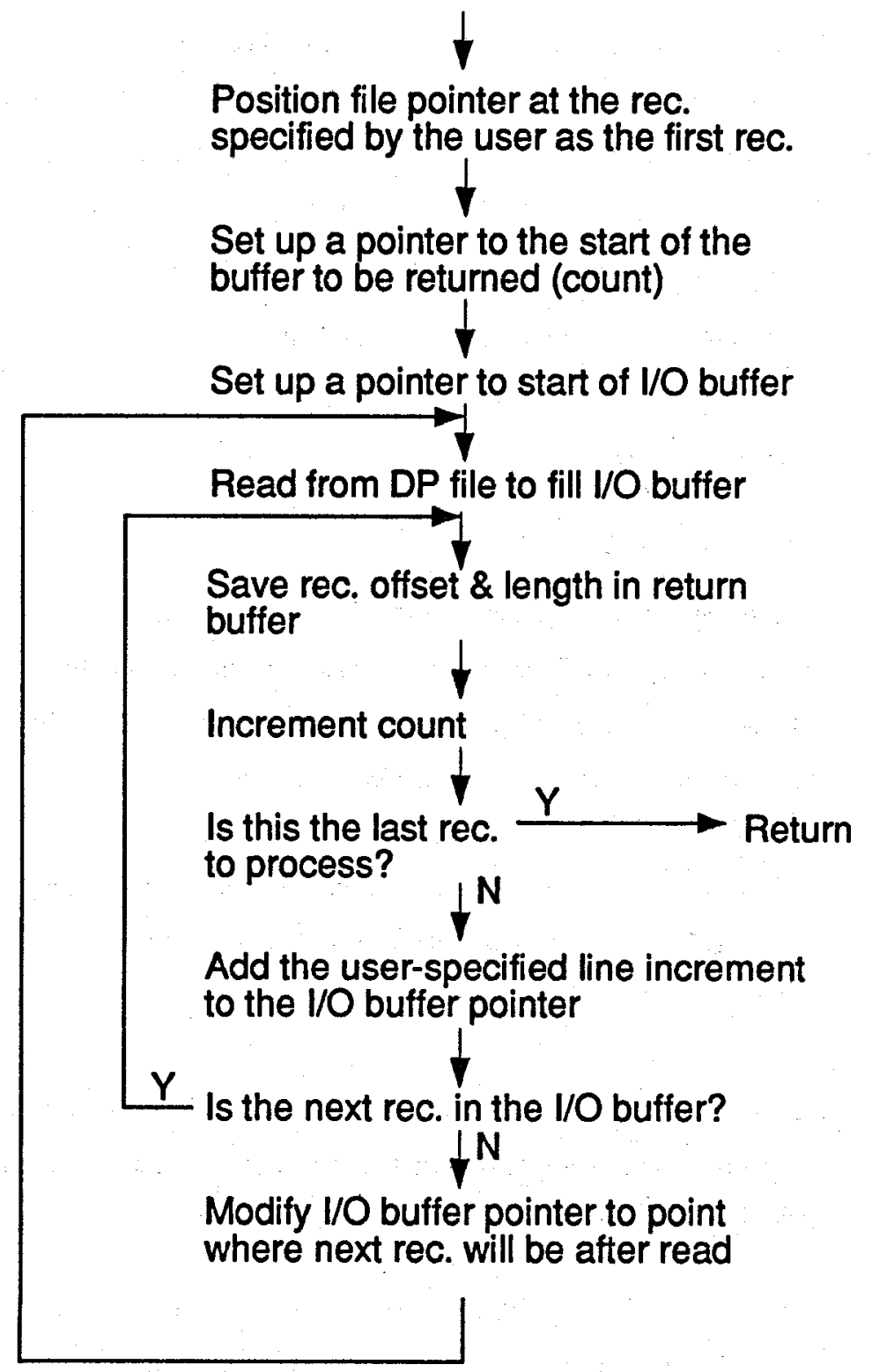

Figure 19. rec_sel_rec2 flowchart. 
are known and the actual record numbers are unknown.' Therefore, this function has to determine which records most closely match a set of target depths. See the Figure 20 flowchart. The function starts with the last specified depth as the first target depth. Three differences are calculated for each record processed:

$$
\begin{aligned}
& \text { prev_dif = ldepth of previous record }- \text { target depthl } \\
& \text { cur_dif = ldepth of current record }- \text { target depthl } \\
& \text { next_dif = ldepth of next record }- \text { target depthl }
\end{aligned}
$$

The following condition must be met:

$$
\text { prev_dif }>\text { cur_dif }<=\text { next_dif }
$$

for the current record to become a selected record. Once a record has been selected, the target depth is decremented by the user-specified increment value and processing continues.

Since the cur_dif becomes the prev_dif and next_dif becomes cur_dif for the next record, only the next_dif needs to be calculated for each record processed.

12. disp_recs

Using the list of selected records provided by one of the *_sel_rec functions, disp_recs reads and scales the data. The scaled data are then written to the specified location of a display channel. See Figure 21.

\section{3. recs_specs}

If records were selected on the basis of depth, this function tells the user which records were selected as the first and last displayed. The corresponding depths of the first and last records specified are displayed if in record mode.

\section{BATD User's Guide}

1. Menu Description. BATD is a menu-driven algorithm that provides a flexible user interface. Movement within menus is accomplished using the arrow keys. A menu item is selected by pointing to an item with the arrow keys followed by Enter or by pressing the first character in the menu item name. To move up one level in the menu, use the Escape key.

Four options make up the upper level menu. The Source group includes all the parameters used to specify data to be displayed. The actual display location is controlled by means of options in the Display group. Run will display the specified data. Exit returns the user to the operating system.

An overview of the menu structure is shown in Figure 22. Each of the options grouped under Source and Display will be discussed in the following segments:

\section{Source Filename}

Specify both the data and DP files from which data are to be displayed. 


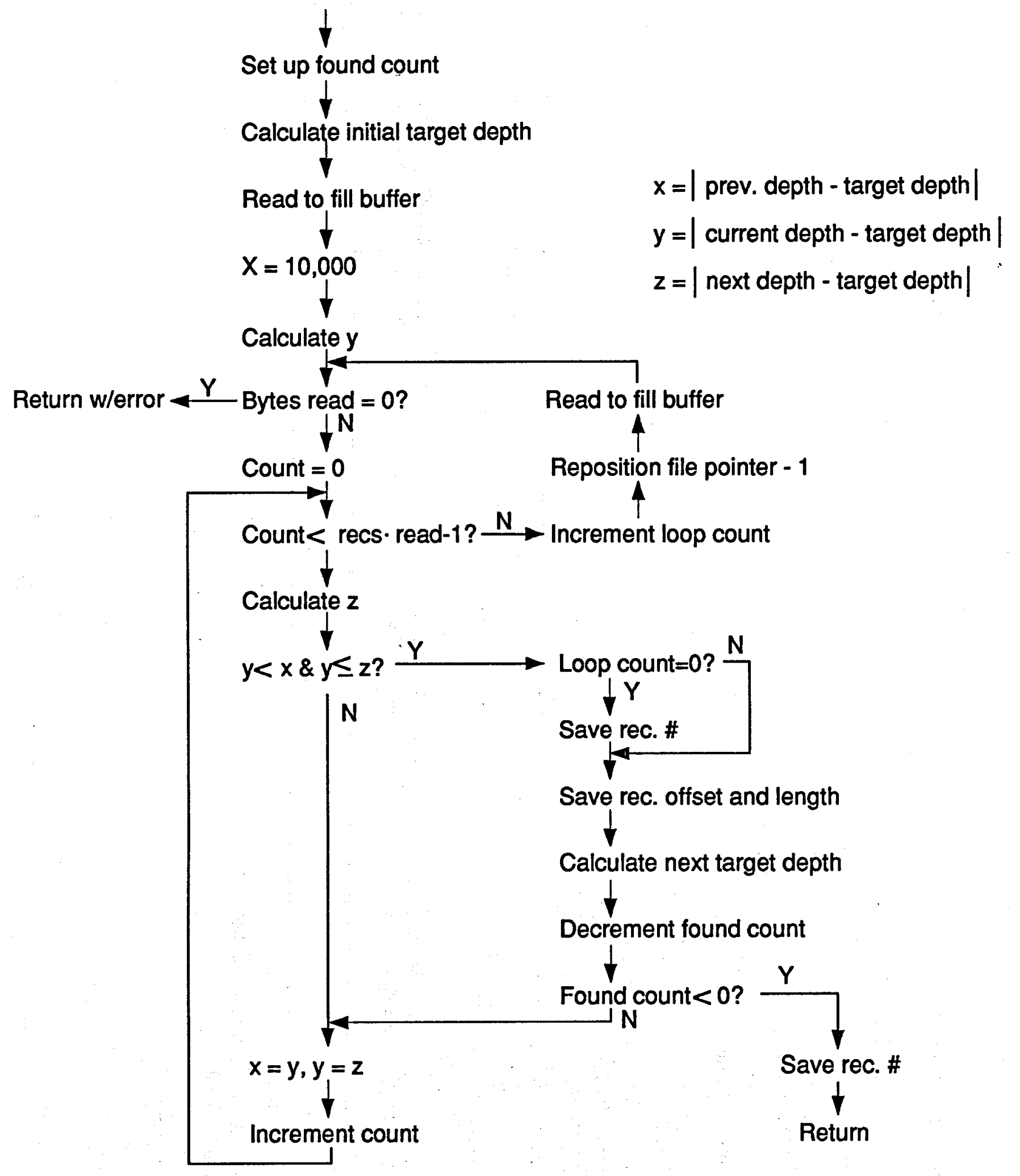

Figure 20. depth_sel_rec flowchart. 
Set up start display coordinates<smiles>[3H][V]</smiles>

Calculate scale values from scale

factors

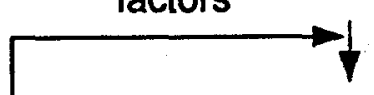

Last line to display ? $\stackrel{Y}{\longrightarrow}$ Return

$$
\downarrow \mathrm{N}
$$

Position file pointer on data file

\section{Read data}
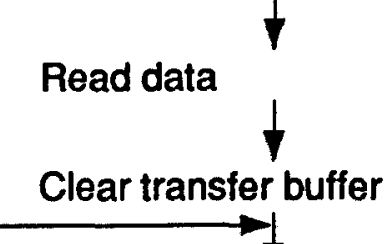

Last element in line? $\mathrm{Y}$

$\downarrow N$

Display amplitude data?

$$
\downarrow Y
$$

Transfer \& scale amplitude data from read buffer to transfer buffer

\section{Display travel tim}

Transfer \& scale travel time data from read buffer to transfer buffer
Transfer \& scale both types of data from read buffer to transfer buffer

Write contents of transfer buffer

to image memory

Figure 21. disp_recs flowchart. 


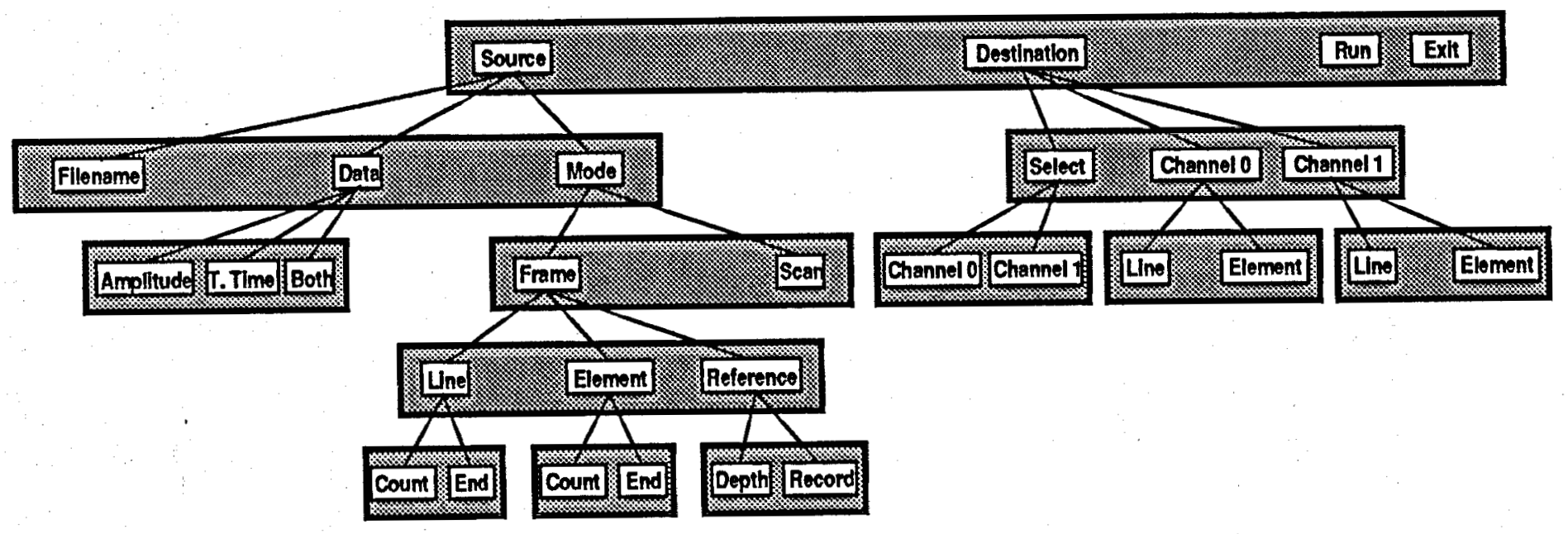

Figure 22. BATD menu structure. 
Source Data Amplitude

Display only the amplitude data (see Source Data Both).

Source Data T. time

Display only the travel time data (see Source Data Both).

Source Data Both

Display the amplitude data followed by the travel time data. A single column of intensity 0 will be inserted to separate the two data types.

Selecting any Source Data option will result in a prompt for a set of scale values. For Source Data Amplitude or Source Data T. time, only the appropriate scale value is needed. Scaling of data is described in the following equation:

$$
\text { display value }=\text { data value } / 2 \text { (scale value) }
$$

Scale values should be in the range of 0 to 7 inclusive.

Source Mode Frame Line Count

Source Mode Frame Line End

Data will be displayed as a single frame. A data form will ask which data records are to be displayed. These records may be specified by either a count or an end specification. The reference by which these records are specified is selected by Source Mode Frame Reference*. See Selecting Records Section below.

Note that the status window contains the first and last record numbers/depths contained in the file. This information is included to help generate the record specification. The record specification is checked extensively to make sure that the specified records are in fact contained within the data file.

Source Mode Frame Element Count

Source Mode Frame Element End

Identical to Source Mode Frame Line * except that elements from each data record are being selected.

Source Mode Frame Reference Depth

The records selected by Source Mode Frame Line * are specified by depth. A positive increment is required.

Source Mode Frame Reference Record

The records selected by Source Mode Frame Line * are specified by record number. The increment may be either positive or negative. See Logical Display Section below.

Source Mode Scan

The entire data file will be displayed by scrolling the display.

Scan mode is currently not implemented. 


\section{Destination Select Channel 0}

Destination Select Channel 1

When Run is executed, the specified data will be written to the selected display channel. These options also select which channel is displayed. By using these options, unique data can be written to both channels. Destination Channel 0 Line

Destination Channel 1 Line

Select the first display line to contain the first data record.

Display lines are numbered $0-479$ beginning at the top of the display.

\section{Destination Channel 0 Element}

Destination Channel 1 Element

Select the first display column to contain the first data element.

Display columns are numbered $0-511$ beginning at the left of the display.

When this menu structure is used, output similar to that shown in Figure 23 can be generated. Figure 24 contains the parameters used.

2. Selecting Records. Selecting records can be accomplished by either the count or the end methods. For either method, the first two parameters are a start and an increment value. The start value represents the first record to display. The increment value represents how many records to move from the first record to obtain the second and so on. For example, if the start value is 4 and the increment is 2 , records $4,6,8$,... would be selected.

The count or end specifications differ only in how the total number of records to select is determined. For count, the number of records is directly specified. In the end method, the approximate last record to select is specified by the user. The algorithm uses the start, increment, and end values to calculate the actual count. Then, using the start, increment, and calculated count, the algorithm determines the exact end value. The exact end value is used as the record specification and is written to the status window.

All values used in the record specification are limited to integer values.

3. Logical Display. As previously described, record selection may be made with respect to depth or record numbers. When depth reference is used, the display is limited to the logical format. In this format, the data from the shallowest portions of a wellbore are displayed at the top of the display, which provides a logical increase of depth as the eye travels down the display. For depth reference, the start depth must be smaller than the end depth and the increment must be positive.

The logical format restriction is removed when a record reference is used. This reference provides the user with additional freedom by supporting both positive and negative increments. Note that, when a positive increment is used in record reference, the data are not displayed in logical order. Only with a negative increment can this format be duplicated. 


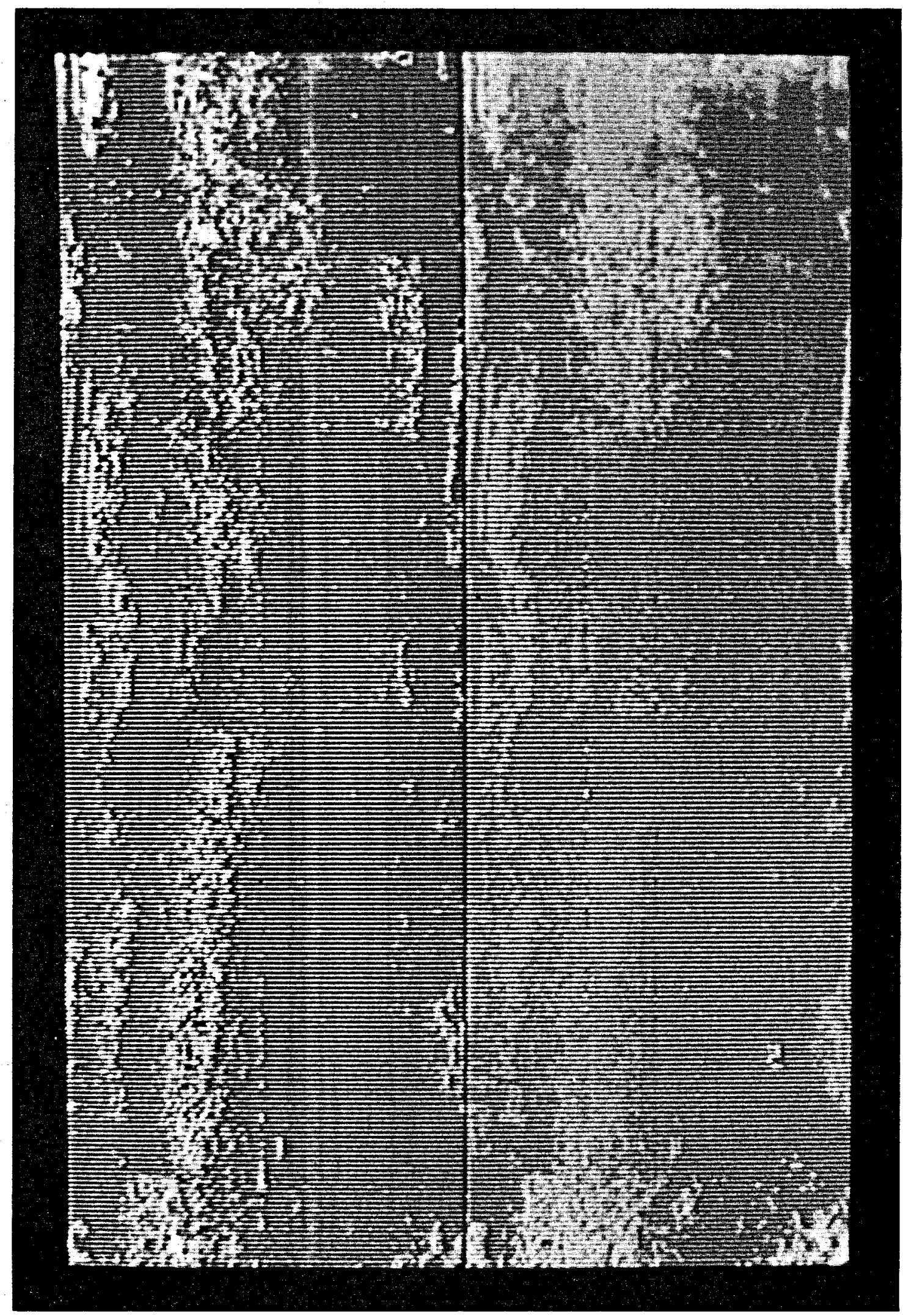

Figure 23. Example of display generated by BATD. 


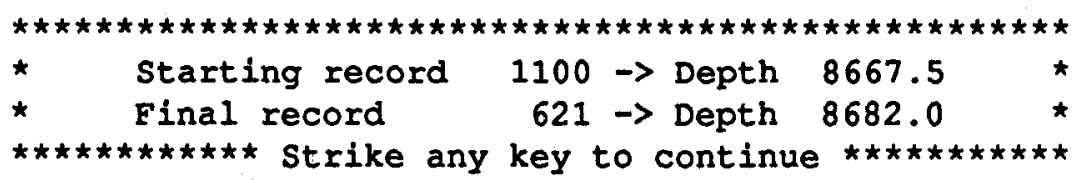

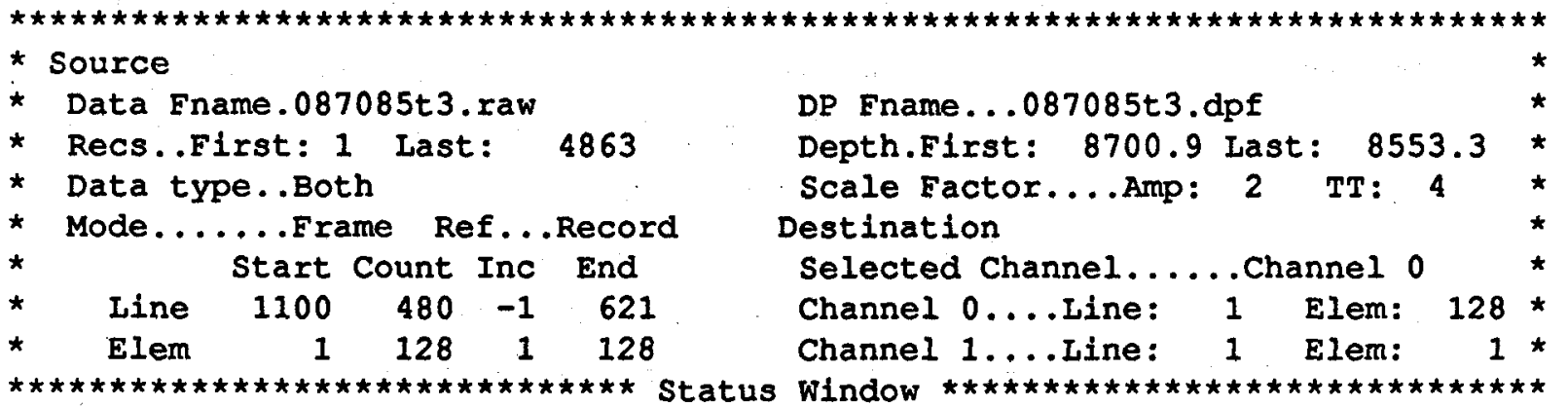

Figure 24. Parameters used to generate Figure 23. 


\section{BATT (BAT Trace Generation Utility)}

\section{A. Functional Description}

BATT is a utility designed to plot the travel time data as radial distance in inches vs sensor rotational position. Each plot generated on the display, referred to as a trace, is from a single revolution. Records from which to generate traces are identified with reference to depth by the user. Lines of reference, referred to as tick lines, and scaling parameters are specified by the user. The minimum and maximum radii found in the records in the interval bounded by two successive trace records are added to the display. Each trace is annotated with depth and tick line reference information. A video copier can easily generate hardcopies.

This utility is menu driven with pull-down submenus to provide the user with a friendly interface. Data forms are used to obtain parameters from the user. A status window is constantly updated to provide the user with current parameters. Windows relay additional information or error messages to the user.

\section{B. Implementation}

BATT is a complex algorithm simplified by the extensive use of functions. The main routine is predominantly a collection of function calls, which break the complex algorithm up into more understandable, smaller tasks. Figure 25 provides an overview of the algorithm.

Two special libraries of functions were used to help develop this code. The first, Windows for Data, Vermont Creative Software, was used to design/implement the menu structure. This library was also used to provide informational windows as well as data forms for entering data. Access to the image processing boards was provided by the DT-IRIS library from Data Translation.

To avoid passing numerous parameters between functions, global variables are used extensively. Information pertaining to these variables as well as to all the \#defines is contained in a set of include files. See Appendix B.

In developing BATT, it was assumed that the typical user would, upon selecting the records to use, spend most of his/her energies on fine tuning the display parameters. Since it takes much longer to identify the records and generate the interval calculations, all parameters selected or changed are watched closely. Only when the user changes a record selection parameter is the entire algorithm executed. This results in much faster execution when the user is simply adjusting display parameters.

To provide insight into the concepts involved in the implementation of this algorithm, each of the functions developed for BATT will be discussed in the following pages. Flowcharts are provided for the more involved functions.

1. init_parms

Initializes many of the global parameters to default values. Also sets up the column indices of the display coordinates for the tick lines and traces. 


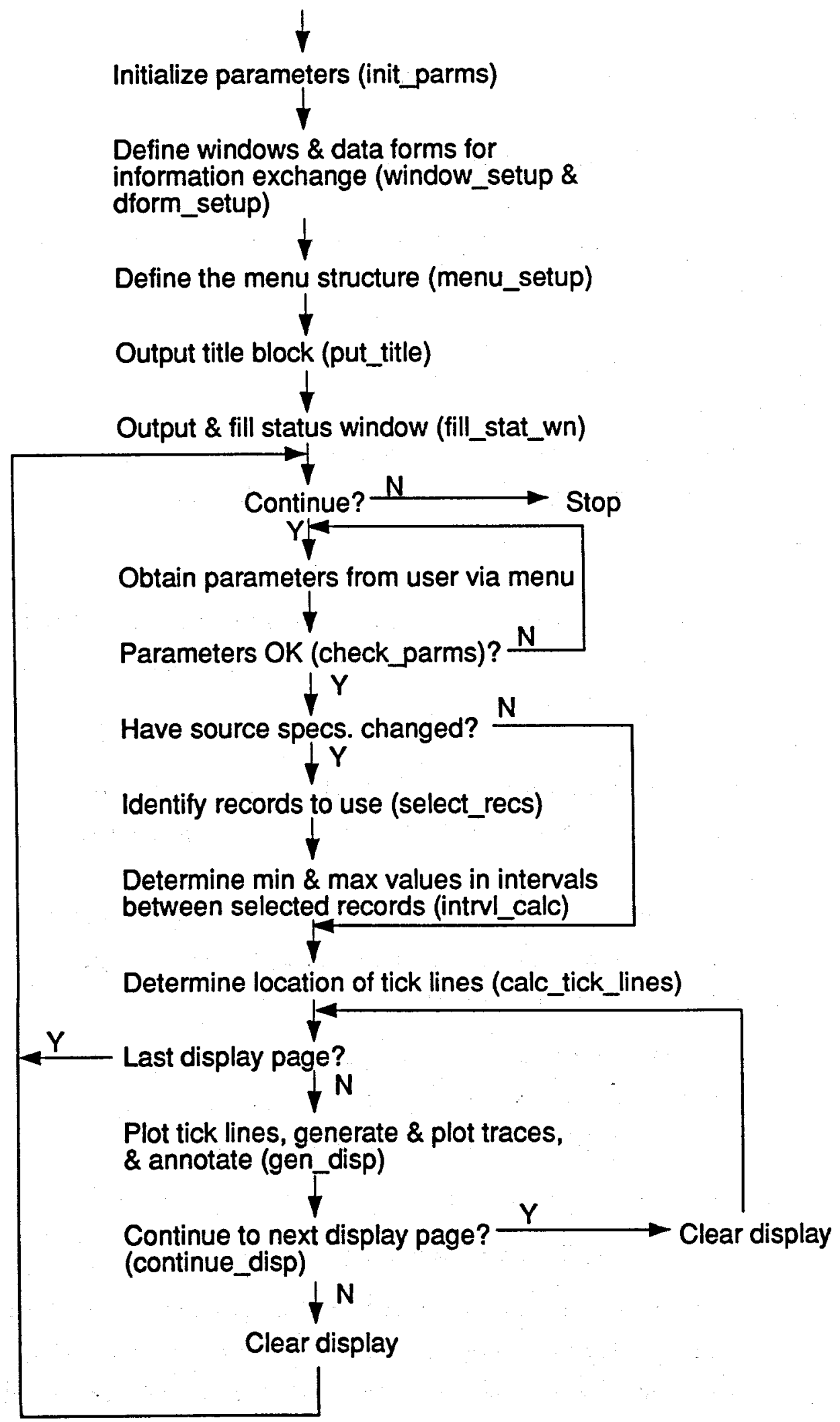

Figure 25. BATT flowchart. 
2. window_setup

Defines the windows used to provide information to the user.

3. dform_setup

Defines the data forms used to obtain parameters from the user.

4. menu_setup

Defines the menu structure including those action functions called as the result of a menu item being selected.

5. put_title

Places the title window on the console at startup.

6. fill_stat_wind

At startup, fills the status window with parameter values by repeated calls to the function stat_wind_wrt. stat_wind_wrt updates a specific line in the status window and is called any time a parameter changes.

7. Action functions

When a bottom-level menu option is selected, one of five action functions will be called. These functions will result in obtaining parameter(s) from the user, exiting the menu structure to generate traces, or exiting the algorithm.

8. check_parms

Checks all user-entered parameters before the algorithm is permitted to continue. If any invalid parameters are located, the user is notified and returned to the menu structure.

9. select_recs

This function determines which records most closely match a set of target depths. See the Figure 26 flowchart. The function starts with the last specified depth as the first target depth. Three differences are calculated for each record processed:

$$
\begin{aligned}
& \text { prev_dif }=\text { ldepth of previous record }- \text { target depthl } \\
& \text { cur_dif }=\text { ldepth of current record }- \text { target depthl } \\
& \text { next_dif = Idepth of next record - target depthl }
\end{aligned}
$$

The following condition must be met:

$$
\text { prev_dif }>\text { cur_dif }<=\text { next_dif }
$$

for the current record to become a selected record. Once a record has been selected, the target depth is decremented by the user-specified increment value and processing continues.

Since the cur_dif becomes the prev_dif and next_dif becomes cur_dif for the next record, only the next_dif needs to be calculated for each record processed. 


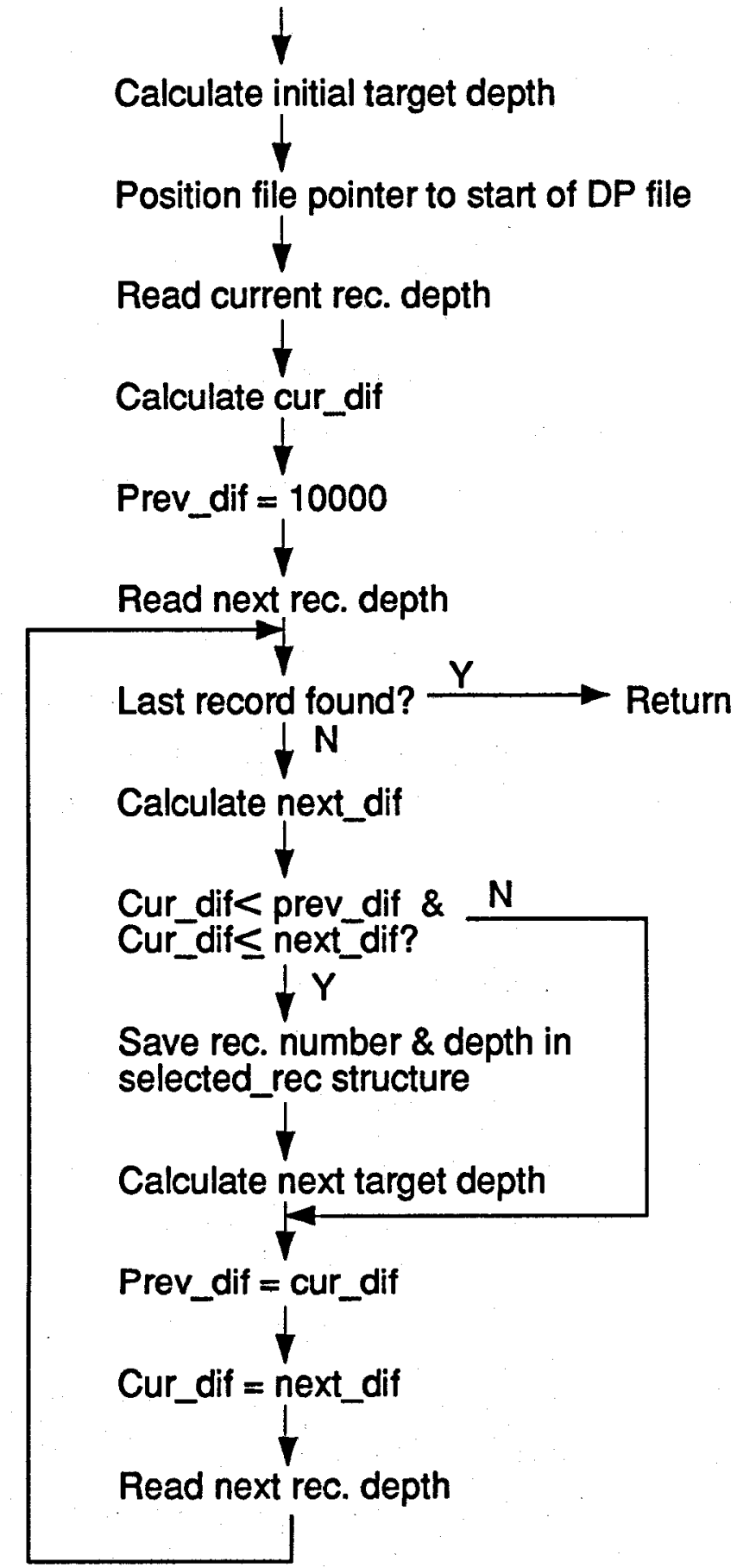

Figure 26. select_recs flowchart 
select_recs collects the depth and record number of each record identified in the selection process outlined above. This information is placed in the data structure named selected_recs for use by subsequent functions.

10. intrvl_calc

Determines the minimum and maximum radii found in the interval bounded by successive records identified by select_recs. The two bounding traces are not included in this calculation.

The resulting minimum and maximum are added to the selected_rec data structure partially filled in by select_recs. For a given interval, the minimum and maximum values are placed with the shallower bounding selected record. The final selected record therefore has no valid minimum and maximum values. See Figure 27.

11. calc_tick_lines

Calculates where the reference tick lines should be drawn. Since the starting and ending column are fixed by the number of travel time data points per record, only the line number is actually calculated. The number of traces per display page determines tick line location. See the Figure 28 flowchart. The results of this function are critical as gen_disp generates the traces with respect to the tick lines.

12. gen_disp

gen_disp is a busy function that handles three tasks:
a) draws the tick lines,
b) maps and draws the actual traces, and
c) adds all alphanumeric annotation.

The selected records, as specified in data structure selected_recs, must be located and read. gen_disp translates the record number into an offset into the data file. The travel time is read into a buffer and processed a word at a time. Each data point is mapped into physical display coordinates in three logical steps:
a) convert from caliper in millimeters $X 10$ to radius in inches,
b) calculate the offset from a base tick line in display lines, and
c) identify the physical line number with respect to the base tick line.

An array of display coordinate pairs is generated from the record. Once all the data from a single revolution are processed, the trace is drawn. See Figure 29.

13. continue_disp

Determines if the user wants to continue displaying traces or return to the menu.

\section{BATT User's Guide}

1. Menu Description. BATT is a menu-driven algorithm that provides a flexible user interface. Movement within menus is accomplished using the arrow keys. A menu item is selected by pointing to an item with the arrow 


\section{$\downarrow$}

Save 0 for min. \& max. for last selected rec.

Calculate no. of intervals

1

1

Set up an index into the selected rec.

structure

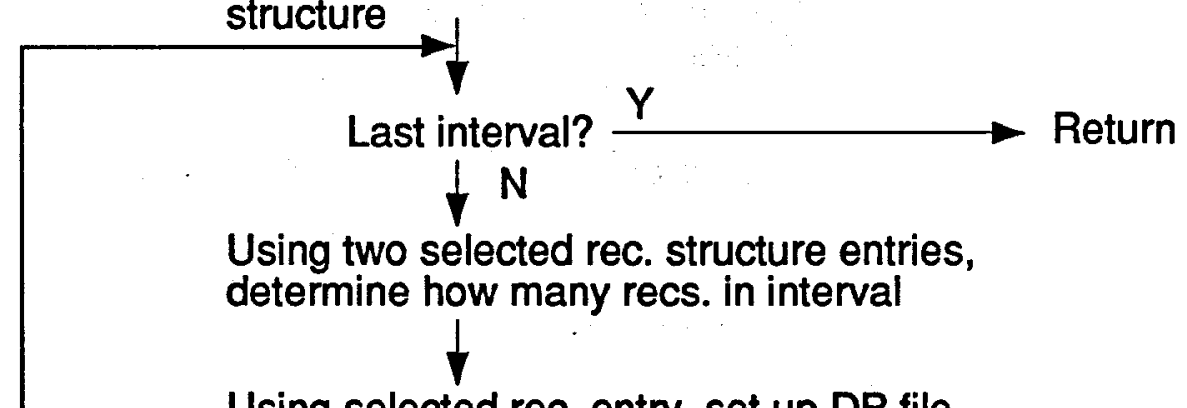

Using selected rec. entry, set up DP file pointer

Initialize min. \& max.

1

Last rec. in interval?

$\downarrow N$

Read a single entry from the DP file $\downarrow$

Set up the file pointer into data file<smiles>C[Al]1CC1</smiles>

Read only the travel time<smiles>[CH]1CCC1</smiles>

Update min. or max.

Save min. \& max. values in selected_rec structure

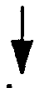

Decrement index into selected_rec structure

Figure 27. intrvl_calc flowchart. 


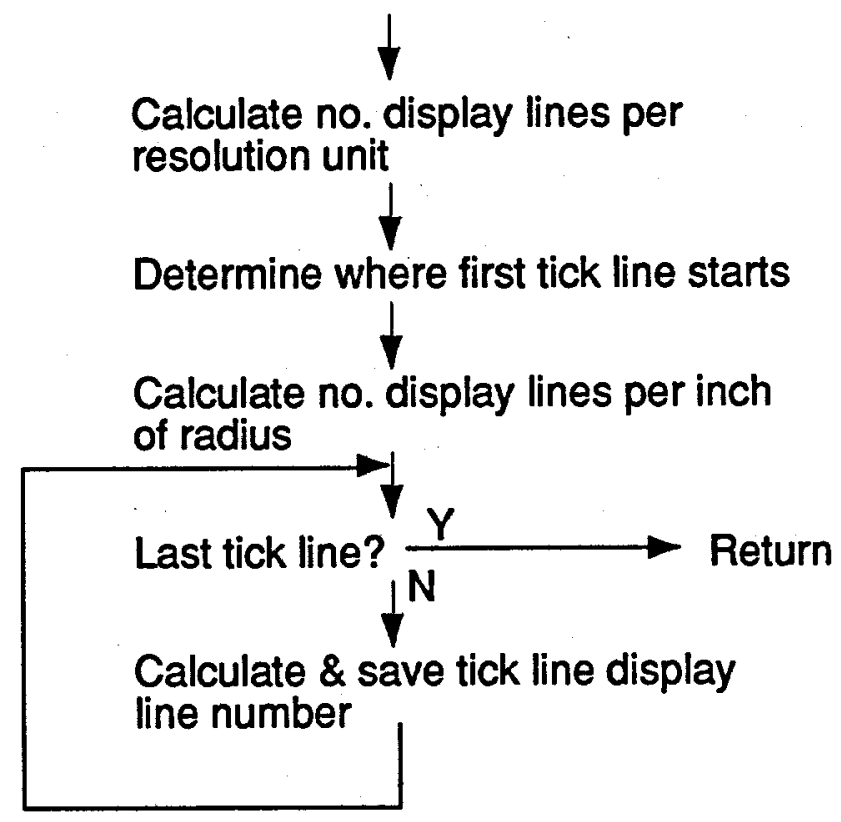

Figure 28. calc_tick_lines flowchart. 


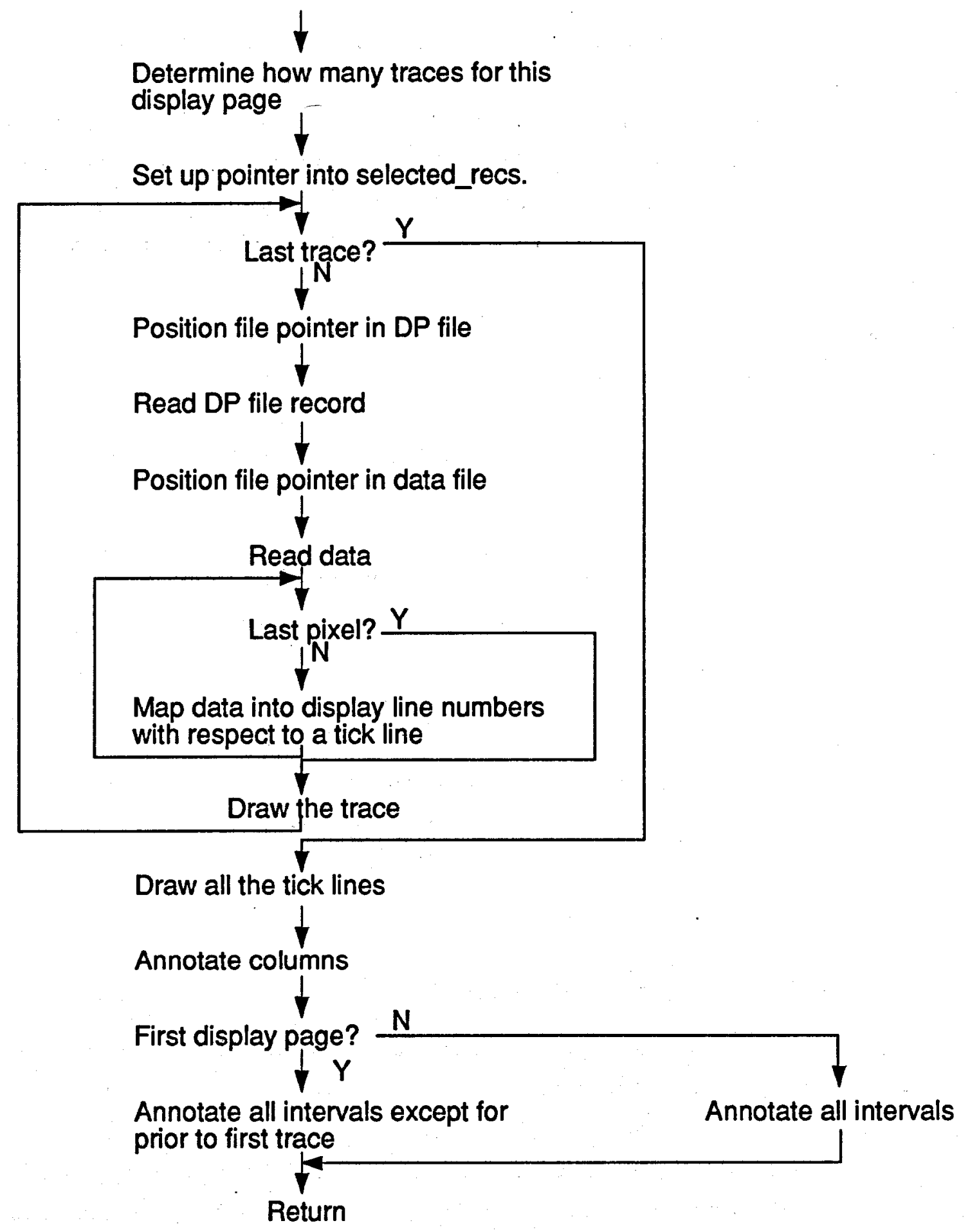

Figure 29. gen_disp flowchart. 
keys followed by Enter or by pressing the first character in the menu item name. To move up one level in the menu, use the Escape key.

Three options make up the upper level menu. The Parameters group includes all the parameters used to specify data from which traces are to be generated and the way in which those traces are to be displayed. Display will draw the specified traces. Exit returns the user to the operating system.

An overview of the menu structure is shown in Figure 30. Each of the options grouped under Parameters will be discussed in the following segments.

Parameters Filenames

Specify both the data and the DP files from which traces will be generated. Parameters Select Recs

Specify records with respect to depth from which to generate traces using the end method. See Section VII.C.2 for record specification details.

Parameters Display

User determines how the traces are to be placed on the display. The number of traces per display, base tick line value, and resolution between tick lines can be specified by this menu selection. See the next segment for definitions.

These menu items were used to generate the traces shown in Figure 31. Parameters used are included in Figure 32.

2. Display Definition. Several concepts need to be discussed in order to understand what the displayed traces represent and how the parameters under Parameters Display can be used. Consider the example shown in Figure 33. To generate this example, the following display parameters were used:

$\begin{array}{ll}\text { Traces per display: } & 2 \\ \text { Base tick line: } & 4.0 \mathrm{in} . \\ \text { Resolution between tick lines: } & 1.0 \mathrm{in} .\end{array}$

The number of traces per display controls the maximum number of traces that can be shown on a single display. If more traces have been specified by means of Parameters Select Recs, several successive displays, referred to as pages, are generated.

Each trace is generated with respect to a base tick line. The value in inches that the base tick line represents is specified as the base tick line value. In Figure 33, line b is the base tick line for Trace 1. Trace 2's base tick line is $\mathrm{c}$.

In addition to a base line, some unit of scale must be added to the display. The resolution-between-ticklines parameter controls the scale. This parameter specifies the physical distance represented on the display by the distance between two successive tick lines. This resolution applies to all the tick lines on the display. To illustrate this last point, Table I defines what each tick line represents to each trace. 


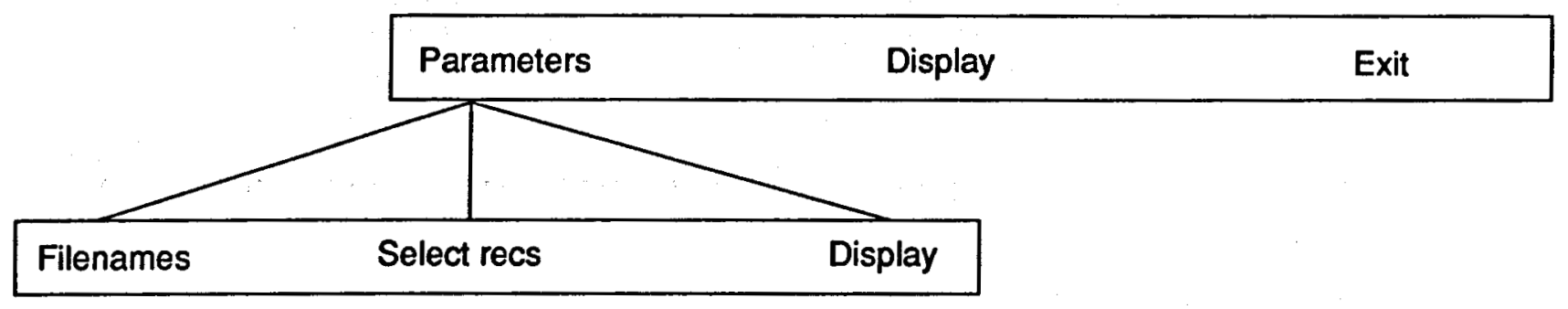

Figure 30. BATT menu structure.

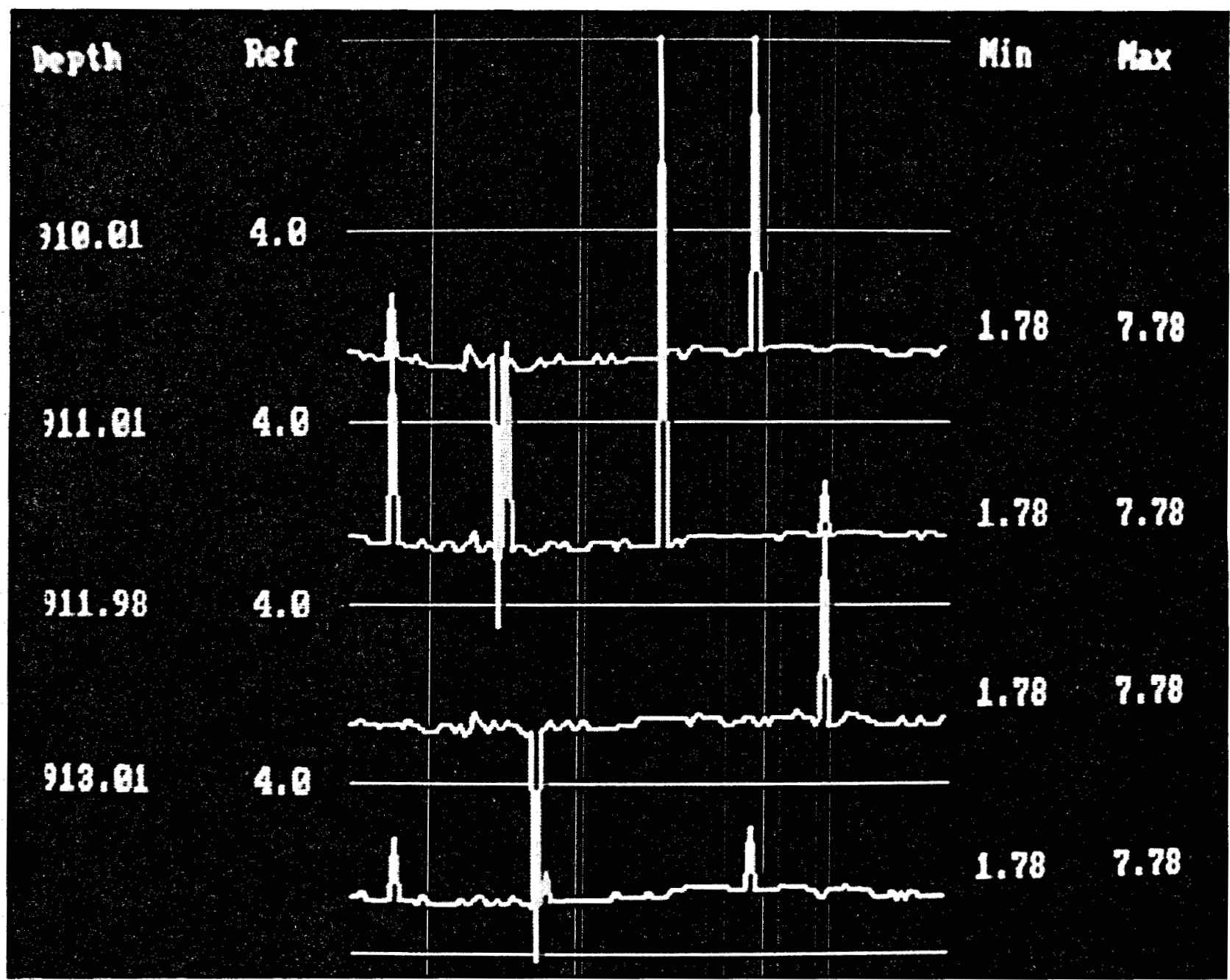

Figure 31. Typical traces generated by BATT. 


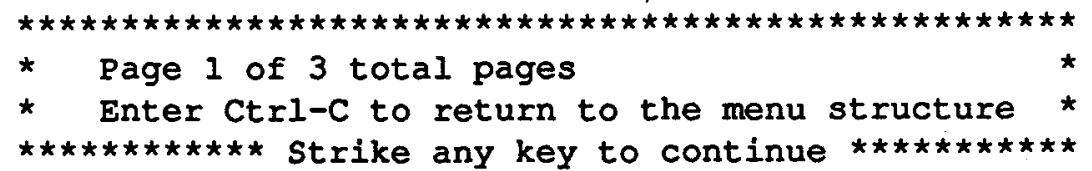

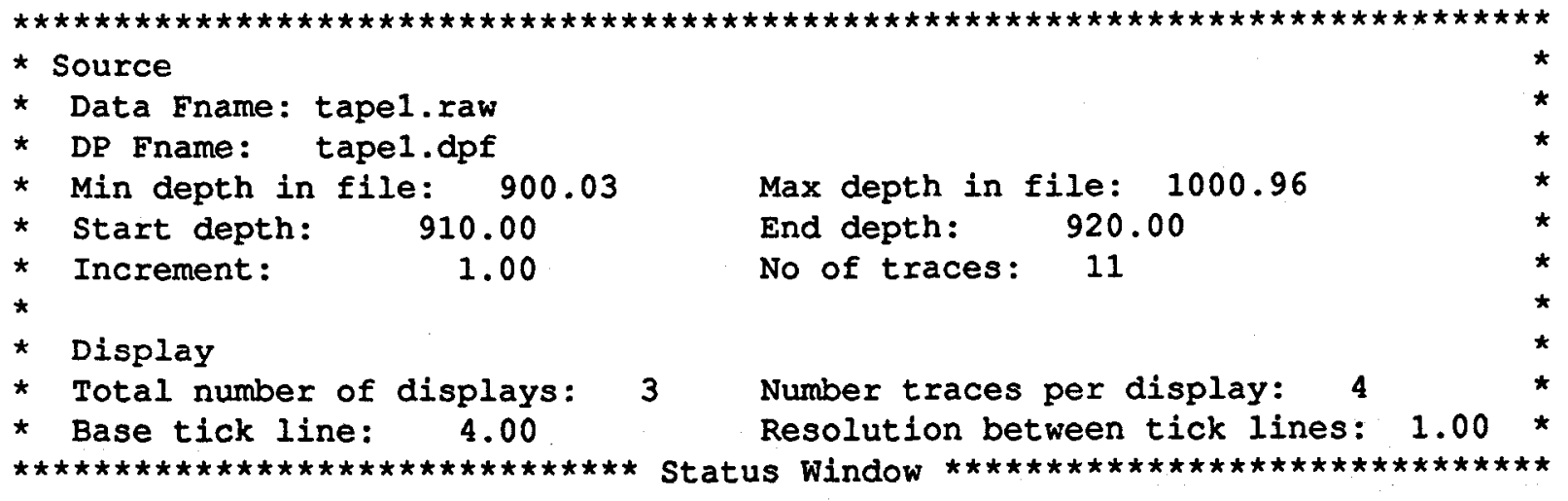

Figure 32. Parameters used to generate Figure 31. 


\section{Trace 1}

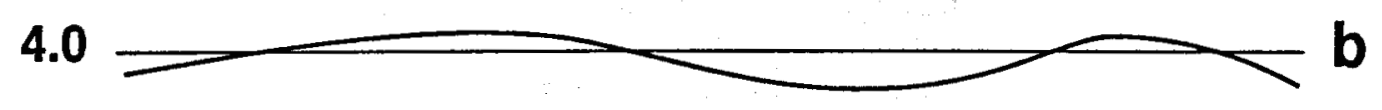

\section{Trace 2}

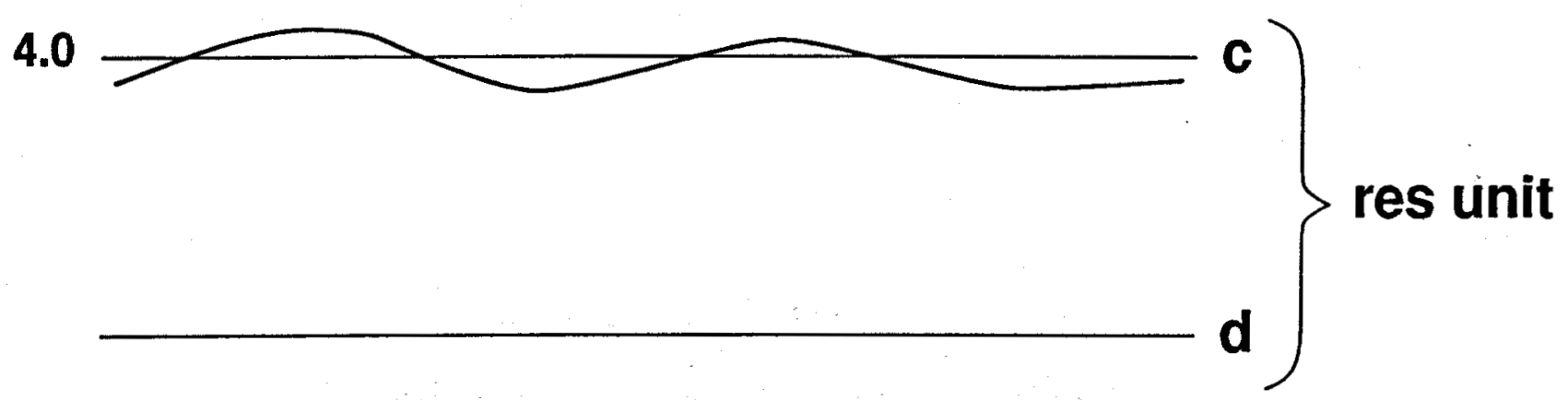

Figure 33. Example of two traces with associated tick lines. 


\section{TABLE I \\ TICK LINE REPRESENTATIONS}

\begin{tabular}{cc}
$\begin{array}{c}\text { With Respect } \\
\text { to Trace }\end{array}$ & $\begin{array}{c}\text { Tick } \\
\text { Line }\end{array}$ \\
\hline 1 & $\frac{\text { a }}{1}$ \\
1 & b \\
1 & c \\
1 & d \\
2 & \\
2 & a \\
2 & b \\
2 & c \\
& d
\end{tabular}

Notes:

\author{
Radius of \\ Base tick line +1 res unit $=4+1=5$ in. \\ Base tick line $=4$ in. \\ Base tick line -1 res unit $=4-1=3$ in. \\ Base tick line -2 res units $=4-2=2$ in. \\ Base tick line +2 res units $=4+2=6$ in. \\ Base tick line +1 res unit $=4+1=5$ in. \\ Base tick line $=4$ in. \\ Base tick line -1 res unit $=4-1=3$ in.
}
1) The base line for Trace 1 was tick line $b$, and for Trace 2 it was tick line $c$.
2) A specific tick line represents different radii for different traces on the same display.
3) By definition, each trace generated will always have at least three tick lines by which it can be referenced. They are a base tick line, base tick line +1 res unit, and base tick line - res unit.


3. Usage Notes. Much flexibility is provided in generating the trace display, but the user should be careful. For example, a base tick line value that is too big or too small coupled with a small value for the resolution between tick lines will produce traces that may be several resolution units away from the base tick lines. On the other hand, too large a resolution value will result in very little detail in the resulting trace.

The maximum number of traces per display that will still provide readable annotation is 32 .

\section{REFERENCES}

1. K. Hinz and R. Schepers, 1981, "SABIS--The Digital Version of the Borehole Televiewer," WBK, Institut fur Geophysik, Bochum, FDR.

2. T. K. Moore, K. Hinz, and J. Archuleta, "Development of a New Borehole Acoustic Televiewer for Geothermal Applications," 1985 International Symposium on Geothermal Energy, Vol. II (Geothermal Resources Council, Davis, California, 1985).

3. Vermont Creative Software, Windows for Data Reference Manual, Version 2.06.

4. Vermont Creative Software, Windows for C Reference Manual, Version 4.14.

5. Data Translation, Inc., DT-IRIS Reference Manual, Version 1.00. 

Appendix A - Workstation Development Environment

Hardware

IBM PC AT (6 MHz)

Core AT260 Disk Unit

Core ESDI Disk Controller

IBM 3.5-in. Disk Controller and External Drive Unit

AST Advantage Multifunction Board

IBM EGA Monitor

Video 7 Vega Deluxe EGA Adapter

Microsoft Bus Mouse

Maynard Maynstream Interface

Data Translation DT 2851 High Resolution Frame Grabber

Data Translation DT 2858 Auxiliary Frame Processor

Software

DOS 3.30 Operating System

Microsoft C Compiler, V4.0

Brief Programmers Editor, ${ }^{*}$ V2.0

Windows for $\mathrm{C}$, ${ }^{* *} \mathrm{~V} 4.14$

Windows for Data, ${ }^{* *}$ V2.06

DT-IRIS Subroutine Library, ${ }^{* * *}$ V1.01

* Solution Systems.

** Vermont Creative Software.

*** Data Translation. 


\begin{tabular}{|c|c|c|}
\hline$\frac{\text { Utility }}{\text { BDCOMP }}$ & $\begin{array}{l}\frac{\text { File }}{\text { CODEUMSCNATHBCOMP.C }} \\
\text { VOODEUMSCUBATBDCOMP. }\end{array}$ & $\begin{array}{l}\text { ContentslUse } \\
\text { All source code } \\
\text { Make file }\end{array}$ \\
\hline DATASEP & $\begin{array}{l}\text { VODEUMSCBATNDATASEP.C } \\
\text { VCODEMSC BATDATASEP. }\end{array}$ & $\begin{array}{l}\text { All source code } \\
\text { Make file }\end{array}$ \\
\hline DPDUMP & $\begin{array}{l}\text { VCODEMMSCBBATDPDUMP.C } \\
\text { VCODEUMSCBATDPDUMP. }\end{array}$ & $\begin{array}{l}\text { All source code } \\
\text { Make file }\end{array}$ \\
\hline BDINVRT & $\begin{array}{l}\text { VODEMMSCBATBDINVRT.C } \\
\text { VODEMMSCBAT BDINVRT. }\end{array}$ & $\begin{array}{l}\text { All source code } \\
\text { Make file }\end{array}$ \\
\hline BDEXTRCT & $\begin{array}{l}\text { VCODEMSCBAATBDEXTRCT.C } \\
\text { VCODEMMC BAT BDEXTRCT. }\end{array}$ & $\begin{array}{l}\text { All source code } \\
\text { Make file }\end{array}$ \\
\hline BATD & 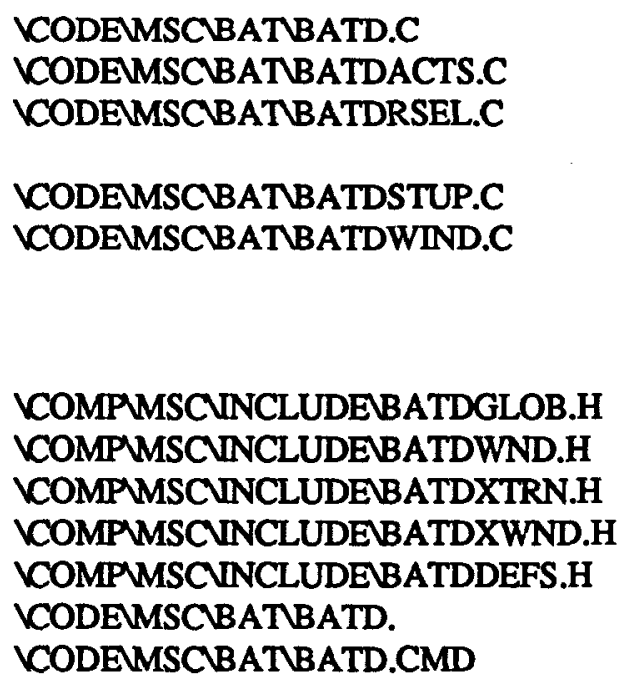 & $\begin{array}{l}\text { Main function only } \\
\text { All action functions } \\
\text { Functions depth_sel_rec,rec_sel_rec1, } \\
\text { rec_sel_rec2, and disp_recs } \\
\text { Functions init_parms and menu_setup } \\
\text { Functions window_setup put_title, } \\
\text { fill_stat_wn, stat_wind_wrt, } \\
\text { check_parms, error_handler, and } \\
\text { recs_specs } \\
\text { Global variable definitions } \\
\text { Global window/data form definitions } \\
\text { External variable declarations } \\
\text { External window/data form declarations } \\
\text { All \#defines } \\
\text { Make file } \\
\text { Link command line }\end{array}$ \\
\hline BATT & 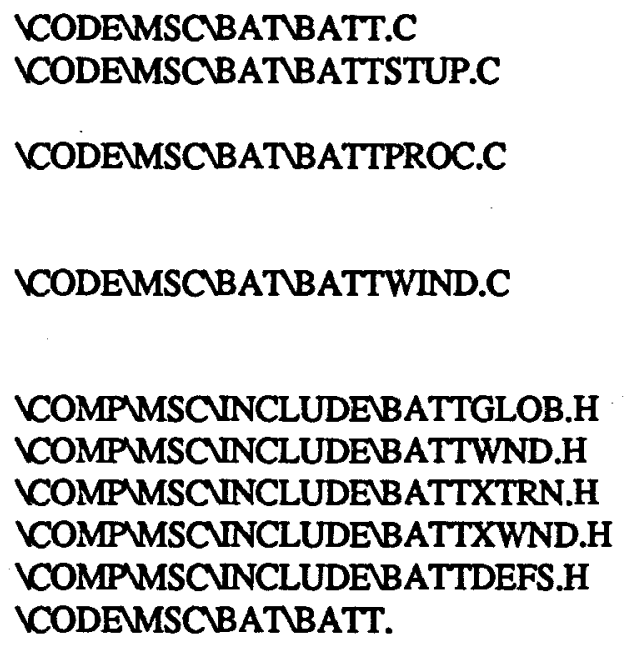 & $\begin{array}{l}\text { Main function only } \\
\text { Functions init_parms, window_setup, } \\
\text { dform_setup, and menu_setup } \\
\text { Functions check_parms, select_recs, } \\
\text { intrvl_calc, calc_tick_lines, gen_disp, } \\
\text { and doub_convrt } \\
\text { Functions put_title, fill_stat_wn, } \\
\text { stat_wind_wrt, all action functions, } \\
\text { error_handler, and continue_disp } \\
\text { Global variable definitions } \\
\text { Global window/data form definitions } \\
\text { External variable declarations } \\
\text { External window/data form declarations } \\
\text { All \#defines } \\
\text { Make file }\end{array}$ \\
\hline
\end{tabular}




\section{Appendix C - Field Tape Format}

Log Header Format*

\section{Byte \\ Offset}

$0-15$

$16-17$

$18 \cdot 23$

$24-27$

$28-29$

30

31

$32-47$

$48-63$

$64-79$

$80-95$

$96-101$

$102-105$

$106-109$

$110-111$

$112-115$

$116-121$

$122-125$

$126-127$

$128-131$

$132-135$

$136-139$

$140-171$

$172-187$

$188-219$

220 - 235

236 - 251

$252-267$

268 - 271

272 - 275

$276-279$

$280 \div 287$

288 - 295

296 - 303

$304-307$

$308-315$

316 - 323

$324-331$

$332-511$

512 - 1023
Length

16

2

6

4

2

1

1

16

16

16

16

6

4

4

2

4

6

4

2

4

4

4

32

16

32

16

16

16

4

4

4

\section{Field Description}

Identification string Sabis Field Tape Tape number

Date log began in YYMMDD format Time log began in HHMM format Experiment number Metric or English unit indicator Mud log available ( $\mathrm{Y}$ or $\mathrm{N}$ )

Well number/name Location/field name

Country

State

Tool name

Transducer frequency in $\mathrm{KHz}$

Shots per revolution

Revolutions per second

Tool software version

Control unit name

Control unit software version

Revolutions per revolution record

Number bytes in revolution header

Number amplitude data bytes

Number caliper data bytes

Logging company name

Logging engineer

Client company

Witness

Borehole fluid

Borehole fluid additives

Specific weight in $\mathrm{g} / \mathrm{l}$

Viscosity

Acoustic speed in $\mathrm{km} / \mathrm{s}$

Casing shoe depth in $\mathrm{mm}$

Fluid depth in $\mathrm{mm}$

Total depth in $\mathrm{mm}$

Drilled caliper in $\mathrm{mm}$

Unused, filled with 0

Additional comment text

* All information is ASCII. 


\section{Appendix C - Field Tape Format}

Revolution Record Format

\section{Byte}

Offset

$\begin{array}{rrr}0 & - & 7 \\ 8 & - & 15 \\ 16 & - & 21 \\ 22 & - & 25 \\ 26 & - & 27 \\ 28 & - & 29 \\ & & 30 \\ & & 31\end{array}$

$32-33$

$34-35$

$36-37$

$38-39$

$40-41$

$42-43$

$44-45$

46

47

$48-303$

$304-559$
Length

8

8

6.

4

2

2

1

1

2

2

2

2

2

2

2

1

1

256

256

\section{Field Description}

Identification string Sabislog

Collection depth

Collection time in HHMMSS format

Revolution counter

North indicator

Caliper offset

Fire voltage

Amplitude attenuation

Temperature \#1

Temperature \#2

Z-axis accelerometer minimum

$\mathrm{Z}$-axis accelerometer maximum

Y-axis tilt

$\mathrm{X}$-axis tilt

Flux gate intensity

Interest indicator

Unused

128 words of amplitude data

128 words of travel time data 


\section{Appendix D - Typical Terminal Session Output}

\section{$\mathrm{C}:>\mathrm{bdcomp}$}

BAT Tool Data Complement and Header Fix Algorithm

$\mathrm{v} 1.0$, Troy $\mathrm{K}$. Moore

Enter input filename: tape1.rtd

Enter output filename: tapel.ctd

Possible fix at end of file ignored

2 fixups were made to $\log$ header records

18 repositions were executed

2097152 bytes were processed

C:>datasep

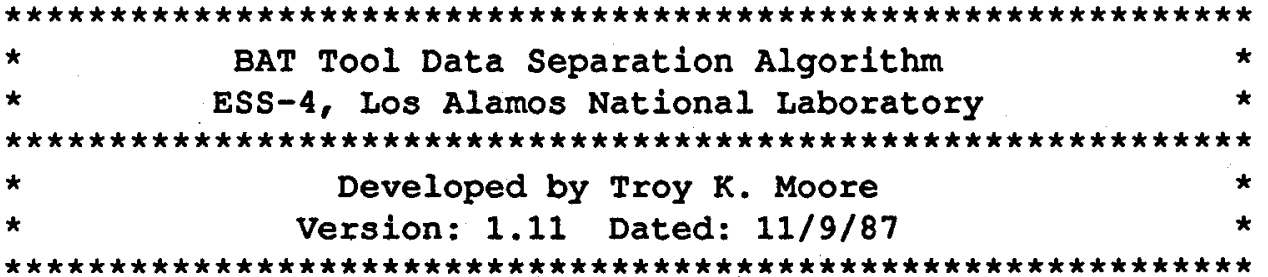

Enter input file name: tapel.ctd

Default output file names are as follows:

Data file: TAPE1.RAW

Data pointer file: TAPE1.DPF

Log header file: TAPE1.LHF

To accept these file names, hit the Enter key.

To generate alternate file names, hit any other key.

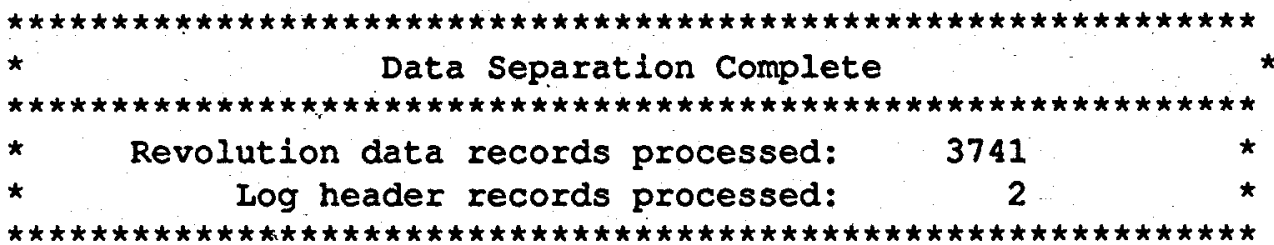




\section{Appendix D - Typical Terminal Session Output}

C:>dpdump tapel.dpf 56010

Data Pointer File Dump Utility, Version 1.0

File name: tape1.dpf

Flag: $560+/-10$

\begin{tabular}{|c|c|c|c|c|c|c|c|}
\hline $\begin{array}{l}\text { Record } \\
\text { Number }\end{array}$ & $\begin{array}{l}\text { Offset } \\
\text { in hex }\end{array}$ & Depth & $\begin{array}{l}\text { Record } \\
\text { Length }\end{array}$ & $\begin{array}{l}\text { I Record } \\
\text { I Number }\end{array}$ & $\begin{array}{l}\text { Offset } \\
\text { in hex }\end{array}$ & Depth & $\begin{array}{l}\text { Record } \\
\text { Length }\end{array}$ \\
\hline 1 & 0 & 1000.96 & 560 & 57 & $7 a 80$ & 1000.96 & 560 \\
\hline 2 & 230 & 1000.96 & 560 & 58 & $7 \mathrm{cbo}$ & 1000.96 & 560 \\
\hline 3 & 460 & 1000.96 & 560 & 59 & 7 ee 0 & 1000.96 & 560 \\
\hline 4 & 690 & 1000.96 & 560 & 60 & 8110 & 1000.96 & 560 \\
\hline . & . & . & - & . & - & - & - \\
\hline - & - & - & - & - & - & - & - \\
\hline - & - & - & - & - & - & - & - \\
\hline
\end{tabular}

C: >bdinvrt

BAT Data Inversion Utility

Developed by Troy K. Moore

version 1.00

<Input Filenames>

DP File: tape1.dpf

Data File: tapel.raw

<Output Filenames>

DP File: tapelin.dpf

Data File: tapelin.raw

3741 records were processed

C: $>$ bdextrct

BAT Data Extraction Utility

Developed by Troy $\mathrm{K}$. Moore

Version 1.00

<Input Filenames>

DP File: tapel.dpf

Data File: tapel.raw

<Output Filenames>

DP File: tapelex.dpf

Data File: tapelex.raw

<Record Specifications>

Start extraction with record: 45

Final record to extract: 90

50 records were extracted

54 


\section{Appendix E - BDCOMP Source Code}

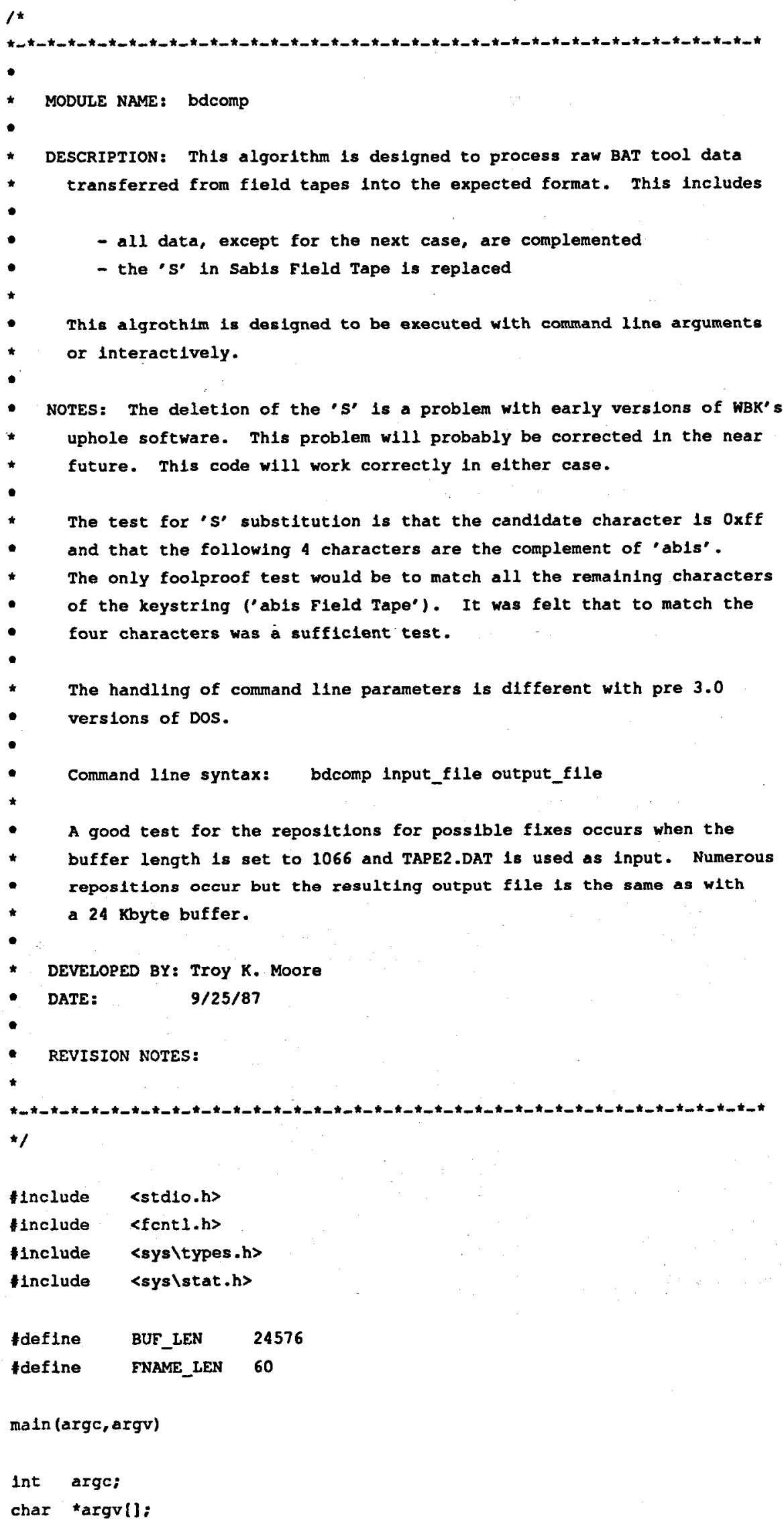




\section{Appendix E - BDCOMP Source Code}

1

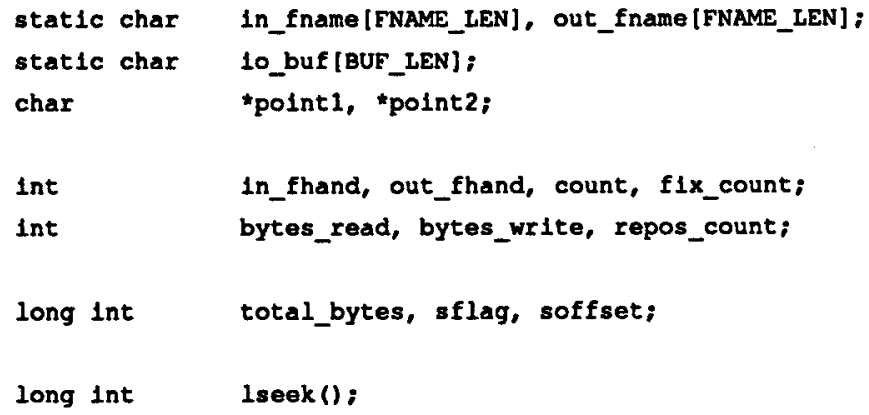




\section{Appendix E - BDCOMP Source Code}

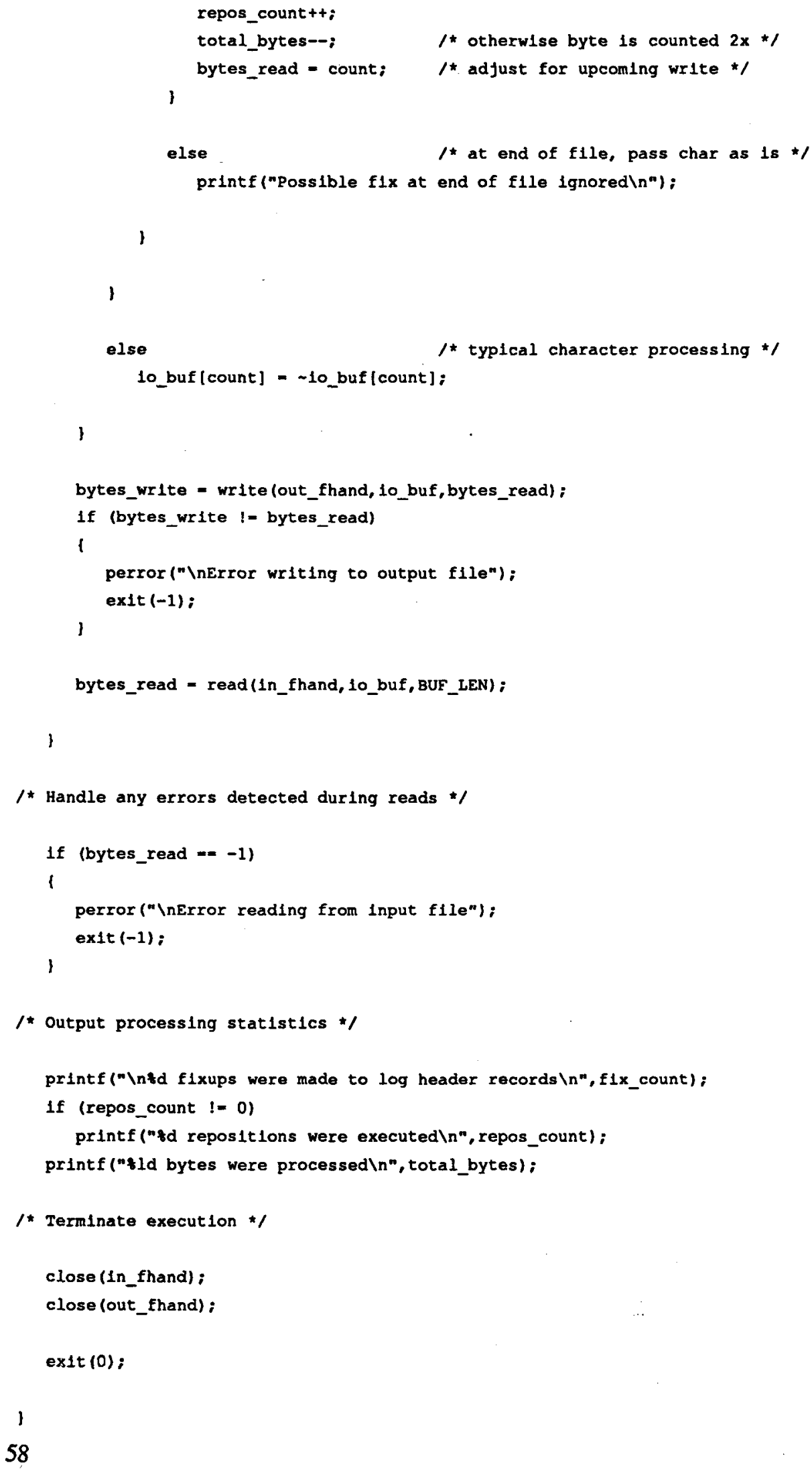




\section{Appendix F - DATASEP Source Code}

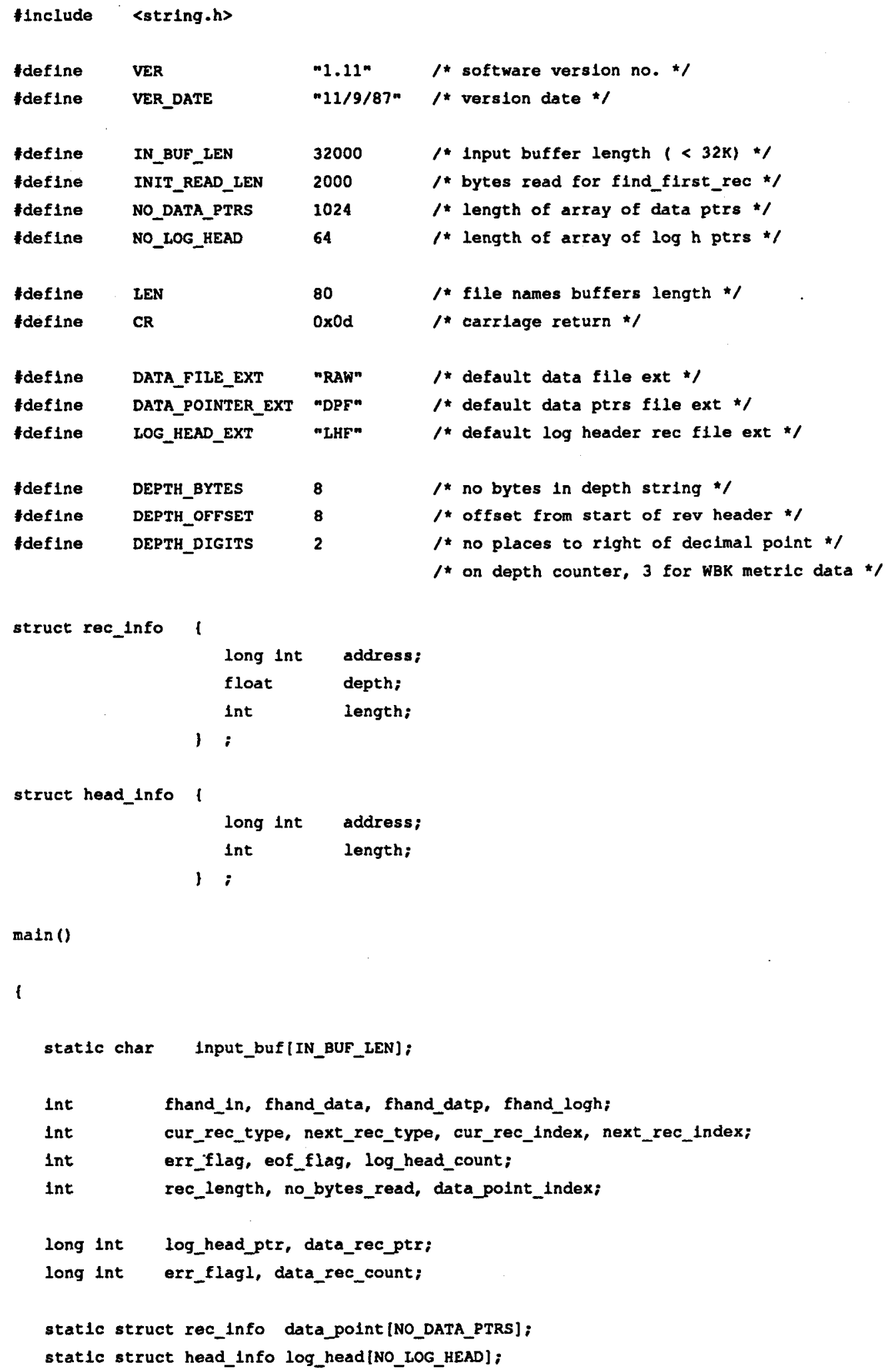




\section{Appendix F - DATASEP Source Code}

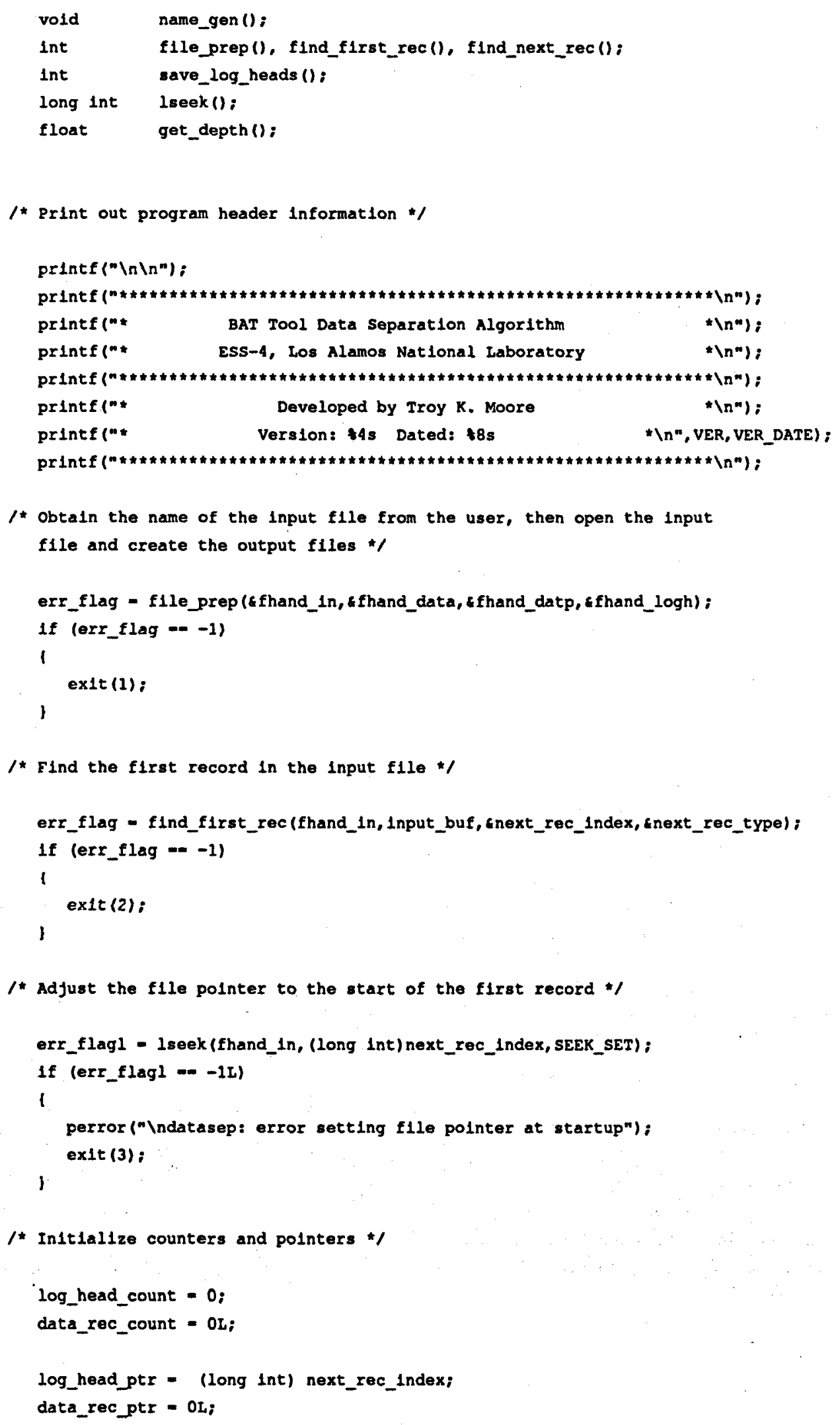




\section{Appendix F - DATASEP Source Code}

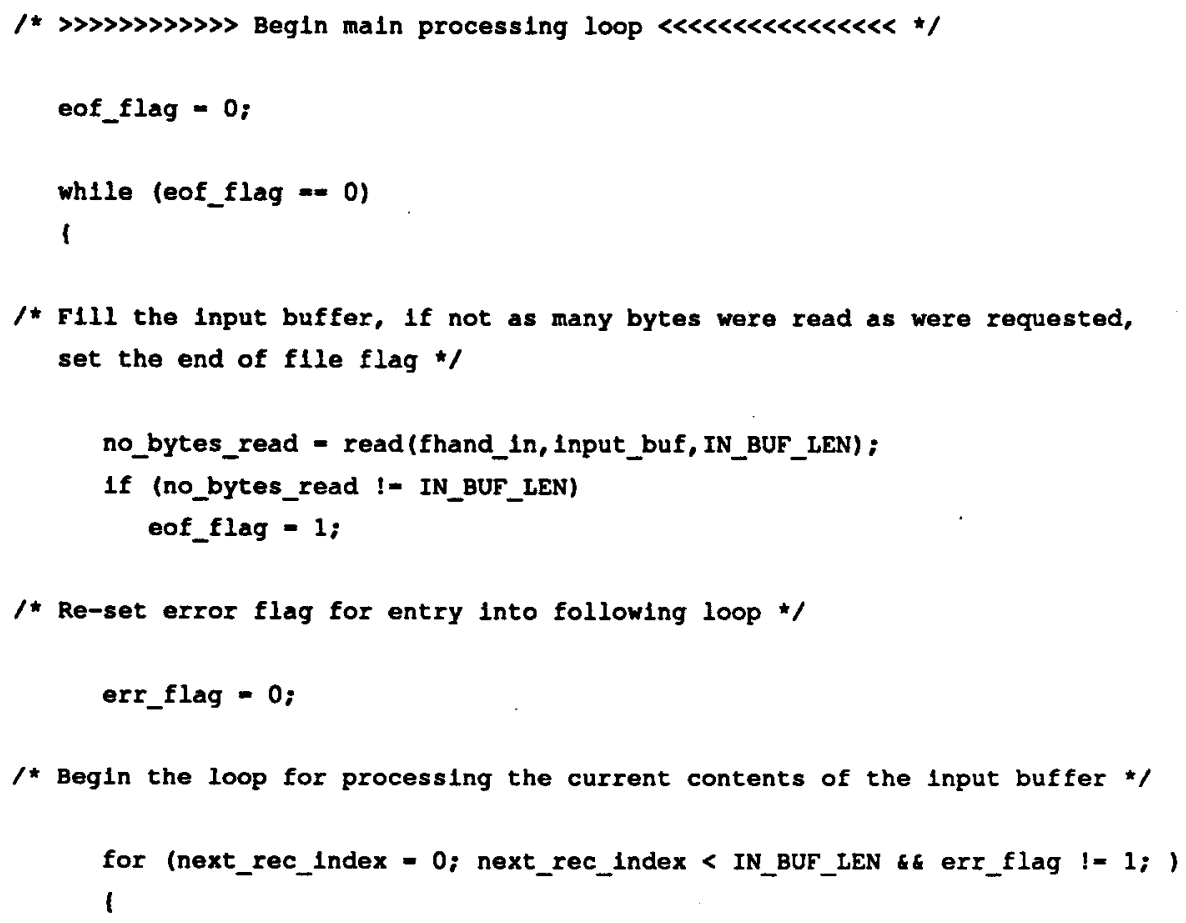




\section{Appendix F - DATASEP Source Code}

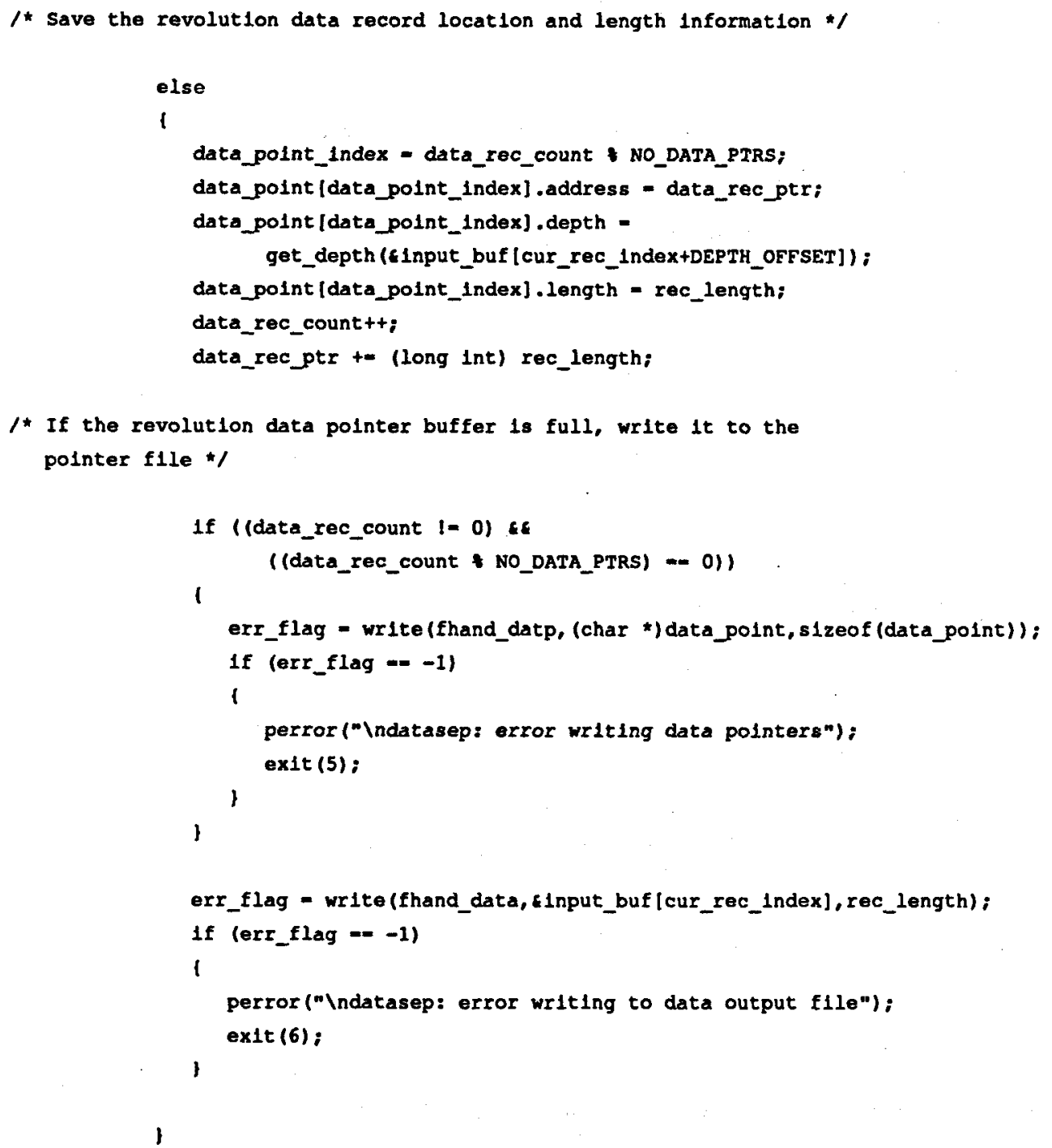




\section{Appendix F - DATASEP Source Code}

/* If end of buffer is detected (by find next_rec) but it is not the end of the flle, re-position the file pointer for the next read */

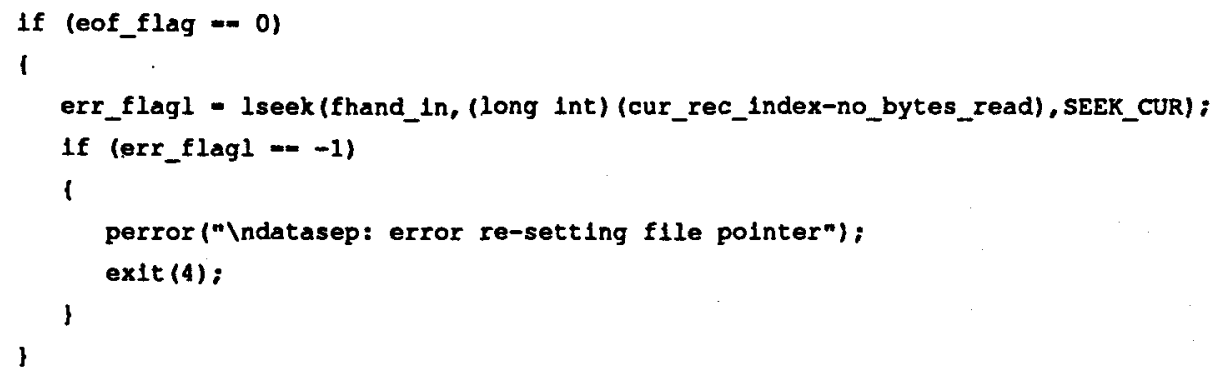




\section{Appendix F - DATASEP Source Code}

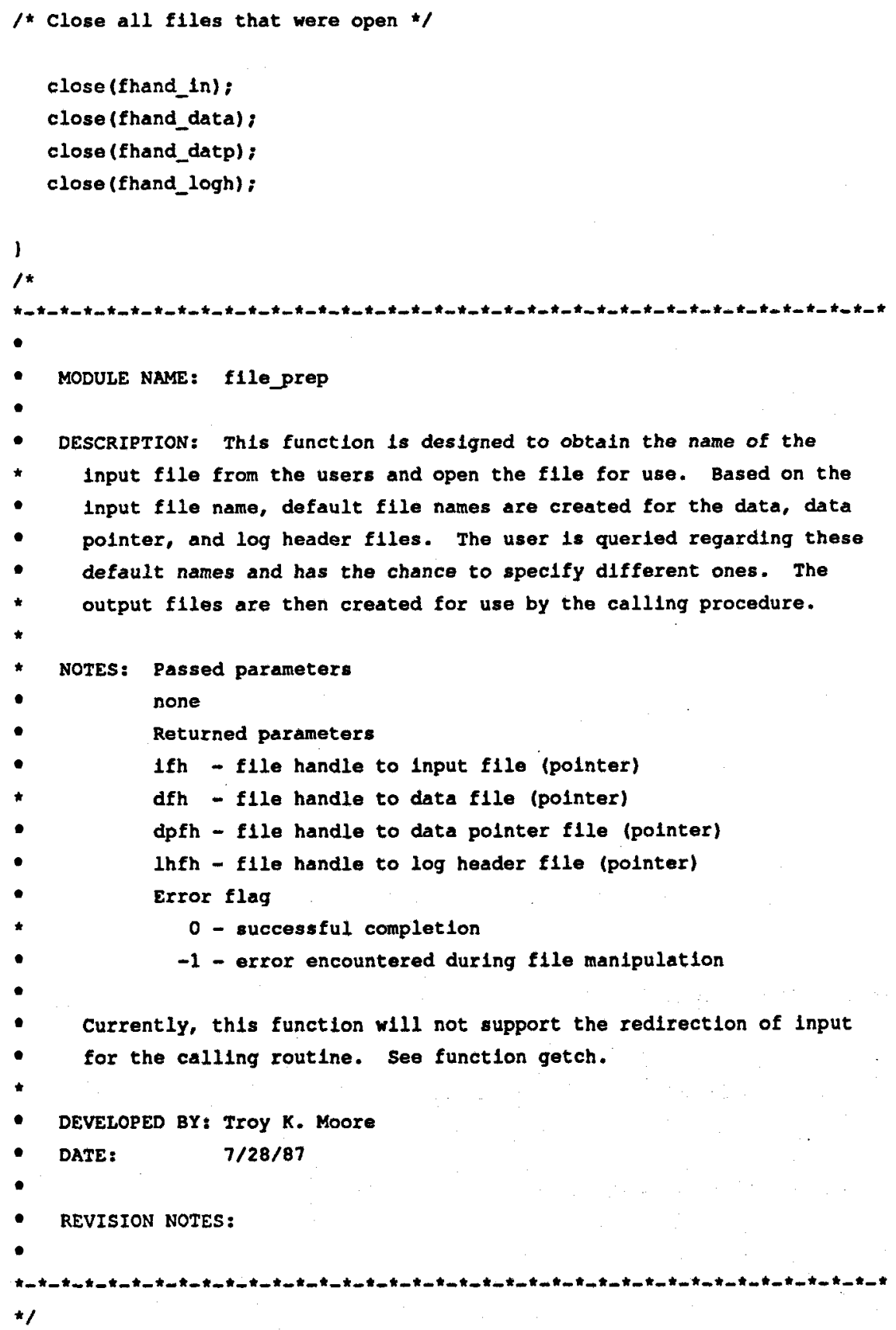




\section{Appendix F - DATASEP Source Code}

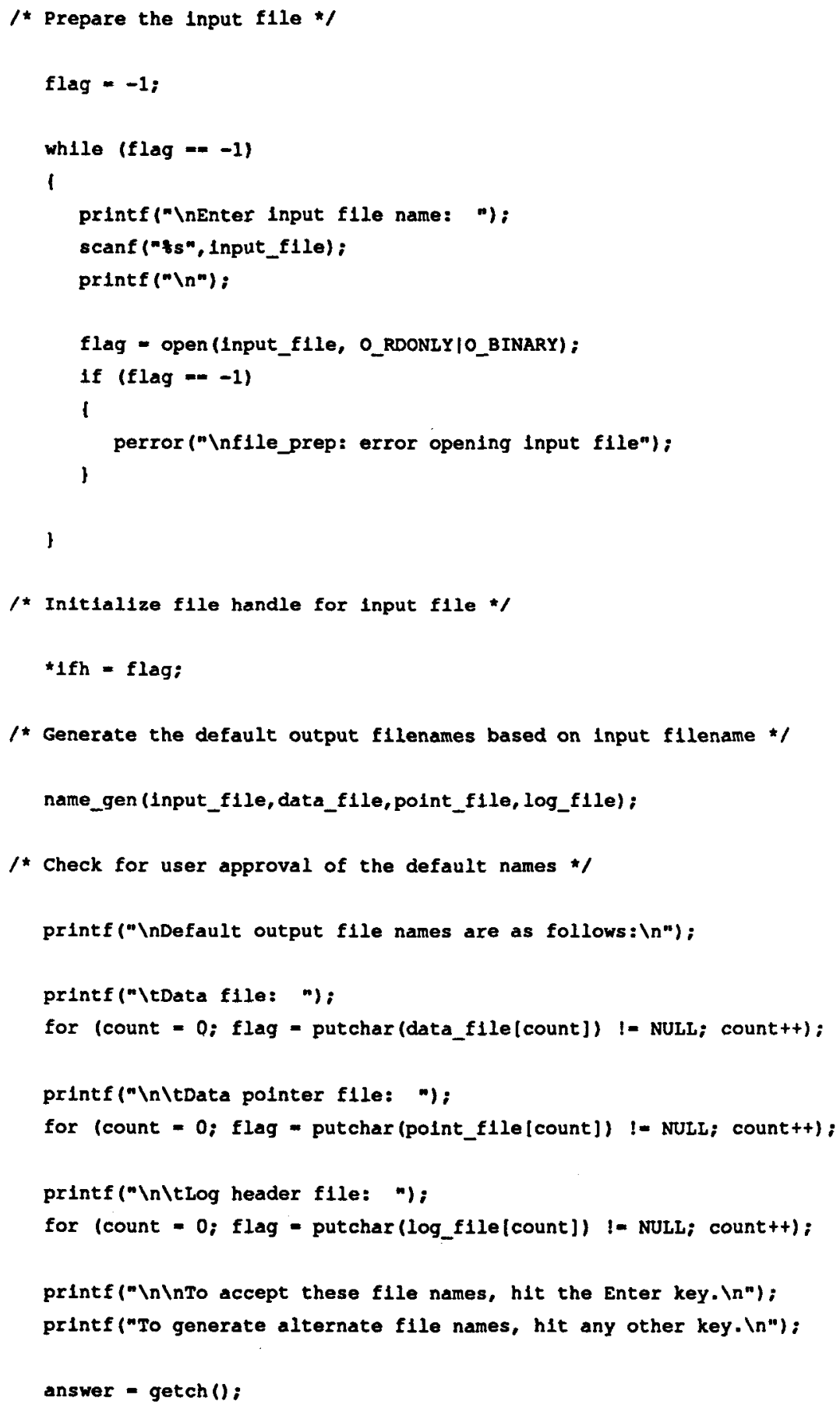




\section{Appendix F - DATASEP Source Code}

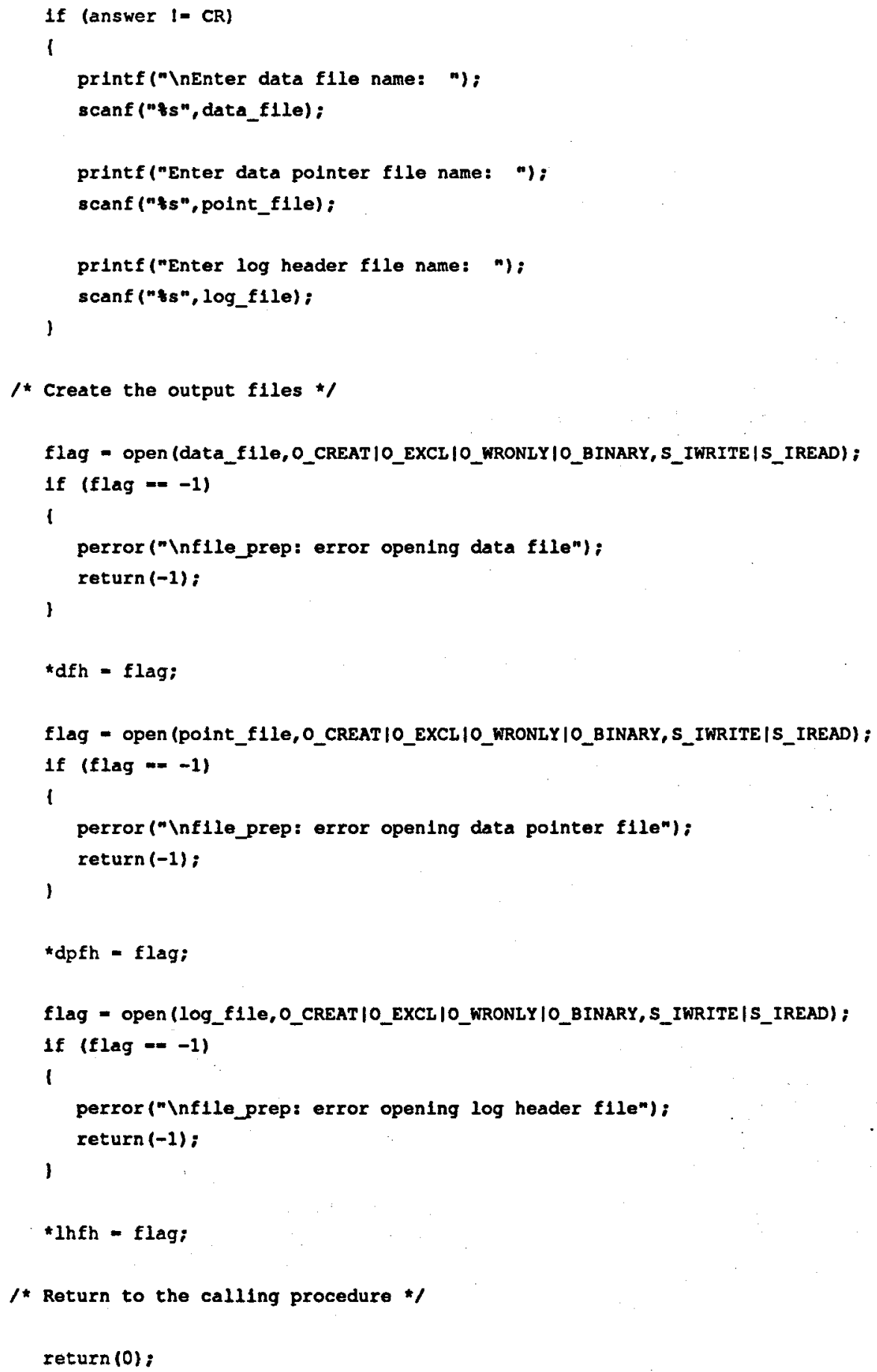




\section{Appendix F - DATASEP Source Code}

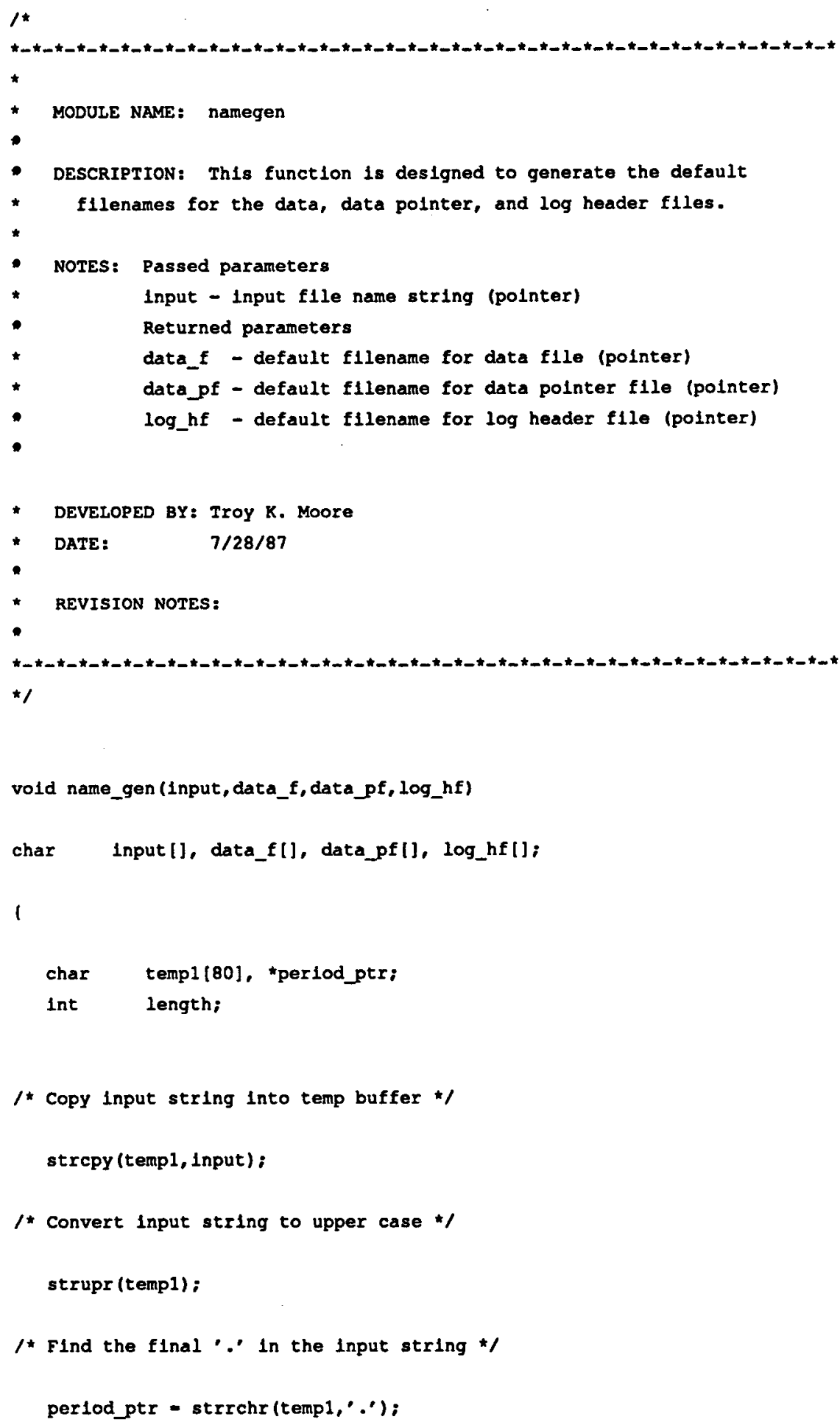




\section{Appendix F - DATASEP Source Code}

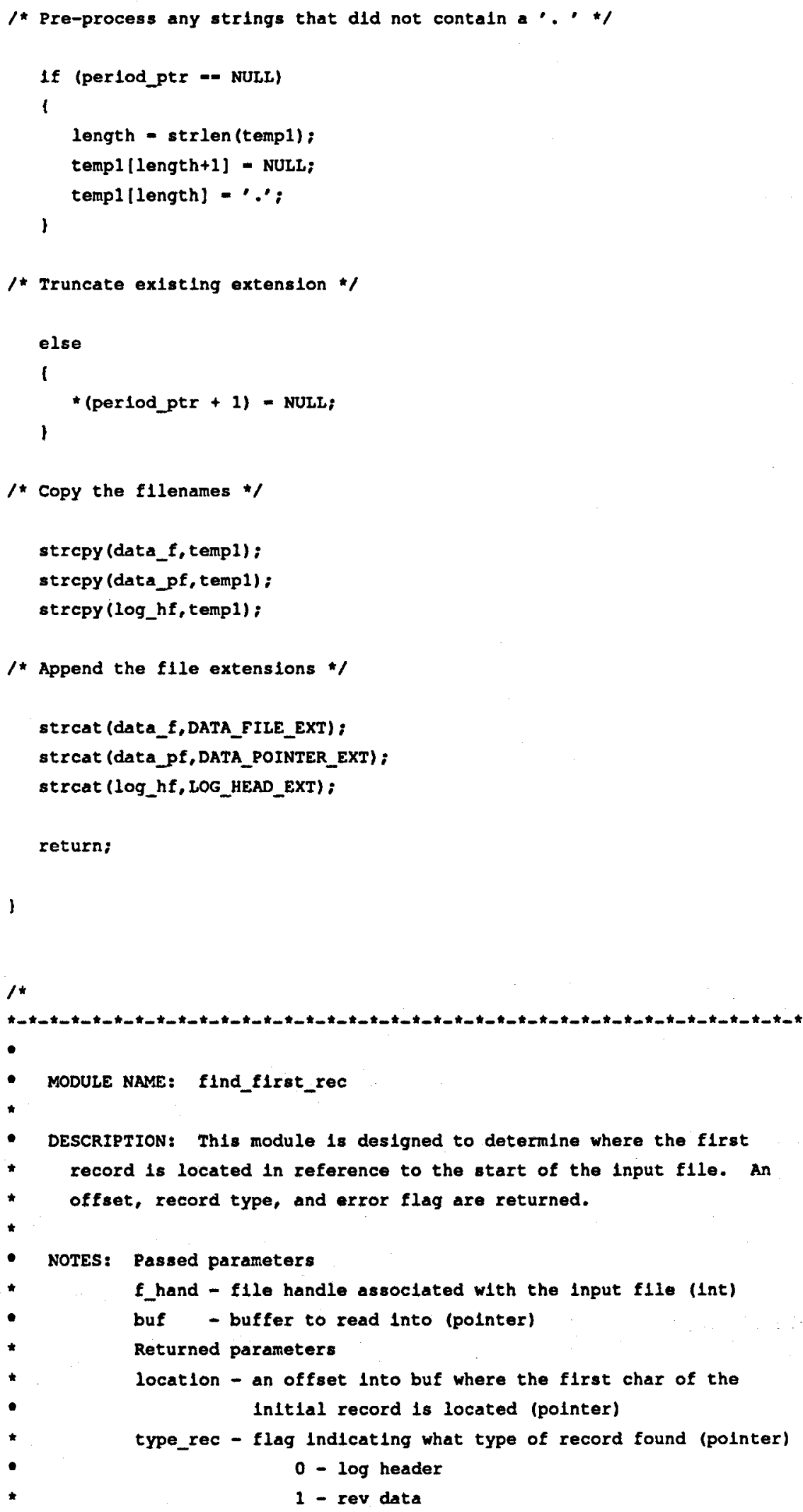




\section{Appendix F - DATASEP Source Code}

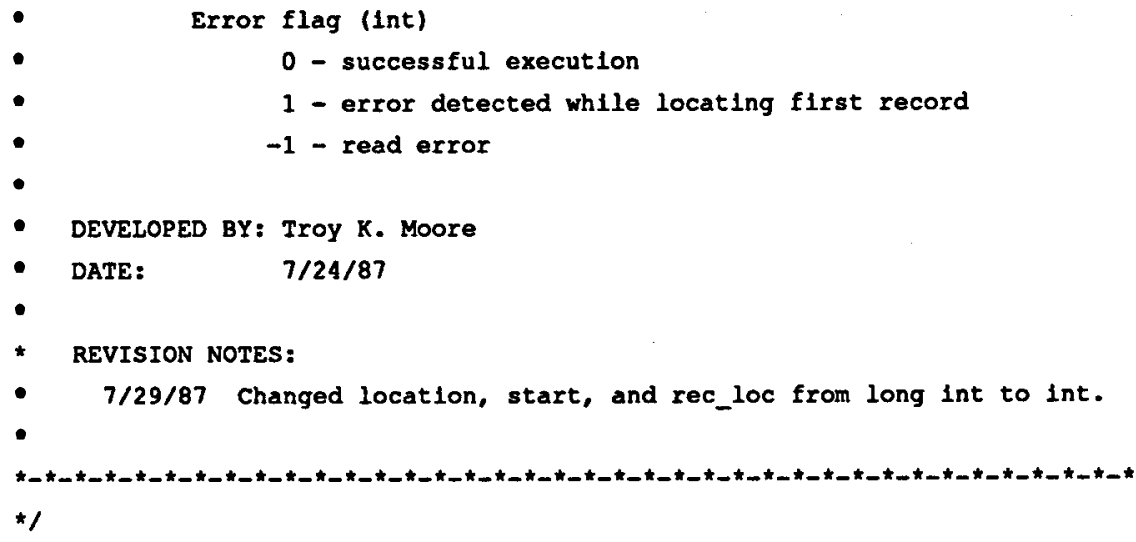




\section{Appendix F - DATASEP Source Code}

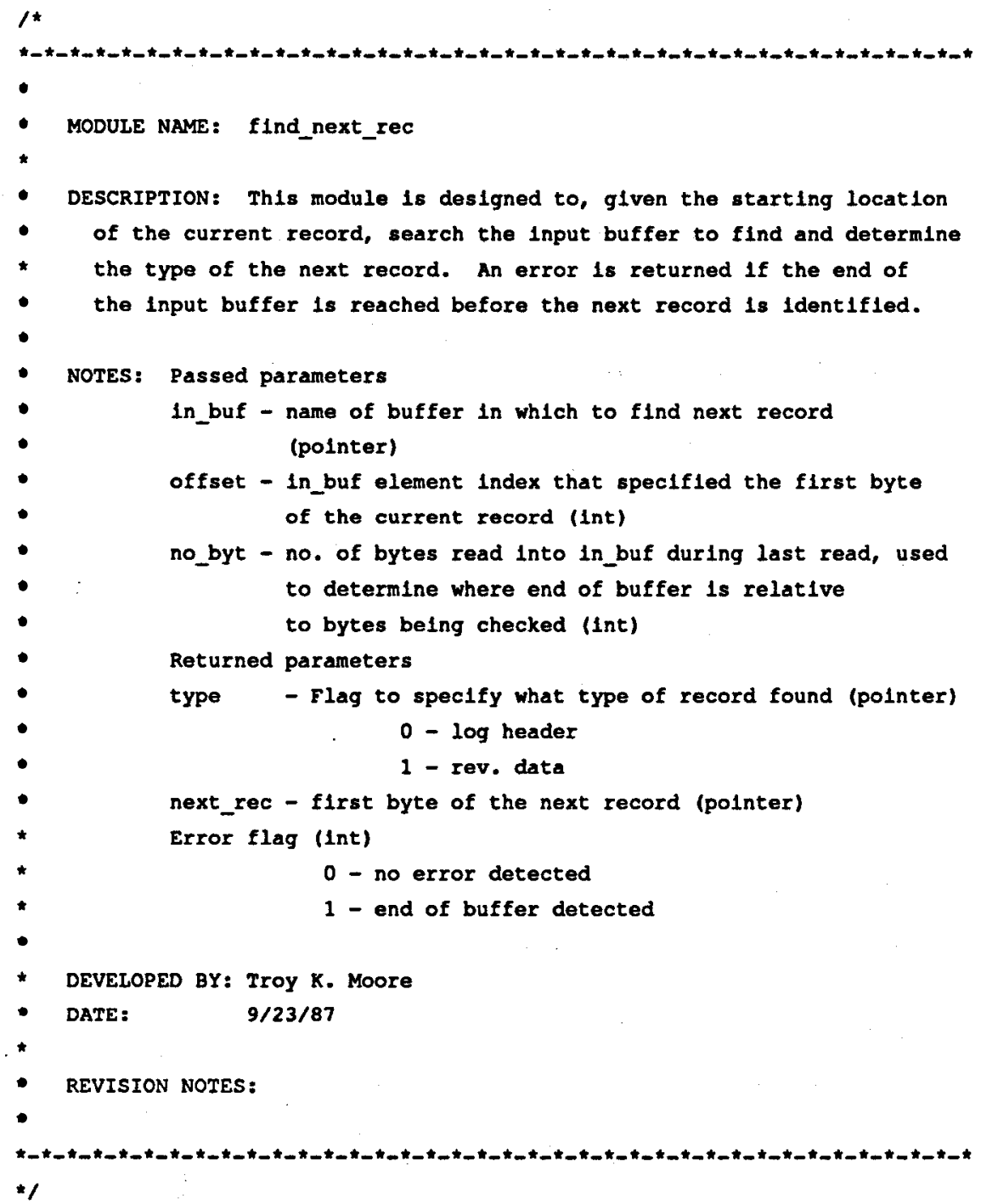

Int find_next_rec(In_buf, of fset, no_byt, type, next_rec)

char In_buf []:

Int *type, no_byt, offset, *next_rec;

1

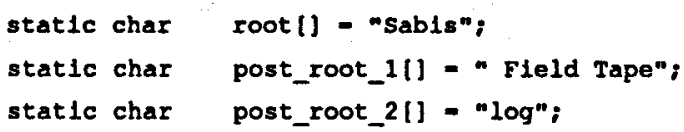




\section{Appendix F - DATASEP Source Code}

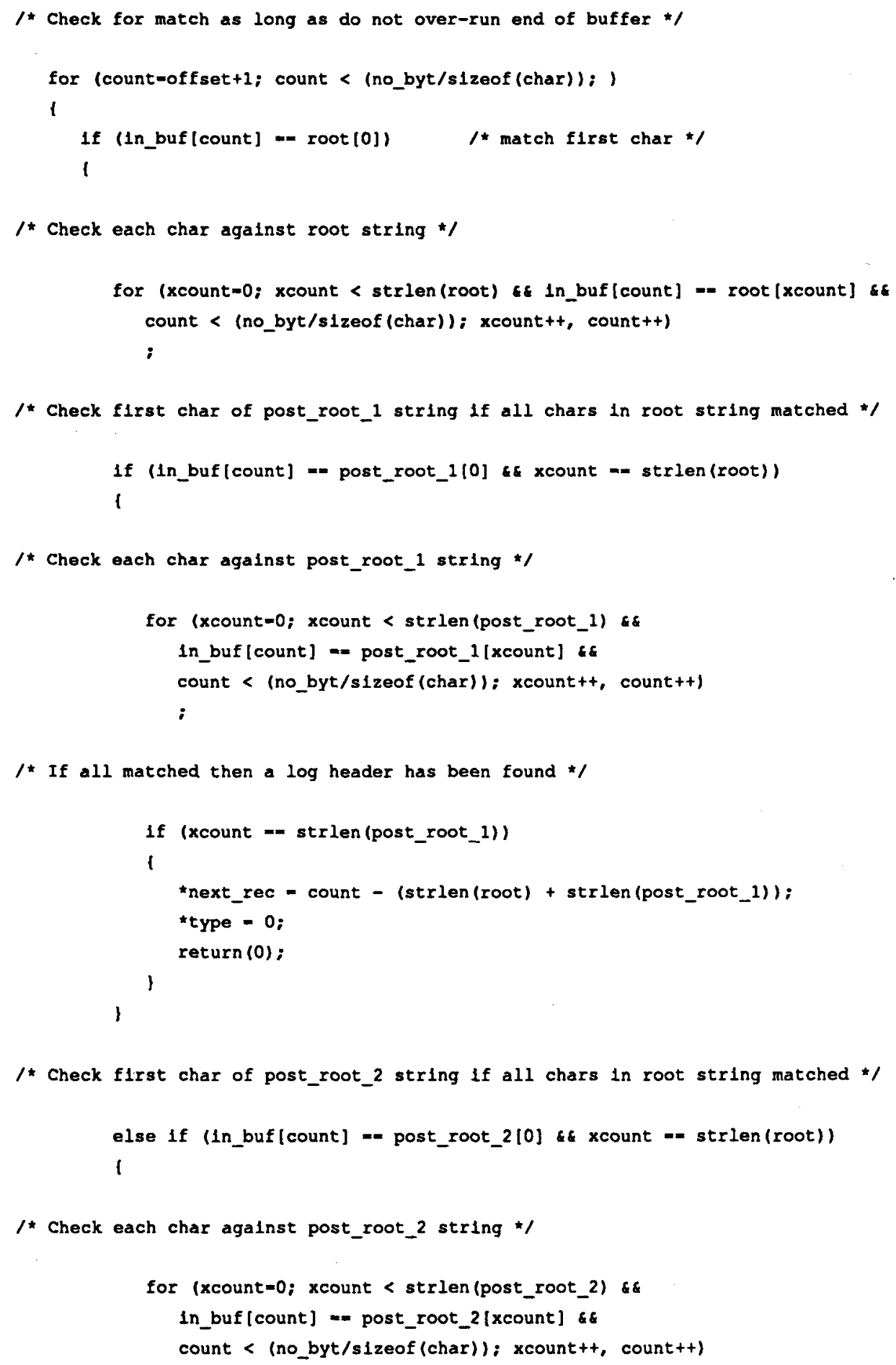




\section{Appendix F - DATASEP Source Code}

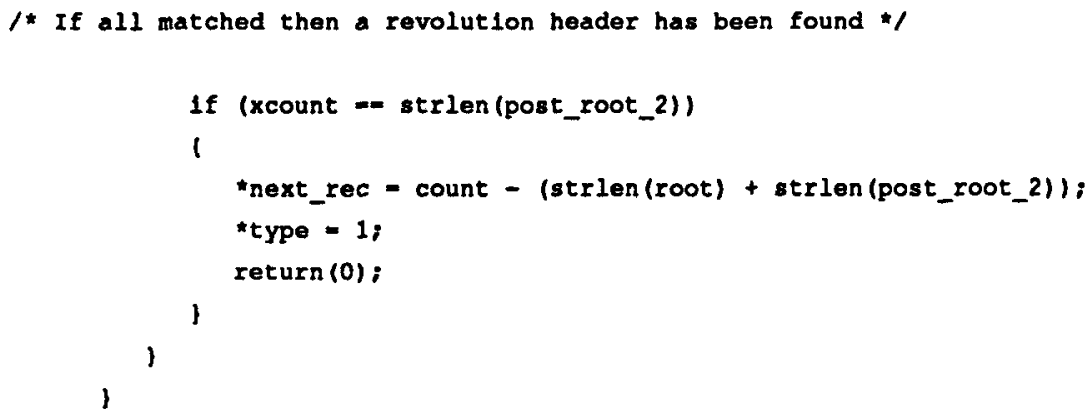




\section{Appendix F - DATASEP Source Code}

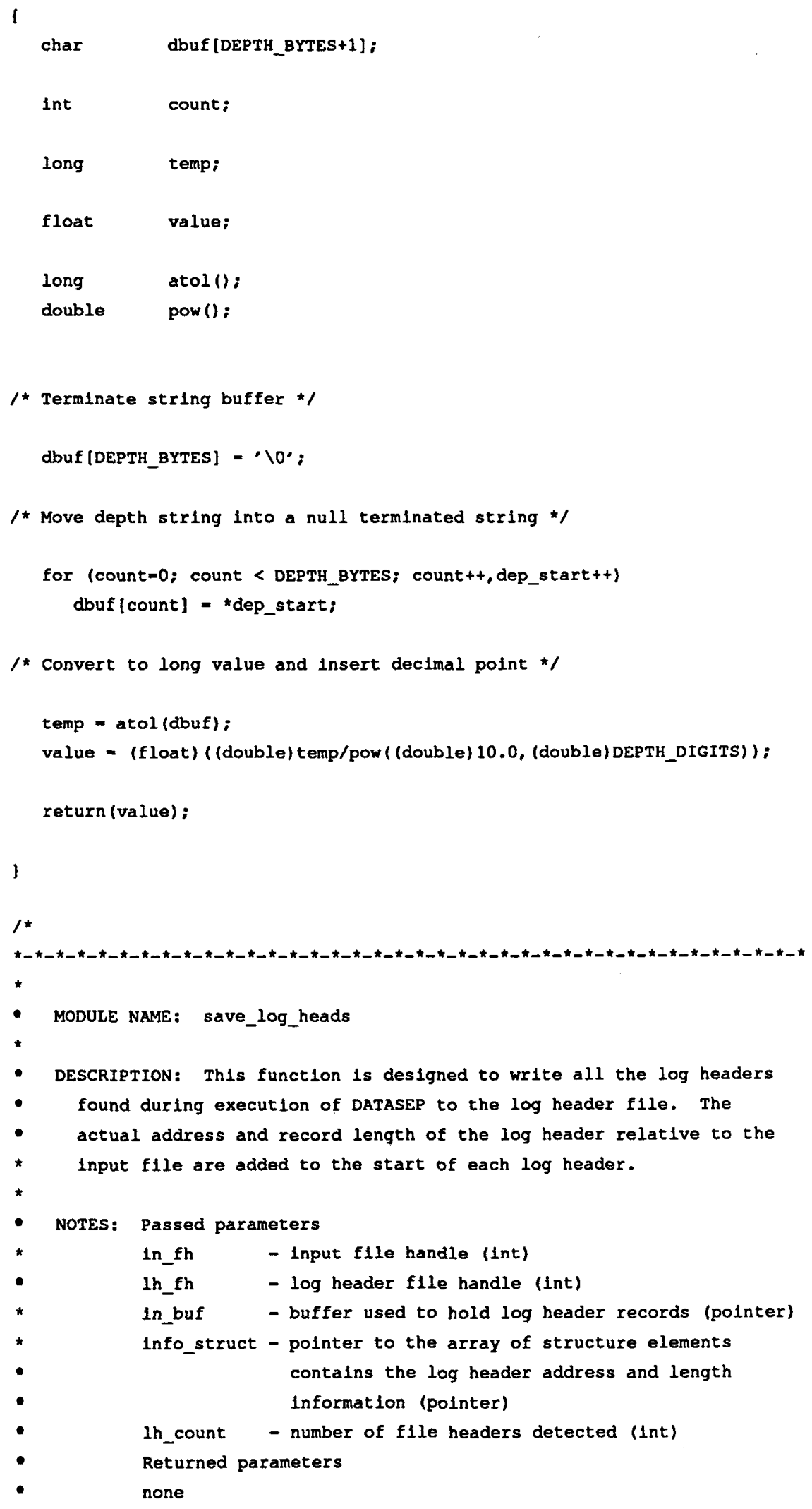




\section{Appendix F - DATASEP Source Code}

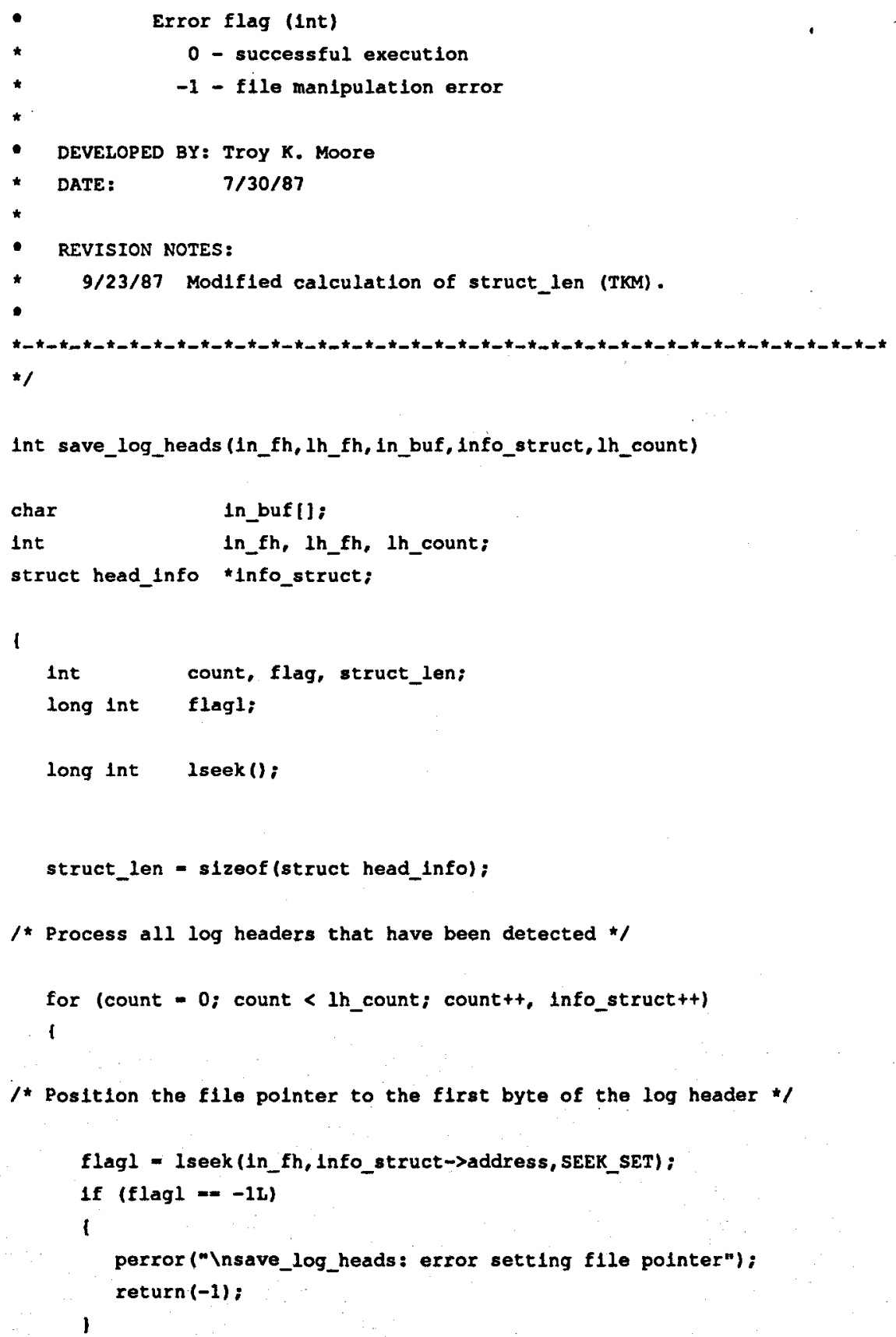

1

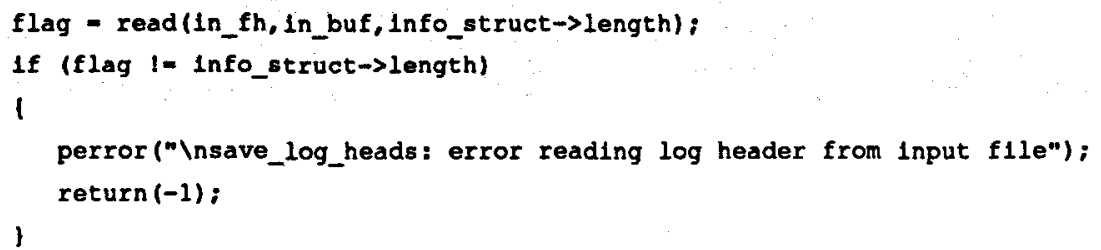




\section{Appendix F - DATASEP Source Code}

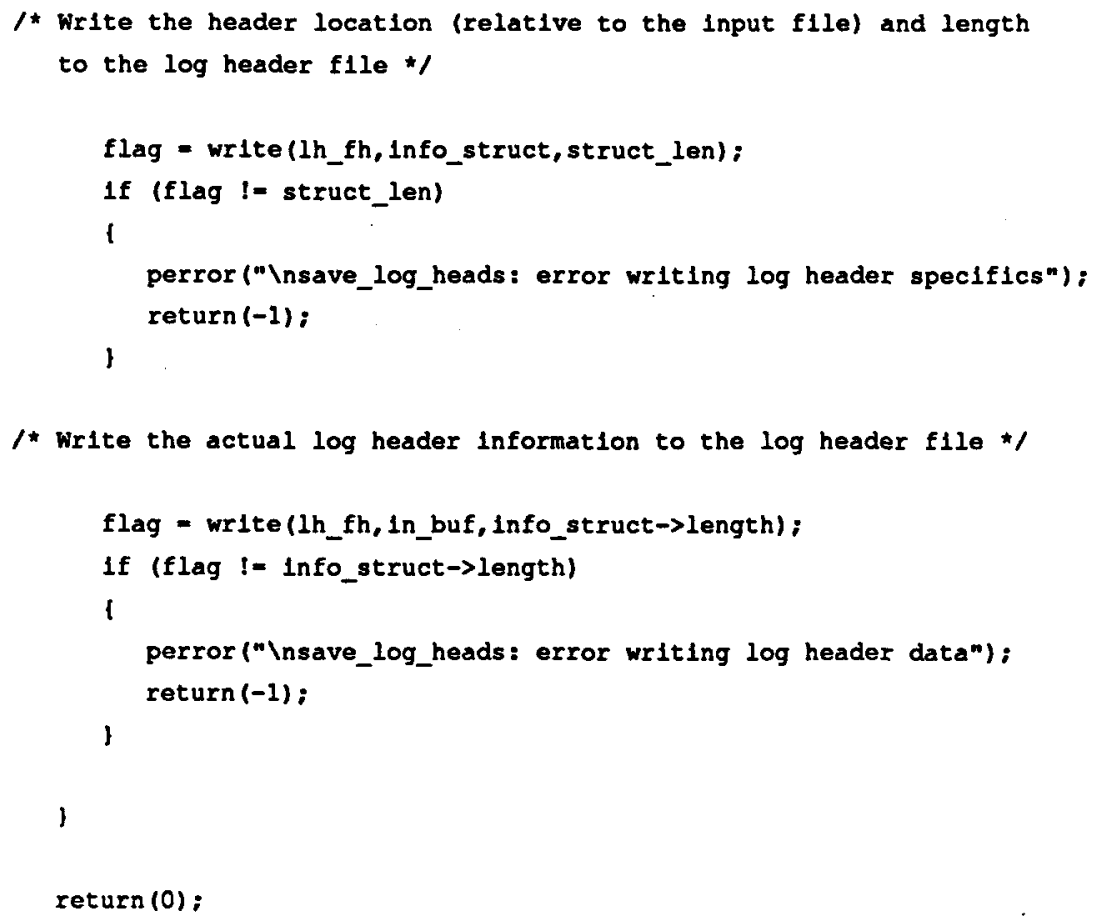




\section{Appendix G - DPDUMP Source Code}

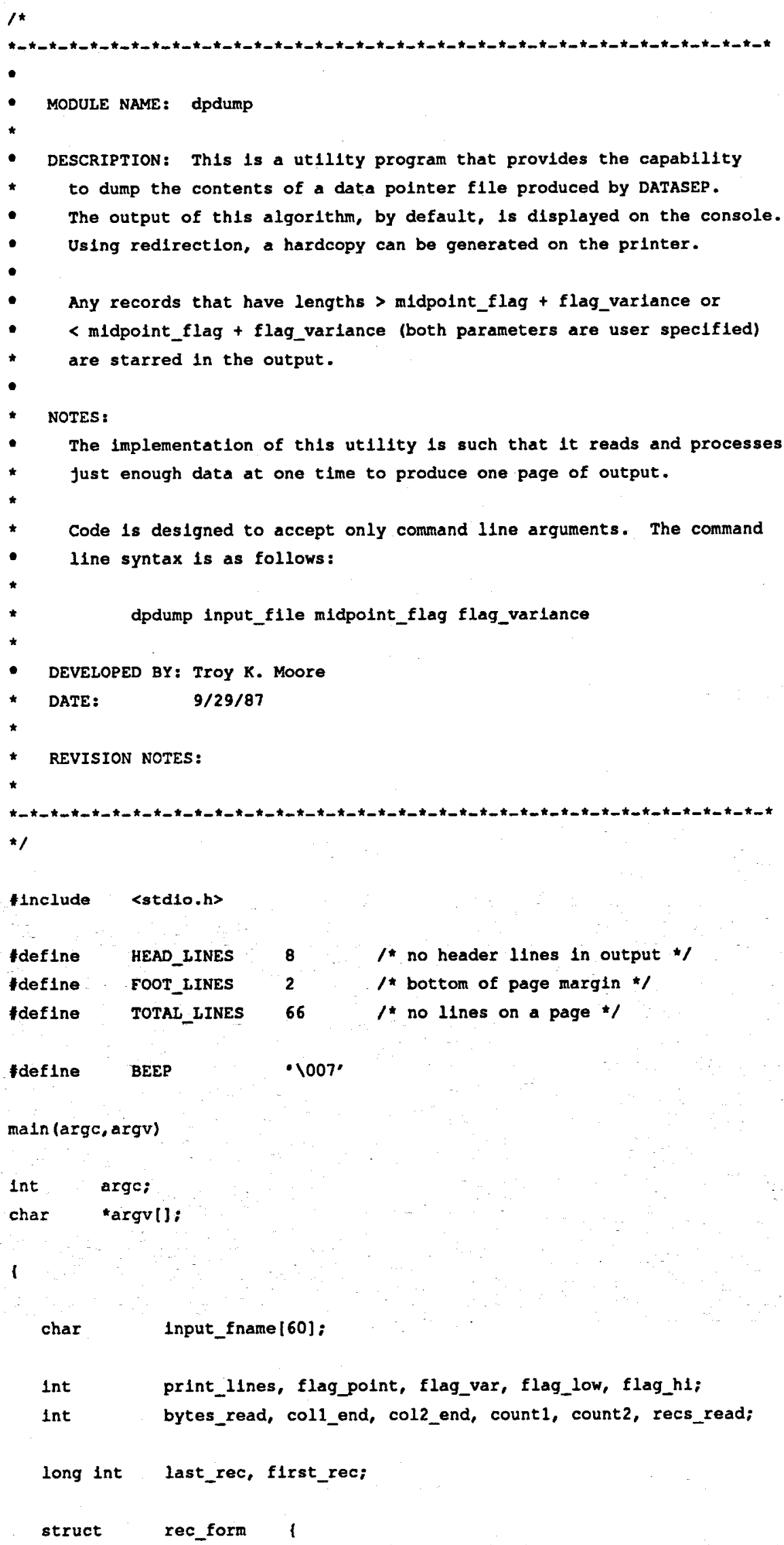




\section{Appendix G - DPDUMP Source Code}

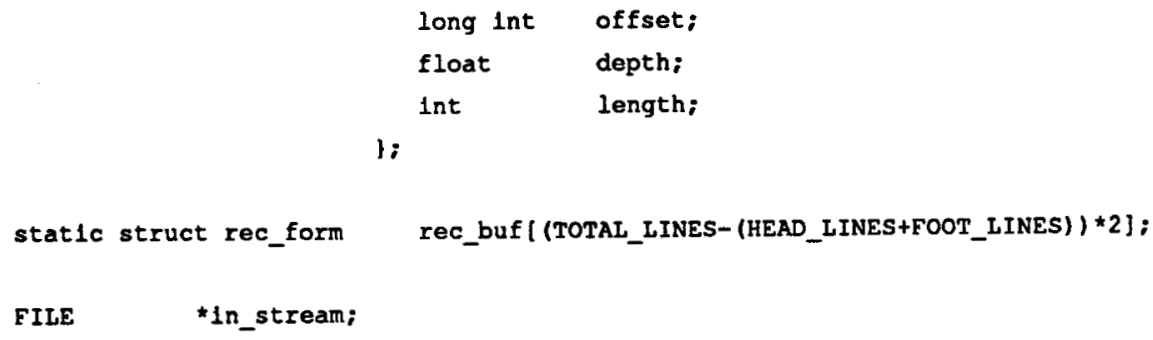




\section{Appendix G - DPDUMP Source Code}

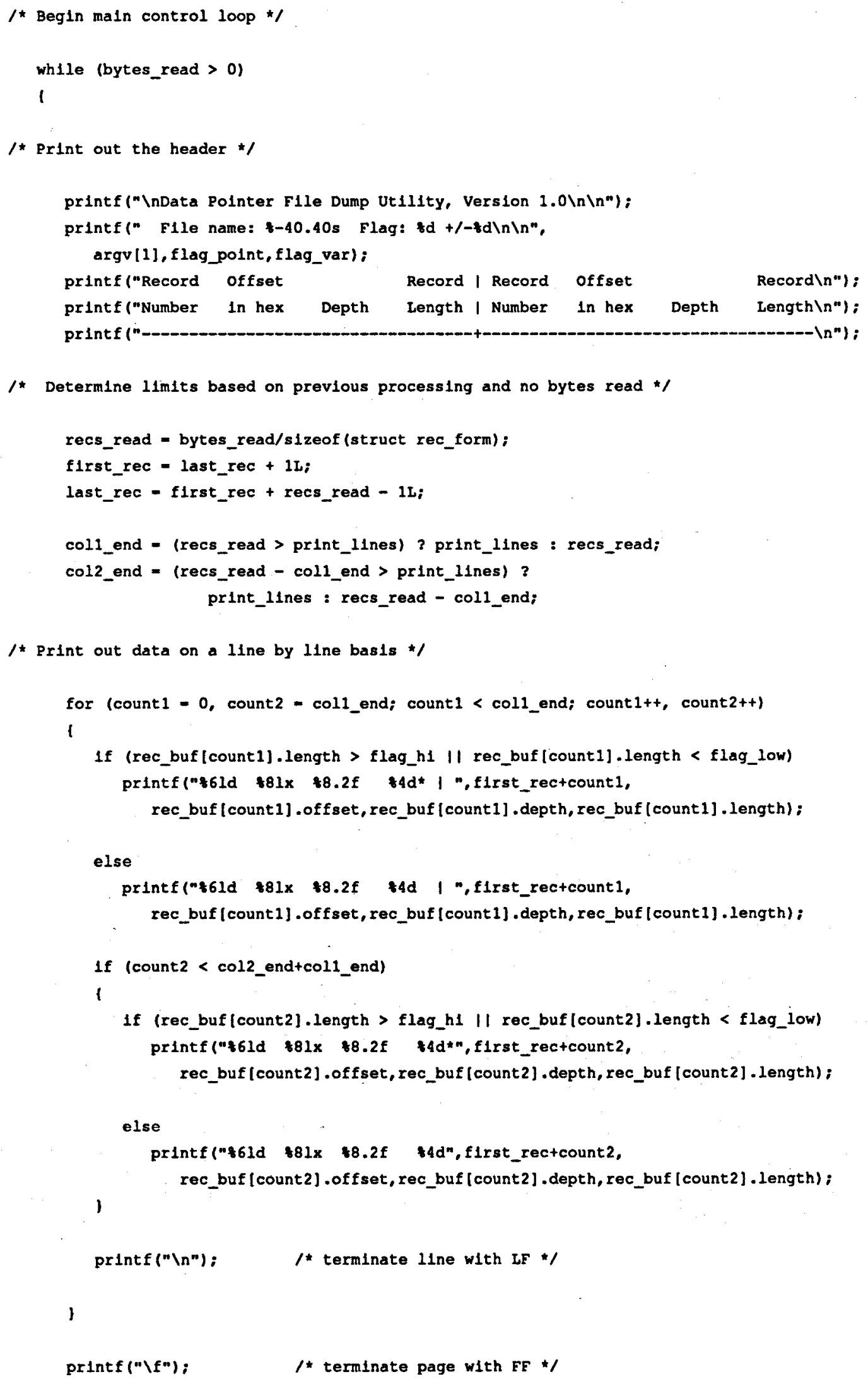




\section{Appendix G - DPDUMP Source Code}

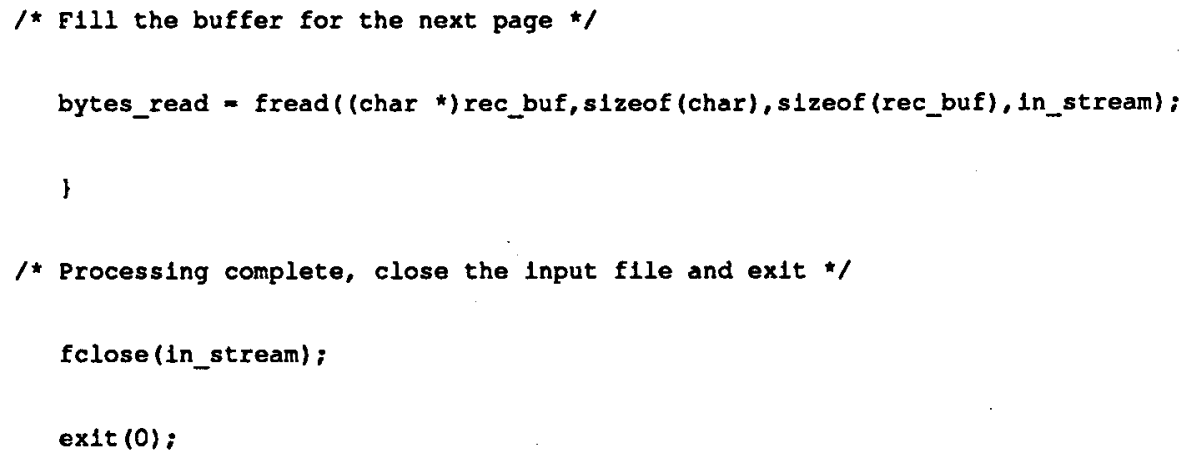




\section{Appendix H - BDINVRT Source Code}

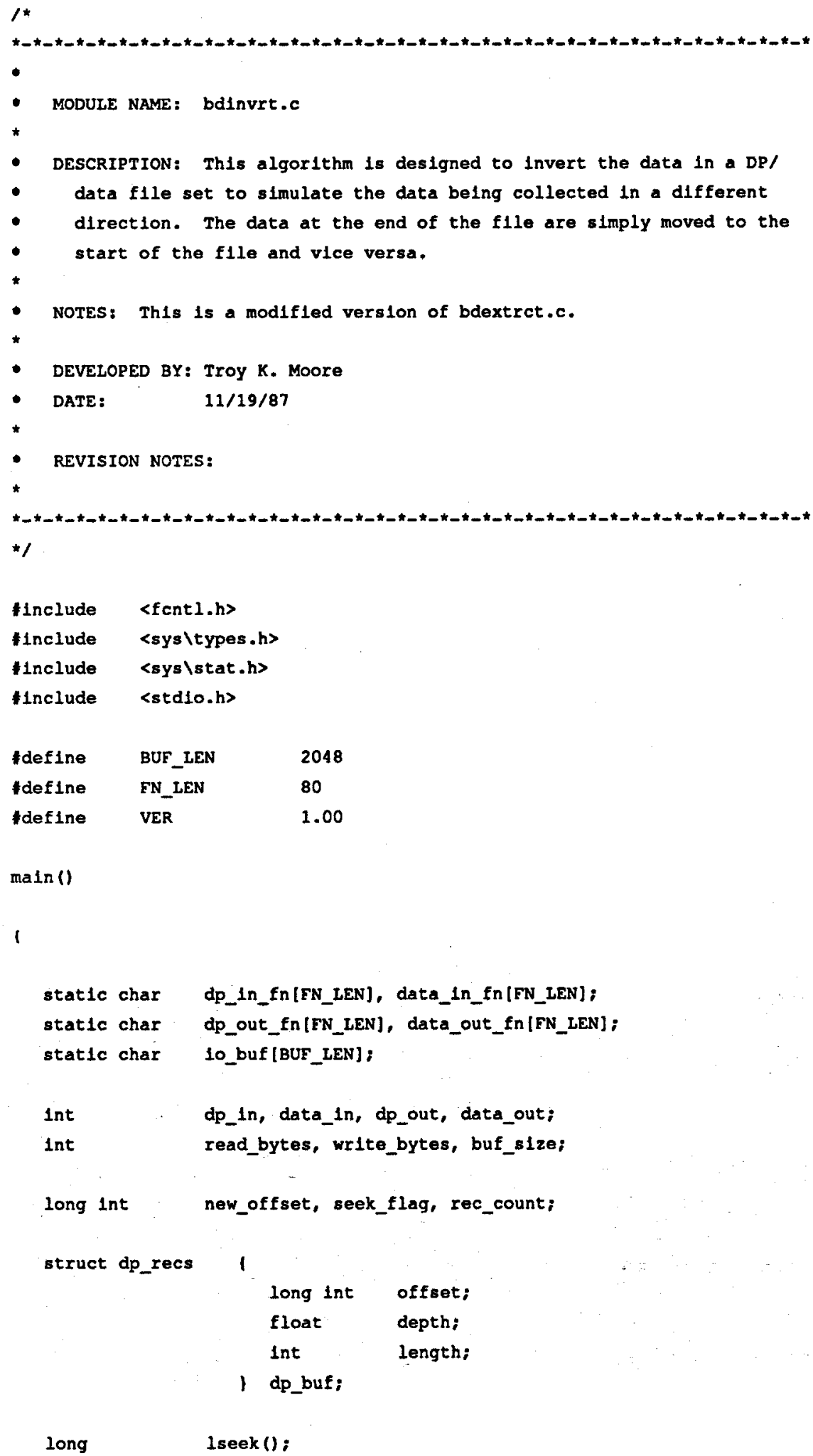

\begin{tabular}{|c|c|c|}
\hline Include & $\langle$ fentl.h $\rangle$ & \\
\hline Hinclude & 〈sysltypes .h〉 & \\
\hline Include & 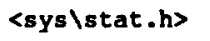 & \\
\hline H Include & <stdio.h> & \\
\hline tdefine & BUF_IEN & 2048 \\
\hline tefine & FN_LEN & 80 \\
\hline ldefine & VER & 1.00 \\
\hline
\end{tabular}

$\operatorname{mann}()$

I 


\section{Appendix H - BDINVRT Source Code}

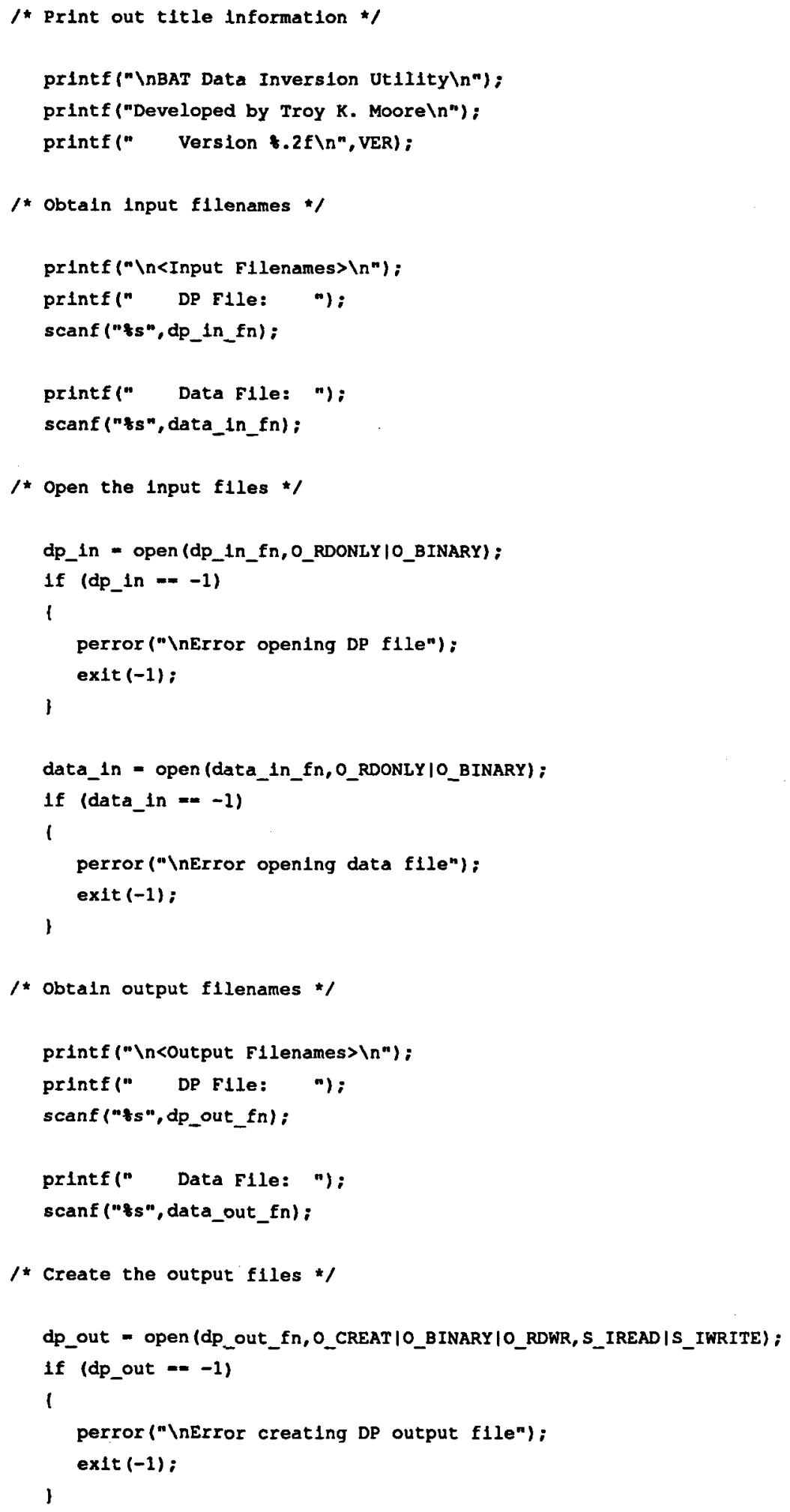




\section{Appendix H - BDINVRT Source Code}

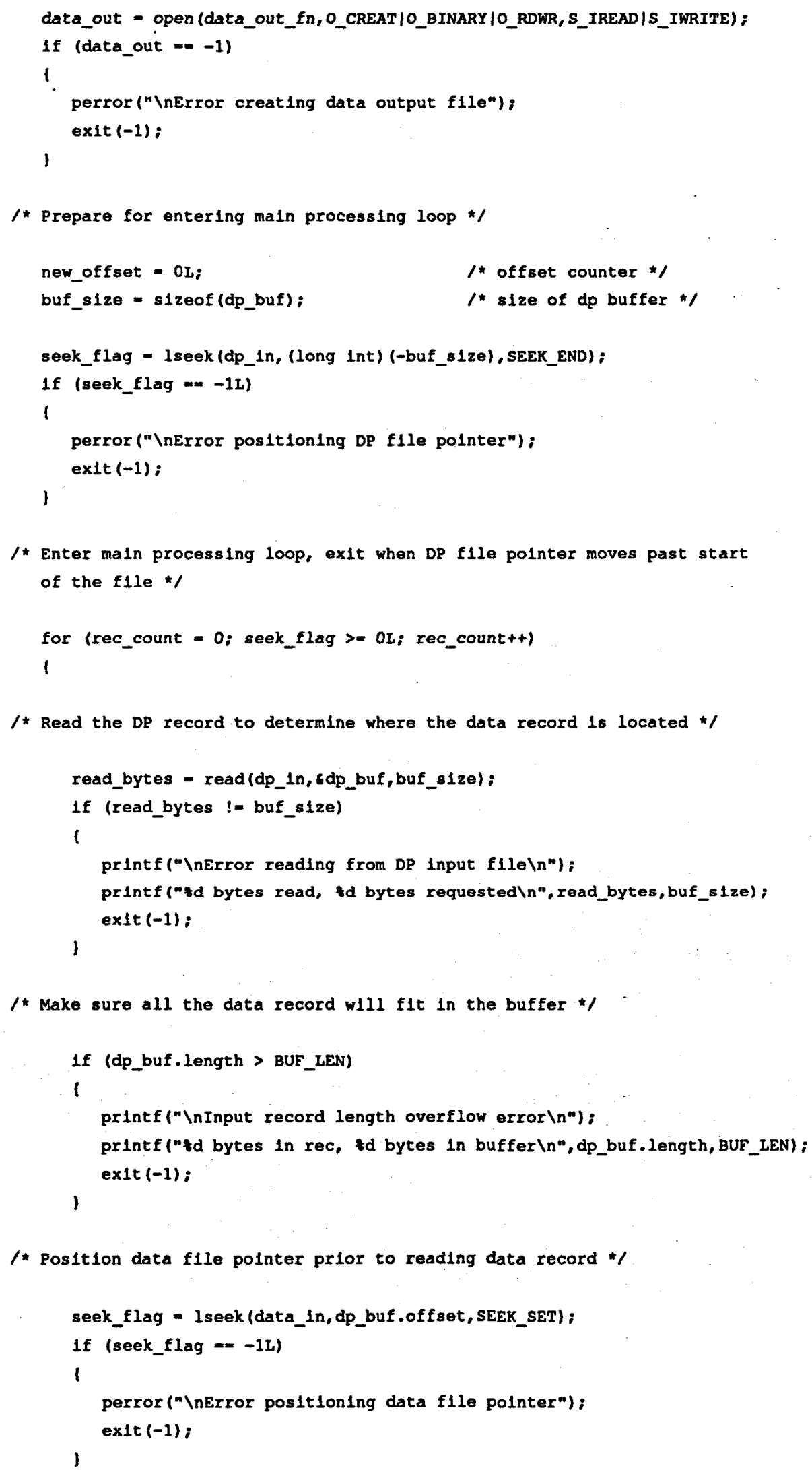




\section{Appendix H - BDINVRT Source Code}

$1 *$ Read the data record $\star /$

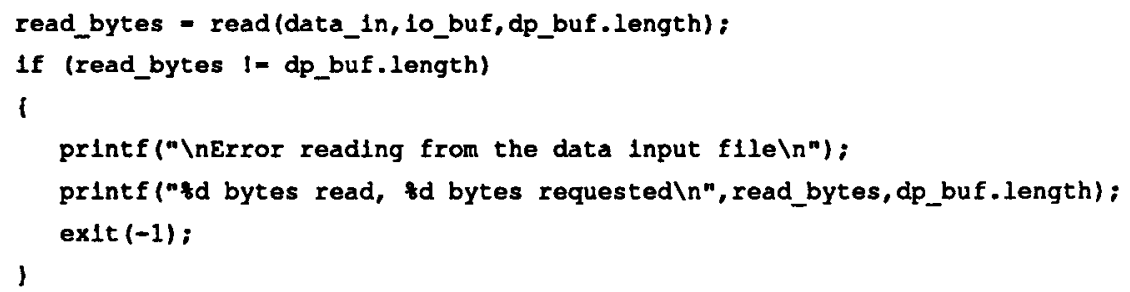




\section{Appendix I - BDEXTRCT Source Code}

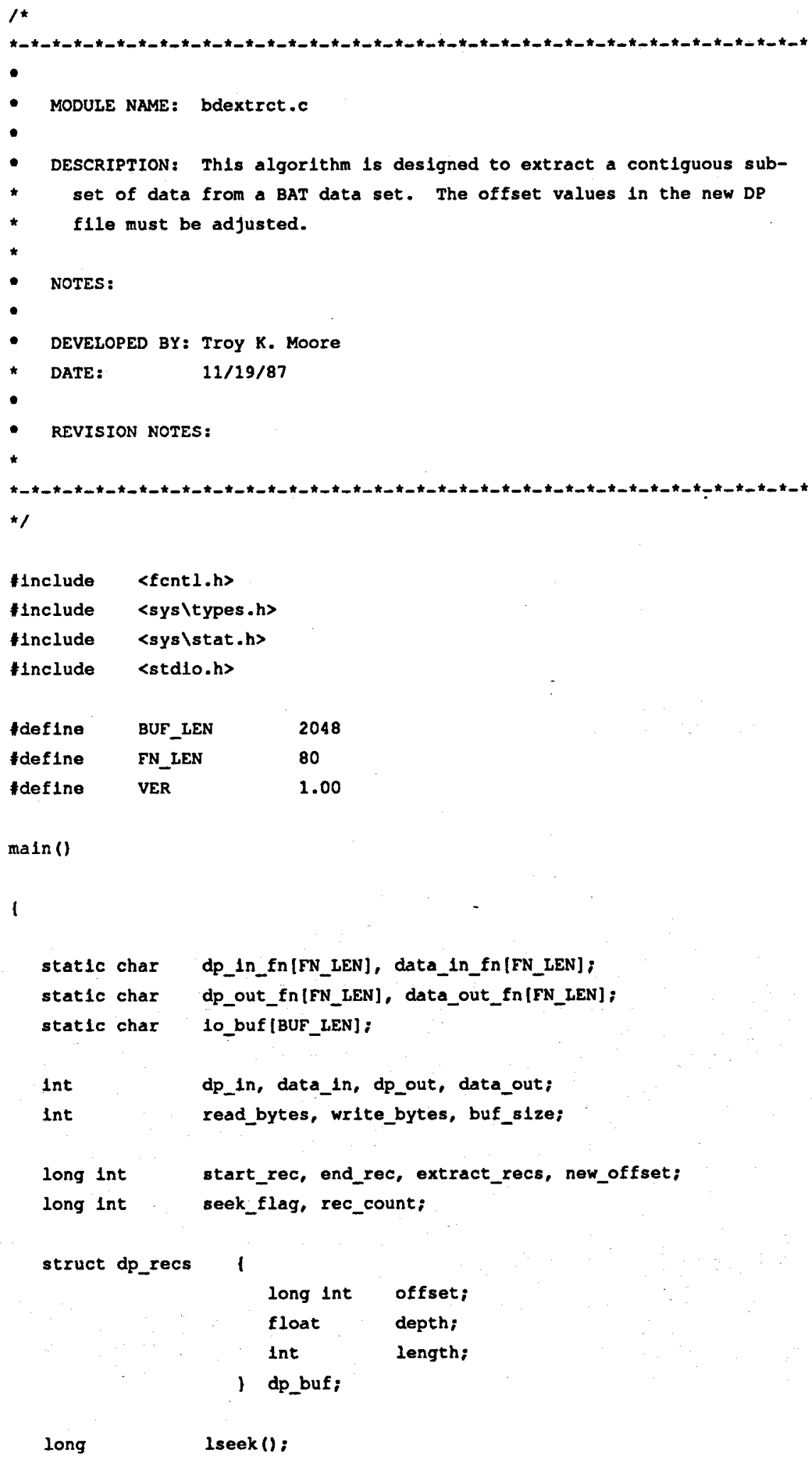




\section{Appendix I - BDEXTRCT Source Code}

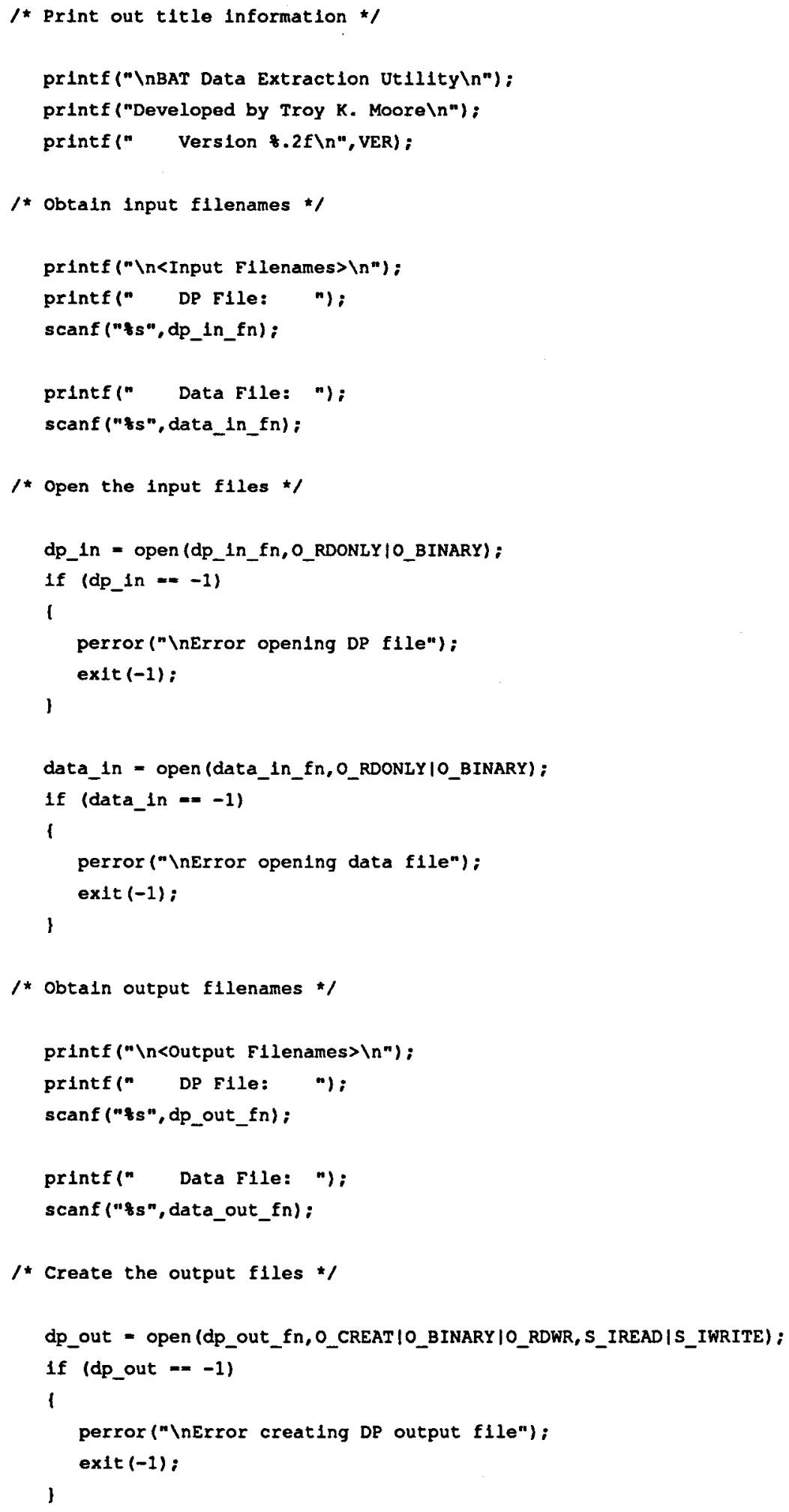




\section{Appendix I - BDEXTRCT Source Code}

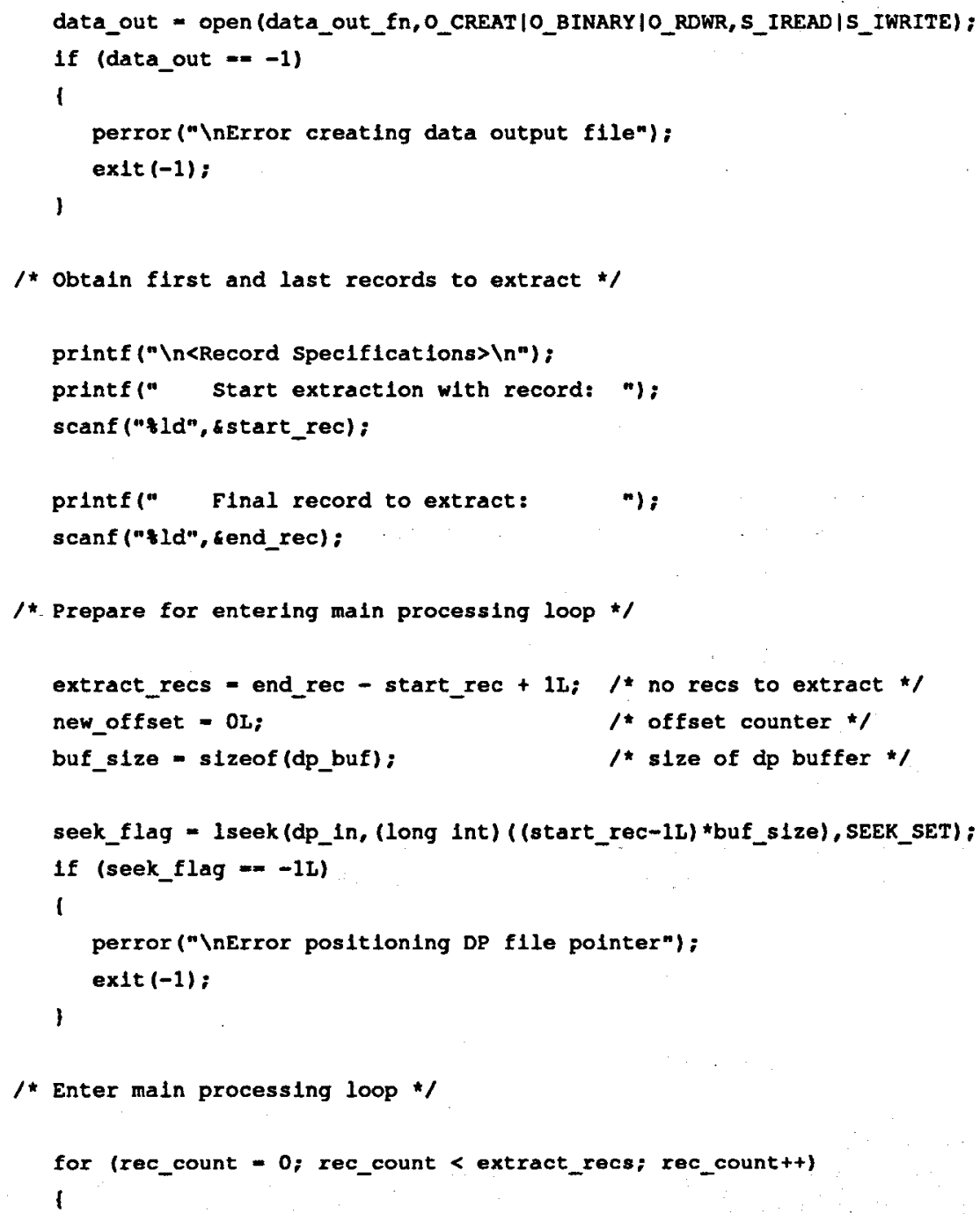




\section{Appendix I - BDEXTRCT Source Code}

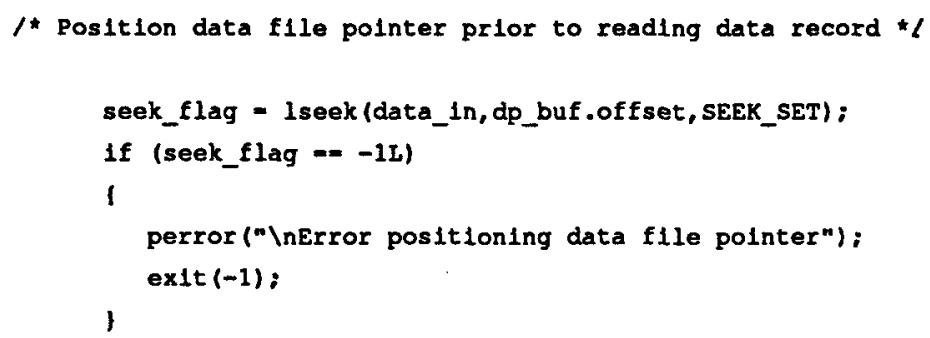




\section{Appendix I - BDEXTRCT Source Code}

exit (0):

1 


\section{Appendix J - BATD Source Code}

F1le: BATDDEFS.H

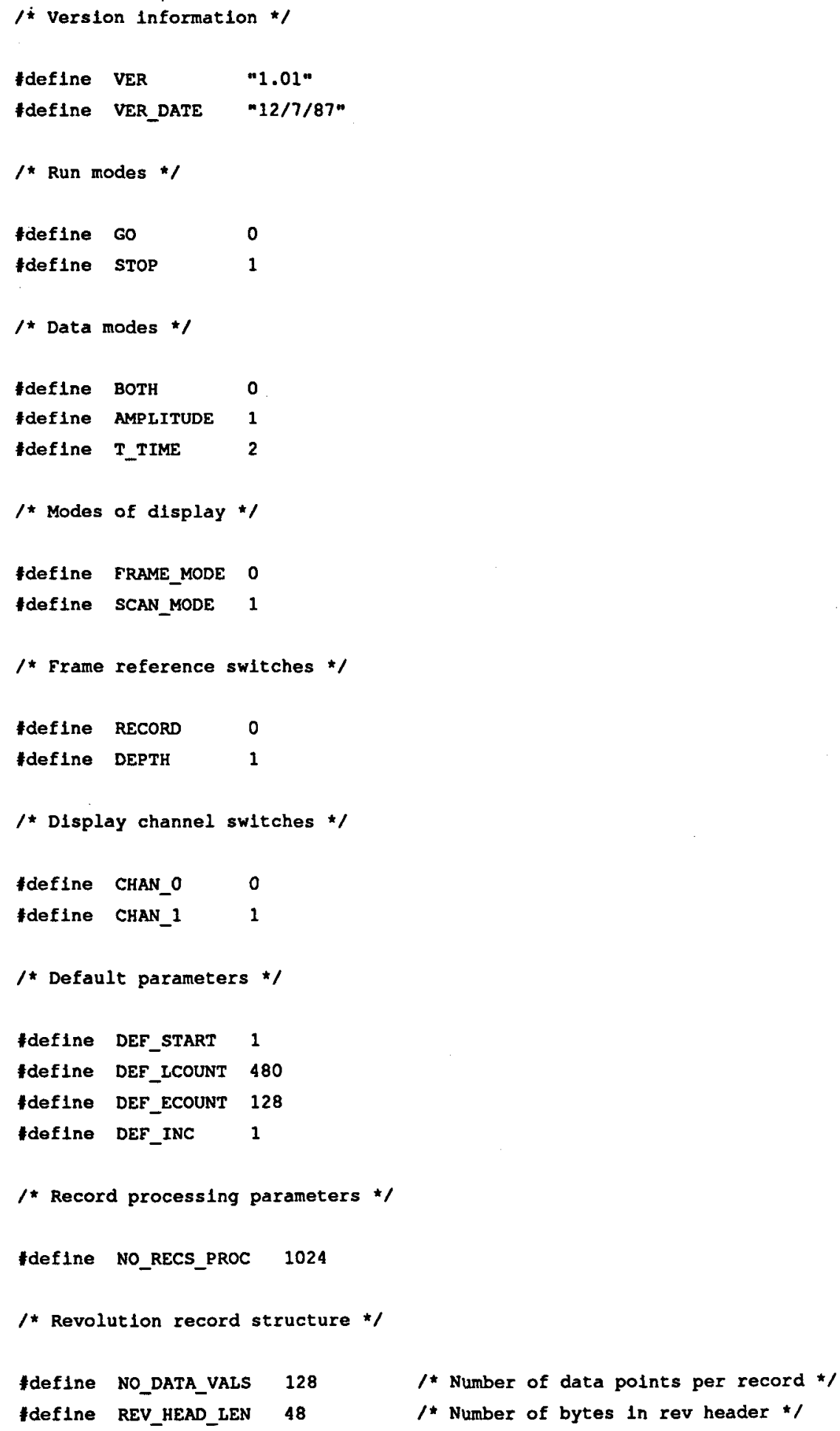




\section{Appendix J - BATD Source Code}

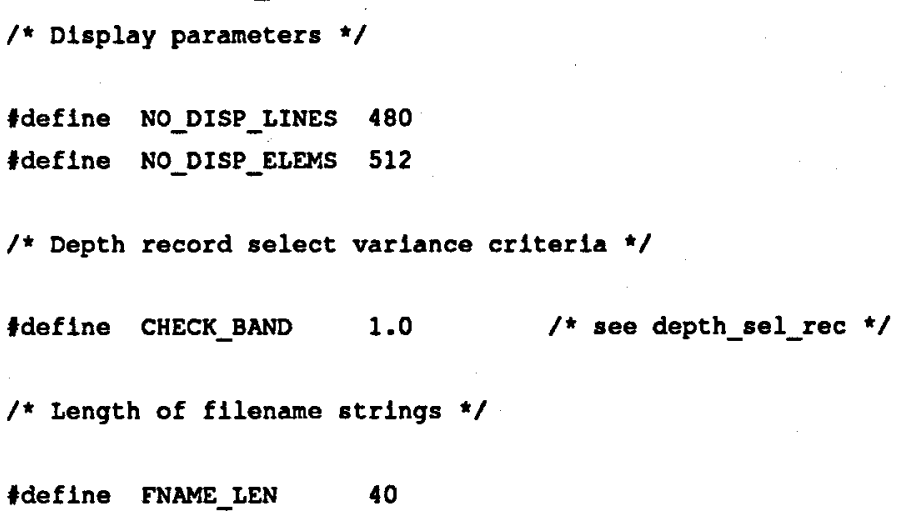




\section{Appendix J - BATD Source Code}

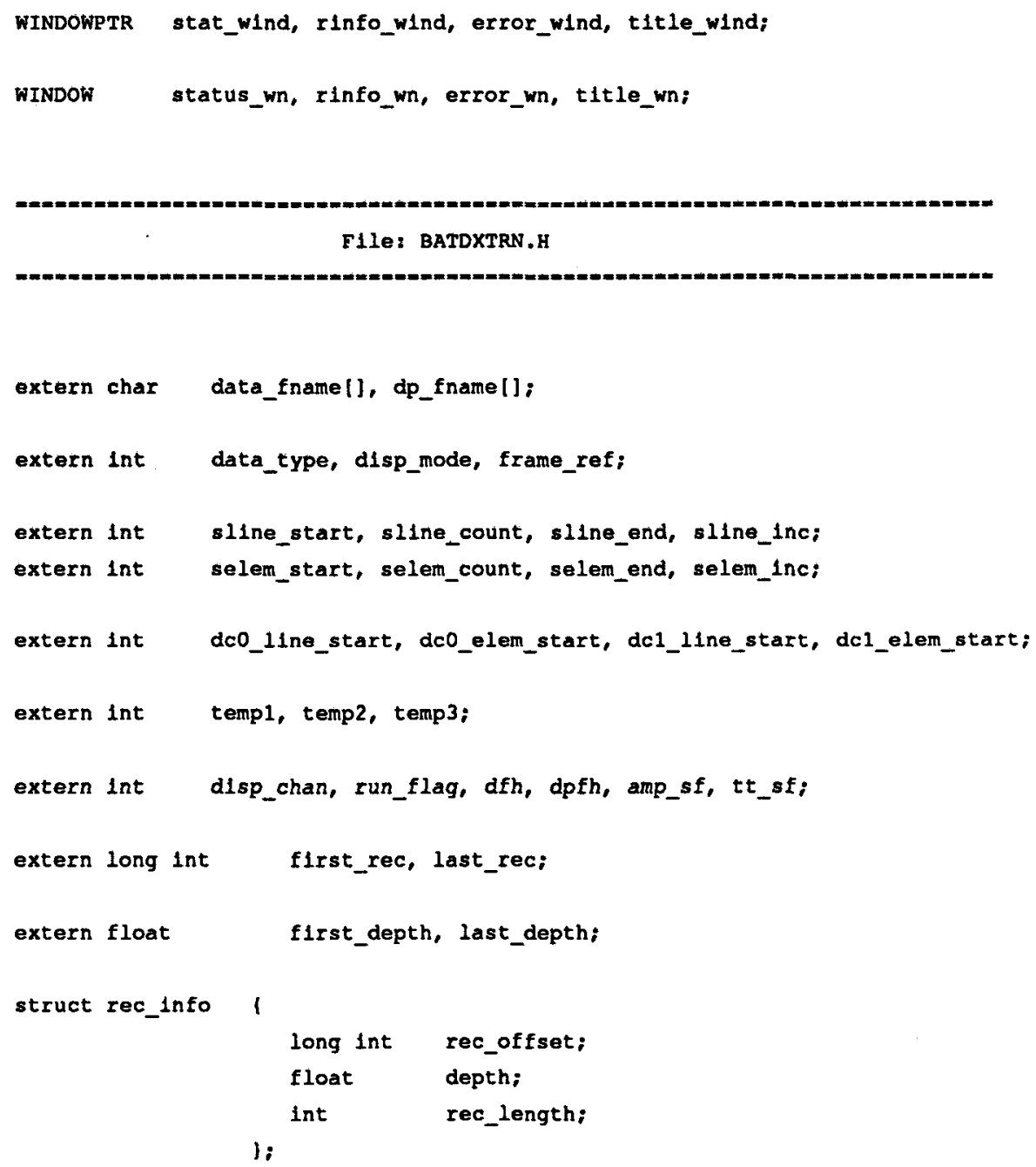




\section{Appendix J - BATD Source Code}

File: BATD.C

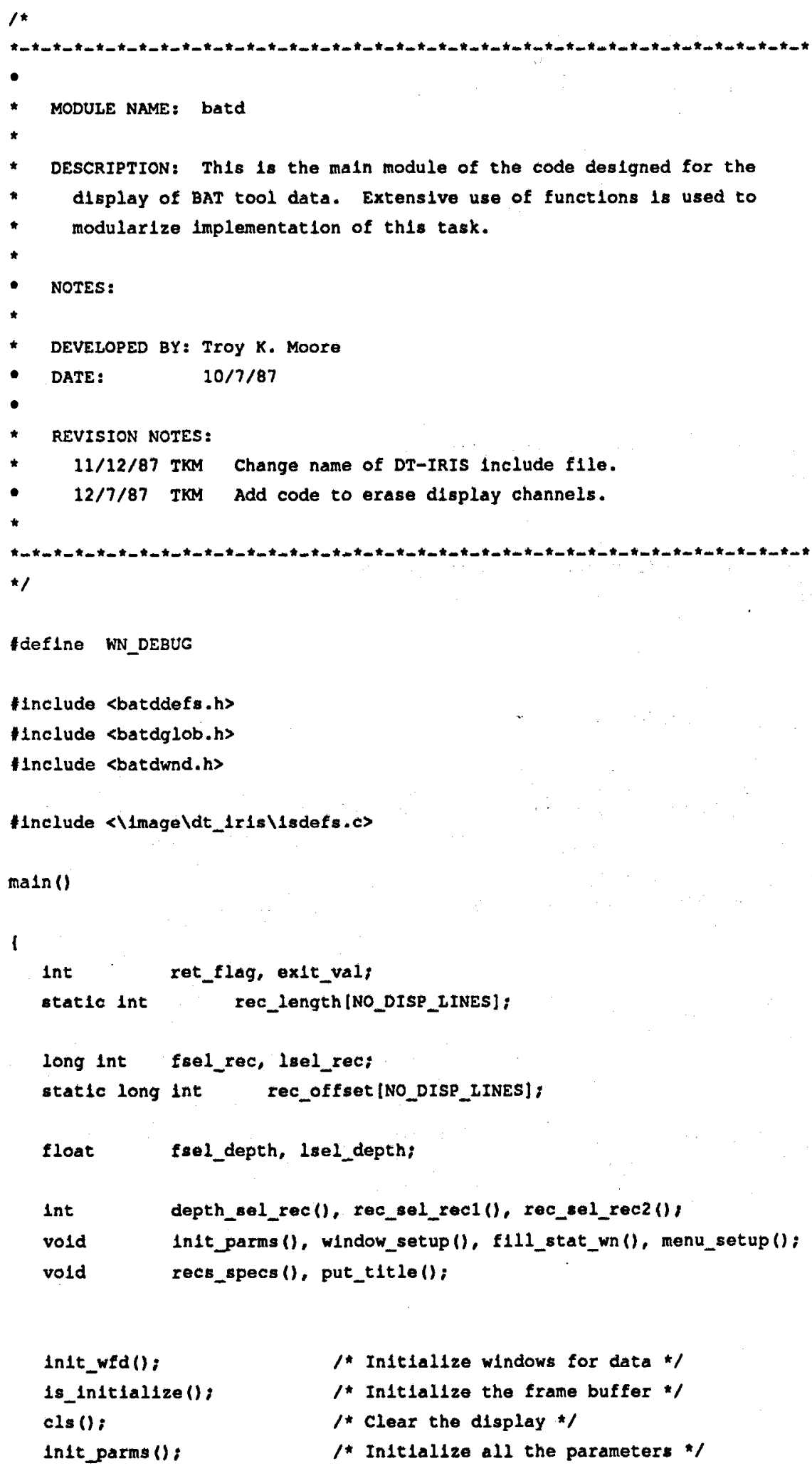




\section{Appendix J - BATD Source Code}

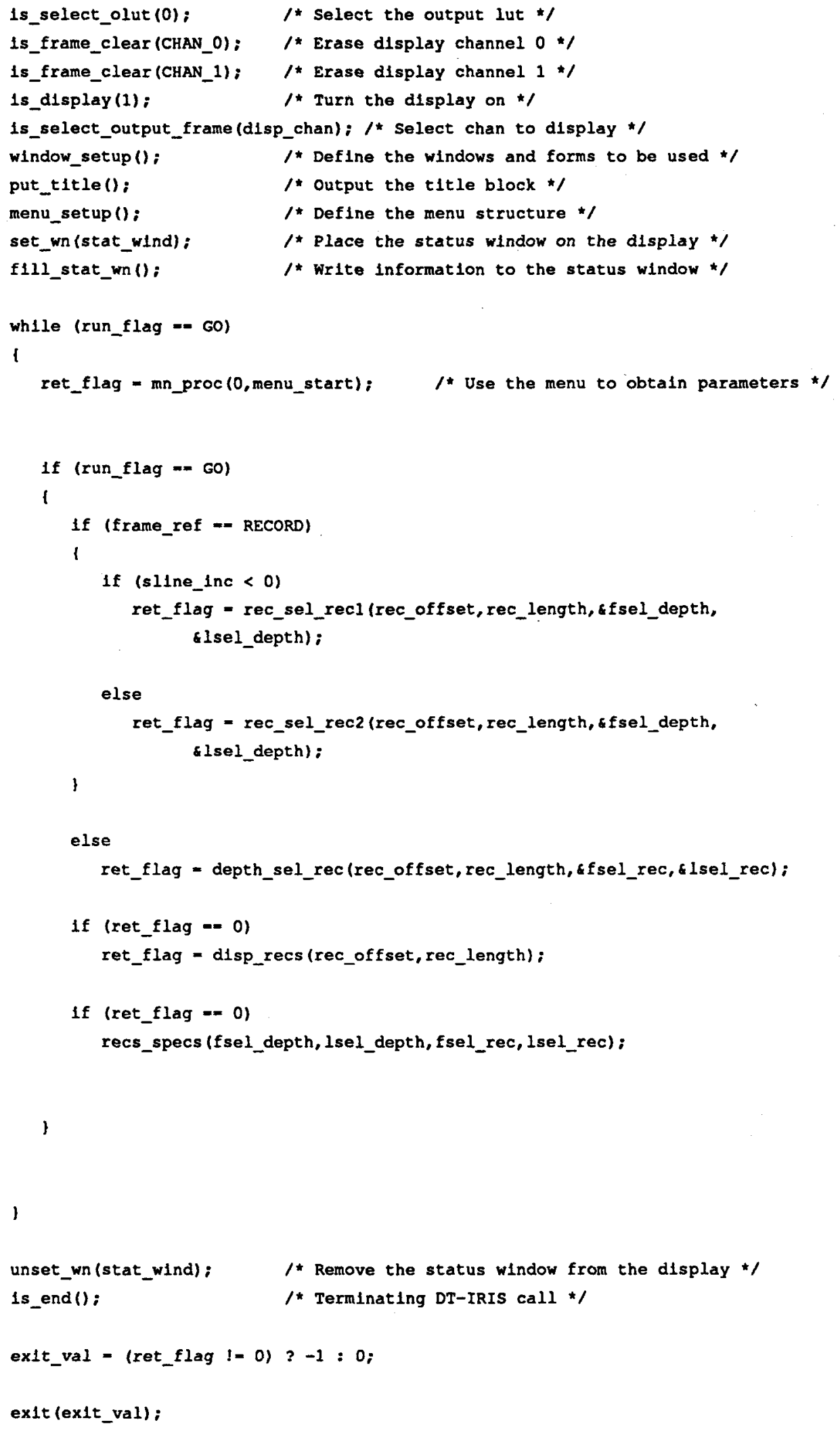




\section{Appendix J - BATD Source Code}

File: BATDSTUP.C

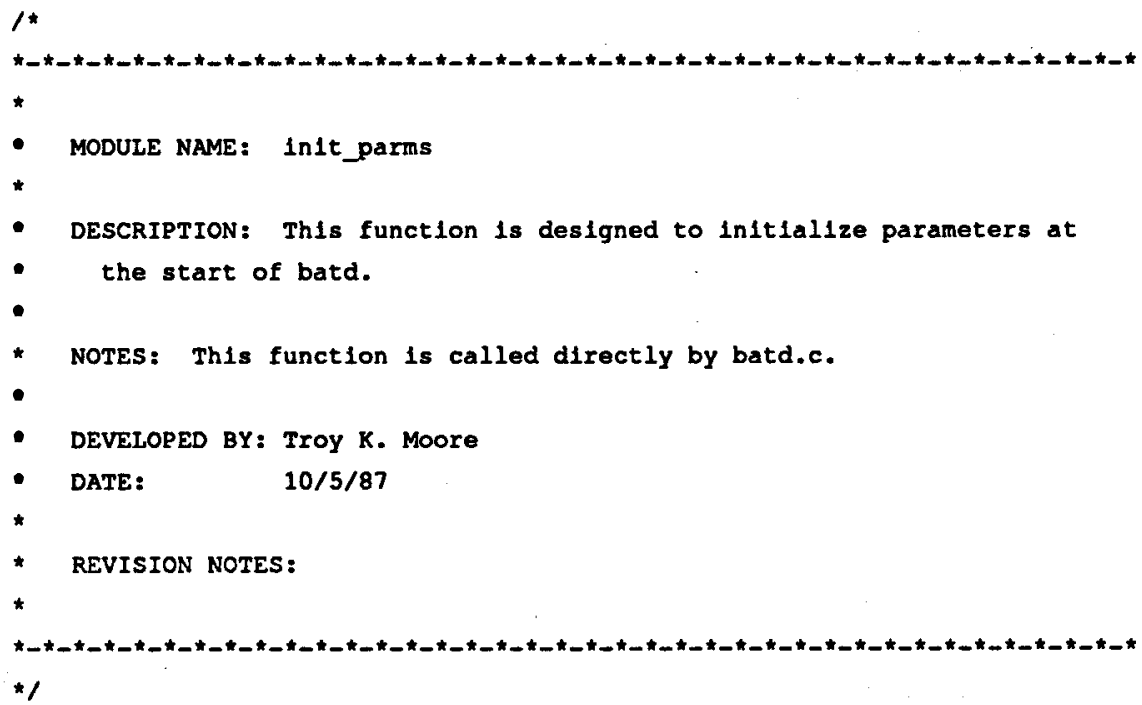




\section{Appendix J • BATD Source Code}

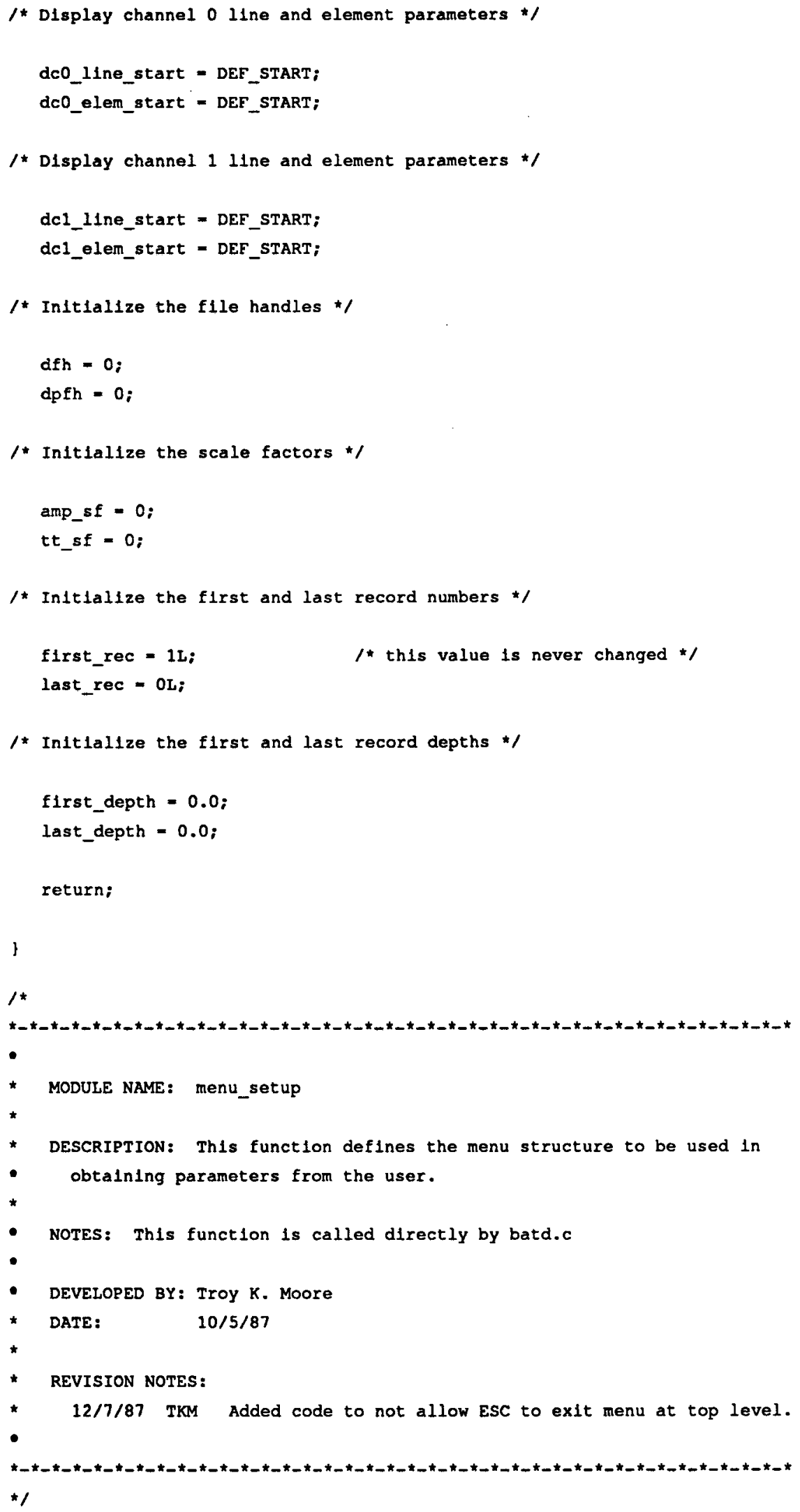




\section{Appendix J - BATD Source Code}

vold menu_setup ()

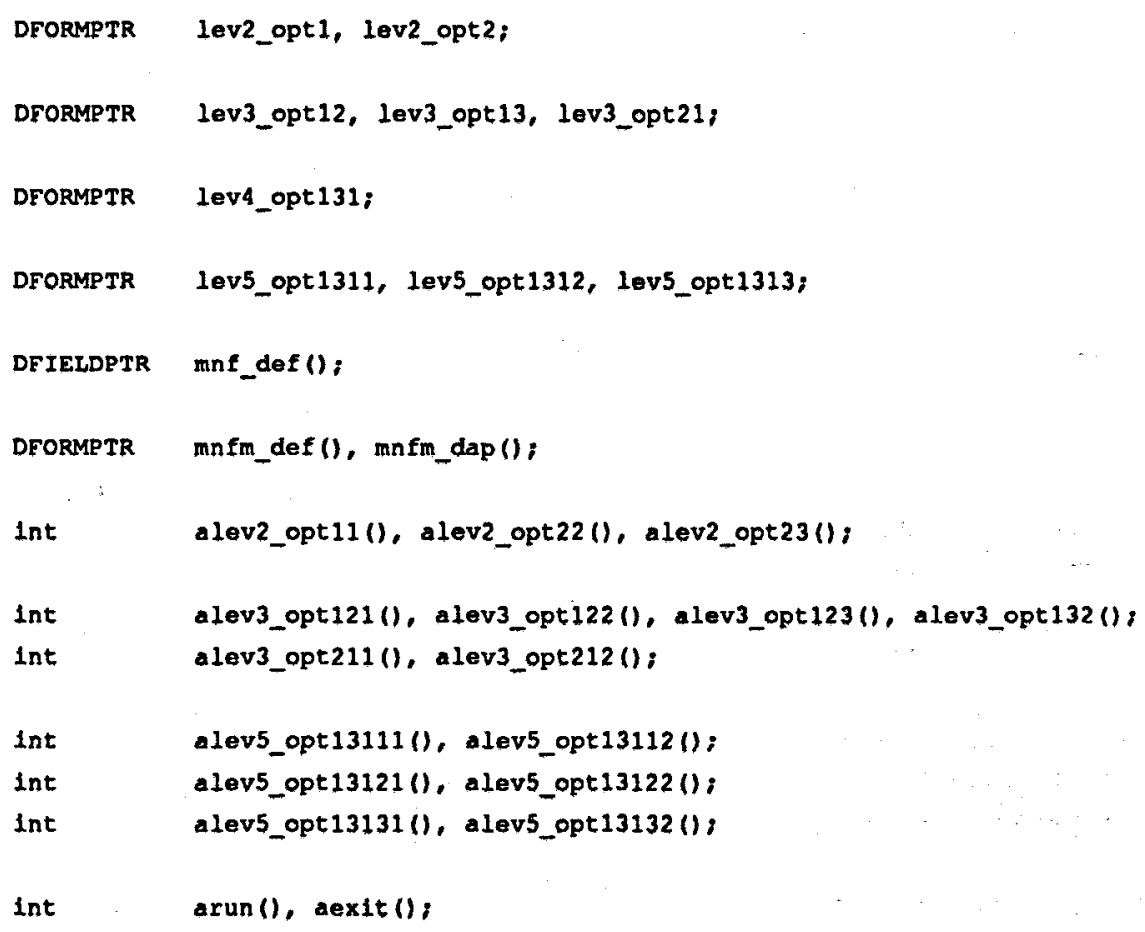




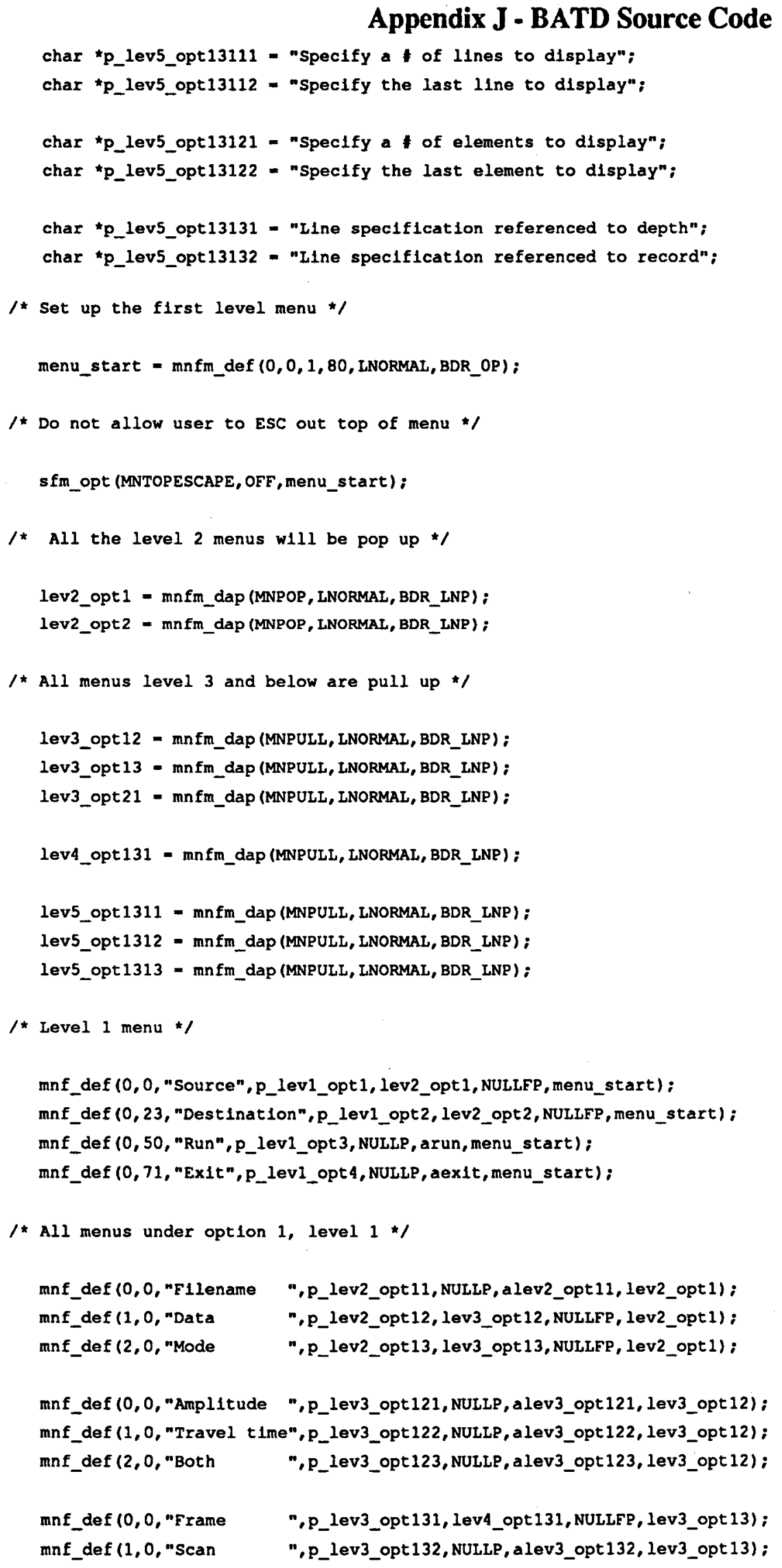




\section{Appendix J - BATD Source Code}

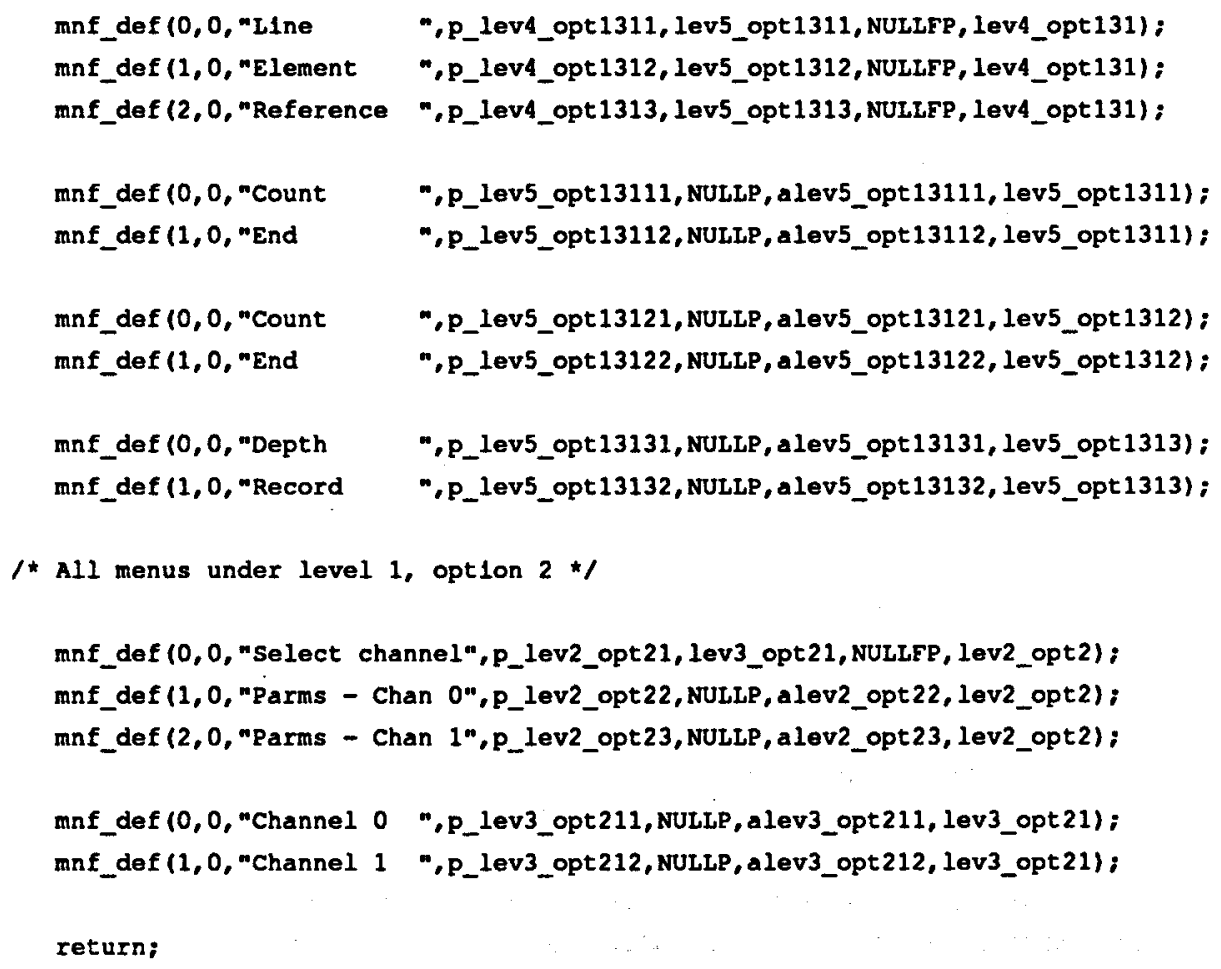




\section{Appendix J - BATD Source Code}

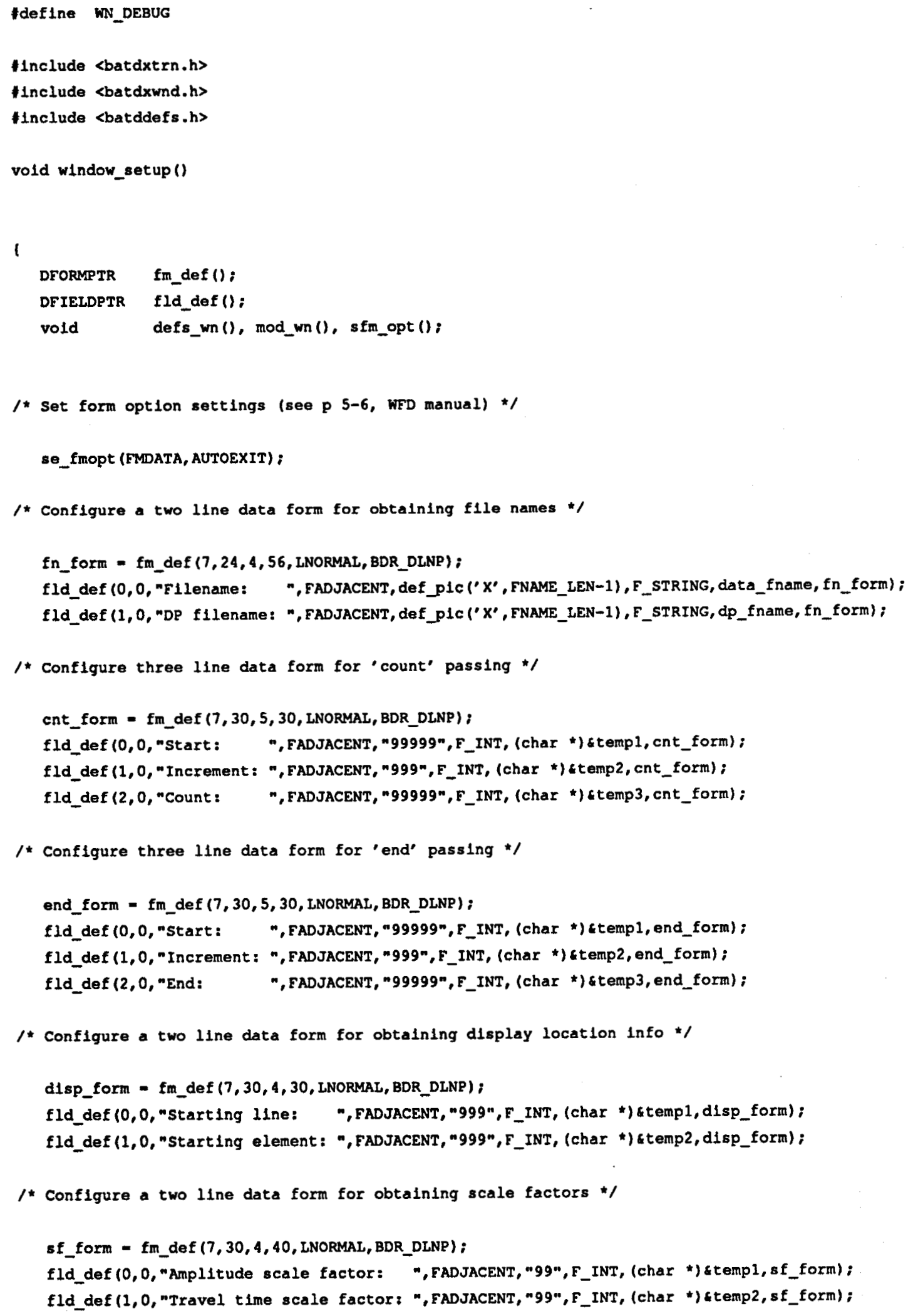




\section{Appendix J - BATD Source Code}

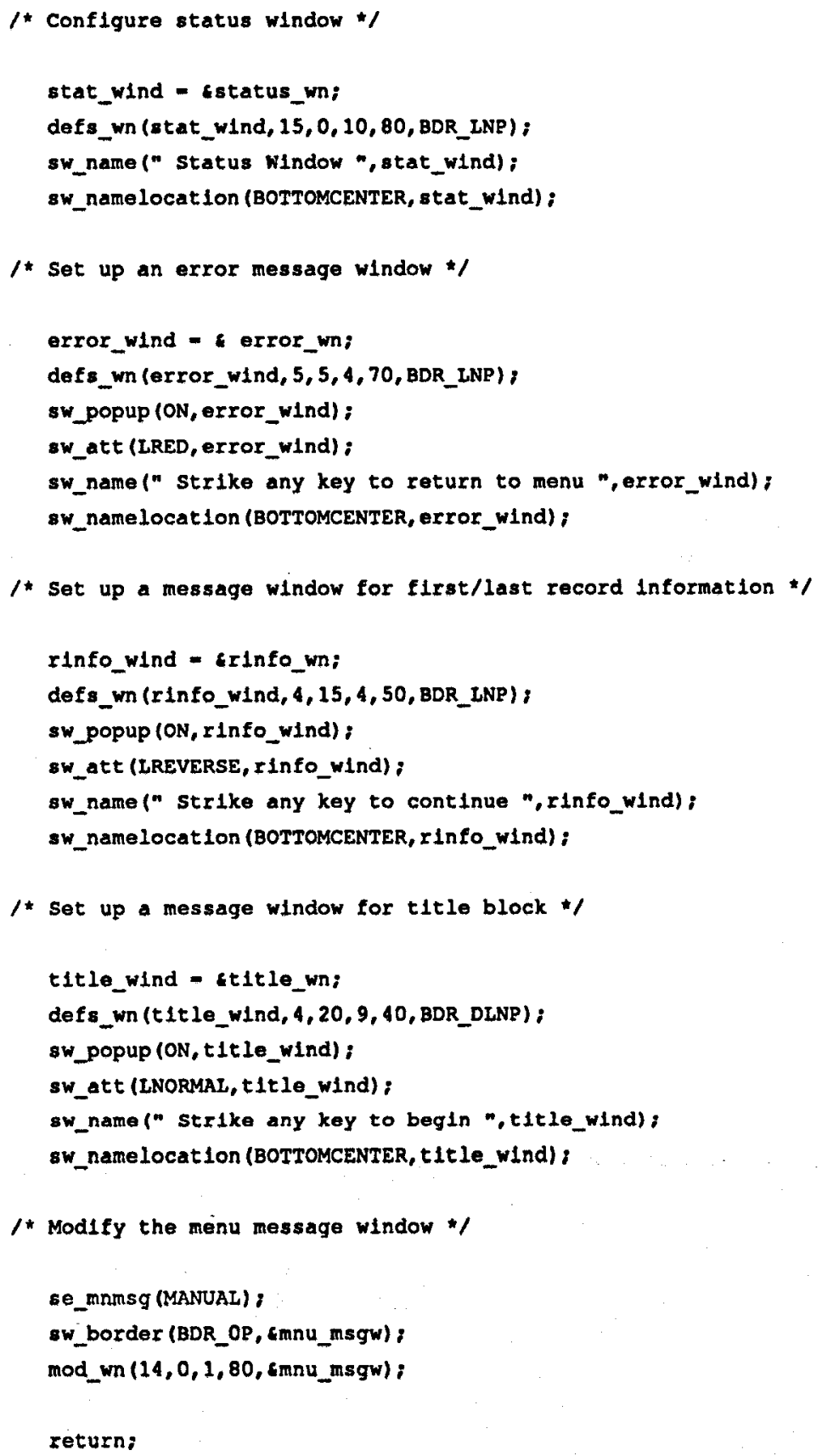




\section{Appendix J - BATD Source Code}

$*$

* DEVELOPED BY: Troy K. Moore

* DATE: $\quad 10 / 7 / 87$

$\star$

* REVISION NOTES:

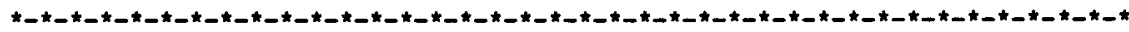

*/

vold put_title()

1

Int temp;

char "IInel = " BAT Tool Data Display Utility\n";

char *line2 = " Developed by Troy $K$. Moore $\backslash n \backslash n "$;

char *11ne3 = " Version: $45 / \mathrm{n}^{n}$;

char *line4 - " Dated: $\$ 8 \mathrm{~s}^{n}$;

/* Prepare for writing to the title window */

set_wn (title_wind):

mv_cs $(1,0$, title_wind $)$;

1 * Place information in the window */

v_st (linel,title_wind);

v_st (line2,t1tle_wind):

v_printf(title_wind, line3,vER);

v_printf(title_wind, line4,VER_DATE);

/* Walt for input from the keyboard before removing window and beginning */

temp $=k 1()$;

unset_wn (title_wind);

return;

1

$1 *$

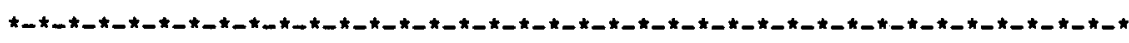

$\cdot$

* MODULE NAME: fill_stat_wn

-

- DESCRIPTION: This function is designed to flll the status window

- with initial parameter information at the start of code execution.

-

- NoTES: f111_stat_wn is called directly by batd.c

102 


\section{Appendix J - BATD Source Code}

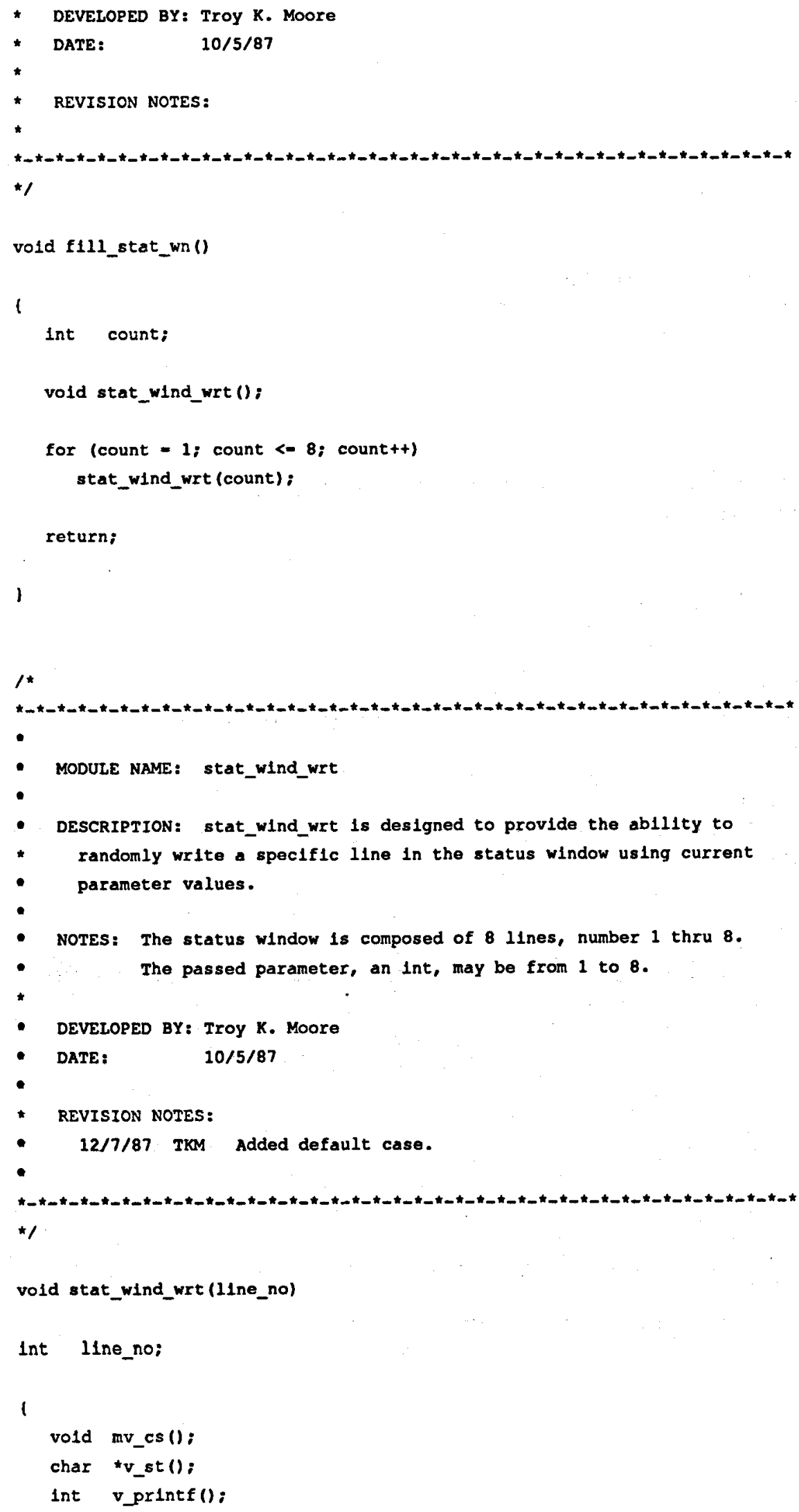




\section{Appendix J - BATD Source Code}

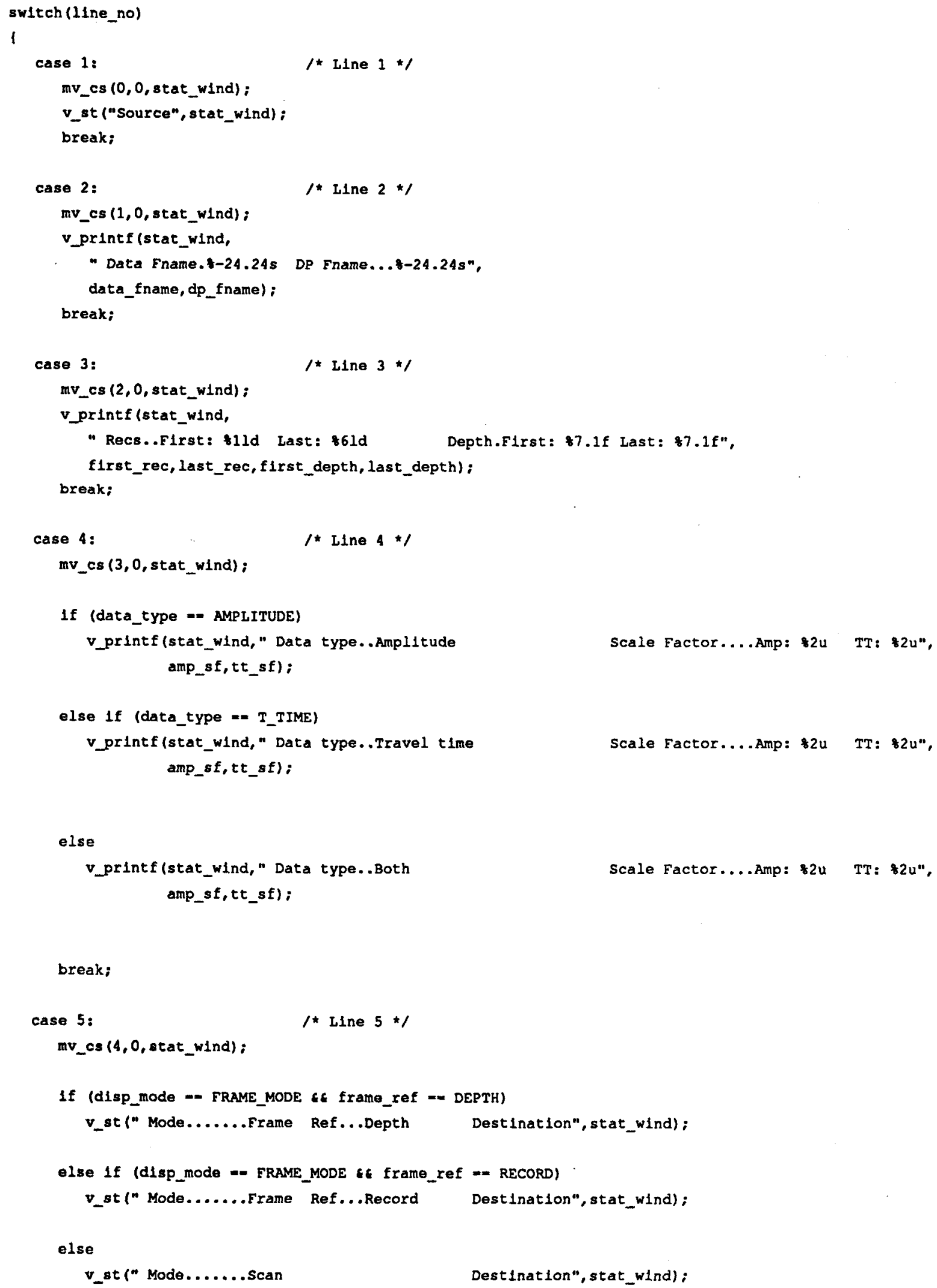




\section{Appendix J - BATD Source Code}

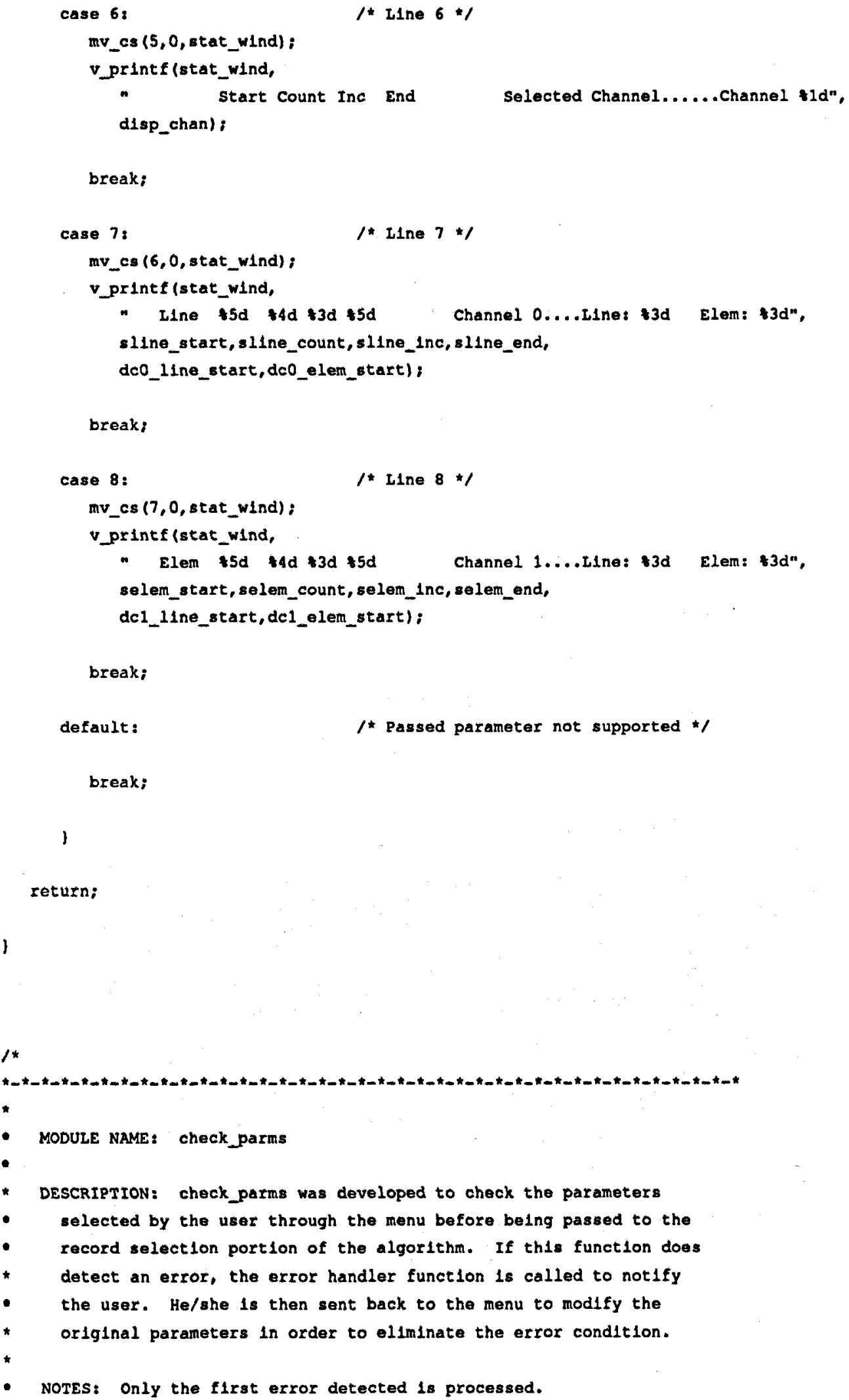




\section{Appendix J - BATD Source Code}

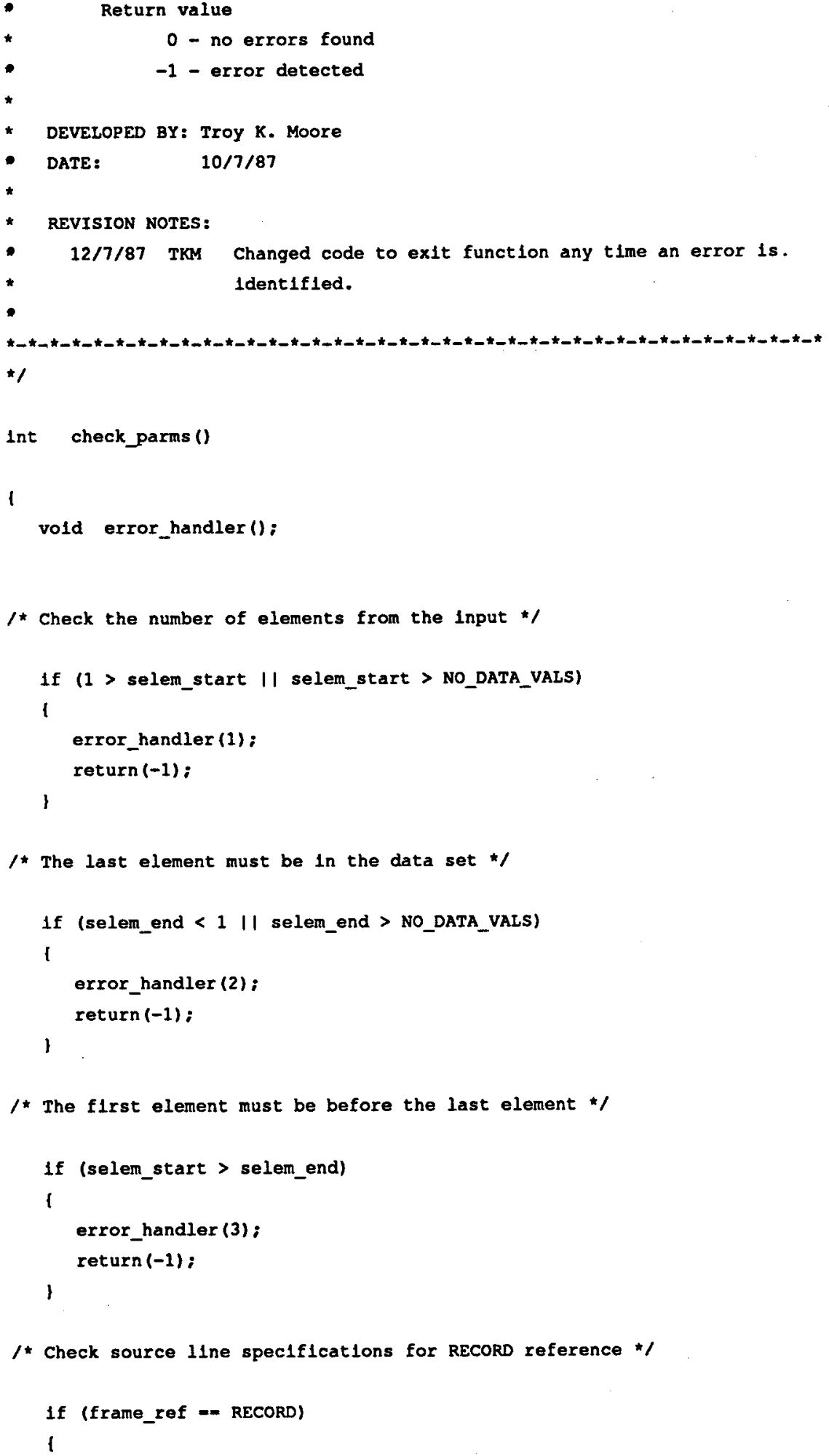




\section{Appendix J - BATD Source Code}

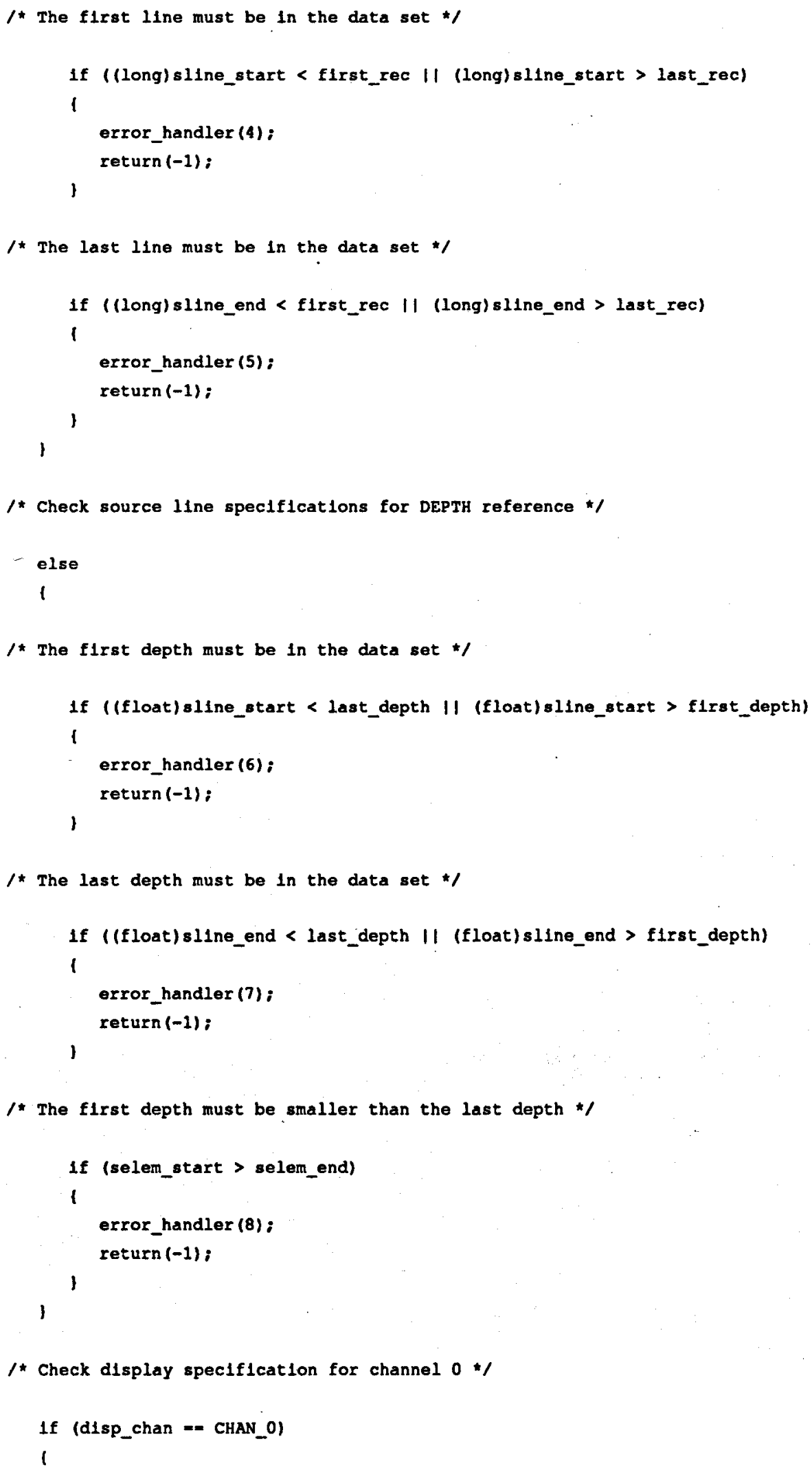




\section{Appendix J - BATD Source Code}

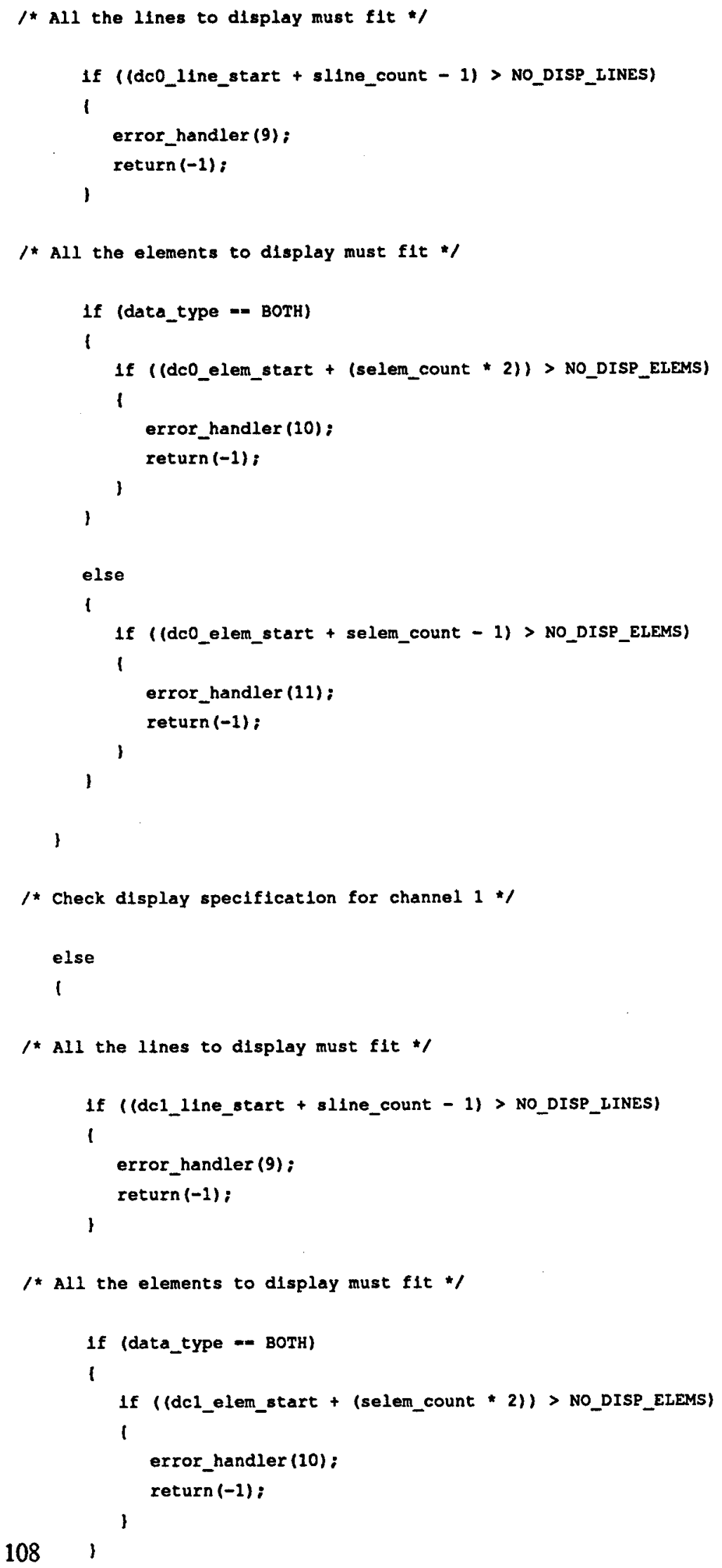




\section{Appendix J - BATD Source Code}

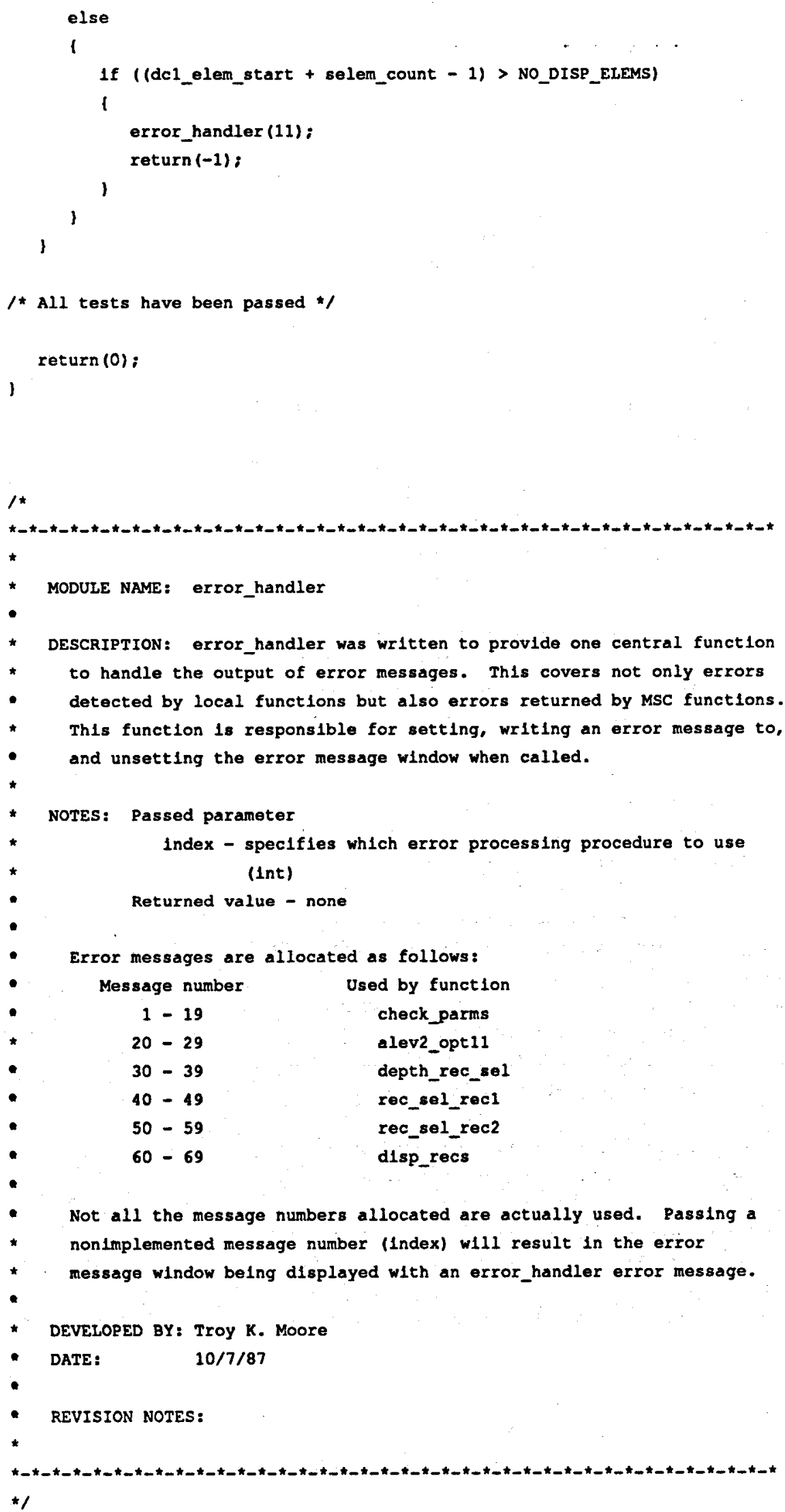




\section{Appendix J - BATD Source Code}

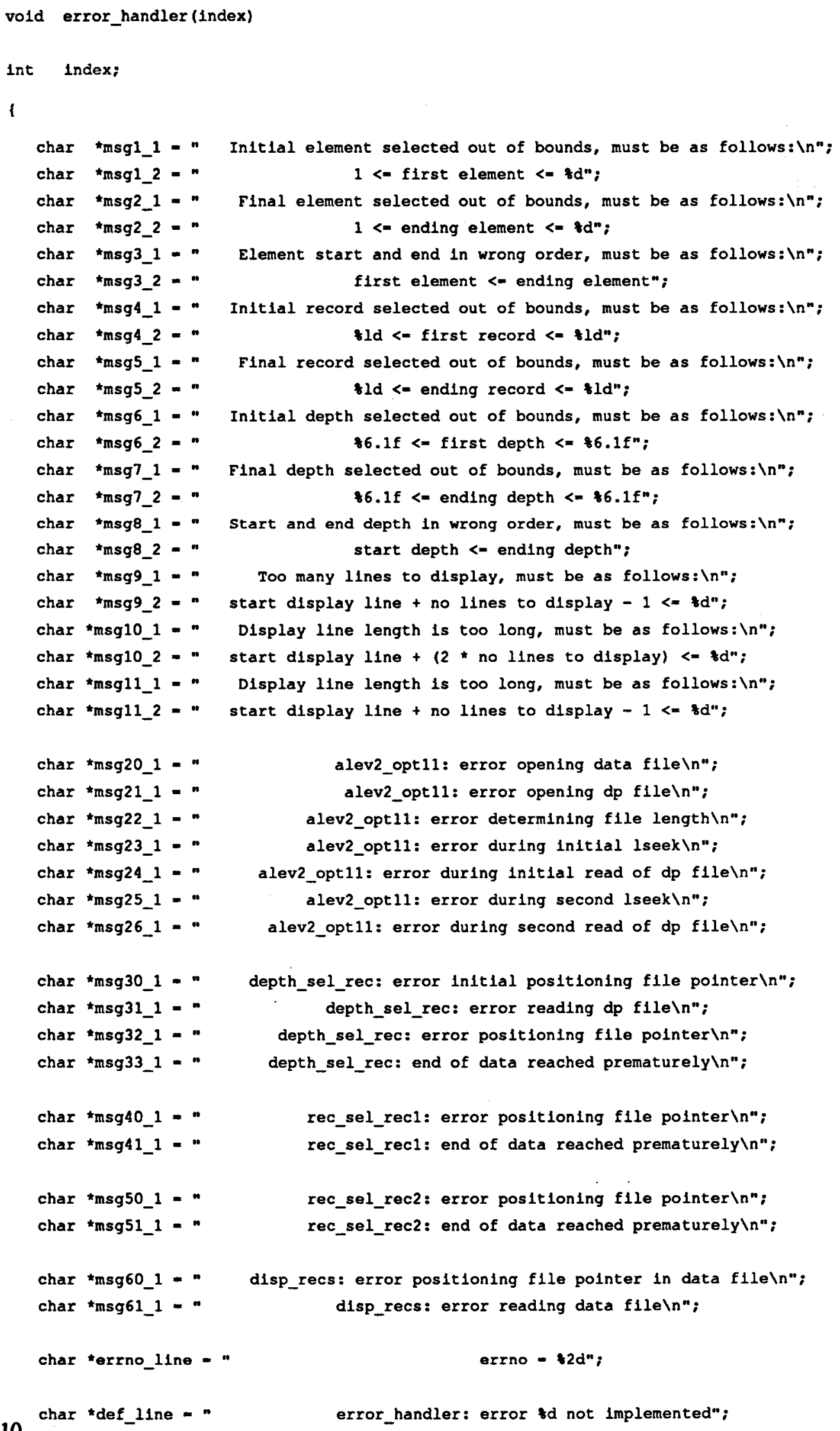




\section{Appendix J - BATD Source Code}

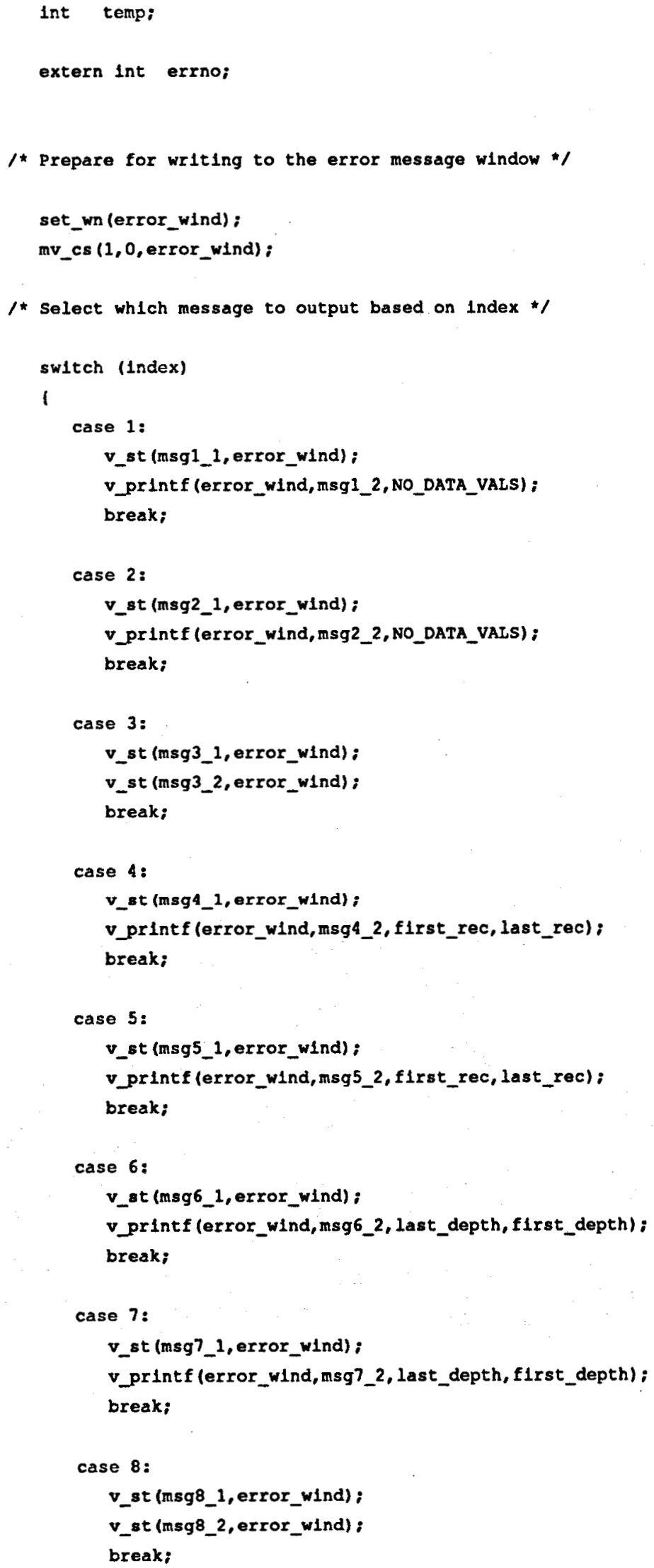




\section{Appendix J - BATD Source Code}

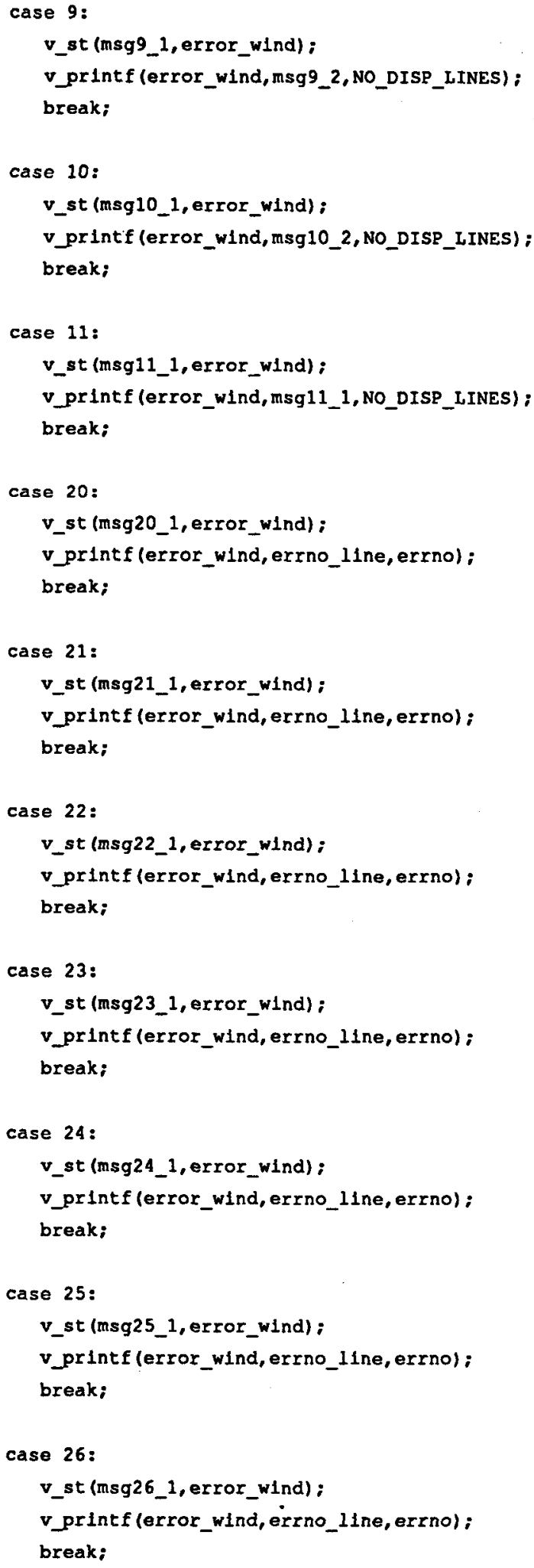




\section{Appendix J - BATD Source Code}

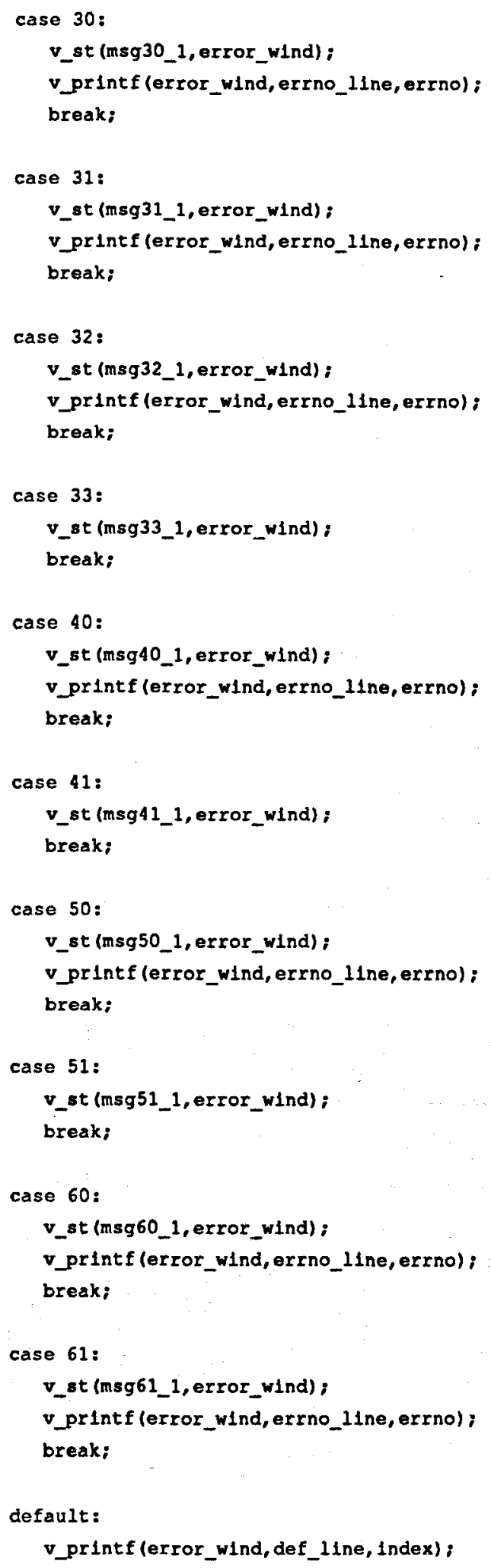




\section{Appendix J - BATD Source Code}

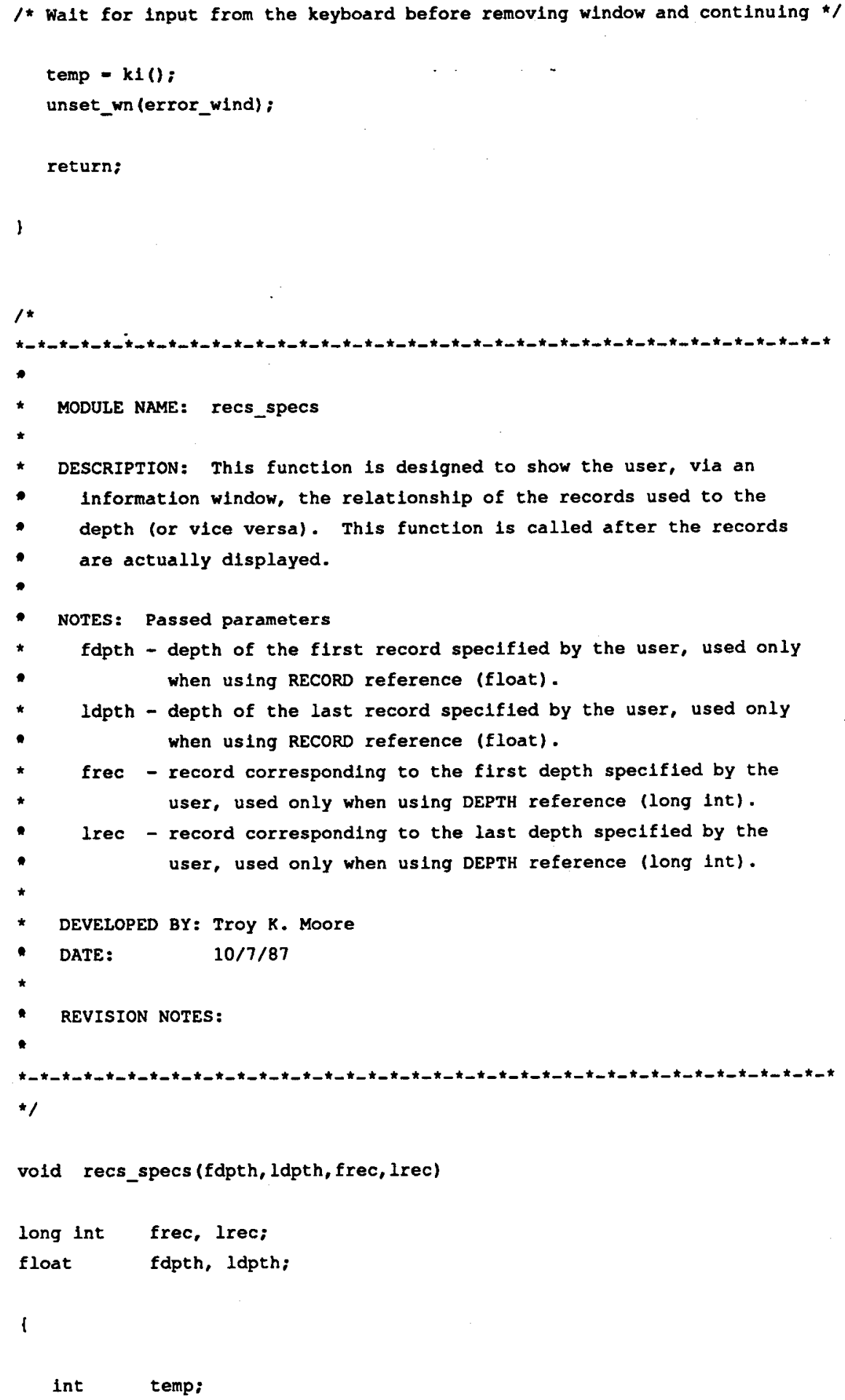




\section{Appendix J - BATD Source Code}

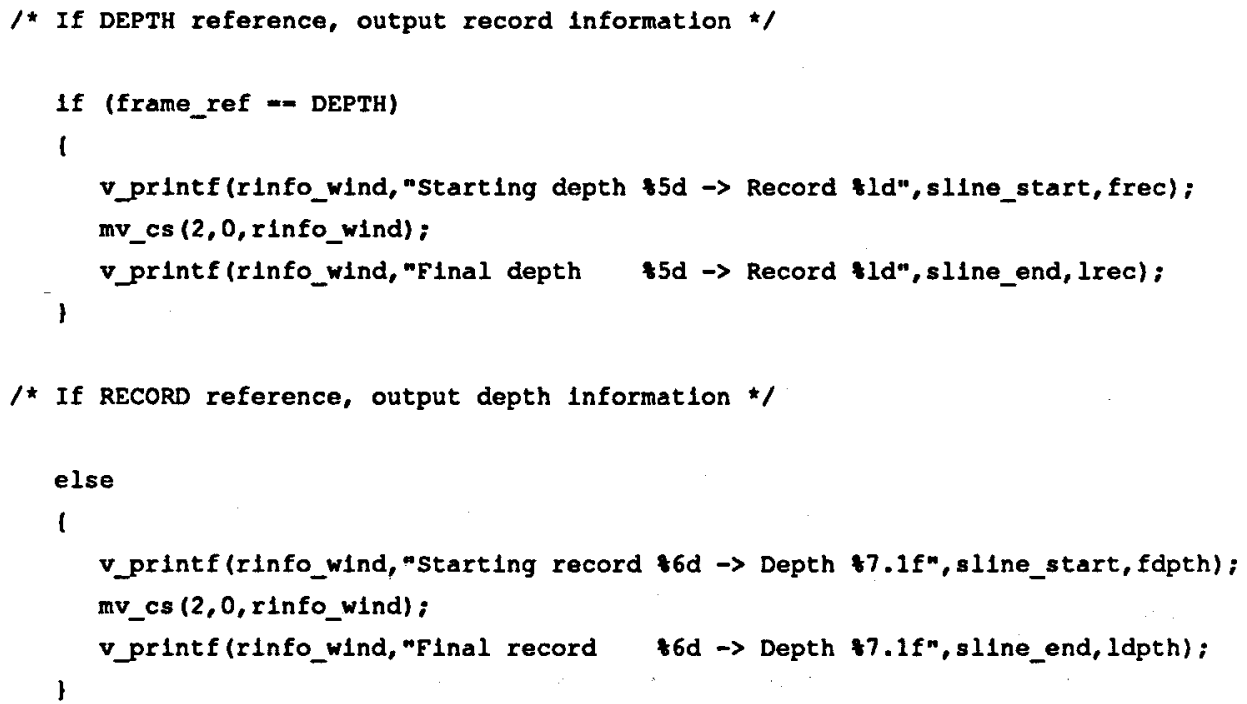




\section{Appendix J - BATD Source Code}

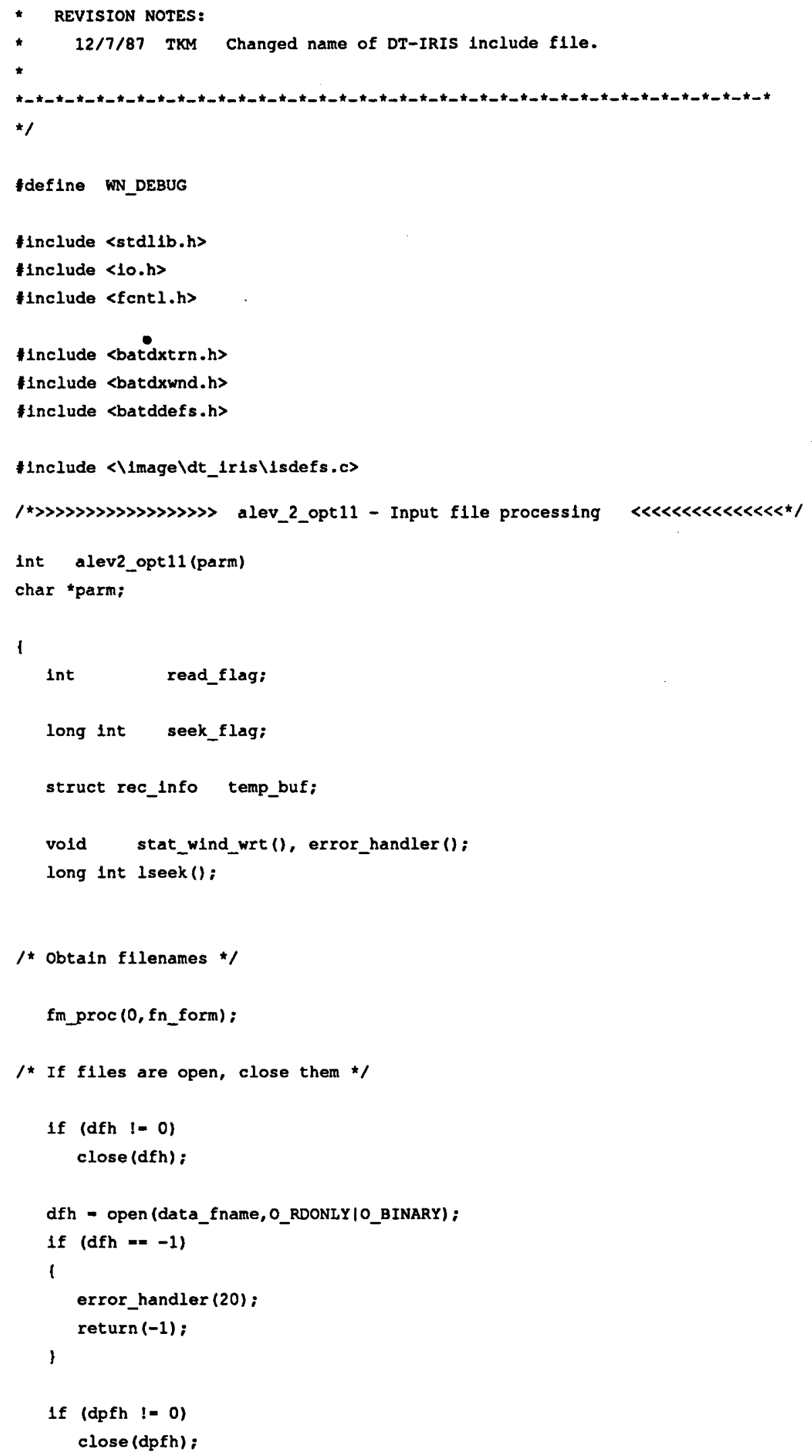




\section{Appendix J - BATD Source Code}

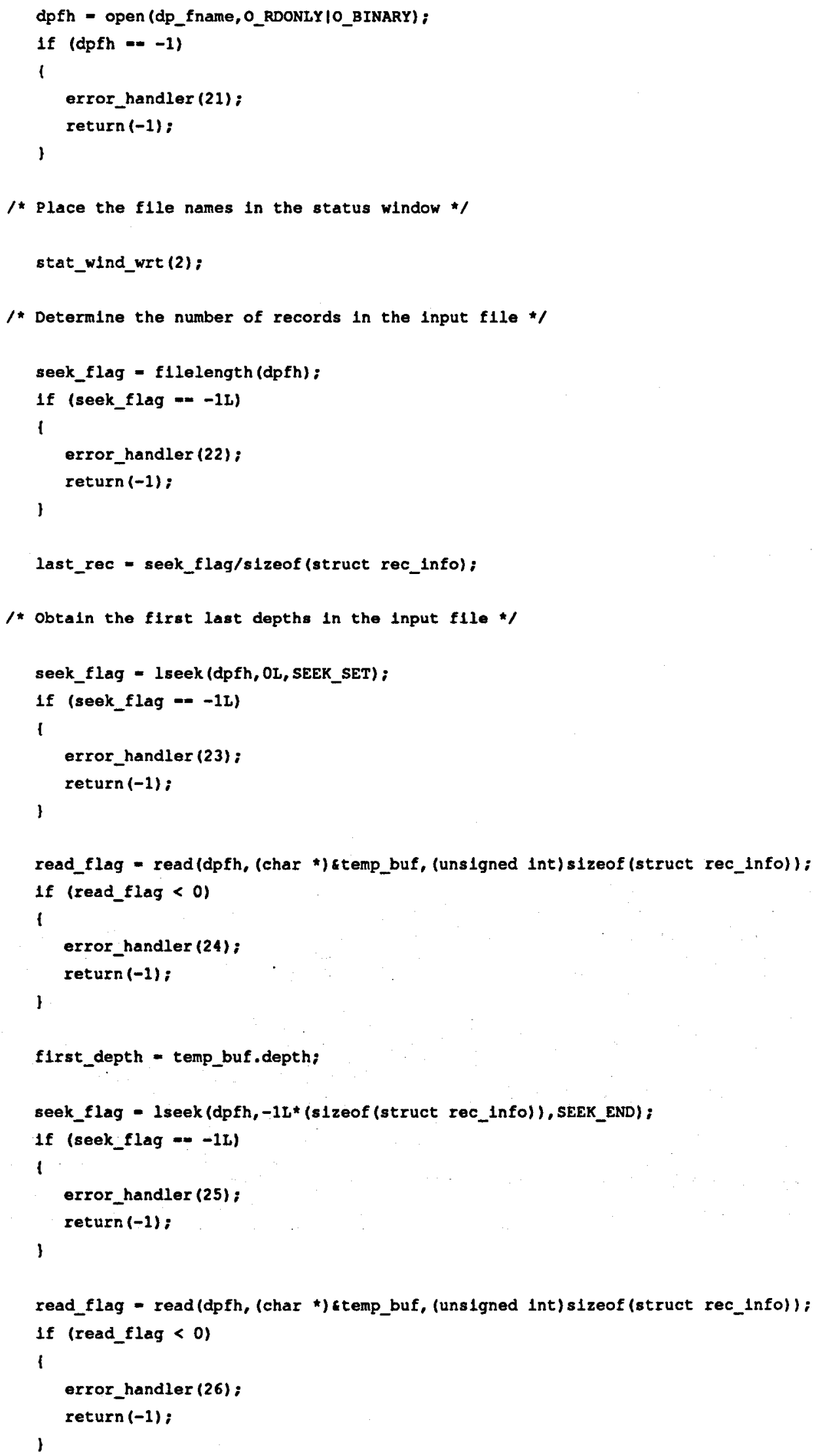




\section{Appendix J - BATD Source Code}

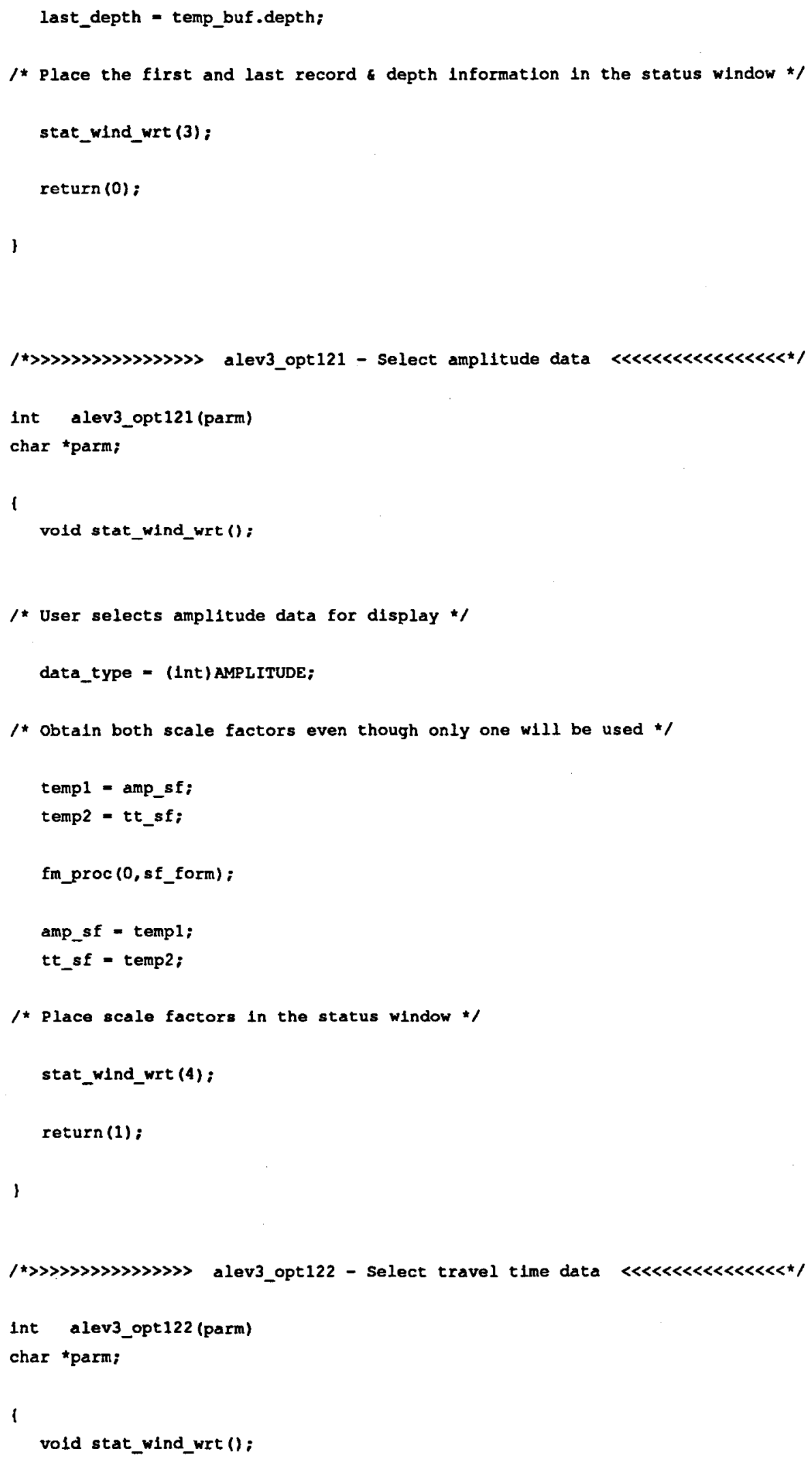




\section{Appendix J - BATD Source Code}

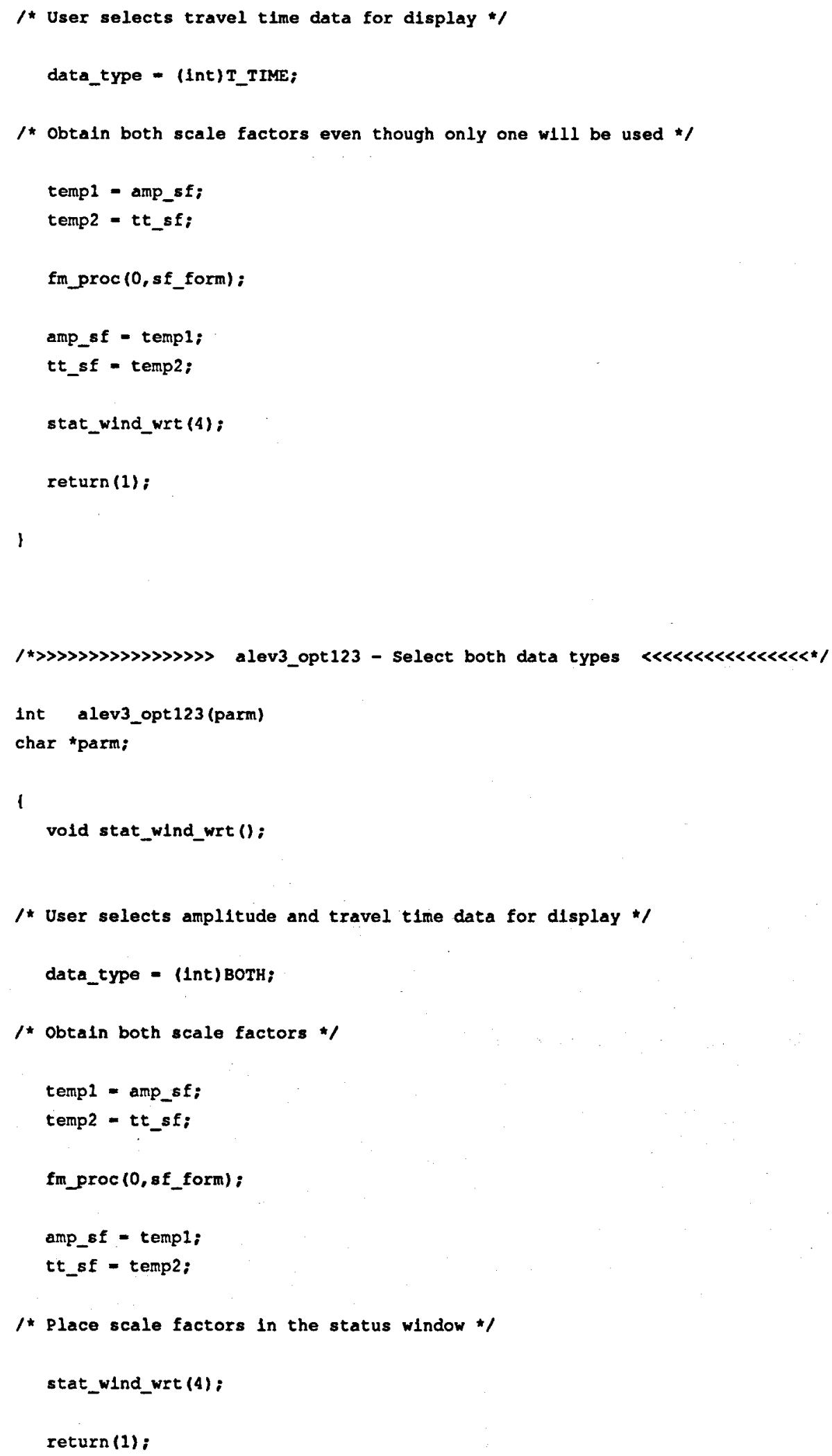




\section{Appendix J - BATD Source Code}

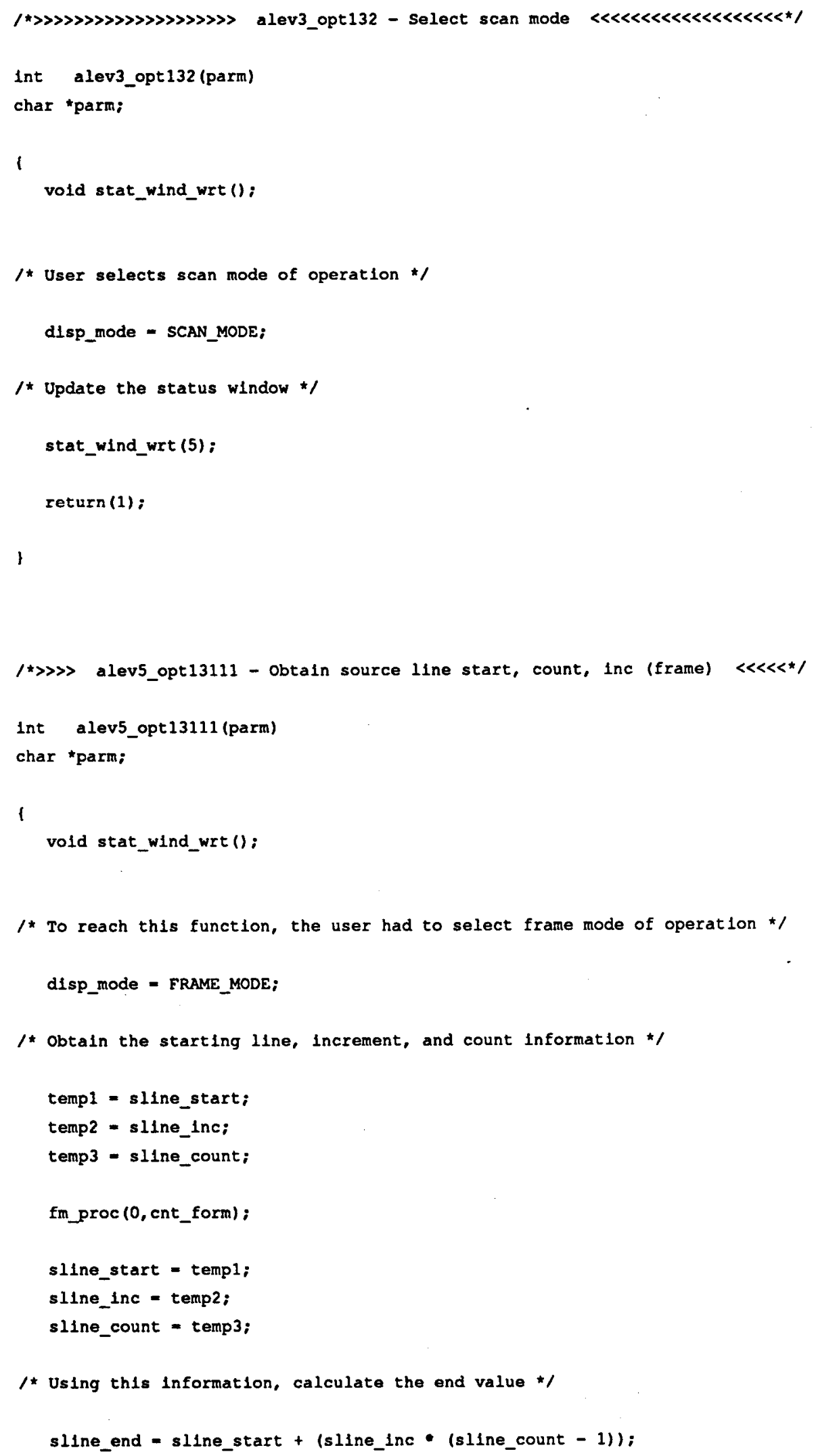




\section{Appendix J - BATD Source Code}

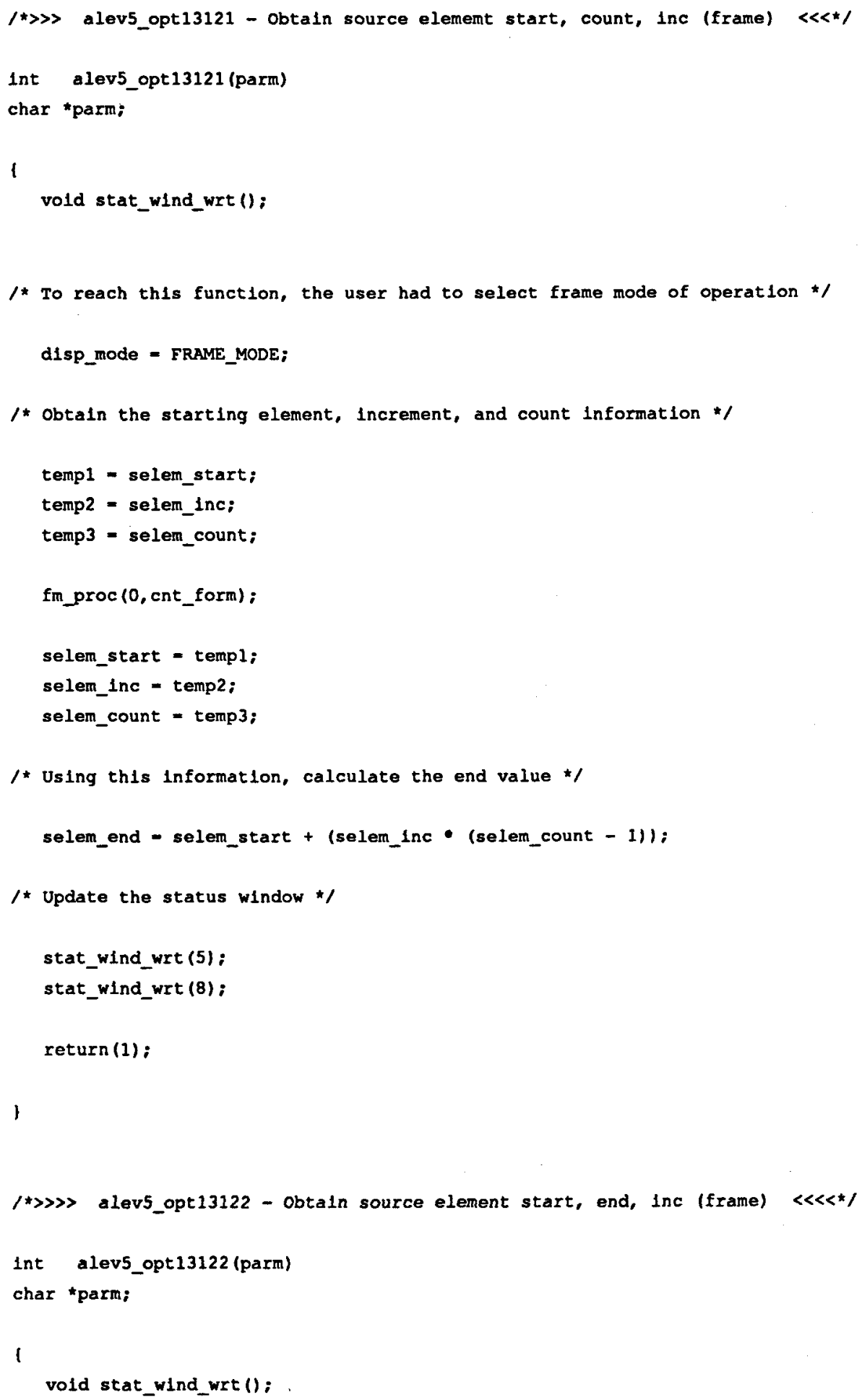




\section{Appendix J - BATD Source Code}

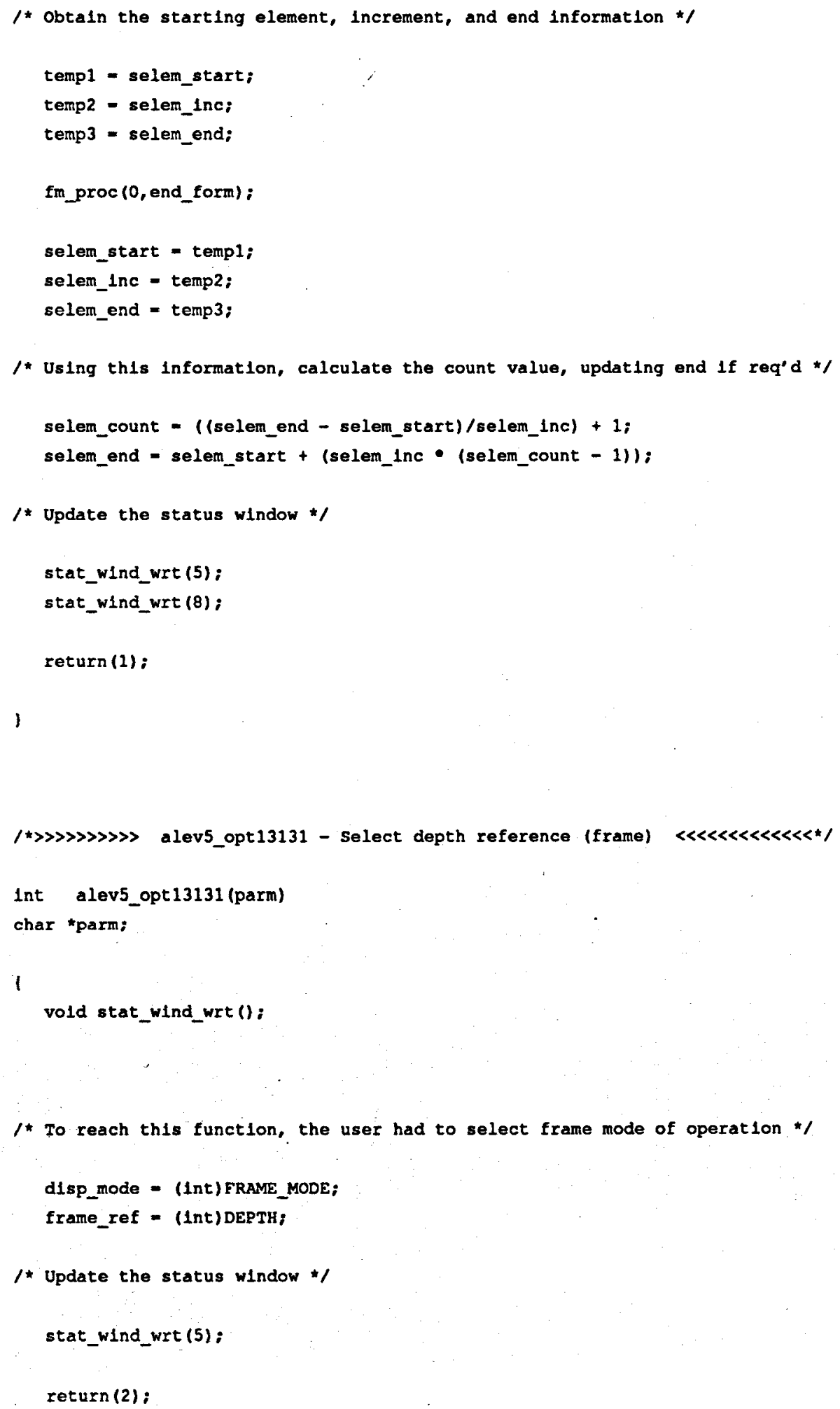




\section{Appendix J - BATD Source Code}

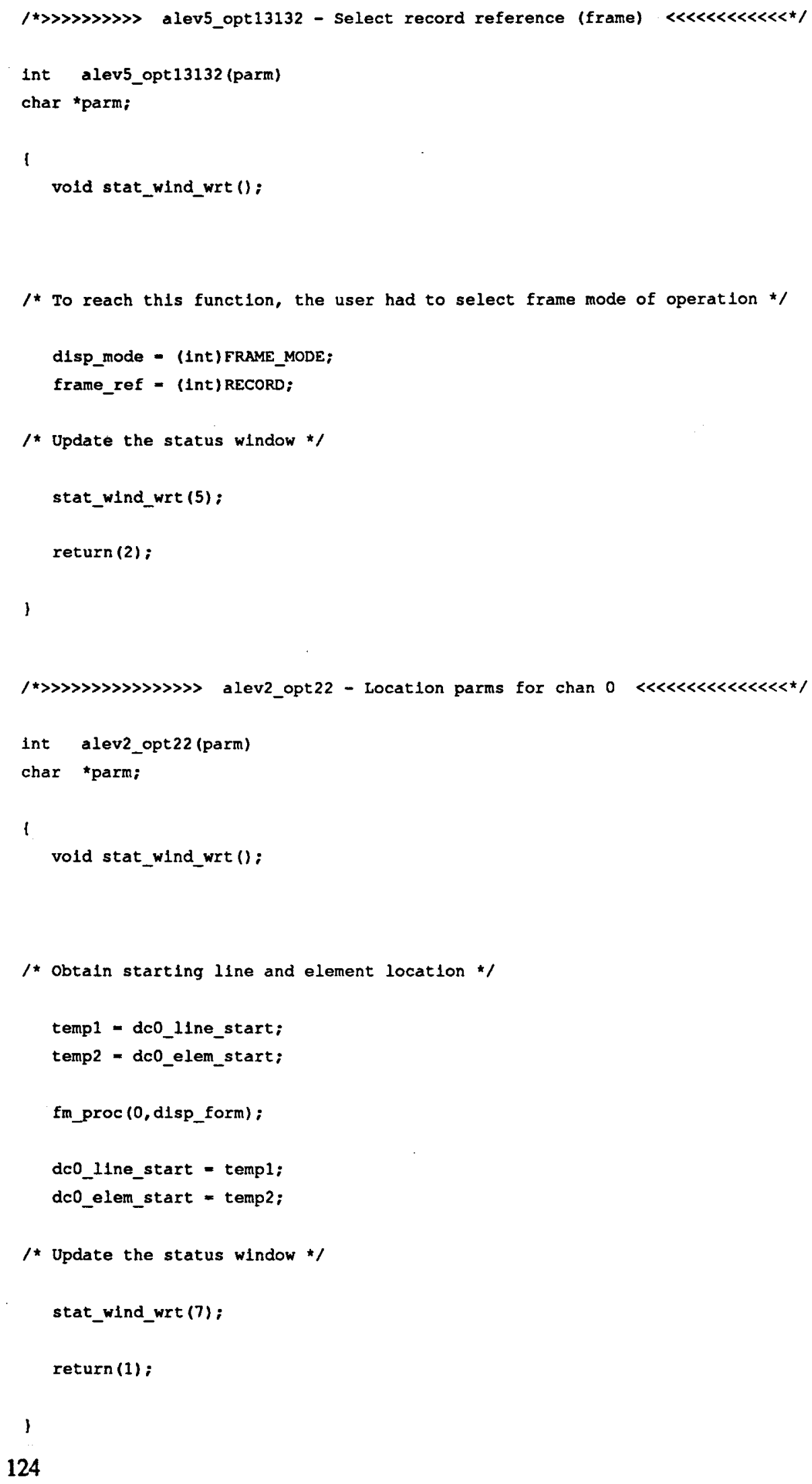




\section{Appendix J - BATD Source Code}

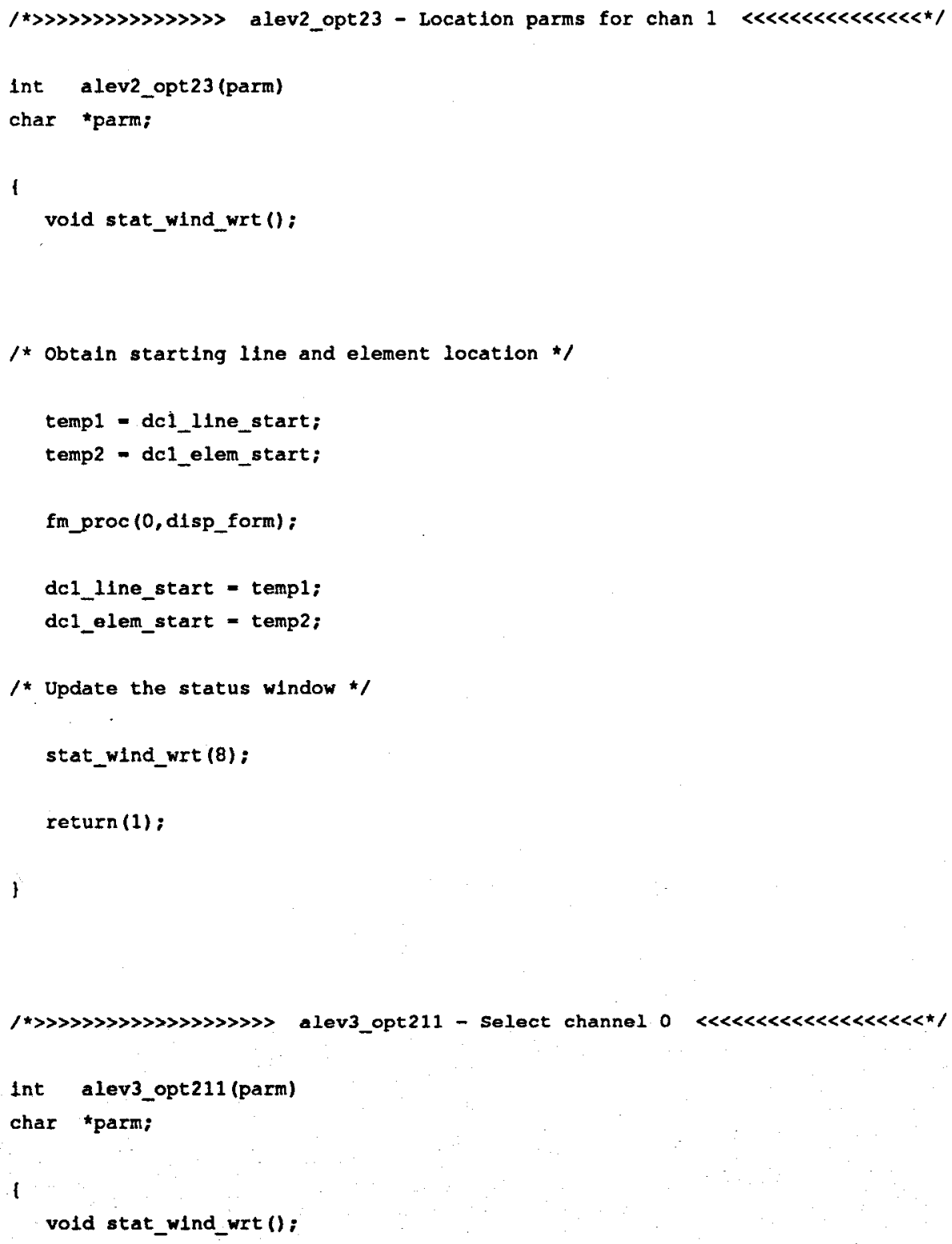




\section{Appendix J - BATD Source Code}

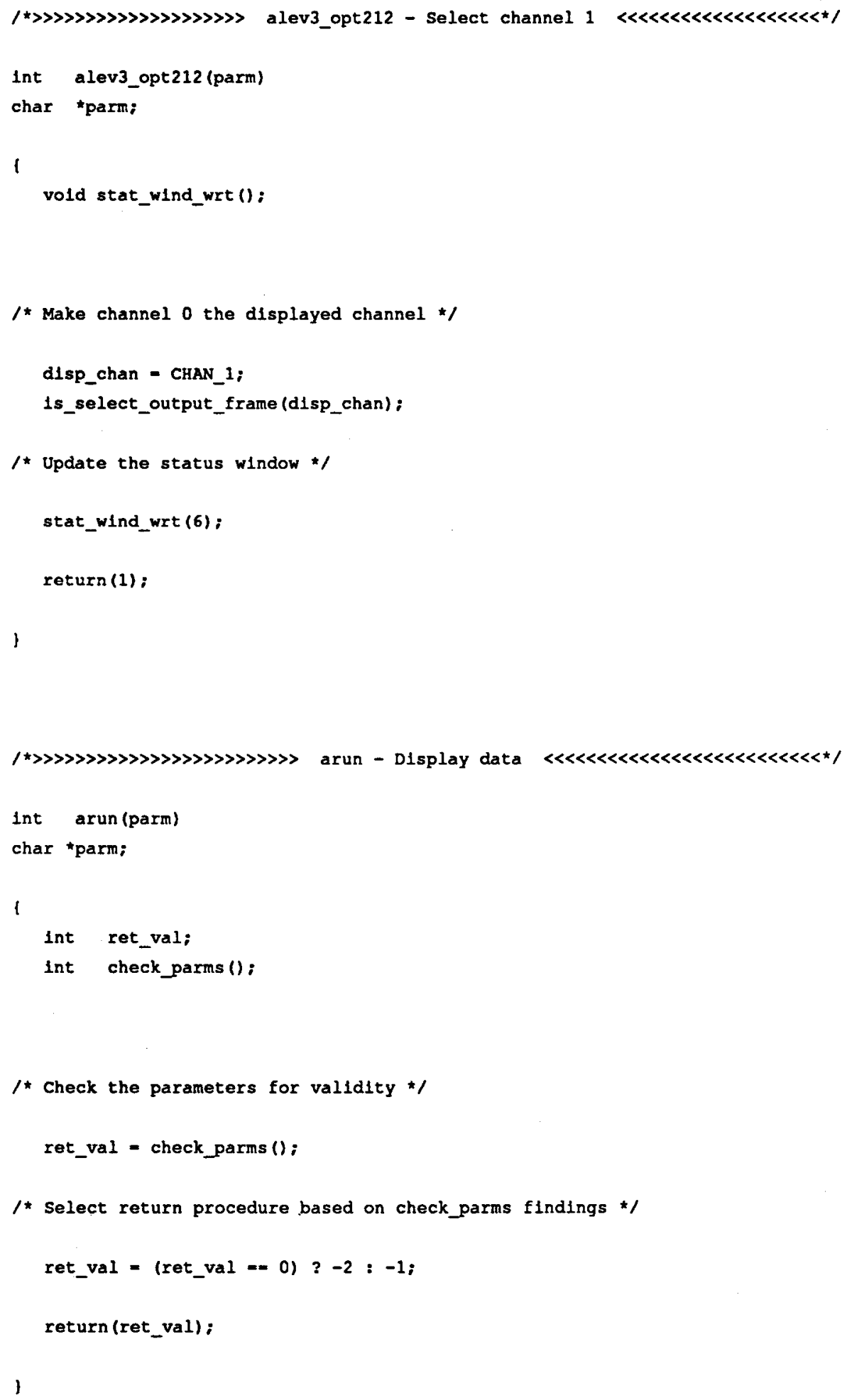




\section{Appendix J - BATD Source Code}

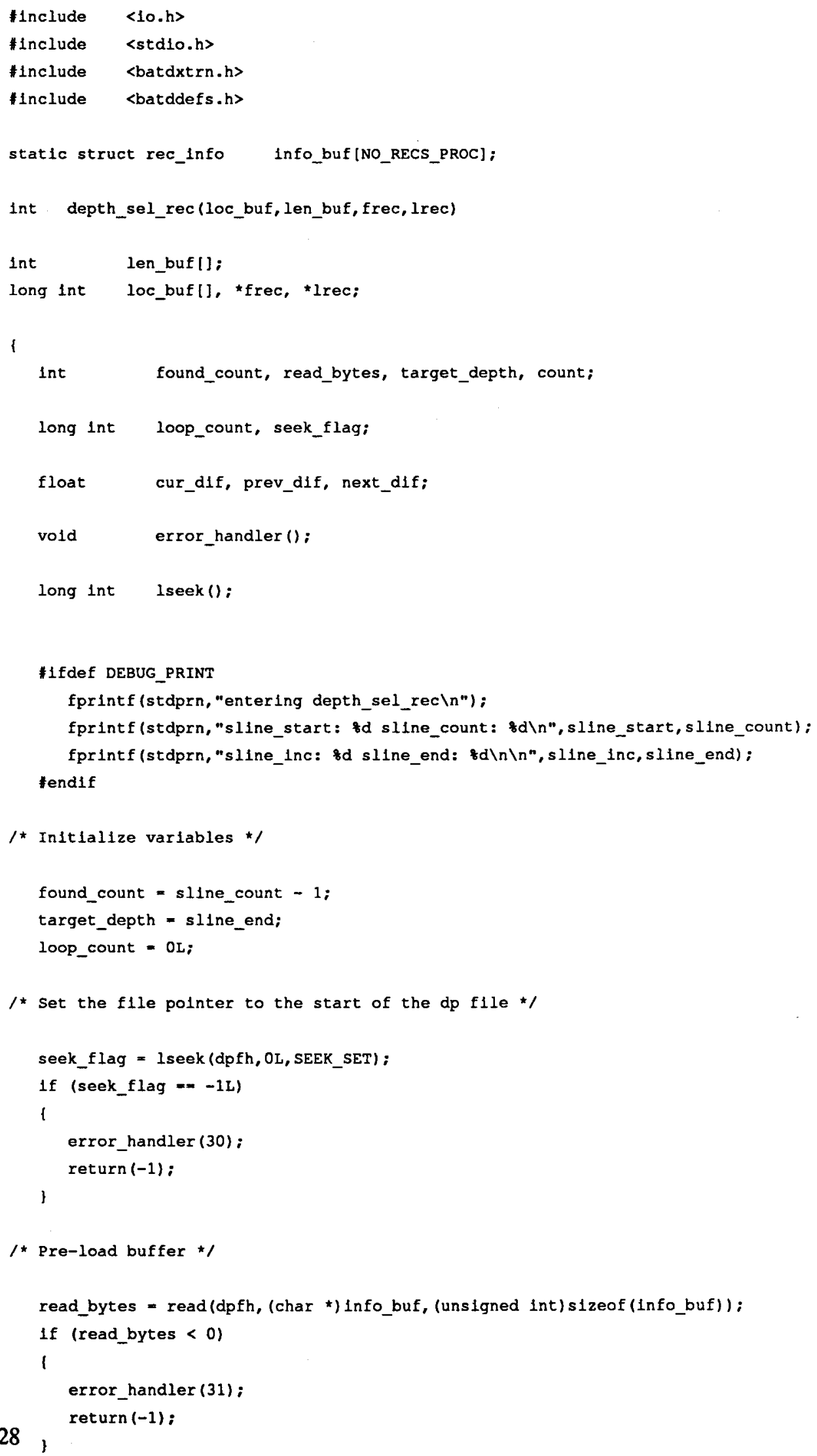




\section{Appendix J - BATD Source Code}

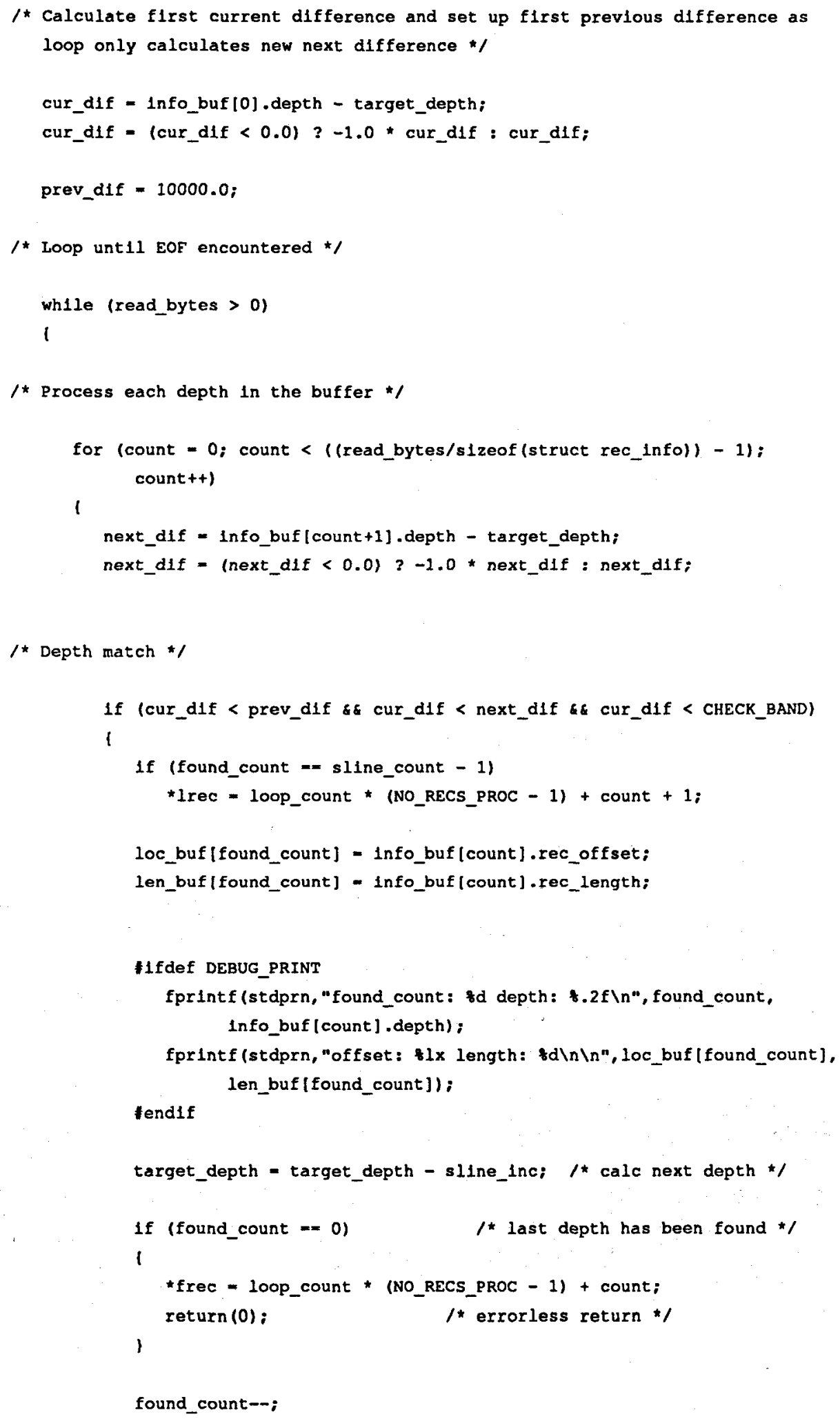




\section{Appendix J - BATD Source Code}

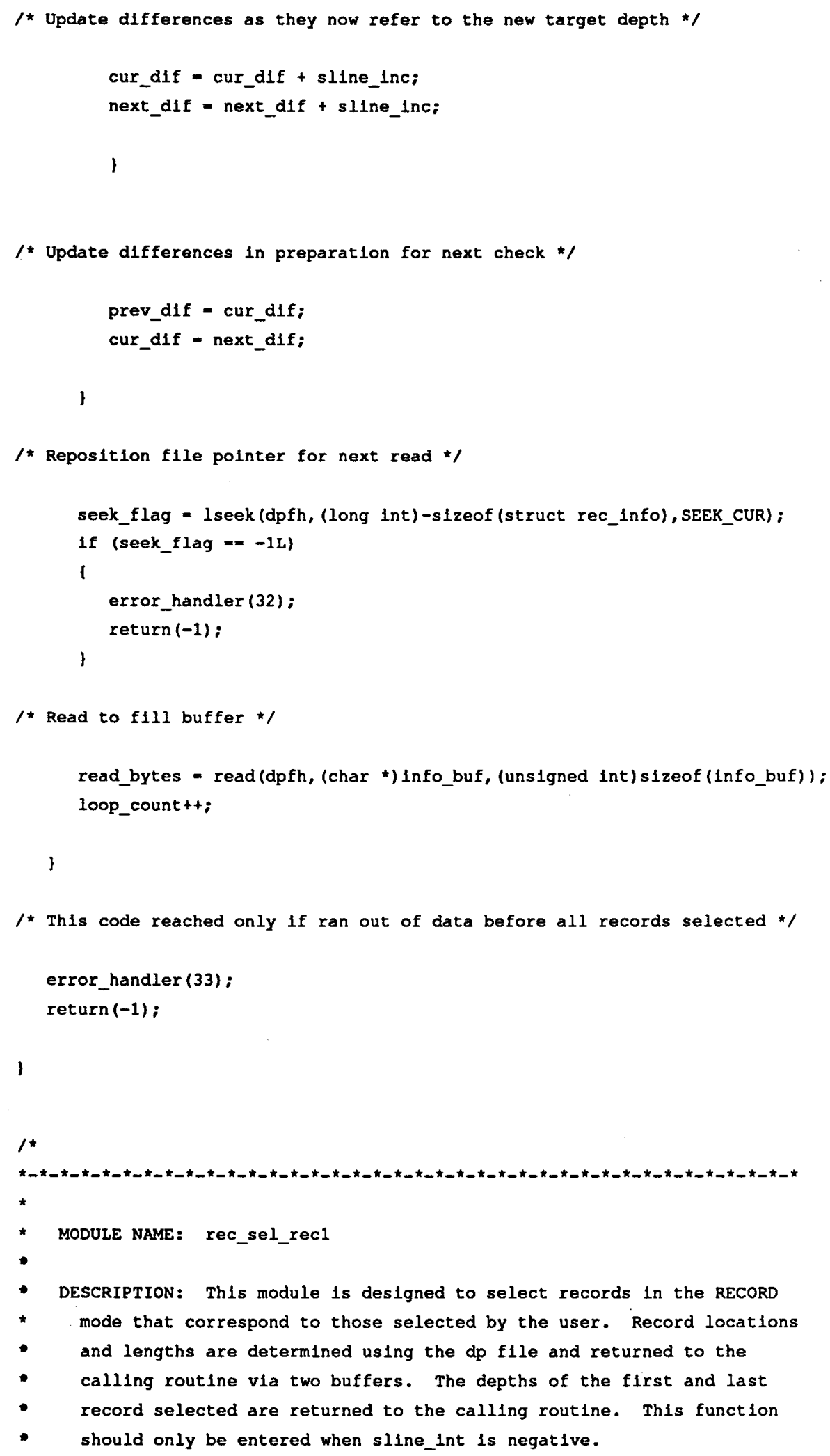




\section{Appendix J - BATD Source Code}

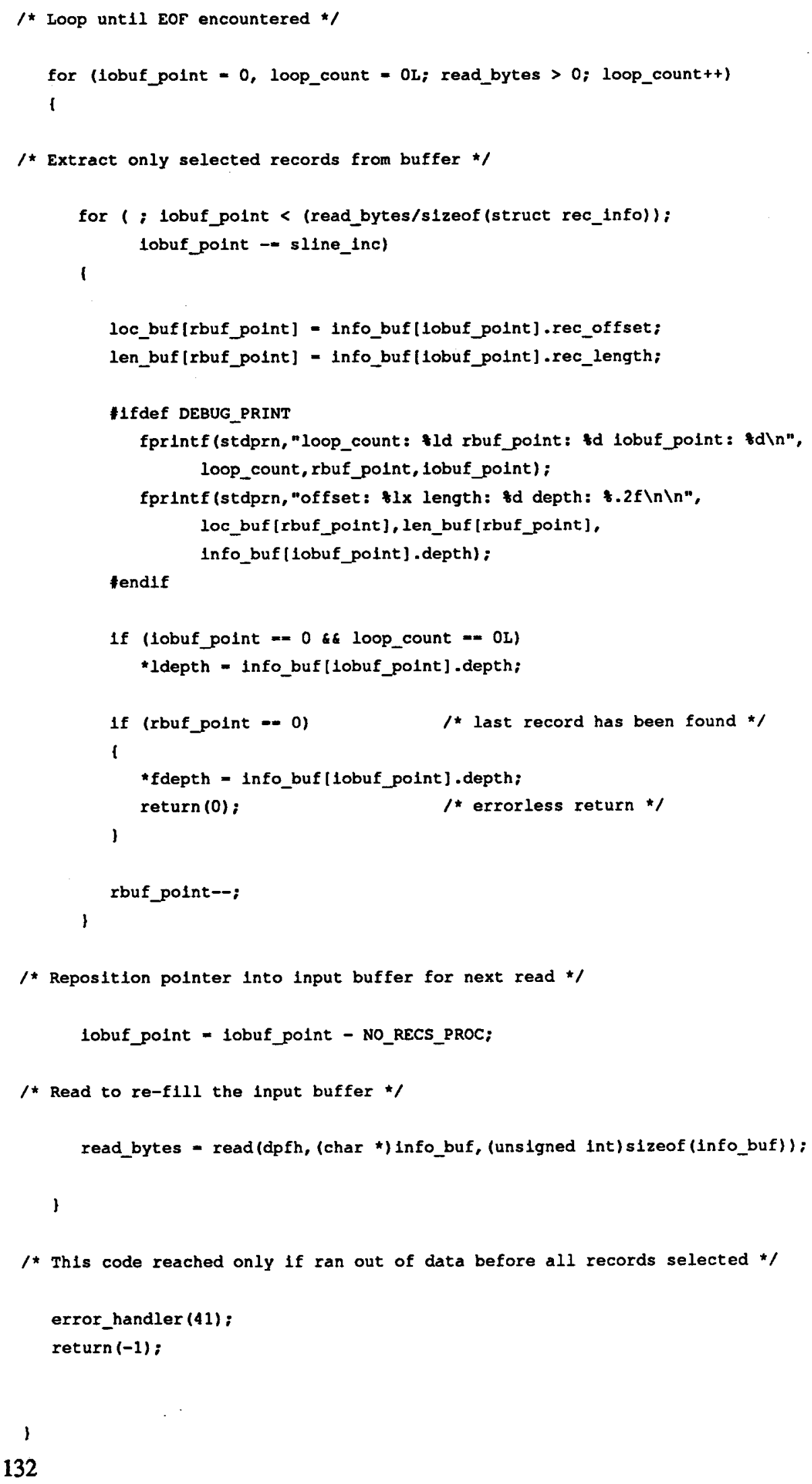




\section{Appendix J - BATD Source Code}

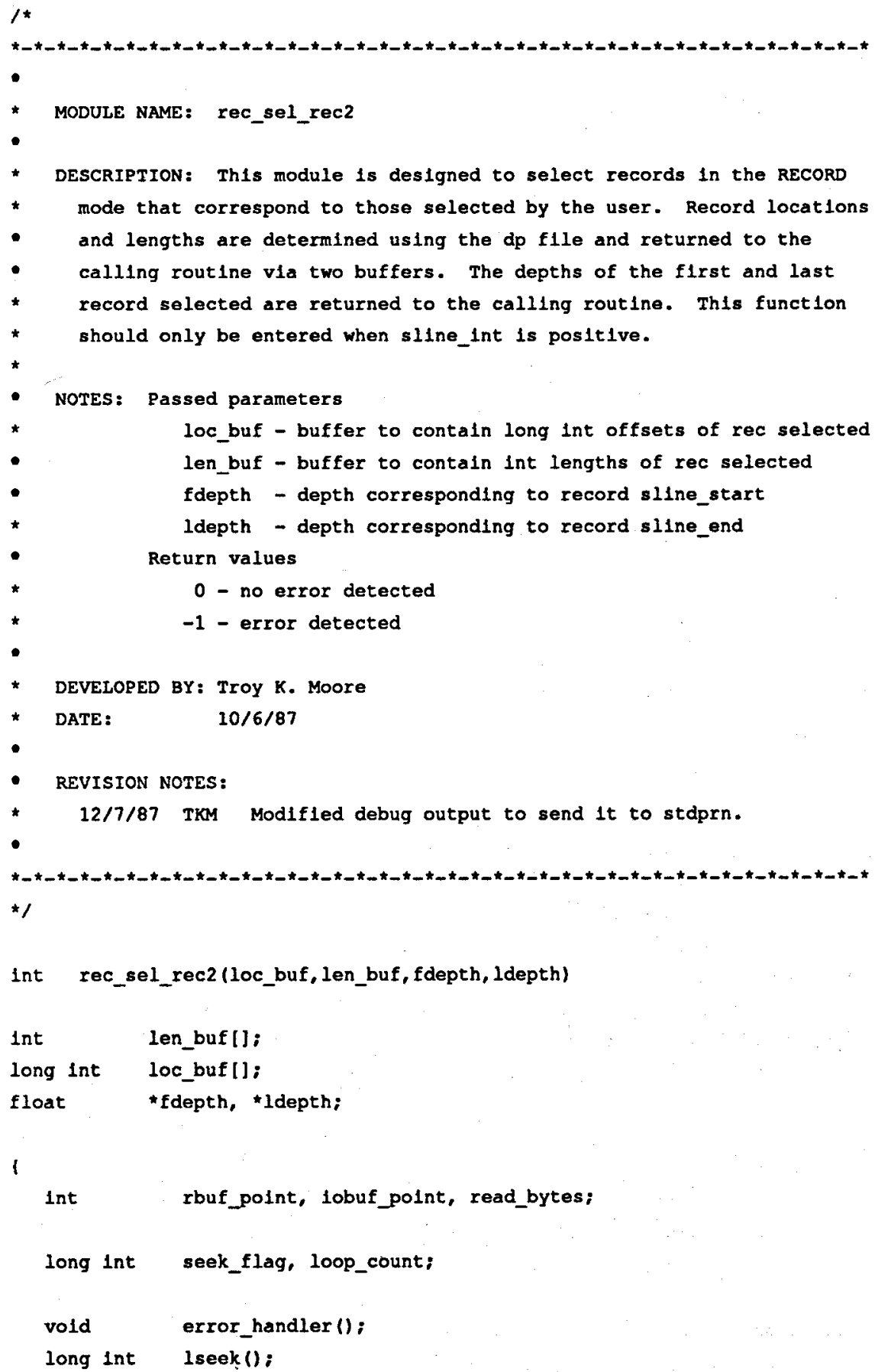

1* Position file pointer to flrst record to process which, in this case, is the record specifled by sline_start */

seek_flag $=1$ seek $($ dpfh, (long int) (s1zeof (struct rec_info)*(sllne_start-1)),SEEK_SET); 


\section{Appendix J - BATD Source Code}

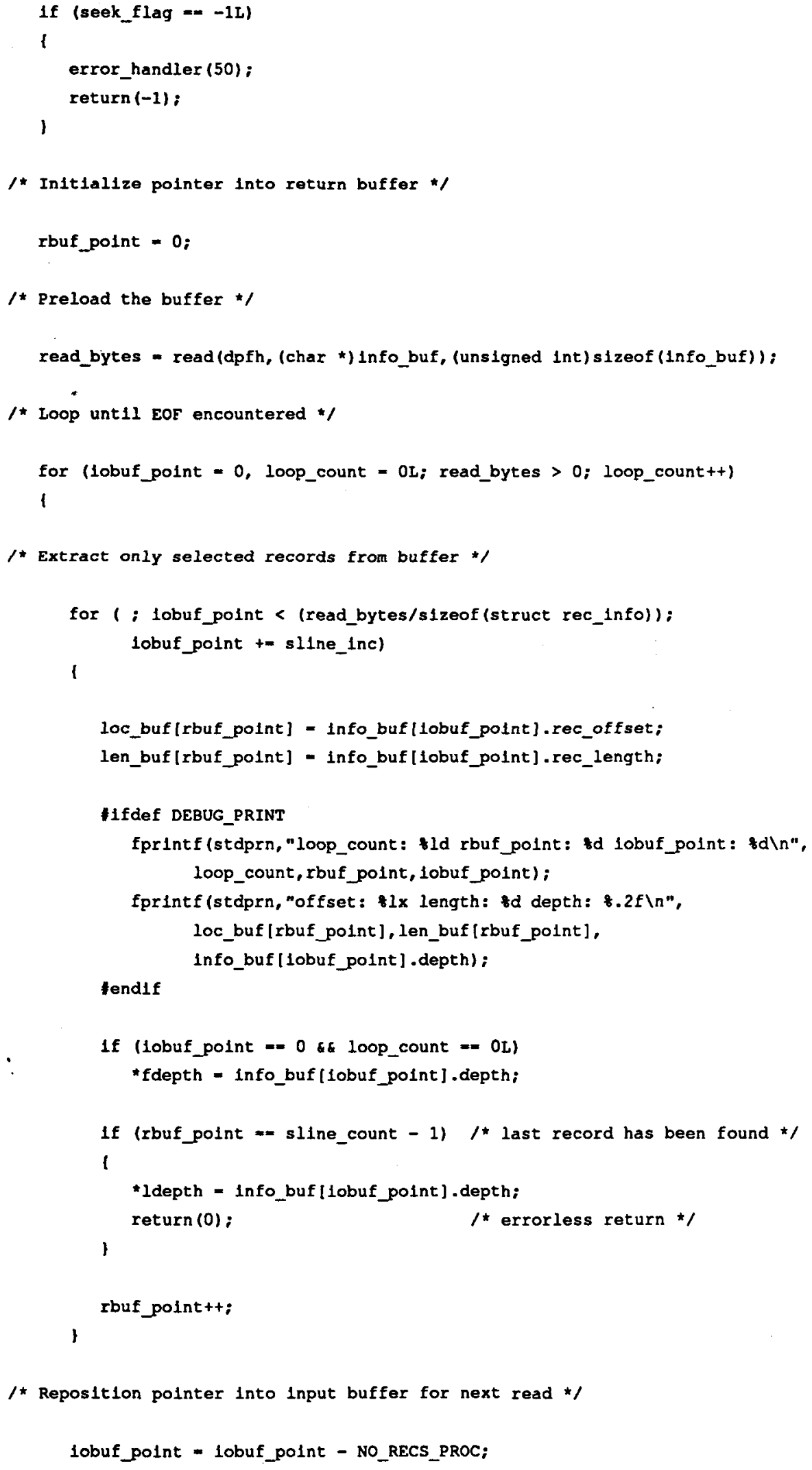




\section{Appendix J - BATD Source Code}

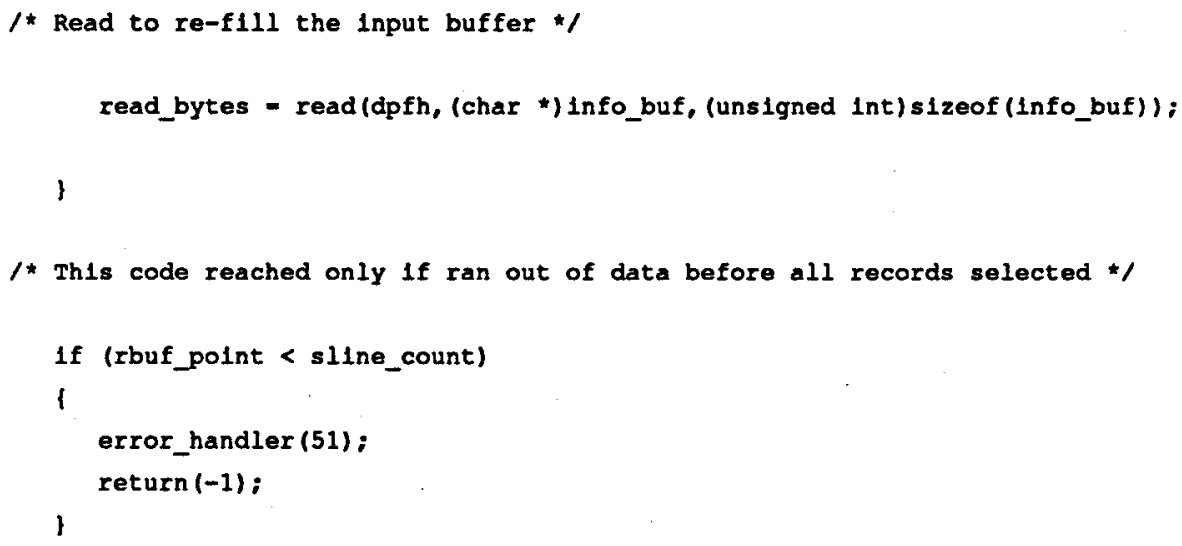




\section{Appendix J - BATD Source Code}

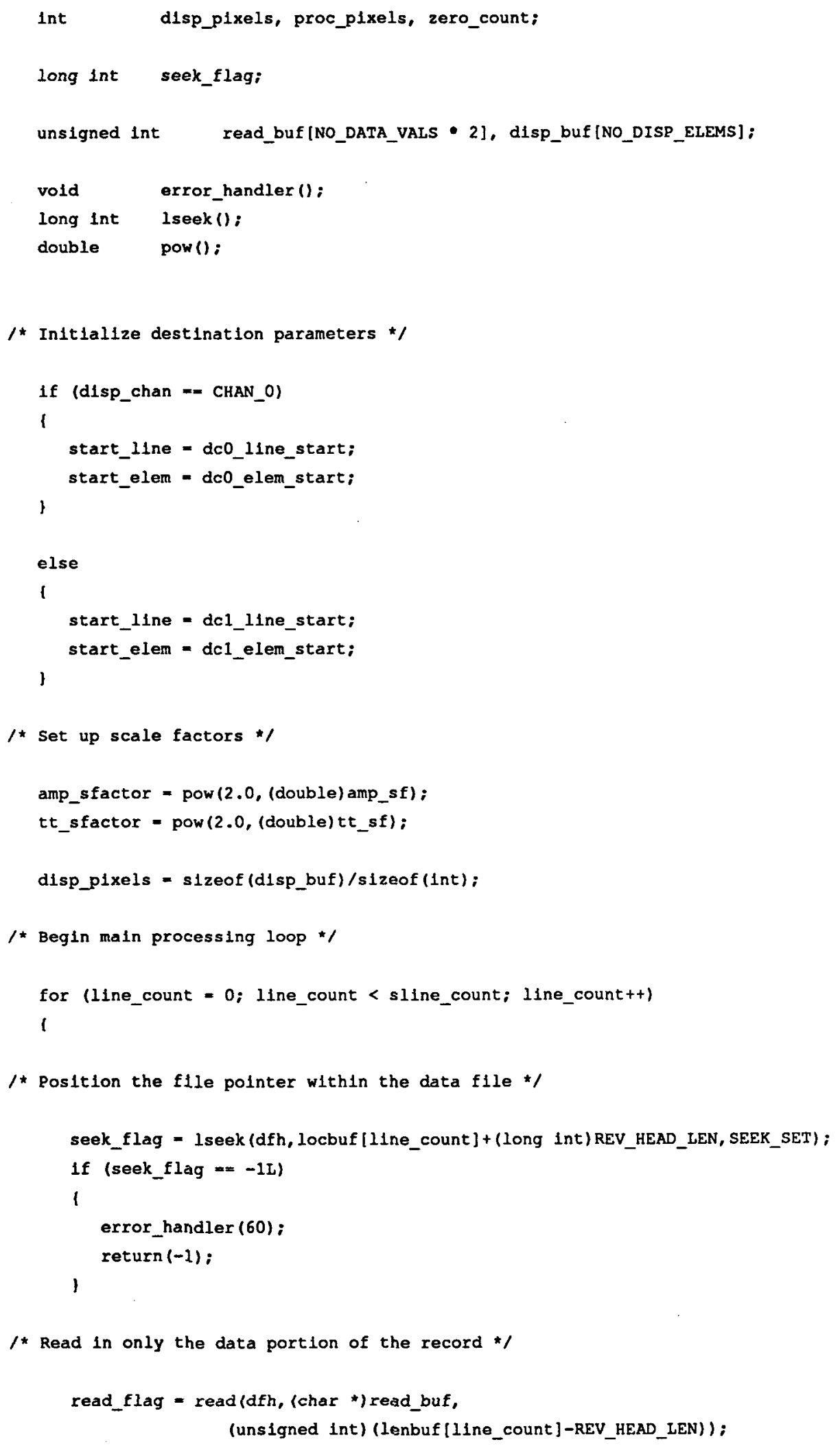




\section{Appendix J - BATD Source Code}

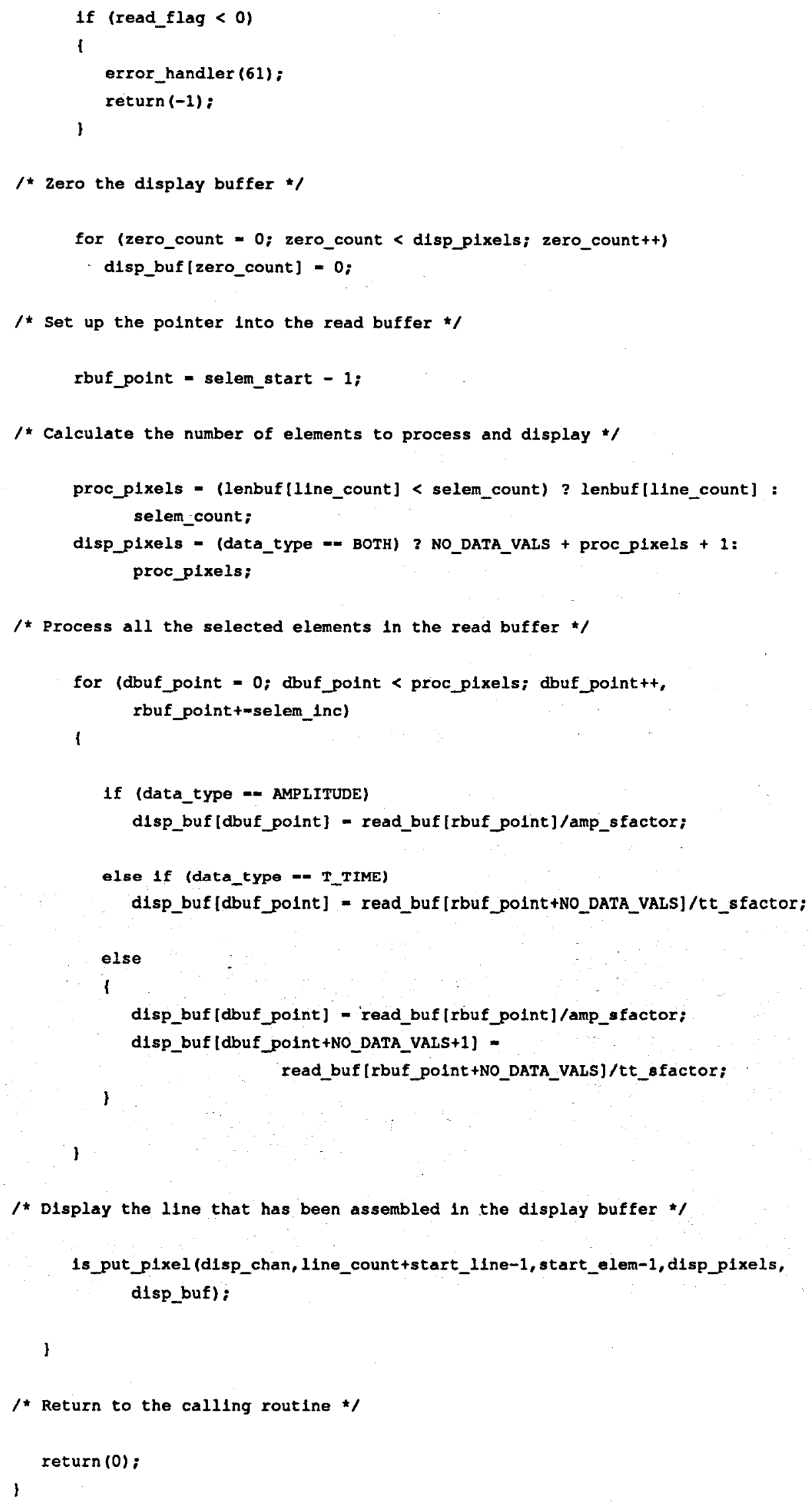




\section{Appendix K - BATT Source Code}

F1le: BATDDEFS.H

\begin{tabular}{|c|c|c|c|}
\hline define & VER & 1.00 & /* software version number * \\
\hline tdefine & VER_DATE & $n 11 / 13 / 87 n$ & \\
\hline tdefine & DISP_LINES & 480 & $/ * \max$ no of displayable lines */ \\
\hline fdefine & TRACES_DISP & 128 & $f^{*} \max$ no of traces per display $* /$ \\
\hline tdefine & MAX_DISPS & $\begin{array}{l}\prime \\
\prime\end{array}$ & $\begin{array}{l}\text { /* max no of successive displays */ } \\
/ * \text { used only to calculate RECS_SEL */ }\end{array}$ \\
\hline fdefine & RECS_SEL & TRACES_DIS & $\begin{array}{l}\text { SP MAX_DISPS } / * \max \text { no of traces } * / \\
/ * \text { or recs to process } * /\end{array}$ \\
\hline fdefine & MIN_INC & .5 & $f^{*}$ min Increment that w1ll be allowed */ \\
\hline Idefine & POINTS_PER_TRACE & 128 & /* data points per trace */ \\
\hline tefine & NO_RECS_PROC & 512 & $\begin{array}{l}/ * \text { no of recs to process in determining */ } \\
/^{*} \text { recs to use (select_recs) } * /\end{array}$ \\
\hline fdefine & CHECK_BAND & $\begin{array}{l}1 \\
\prime \\
\prime\end{array}$ & $\begin{array}{l}\text { /* used in select_recs to determine */ } \\
I^{*} \text { if local min is close enough to */ } \\
f^{*} \text { target depth to use } /\end{array}$ \\
\hline define & FNAME_LEN & 40 & $f *$ length of file name buffers */ \\
\hline fdefine & REV_HEAD & 48 & I* no bytes in rev header $* /$ \\
\hline tefine & CON_VAL & 508.0 & $\begin{array}{l}1 * \text { conversion from callper in } \mathrm{mm} \times 10 * / \\
/ * \text { to radius in inches */ }\end{array}$ \\
\hline Hdefine & PIXELS_PER_POINT & ' & $\begin{array}{l}\text { /* no of columns over which to display */ } \\
1 * \text { each callper value */ }\end{array}$ \\
\hline define & START_COLUMN & 140 & $\begin{array}{l}\text { /* display column to place first data */ } \\
/ * \text { value */ }\end{array}$ \\
\hline tefine & TRACE_INTENSITY & 255 & 1* Intensity to draw traces */ \\
\hline define & TICK_LINE_INTENSIT & TY 100 & /* Intensity to draw tick lines $\star /$ \\
\hline Idefine & TEXT_INTENSITY & 150 & $f^{*}$ Intensity to print text */ \\
\hline define & DISP_FRAME & 0 & $I^{*}$ frame buffer to use * \\
\hline \multicolumn{4}{|c|}{ I* Annotation positioning parameters } \\
\hline define & DEPTH_COL & 8 & $f^{*}$ display column to start depth info */ \\
\hline define & REF_COL & 100 & $1 *$ display column to start tick line info */ \\
\hline tefine & MIN_COL & 410 & /* display column to start min val info */ \\
\hline tdefine & MAX_COL & 472 & $f^{*}$ display column to start max val Info */ \\
\hline Adefine & HALF_CHAR & 1 & $\begin{array}{l}1 * \text { offset from center line to position */ } \\
/ * \text { text } * /\end{array}$ \\
\hline
\end{tabular}

/* Set up and name the structure templates */

typedef struct

$\begin{array}{ll}\text { long int } & \text { rec_offset; } \\ \text { float } & \text { depth; } \\ \text { lnt } & \text { rec_length; }\end{array}$




\section{Appendix K - BATT Source Code}

$\begin{array}{llll}\text { typedef struct } \quad\{ & & \\ & \text { float } & \text { depth; } \\ & \text { long 1nt } & \text { rec_no; } \\ & \text { float } & \text { min; } \\ & \text { float } & \text { max; } \\ & \text { f } & \text { SEL_REC; } & \end{array}$

FIle: BATTGLOB. H

char data_fname [FNAME_LEN], dp_fname [FNAME_LEN] ;

int no_traces, no_disps, traces_per_disp;

int dfh, dpfh, rsel_flag, run_flag;

Int t1ck_IInes [TRACES_DISP+2] [2], map_data [POINTS_PER_TRACE] [2];

float flrst_file_depth, last_flle_depth, dlines_per_inch;

double Inlt_depth, fin_depth, Inc_depth, base_tlc, t1c_res;

REC_INFO Info_buf, *Info_buf_ptr;

SEI_REC selected_recs [RECS_SEL];

F1le: BATTWND.H

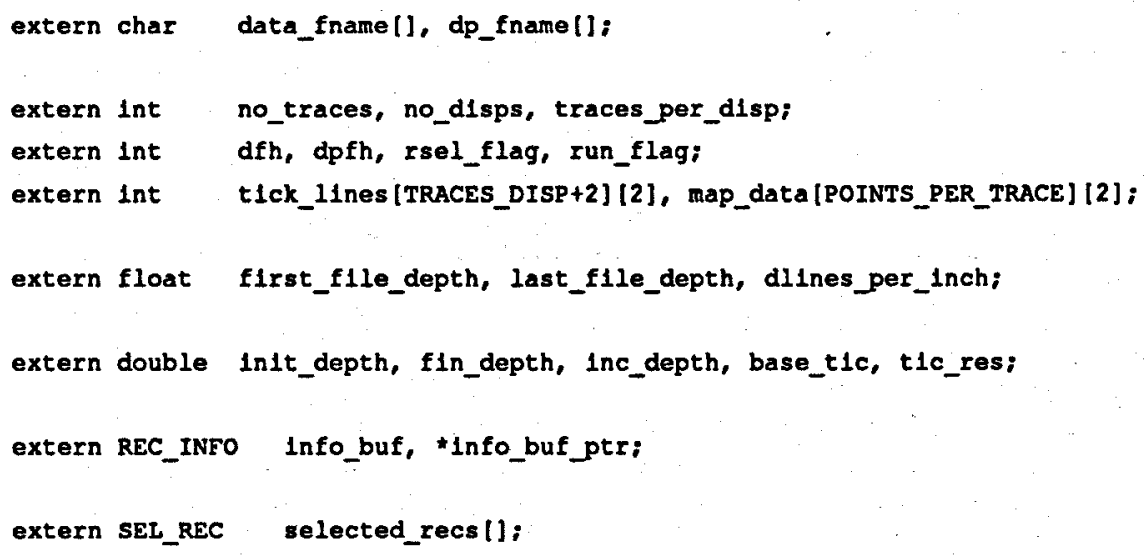




\section{Appendix K - BATT Source Code}

FIle: BATTXTRN.H

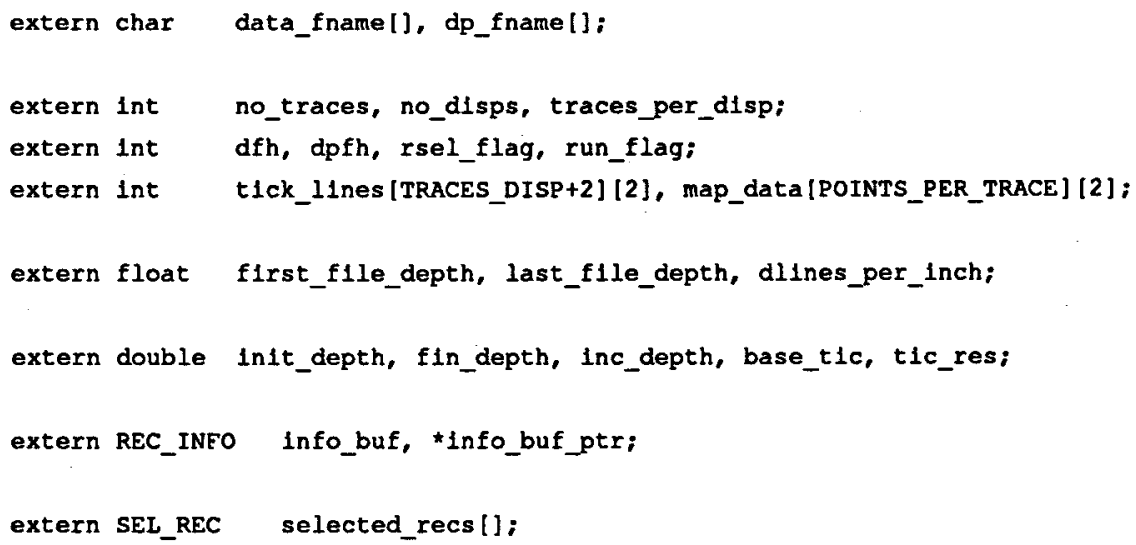

F1le: BATTXWND.H

Hnclude $\langle w f d . h\rangle$

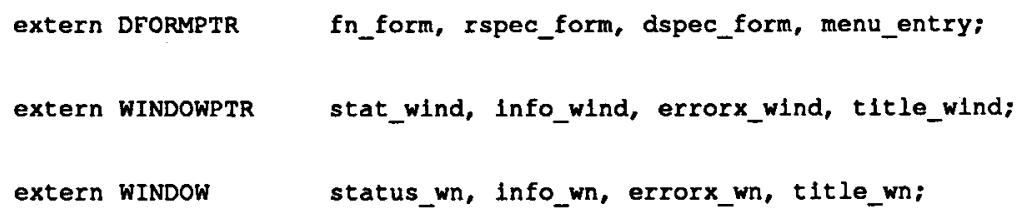

Flle: BATD.C

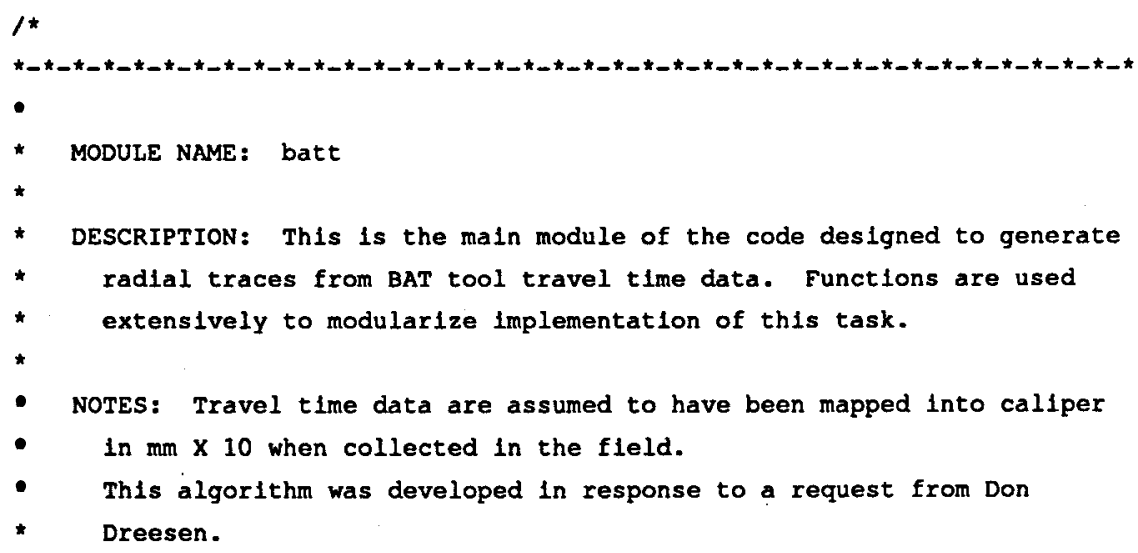




\section{Appendix K - BATT Source Code}

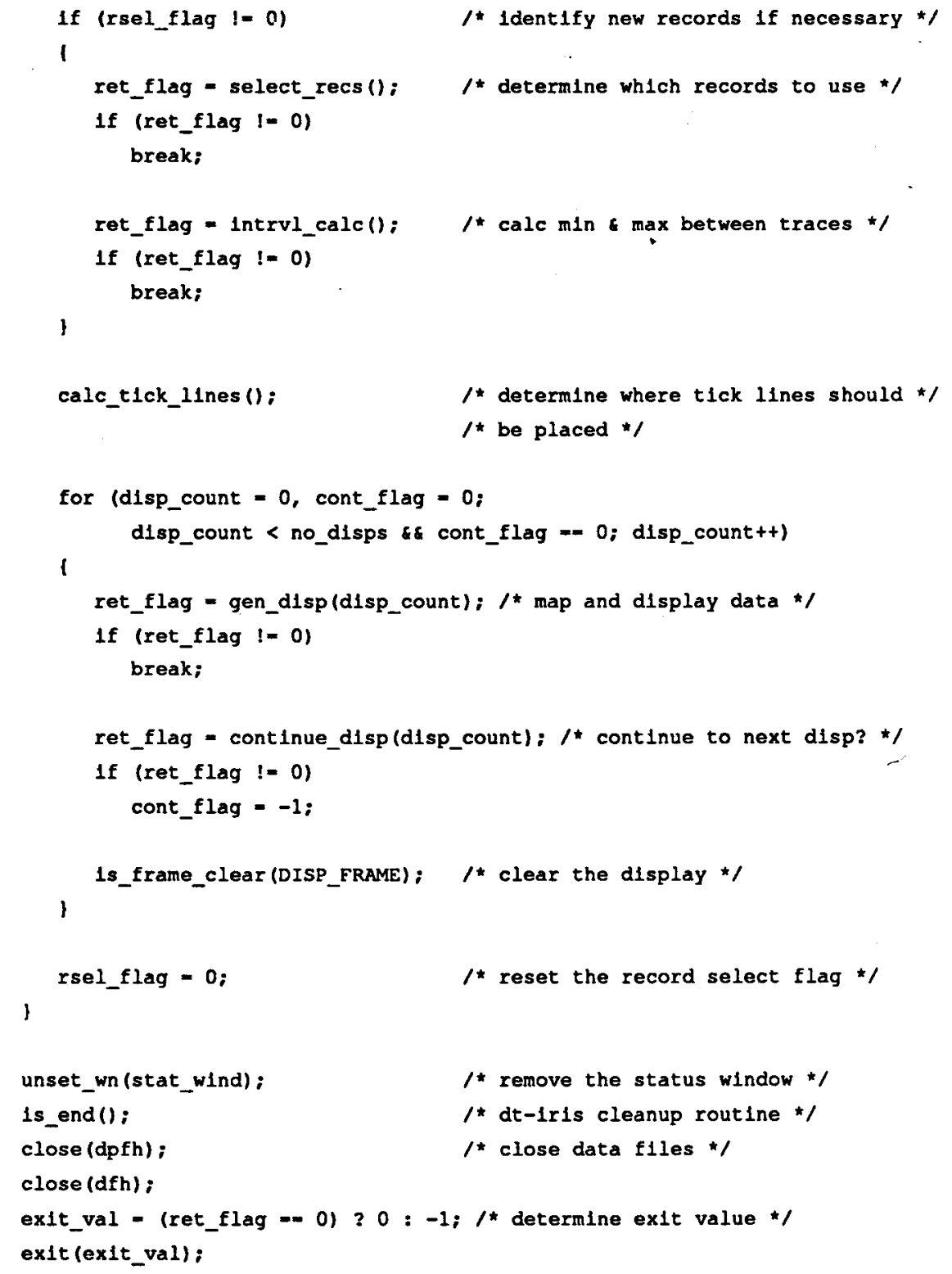




\section{Appendix K - BATT Source Code}

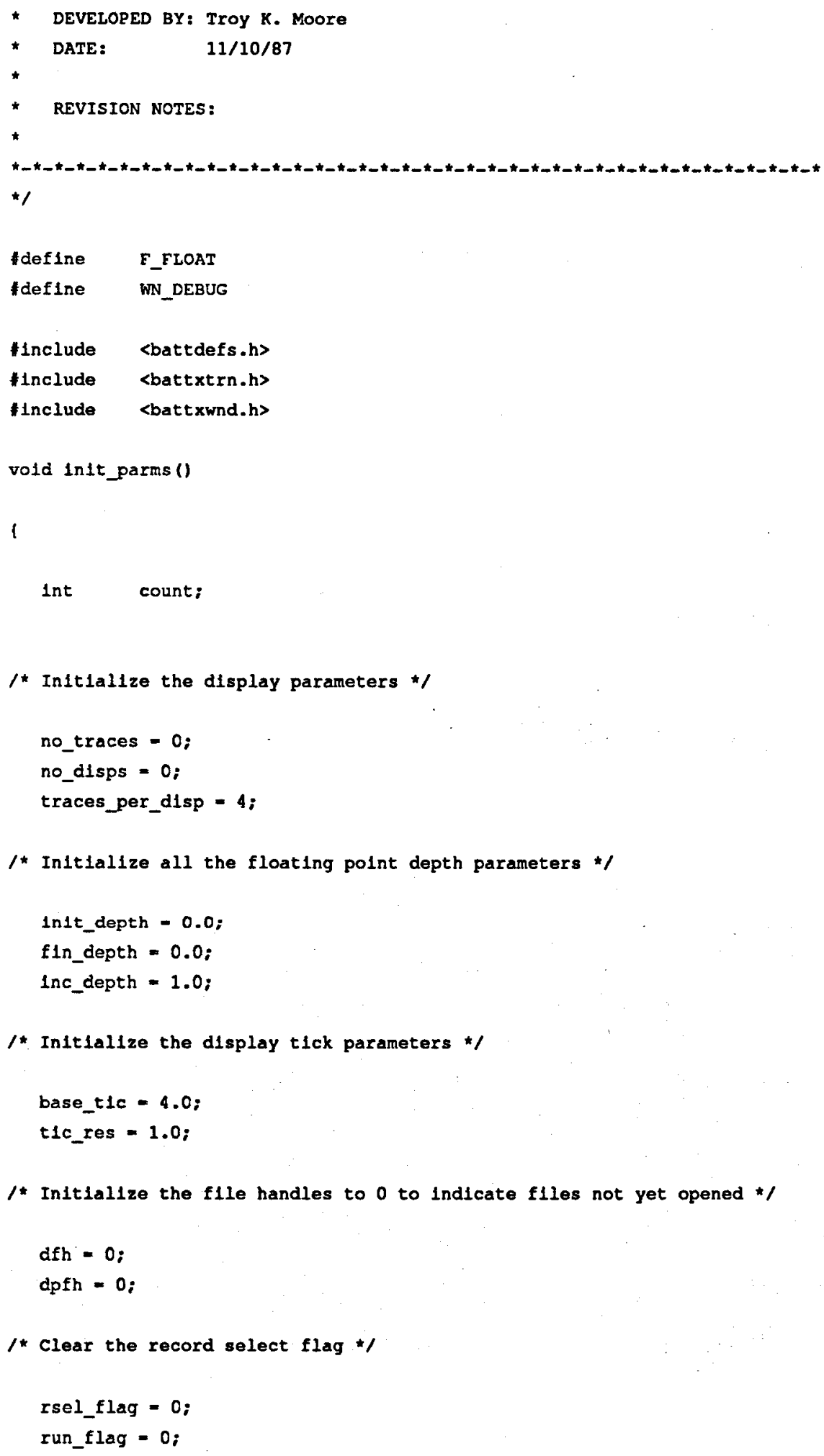




\section{Appendix K - BATT Source Code}

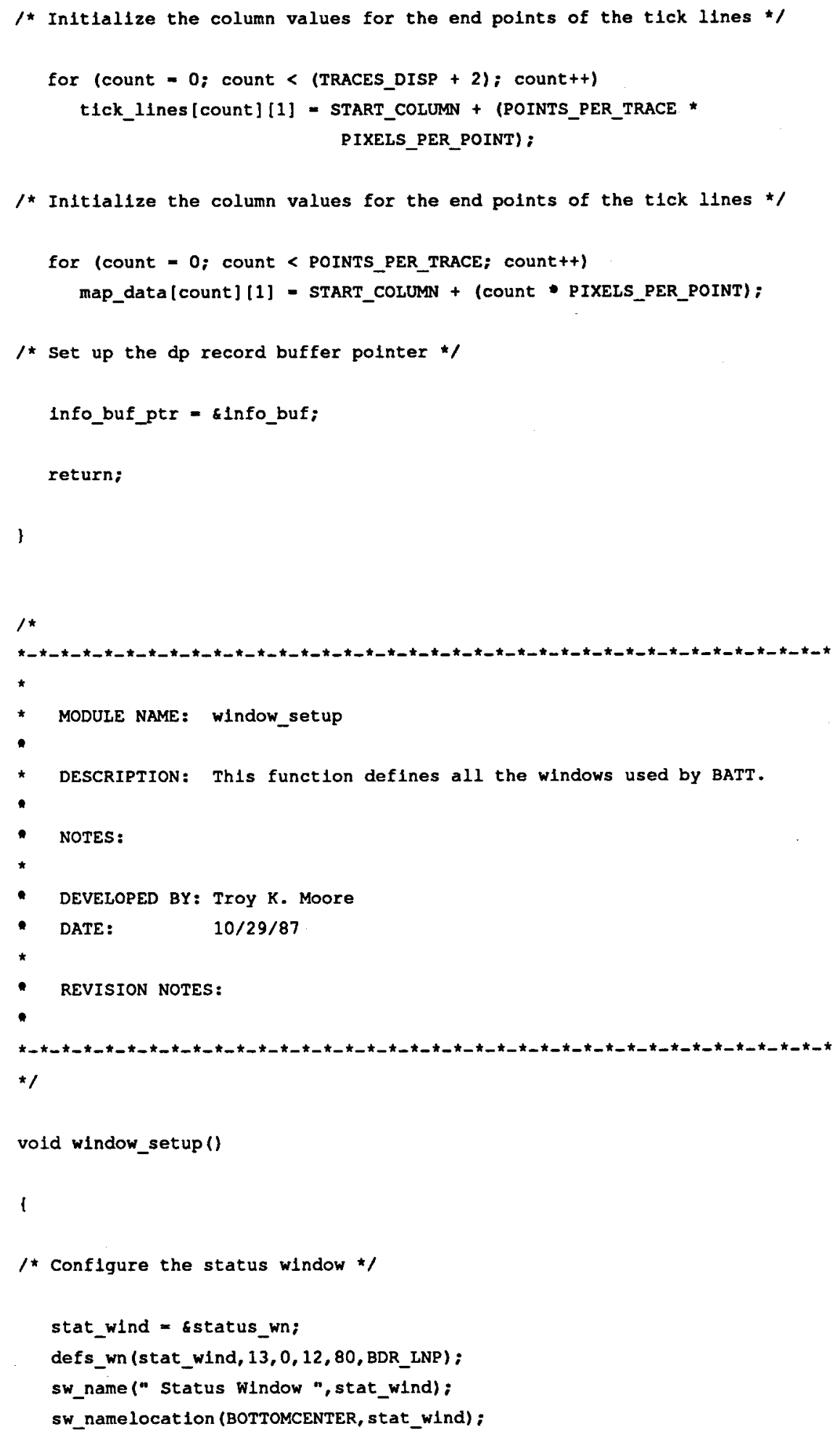




\section{Appendix K - BATT Source Code}

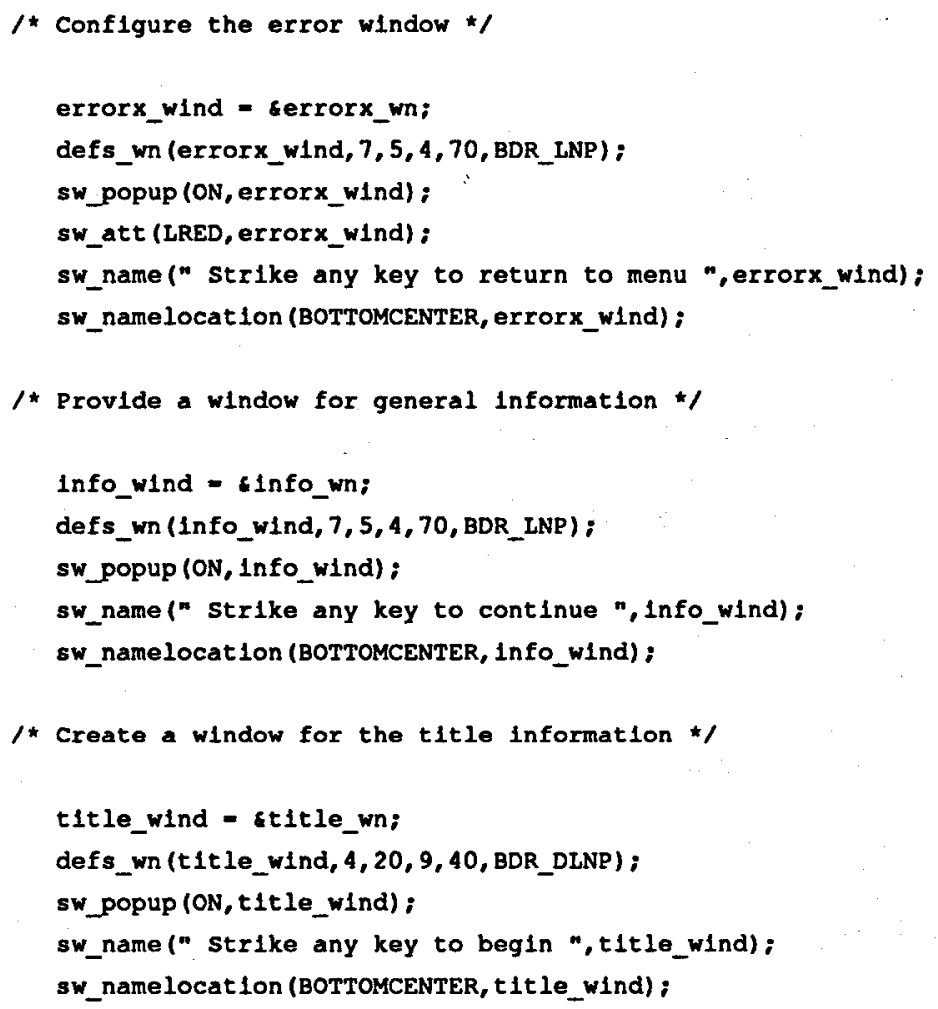




\section{Appendix K - BATT Source Code}

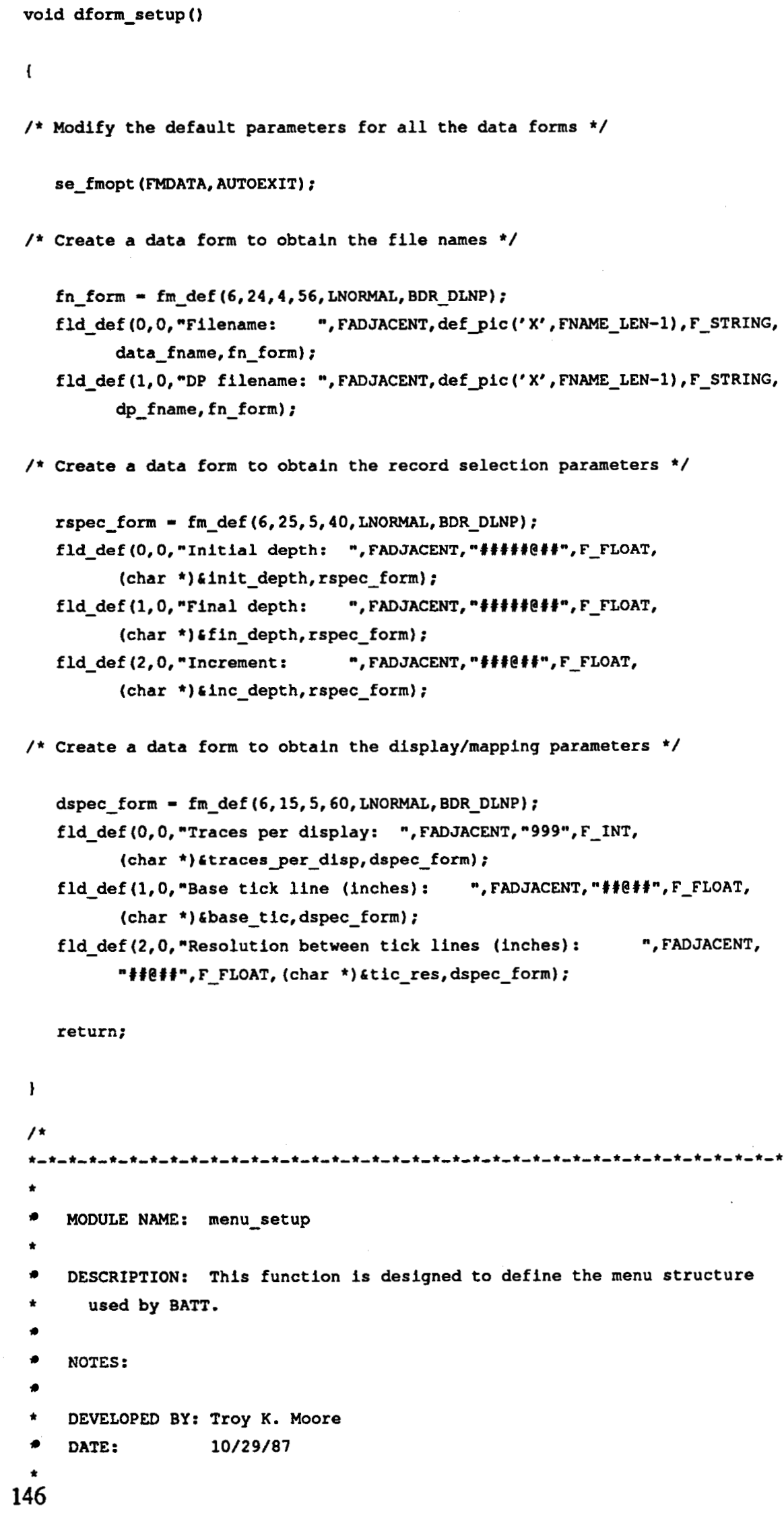




\section{Appendix K - BATT Source Code}

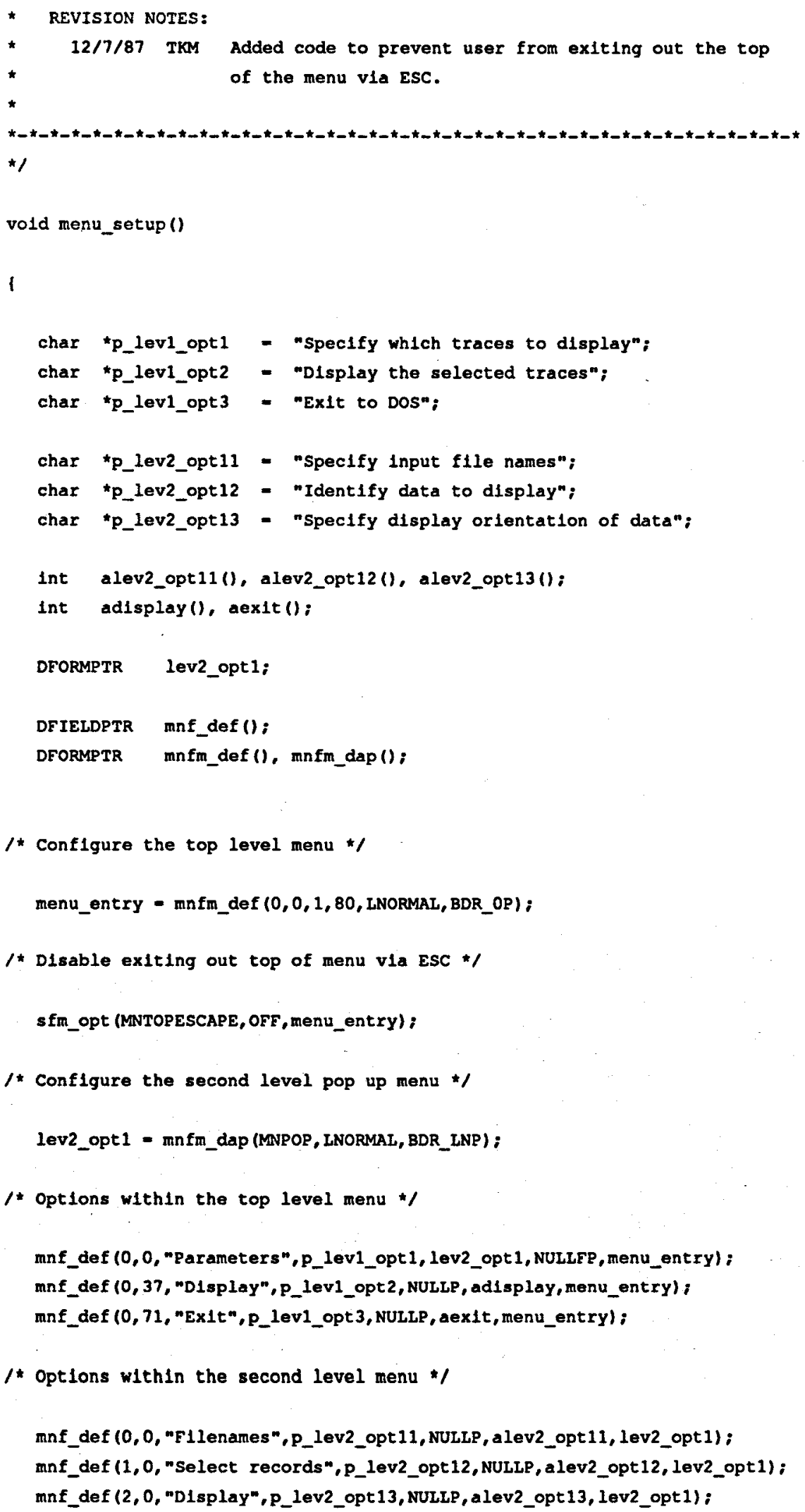




\section{Appendix K - BATT Source Code}

return;

\}

F1le: BATTWIND.C

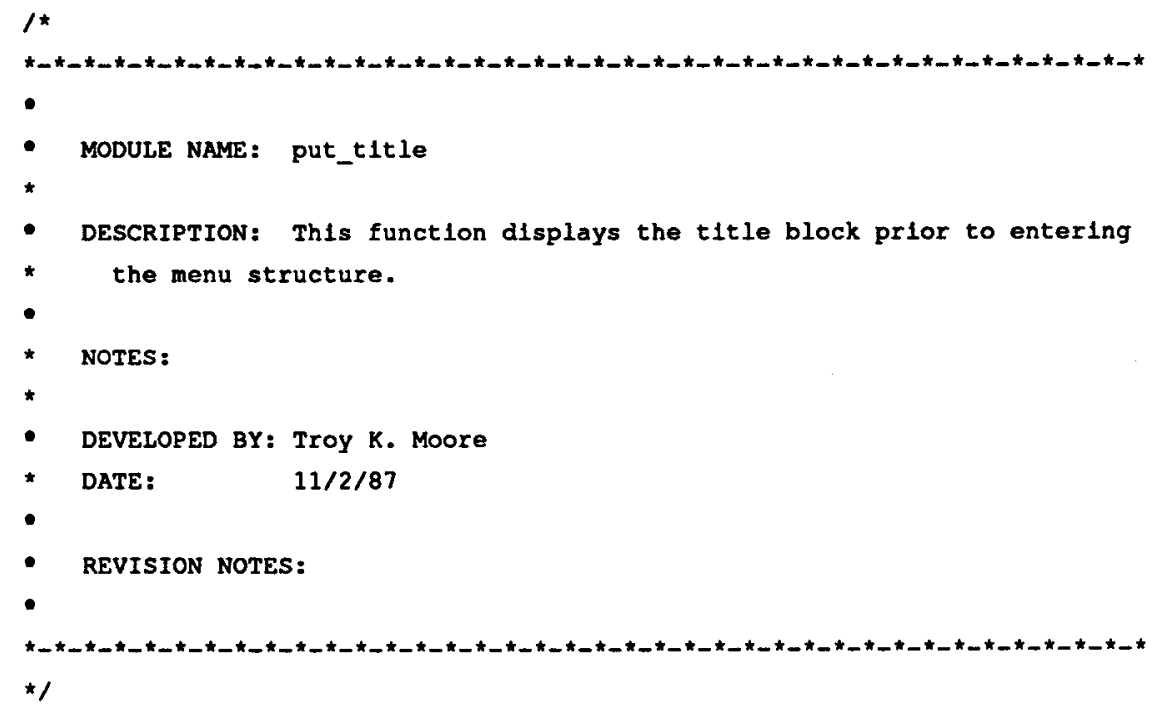




\section{Appendix K - BATT Source Code}

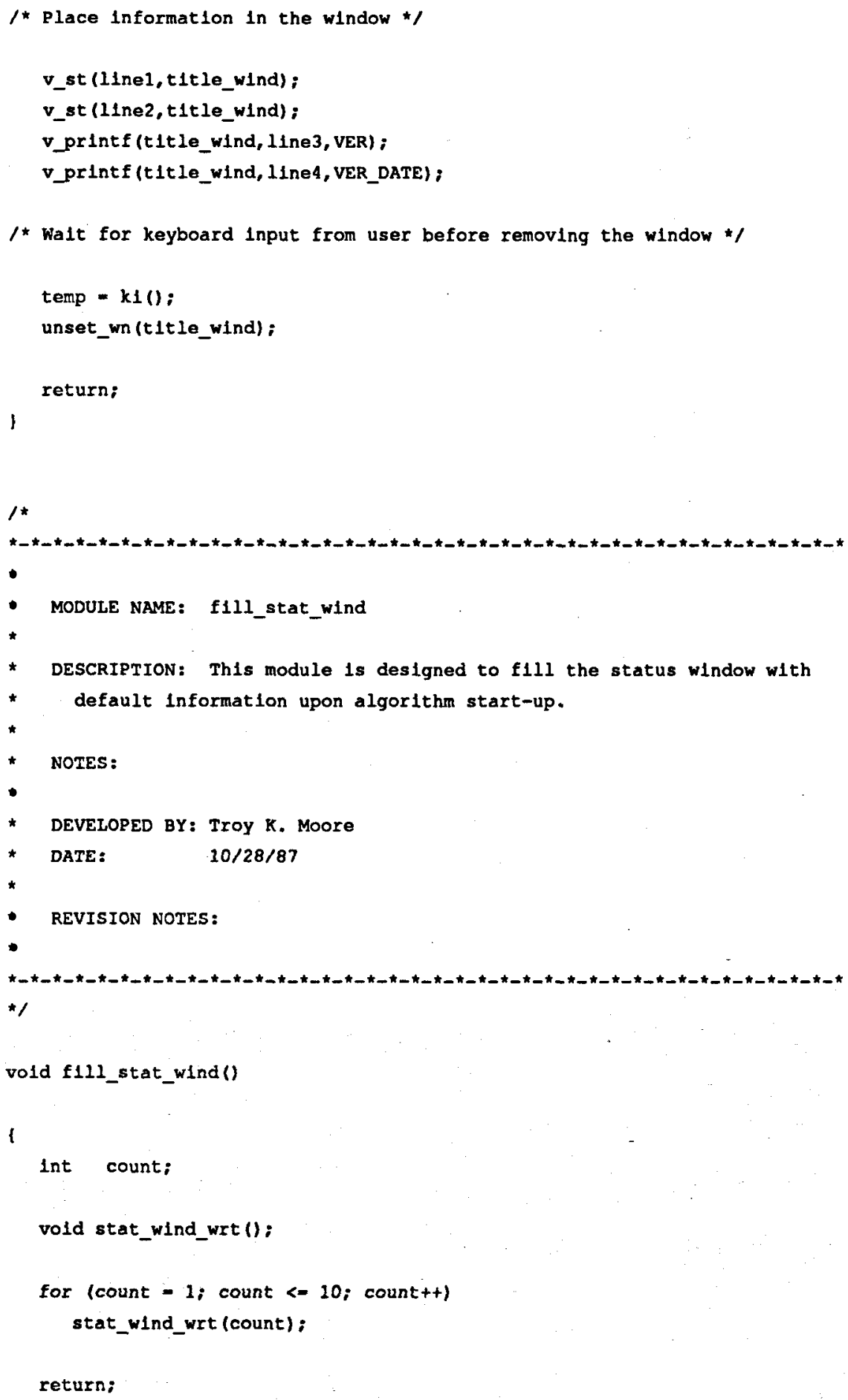




\section{Appendix K - BATT Source Code}

$1 *$

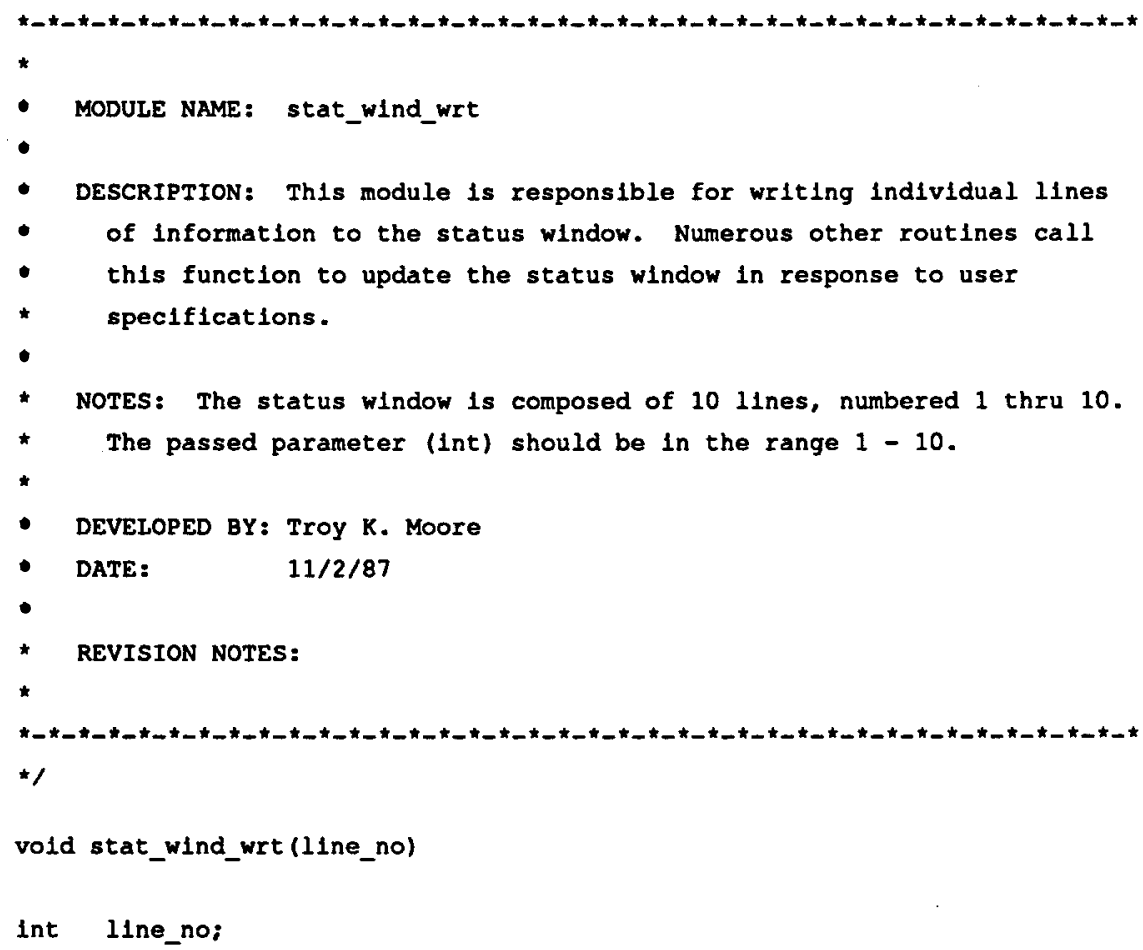

/* Update the line in the status window based on the passed value */

switch (11ne_no)

I

case 1:

$1 *$ Line $1 * /$

mv_cs $(0,0$, stat_wind);

v_st ("Source", stat_wind);

break;

case 2:

$1 *$ Line $2 * /$

mv_cs $(1,0$, stat_wind $)$ :

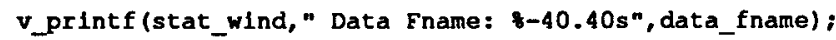

break;

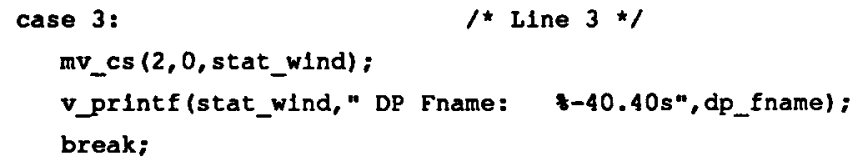
last_flle_depth,first_flle_depth): 


\section{Appendix K - BATT Source Code}

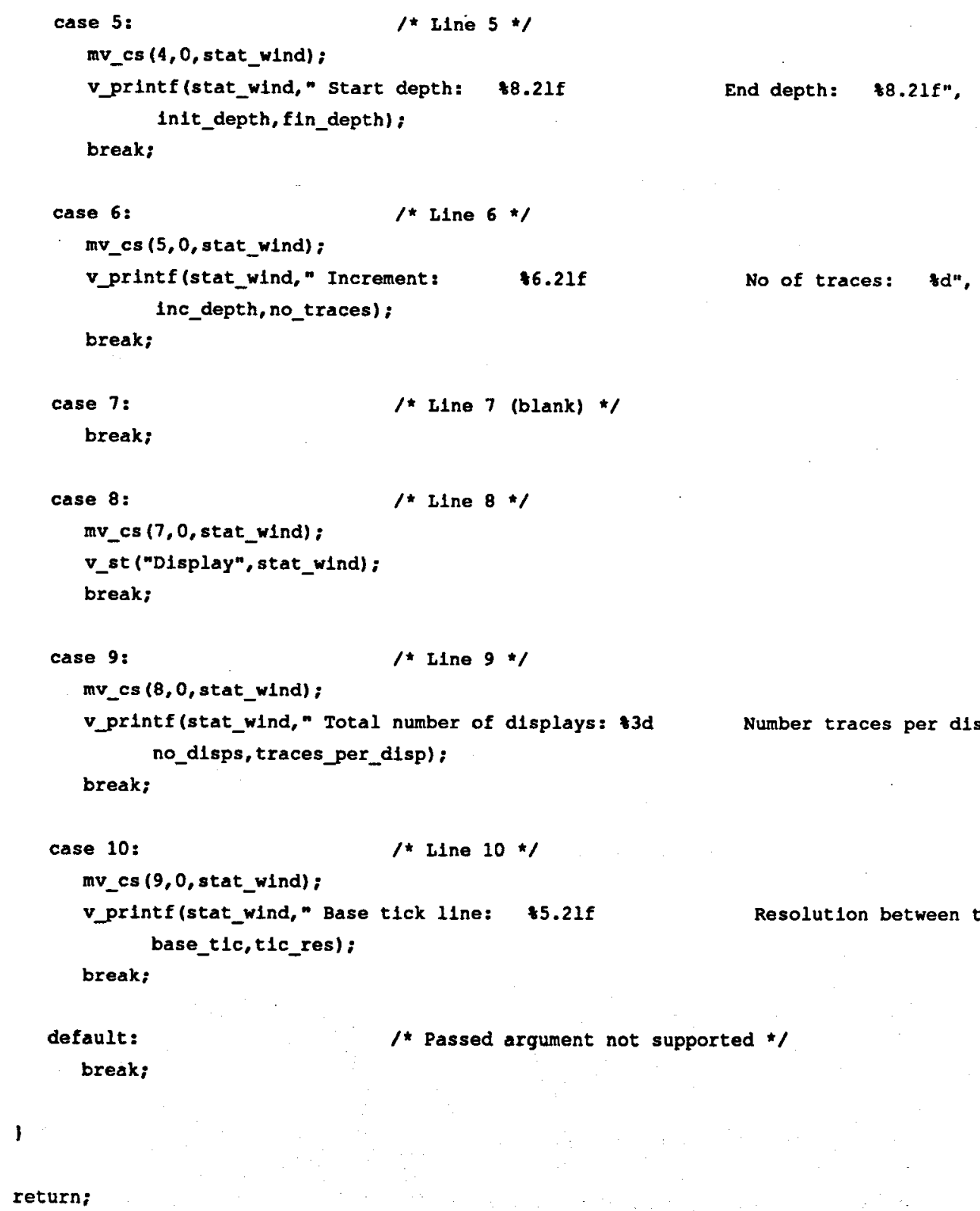




\section{Appendix K - BATT Source Code}

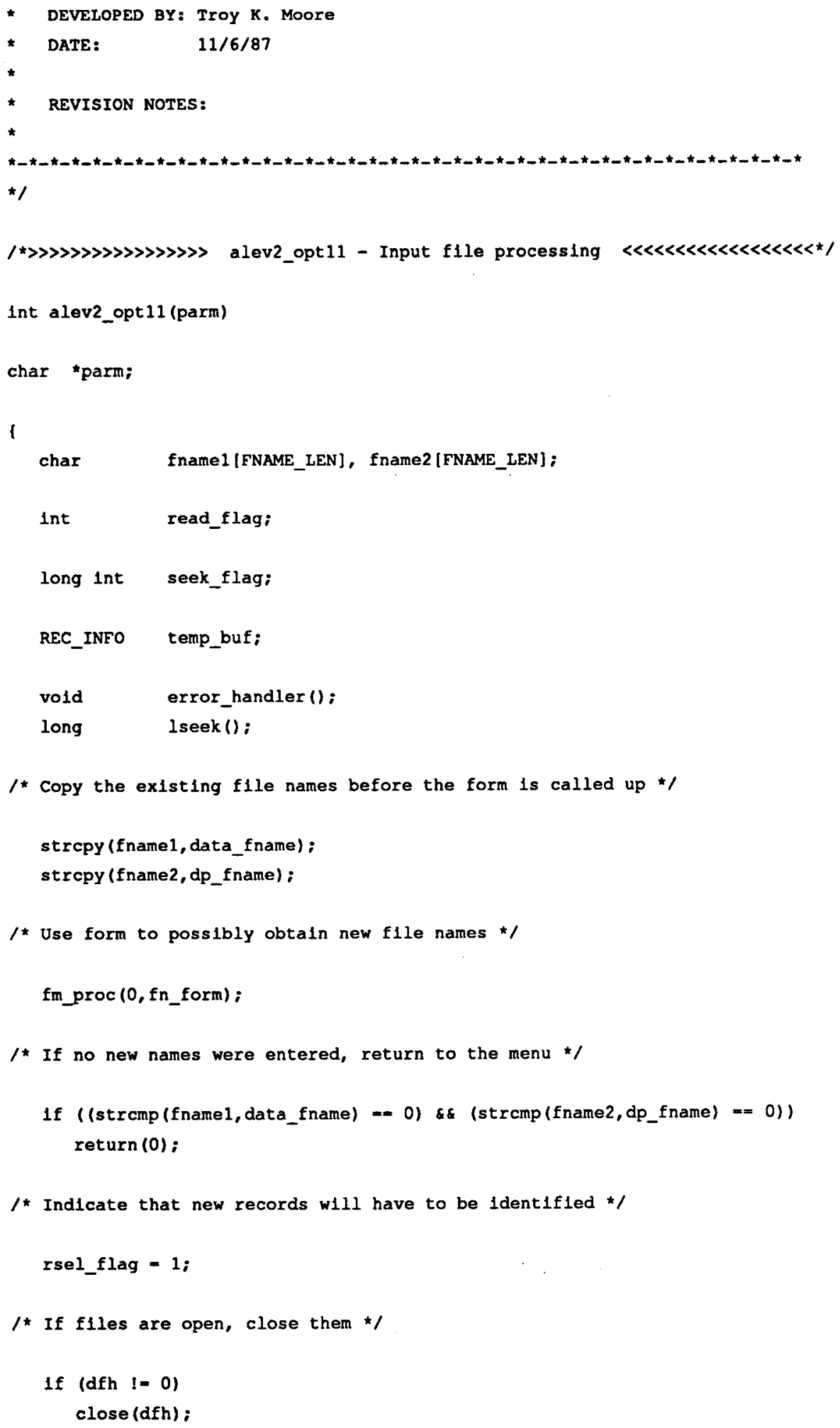




\section{Appendix K - BATT Source Code}

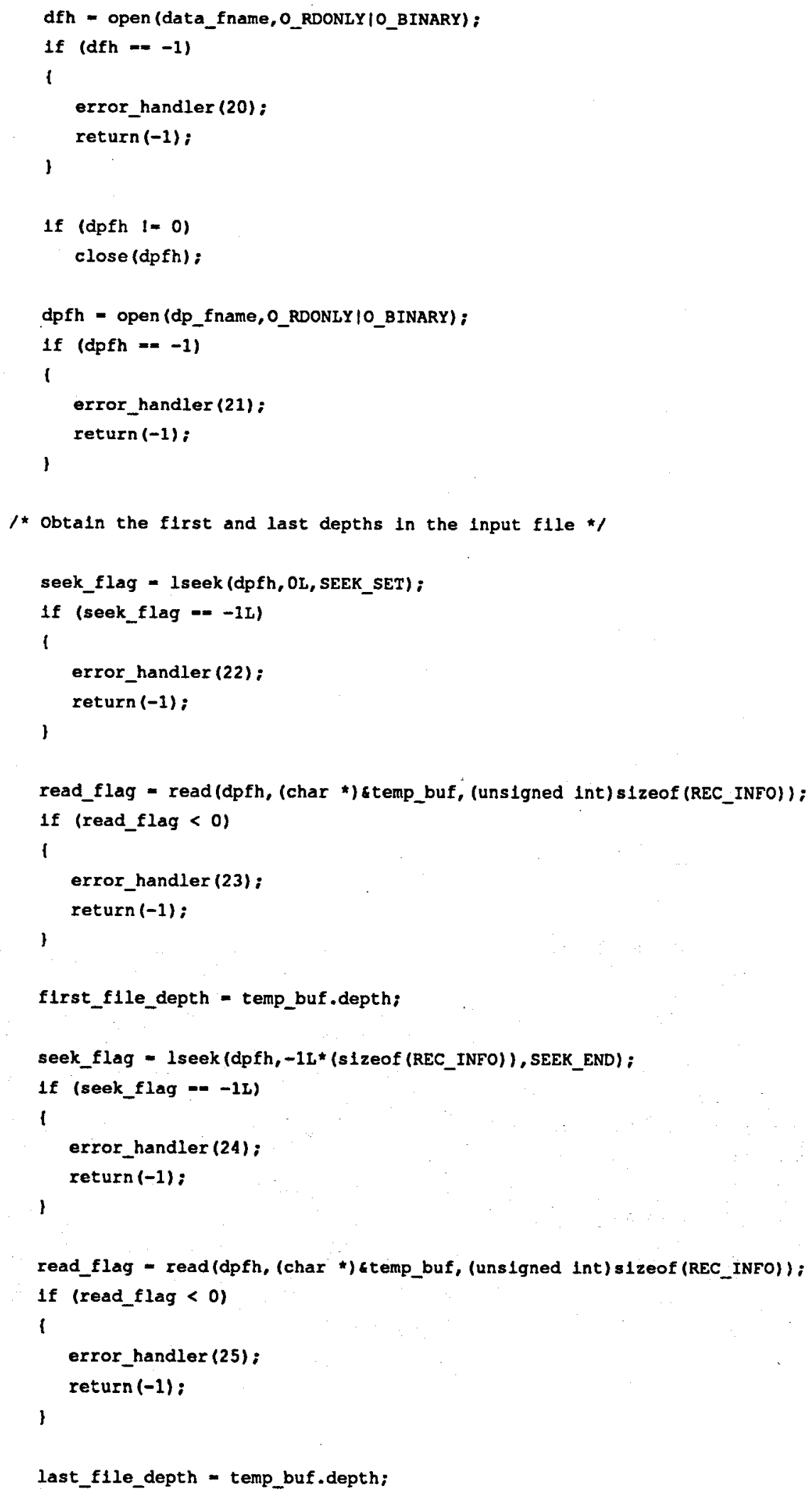




\section{Appendix K - BATT Source Code}

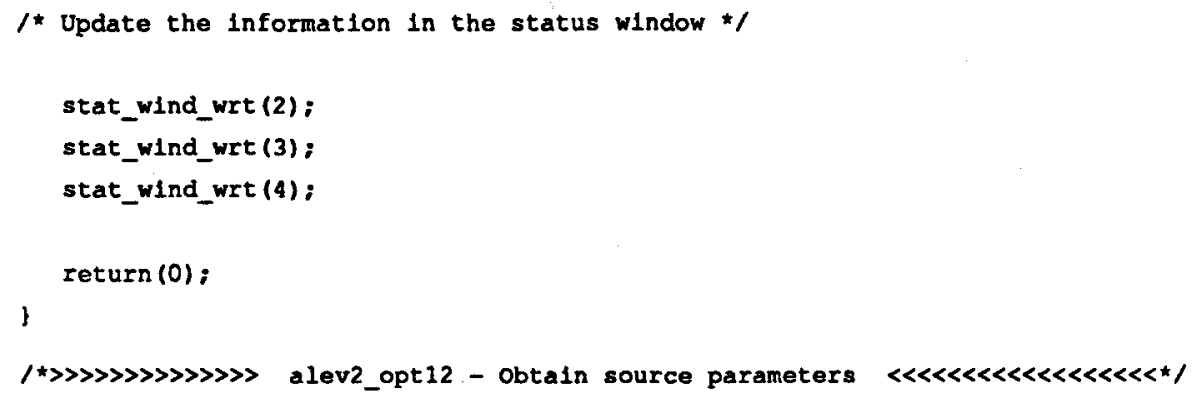




\section{Appendix K - BATT Source Code}

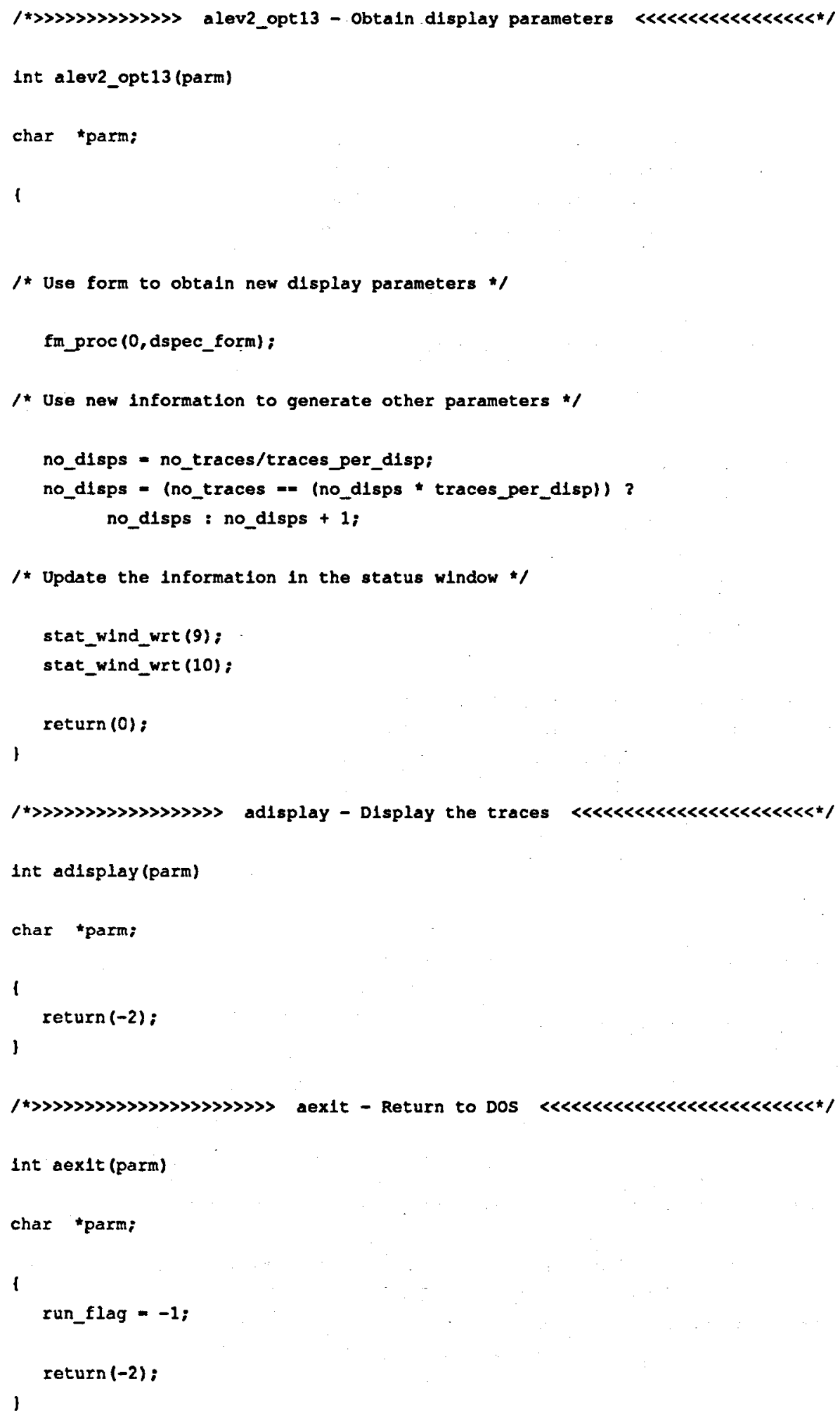




\section{Appendix K - BATT Source Code}

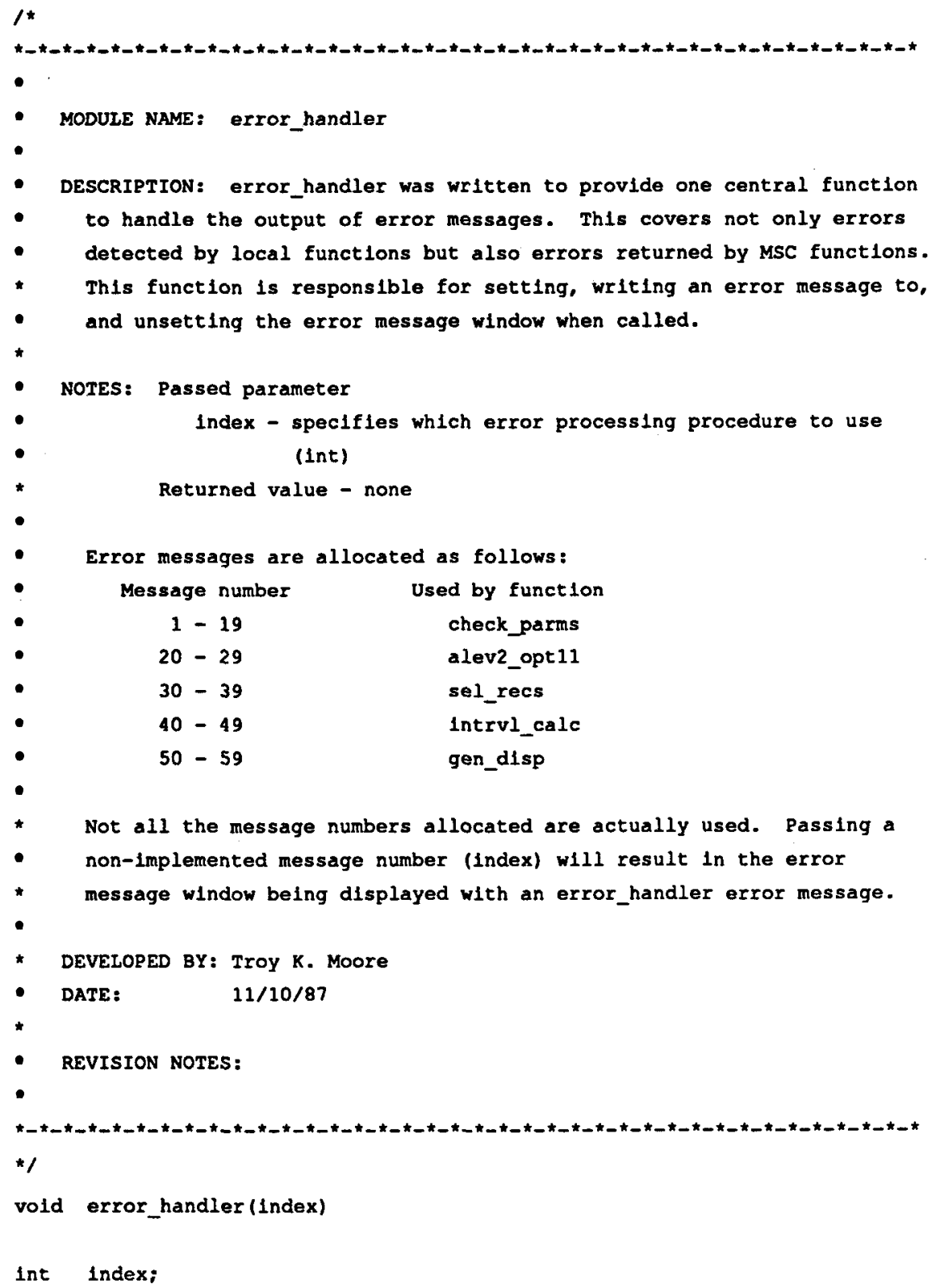

Not all the message numbers allocated are actually used. Passing a non-implemented message number (Index) will result in the error message window being displayed with an error_handler error message.

-

* DEVELOPED BY: TrOY K. Moore

- DATE: $11 / 10 / 87$

$\star$

- REVISION NOTES:

-

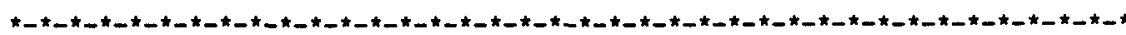

*/

vold error_handler(Index)

int index;

1

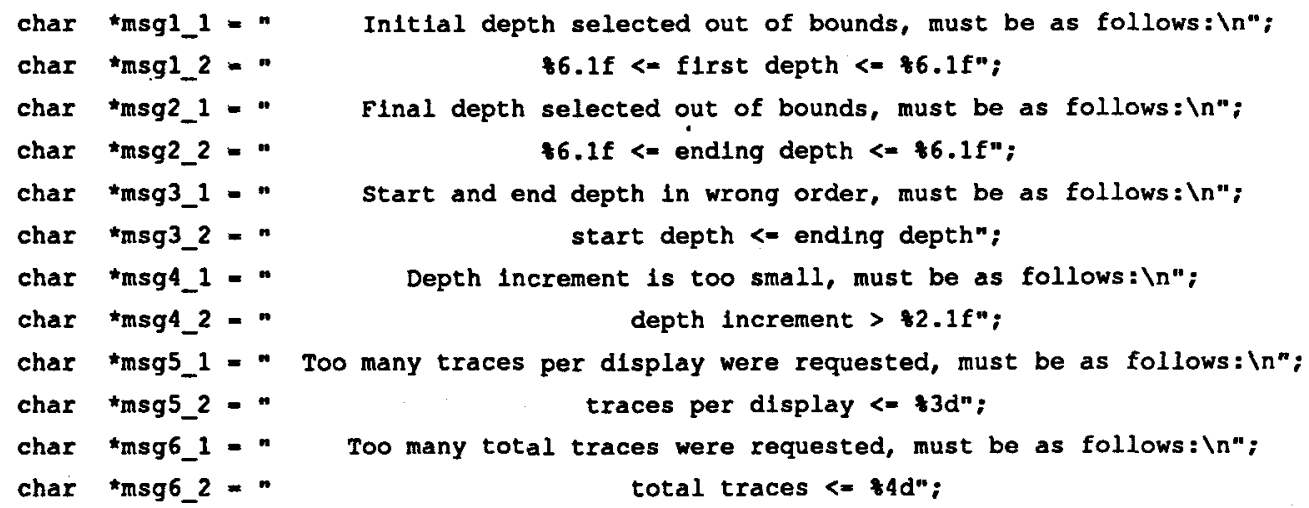

Initial depth selected out of bounds, must be as follows: In";$$
\$ 6.1 \mathrm{f}=\text { flrst depth }<=\$ 6.1 \mathrm{f} " ;
$$ 


\section{Appendix K - BATT Source Code}

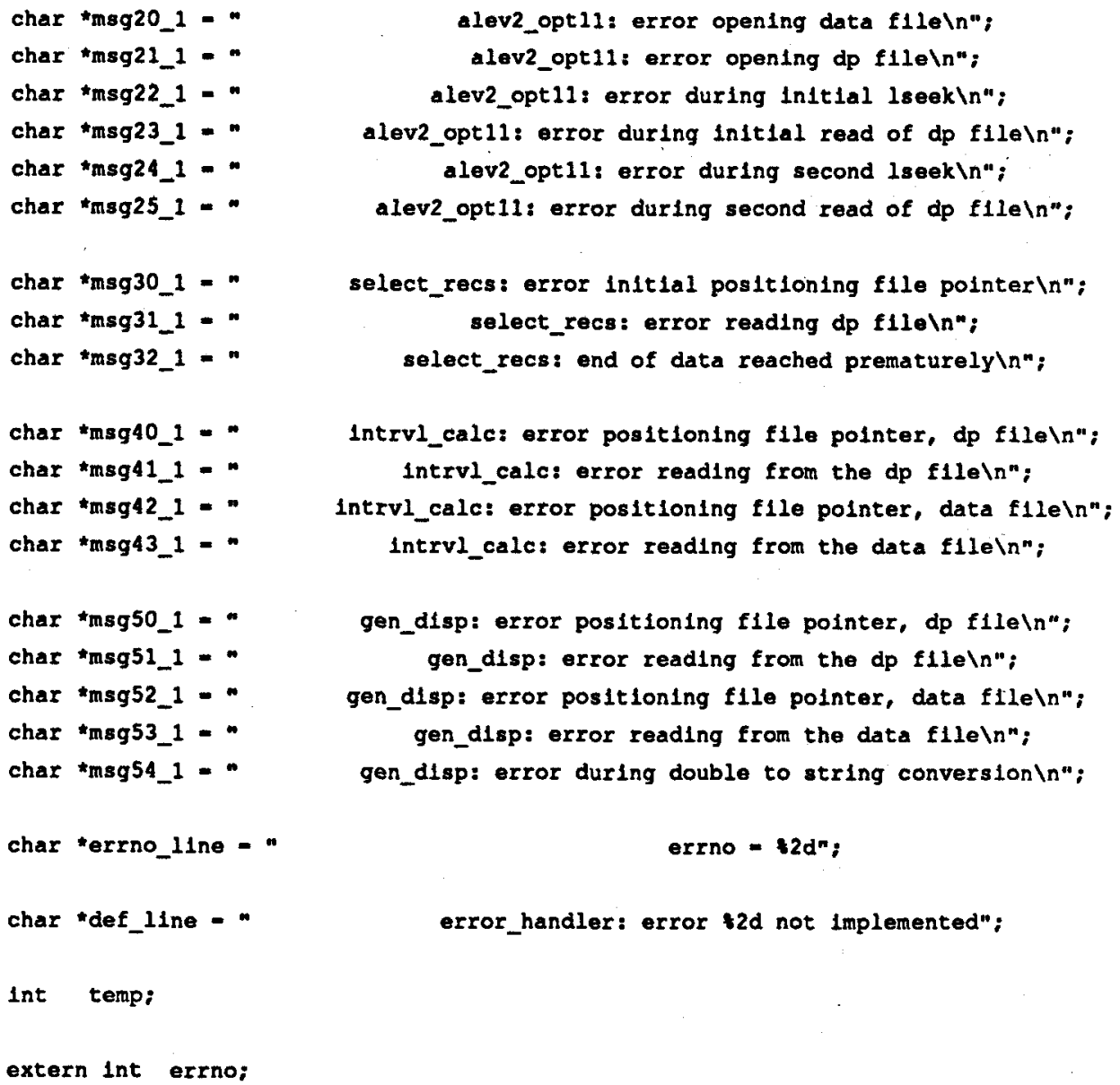




\section{Appendix K - BATT Source Code}

case 3:

v_st (msg3_1, errorx_wind):

v_st (msg3_2, errorx_wind);

break:

case 4:

v_st (msg4_1, errorx_wlind):

v_printf (errorx_wind, msg4_2,MIN_INC);

break:

case 5:

v_st (msg5_1, errorx_wind) :

v_printf (errorx_wind,msg5_2,TRACES_DISP);

break:

case 6:

v_st (msg6_1,errorx_wind) ;

v_printf (errorx_wind, msg6_2,RECS_SEI) ;

break:

case $20:$

v_st (msg20_1, errorx_wind);

v_printf (errorx_wind,errno_line,errno);

break;

case 21:

$v$ st (msg21_1, errorx_wind):

v_printf (errorx_wind, errno_line,errno);

break;

case 22:

v_st (msg22_1, errorx_w1nd) :

$v$ printf (errorx_wind, errno_line, errno):

break;

case 23:

v_st (msg23_1,errorx_wind) :

v_printf (errorx_wind, errno_line, errno) ;

break:

case 24:

v_st (msg24_1, errorx_w1nd):

v_printf (errorx_wind, errno_11ne,errno);

break:

case 25 :

v_st (msg25_1, errorx_wind) :

v_printf (errorx_wind, errno_11ne, errno);

break;

case 30:

v_st (msg30_1, errorx_w1nd);

$v$ printf (errorx_wind, errno_line,errno) ; break; 


\section{Appendix K - BATT Source Code}

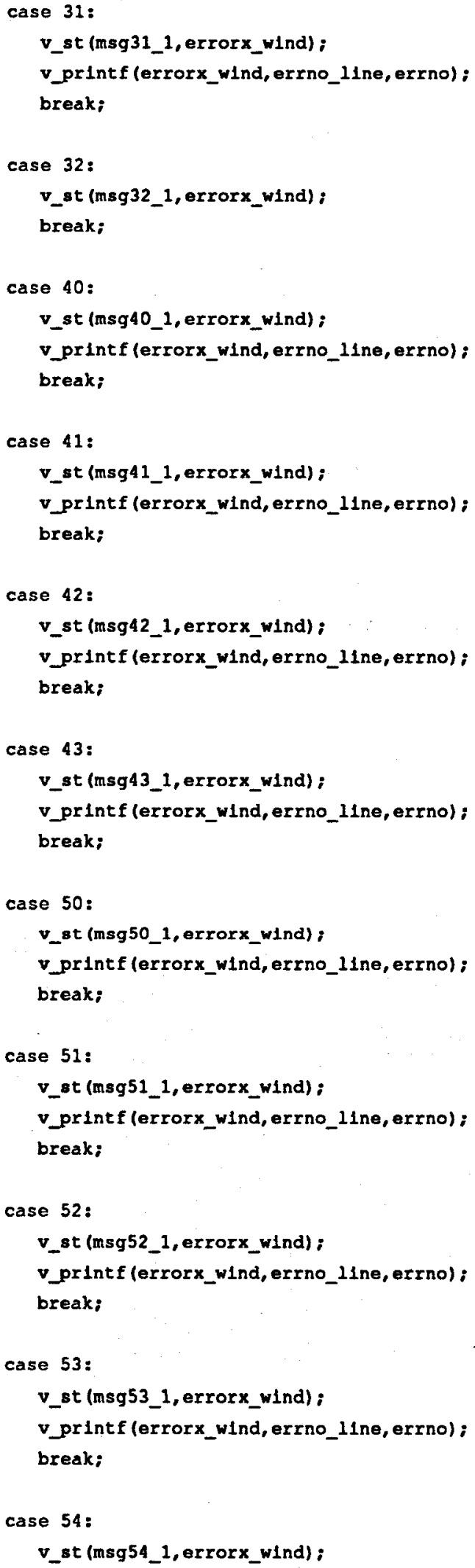




\section{Appendix K - BATT Source Code}

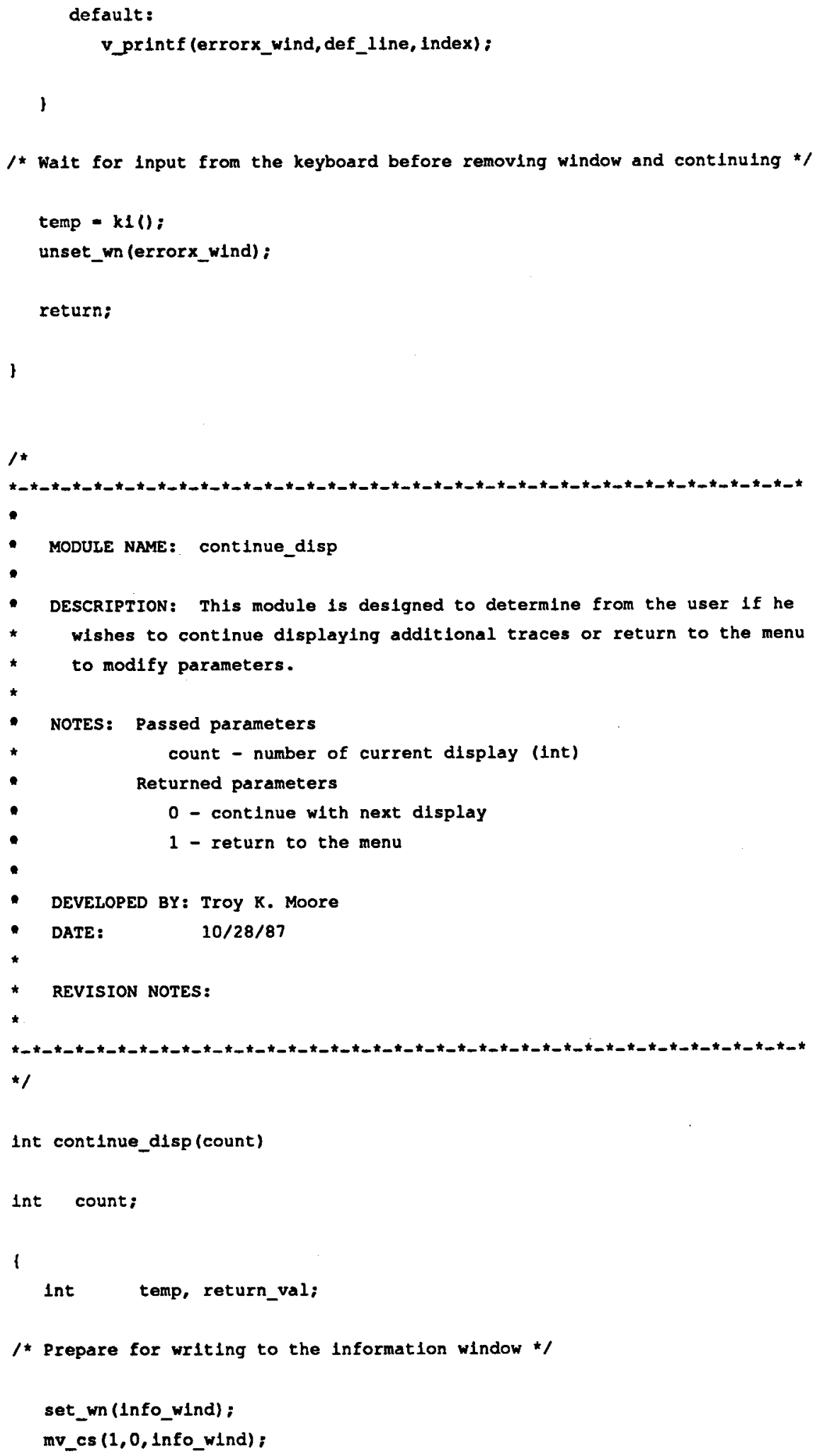




\section{Appendix K - BATT Source Code}

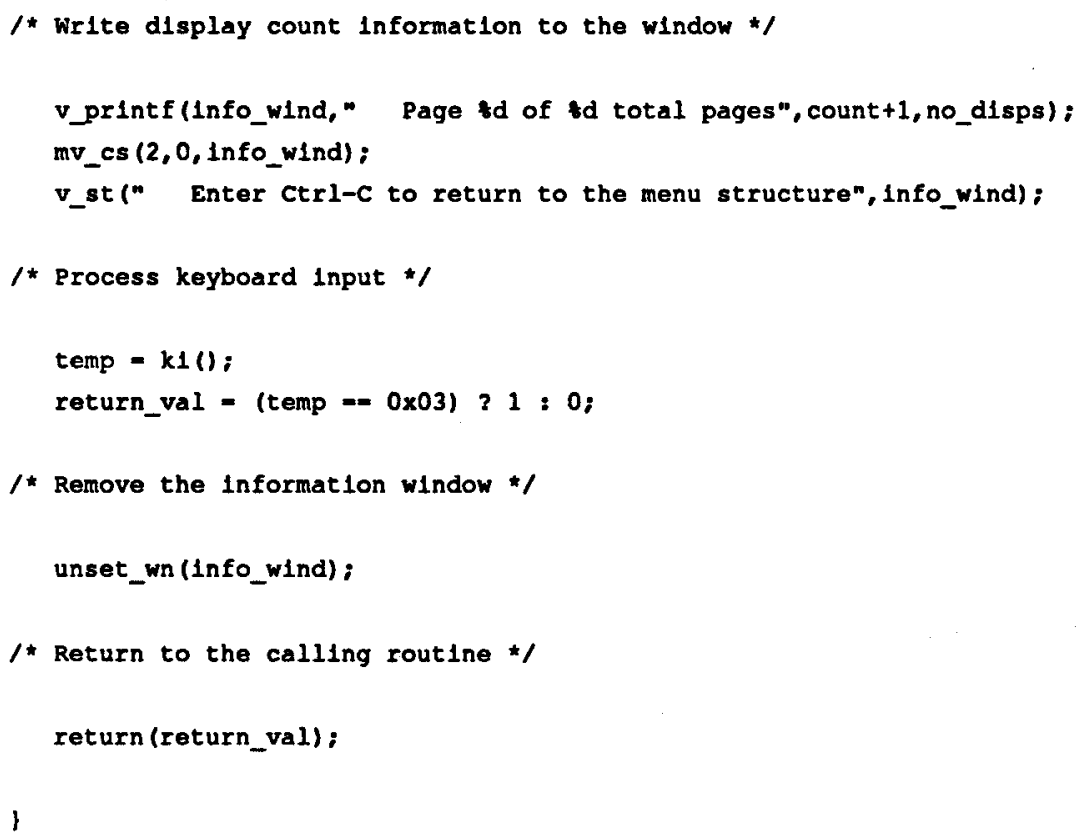




\section{Appendix K - BATT Source Code}

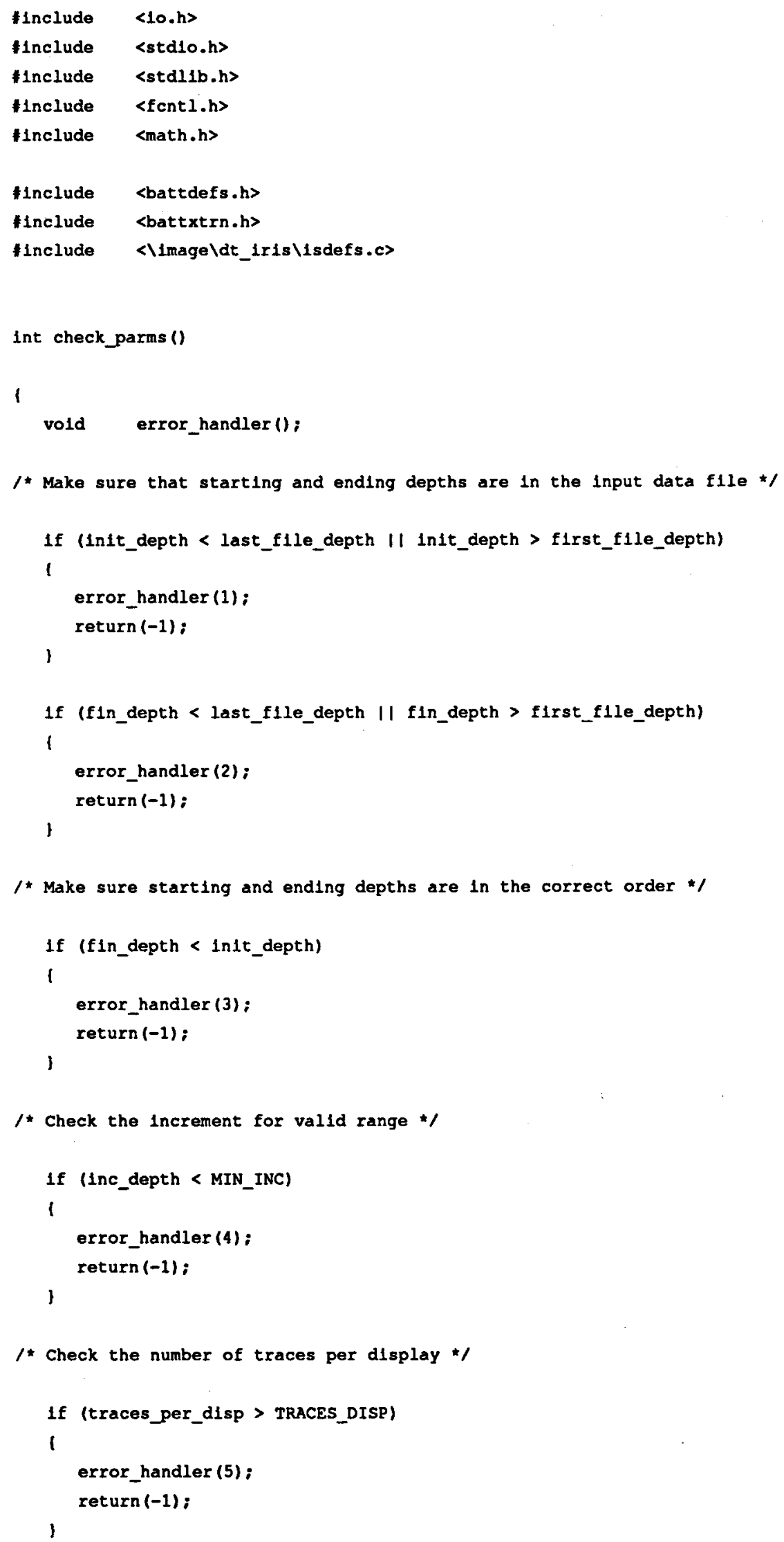




\section{Appendix K - BATT Source Code}

I* Check the total number of traces to process */

If (no_disps > RECS_SEL)

I

error_handler (6) :

return $(-1)$ :

l

/* Return without error if this polnt is reached */

return (0):

$1 *$

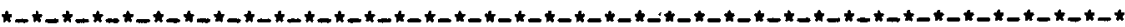

MODULE NAME: select_recs

DESCRIPTION: Th1s function 1s deslgned to select records from the data

- flle that most closely match the depth specifications given by the

* user. The record number of each selected record is stored in a

- structure containing information assoclated with each record used.

* Records are selected based on the absolute value of the difference

*

NOTES: select_recs $1 \mathrm{~s}$ a modifled version of the function depth_rec_sel used by BATD.

Records are numbered starting with 1 .

Return values: 0 - no error detected

-1 - error detected

DEVELOPED BY: TrOY K. MoOre

DATE: $\quad 11 / 9 / 87$

REVISION NOTES:

12/4/87 TKM Added code to update cur_dif and next_dif with respect to the new target depth after a depth match occurred.

t-

*

Int select_recs ().

int found_count, read_bytes;

long Int seek_flag, rec_count:

float cur_dif, prev_dif, next_dif, target_depth, cur_depth;

vold error_handler ():

long Int lseek (): 


\section{Appendix K - BATT Source Code}

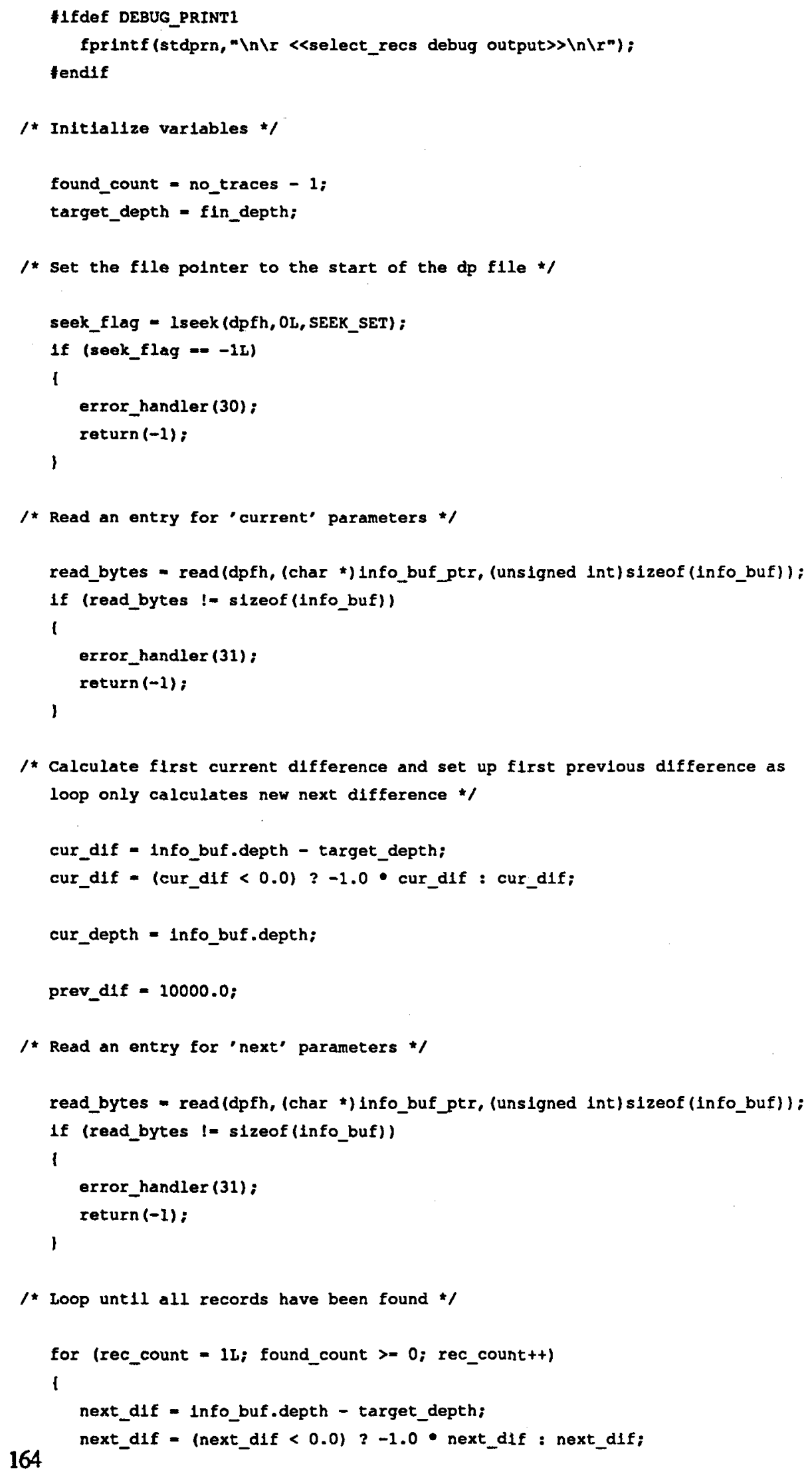




\section{Appendix K - BATT Source Code}

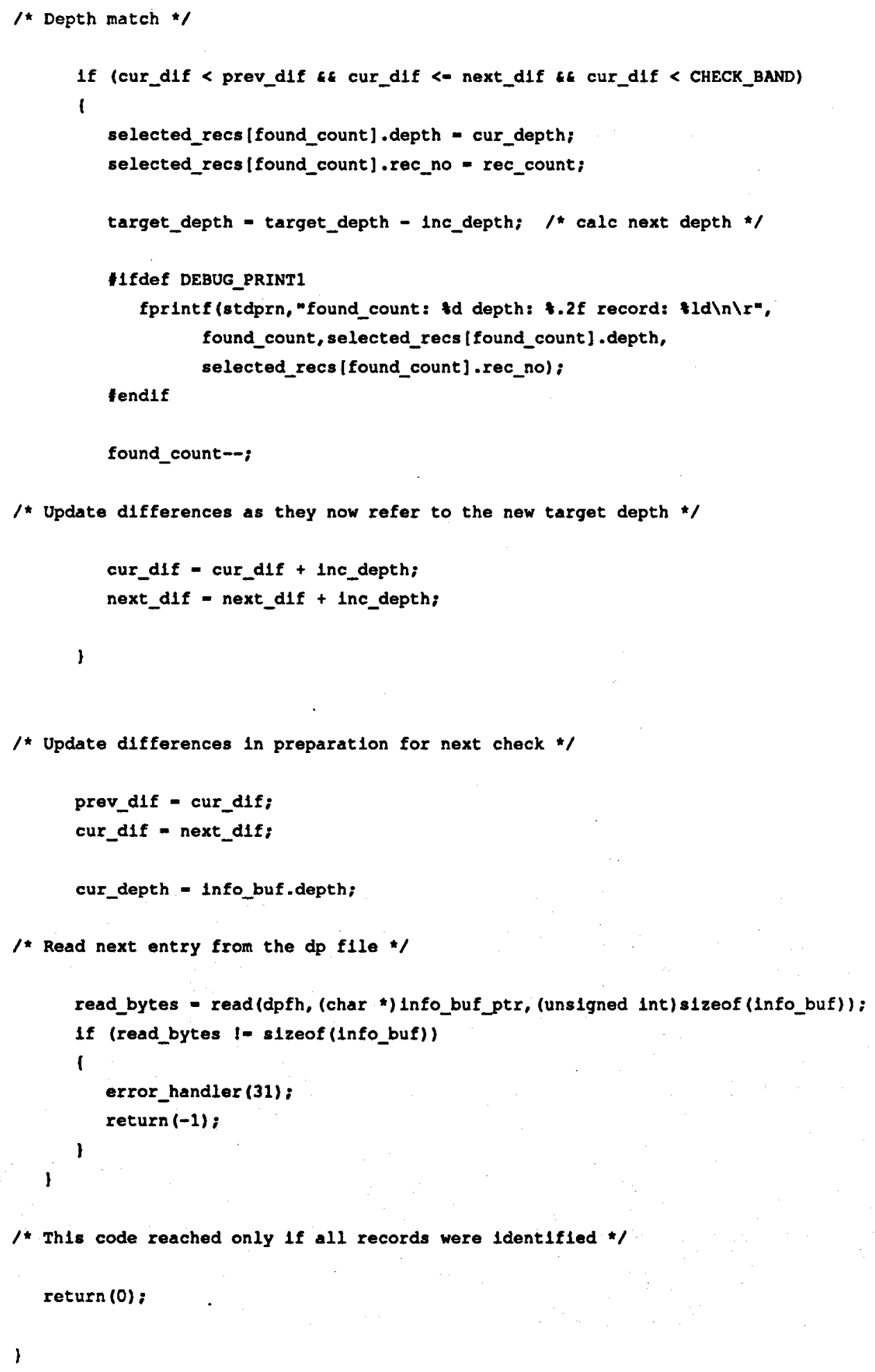




\section{Appendix K - BATT Source Code}

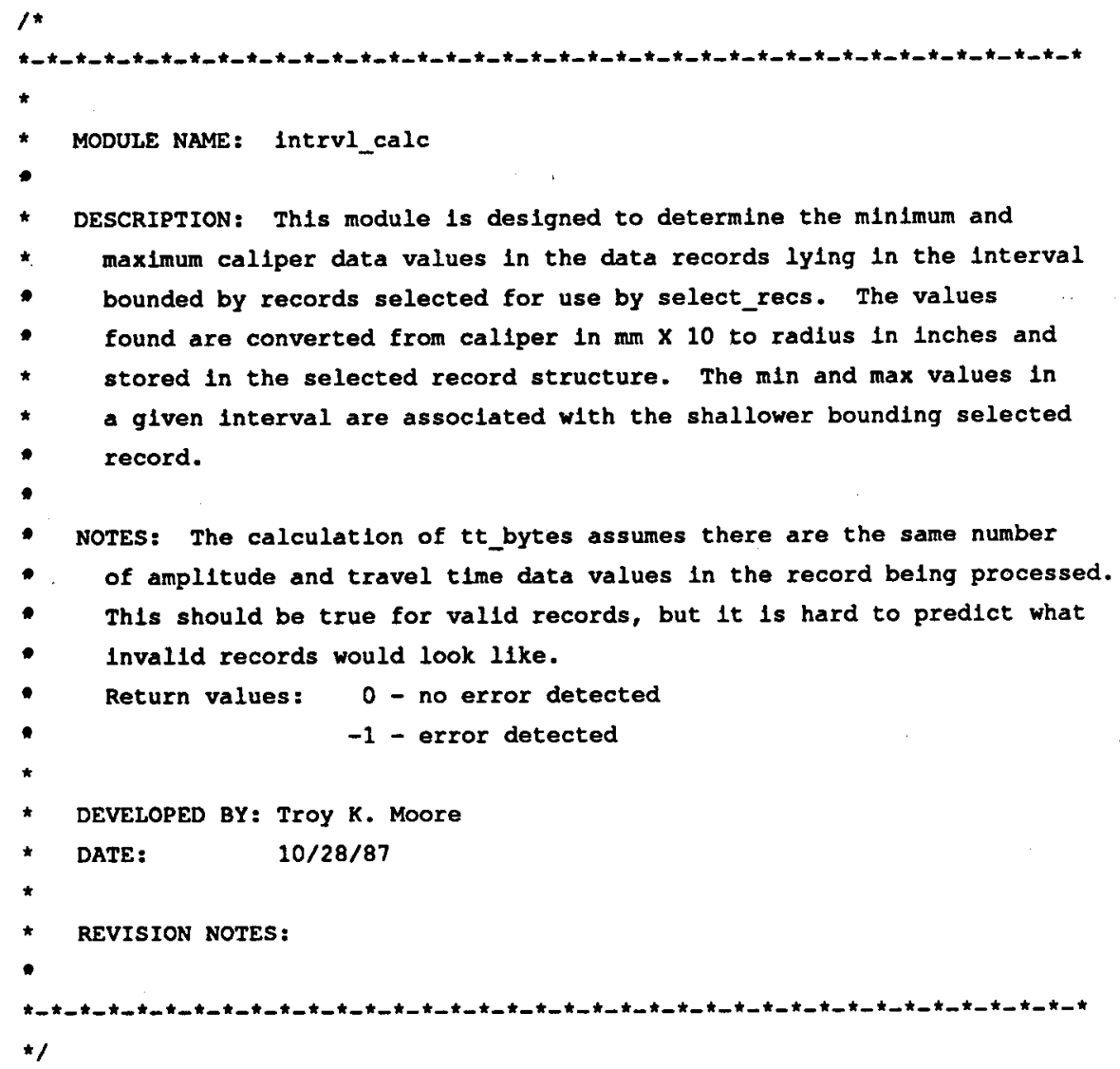




\section{Appendix K - BATT Source Code}

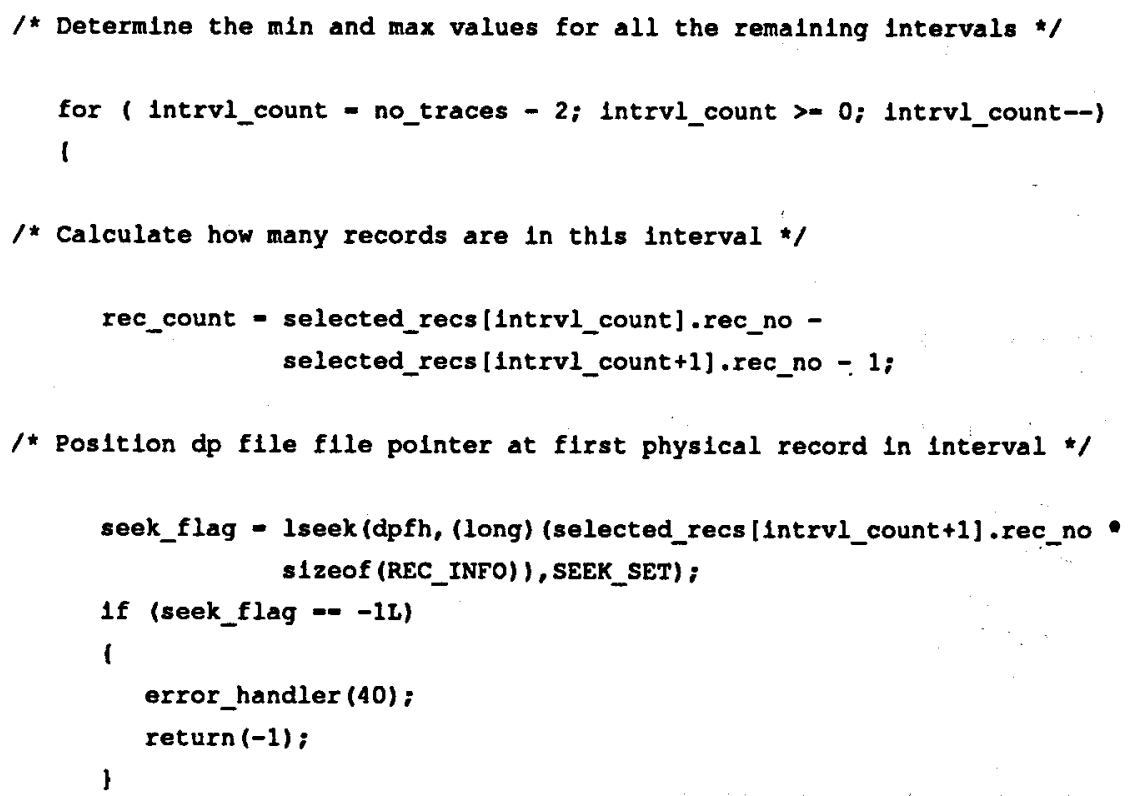




\section{Appendix K - BATT Source Code}

/* Read only the callper data */

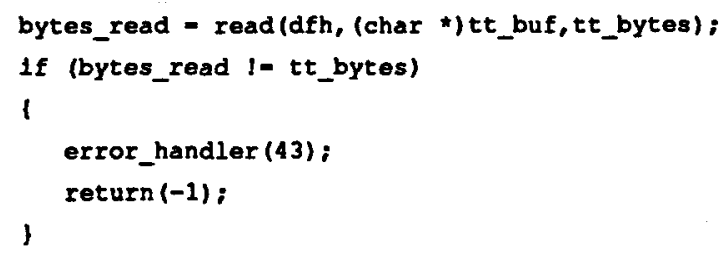




\section{Appendix K - BATT Source Code}

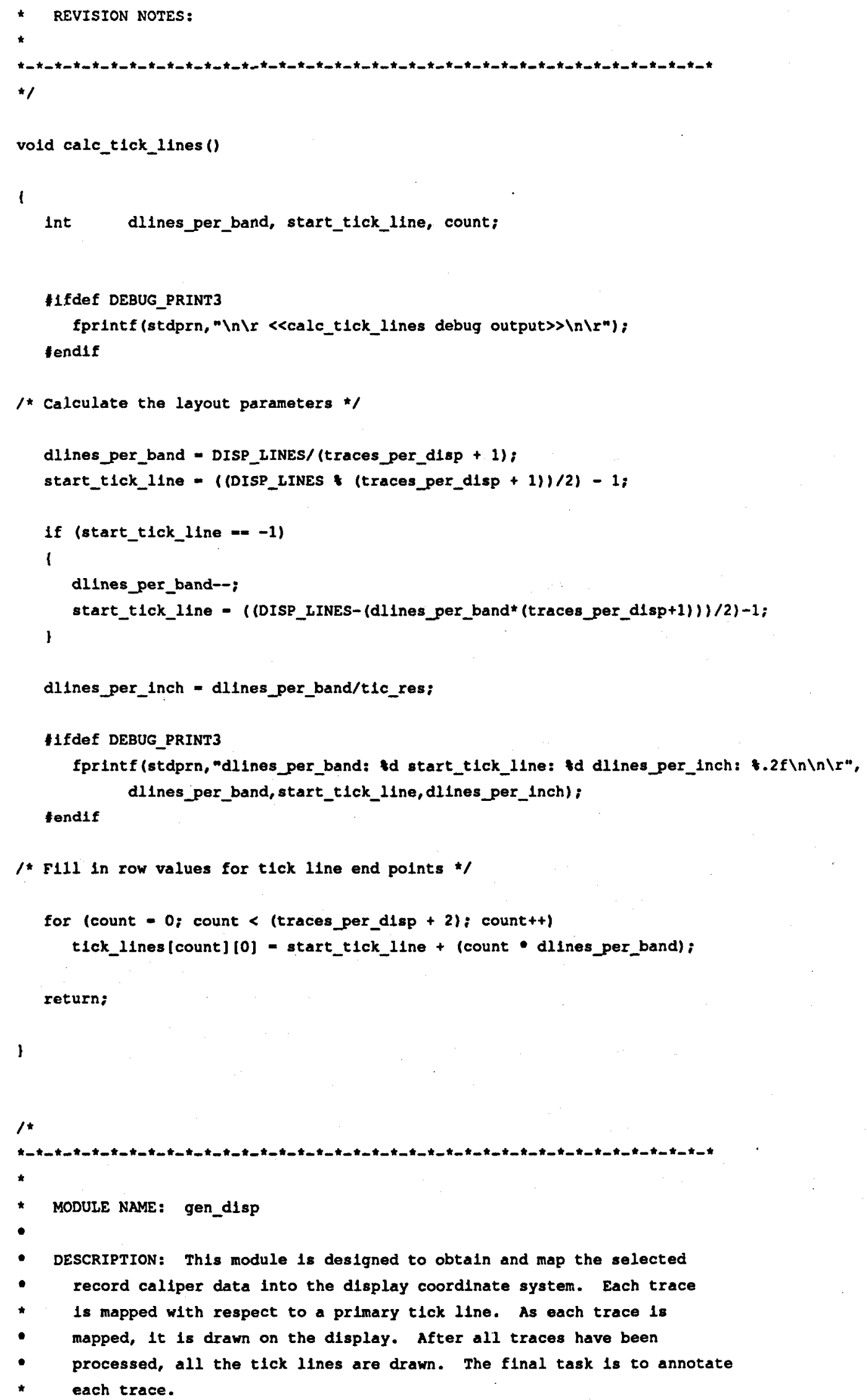
each trace. 


\section{Appendix K - BATT Source Code}

- NOTES

* Return values: 0 - no error detected

-

- DEVELOPED BY: Troy K. Moore

- DATE: $11 / 9 / 87$

-

- REVISION NOTES:

-

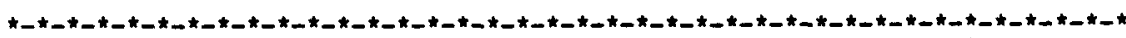

$\star /$

int gen_disp (loop_count)

int loop_count:

1 trace_l1mit, trace_count, srec_index, bytes_read, plxel_count: start_col, start_row, draw_count, disp_limit, ret_flag, tic_offset; stra_count, strb_count, strc_count, strd_count; stra[15], strb[15], strc[15], strd[15];

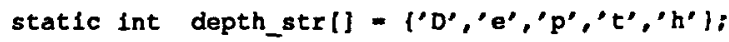

static int ref_str[] $=\left(R^{\prime},{ }^{\prime} e^{\prime}, f^{\prime}\right)$ :

static int min_str[] $=\left\{M^{\prime}, 1^{\prime}, n^{\prime}\right\}^{\prime} ;$

static int $\left.\max s_{-} \leq \|\right\}=\left\{{ }^{\prime} M^{\prime}, 0^{\prime} a^{\prime}, x^{\prime}\right\}$

unsigned int tt_bytes, skip;

unsigned int $t t$ _buf[POINTS_PER_TRACE];

long seek_flag;

double Inch_rad;

vold error_handler();

Int doub_convrt ():

long lseek ():

1 Ifdef DEBUG_PRINT4

fprintf (stdprn, "In $\mid r<<g e n \_d 1 s p$ debug output $\left.\gg \mid n \backslash r^{n}\right)$;

tendif

$/ *$ select intensity for drawing the traces */

is_set_foreground (TRACE_INTENSITY):

$I^{*}$ Calculate how many traces to display *

trace_limit $=\left(100 p_{\text {_count }}+1<\right.$ no_disps $)$ ? traces_per_disp :

no_traces - (100p_count * traces_per_disp); 


\section{Appendix K - BATT Source Code}

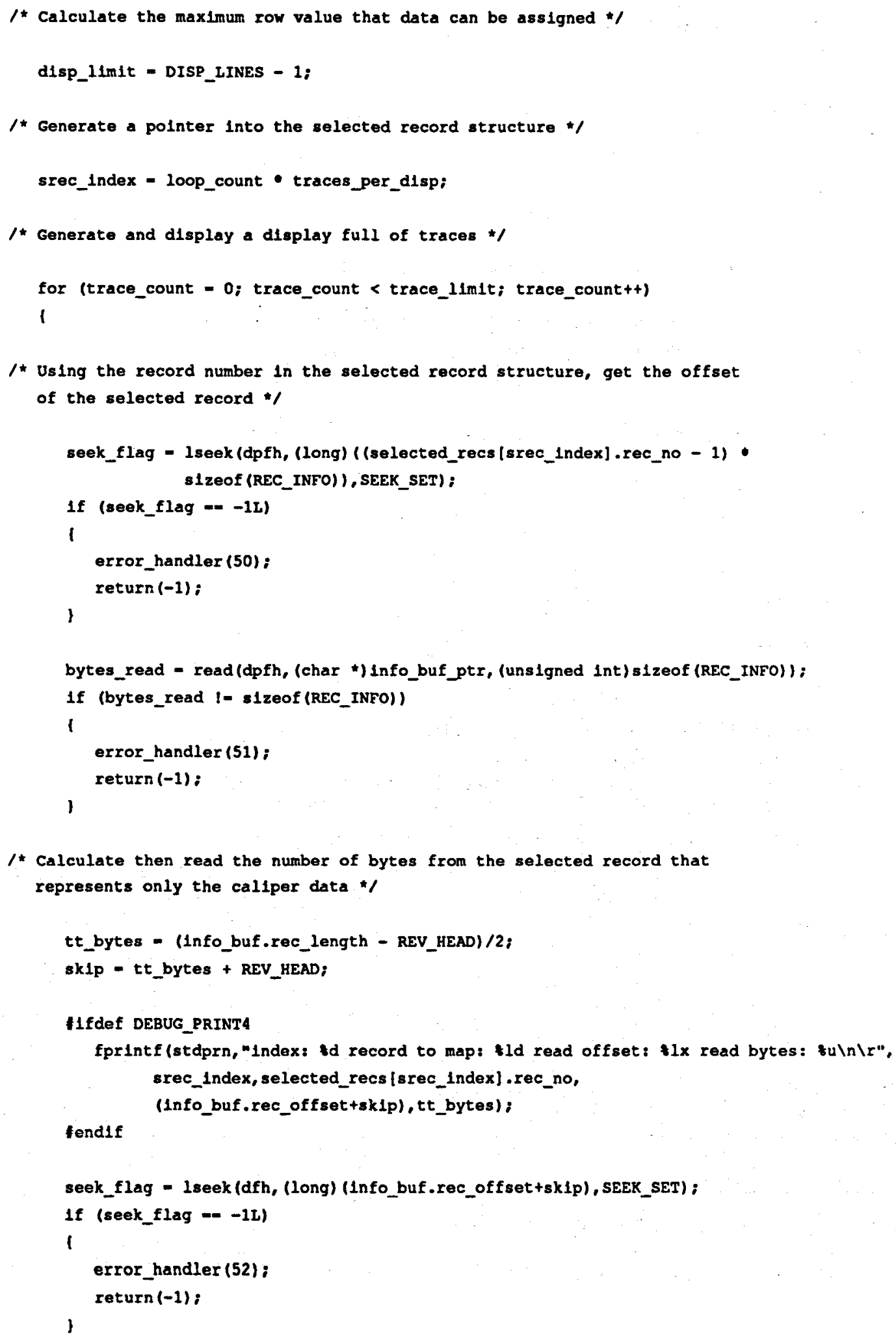




\section{Appendix K - BATT Source Code}

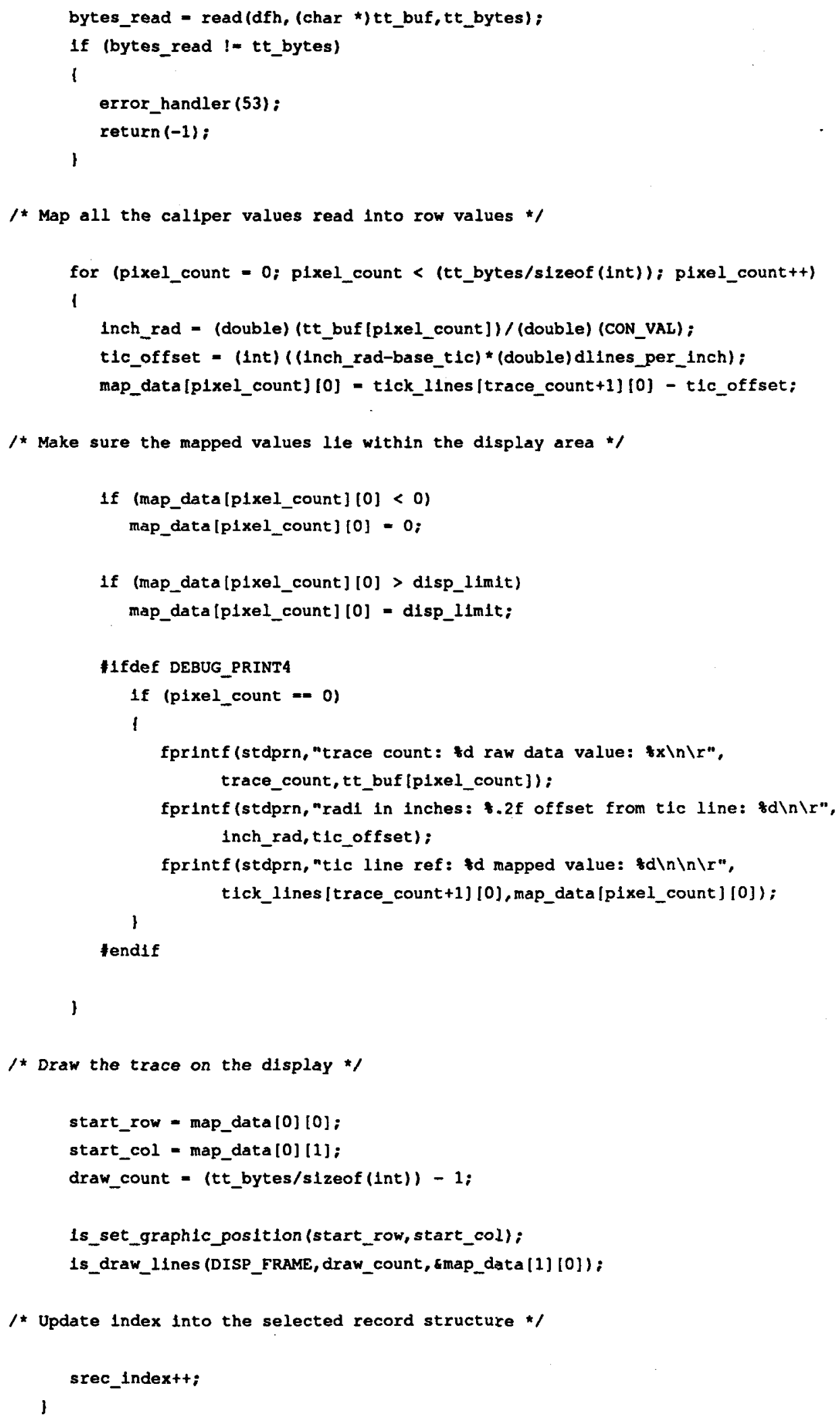




\section{Appendix K - BATT Source Code}

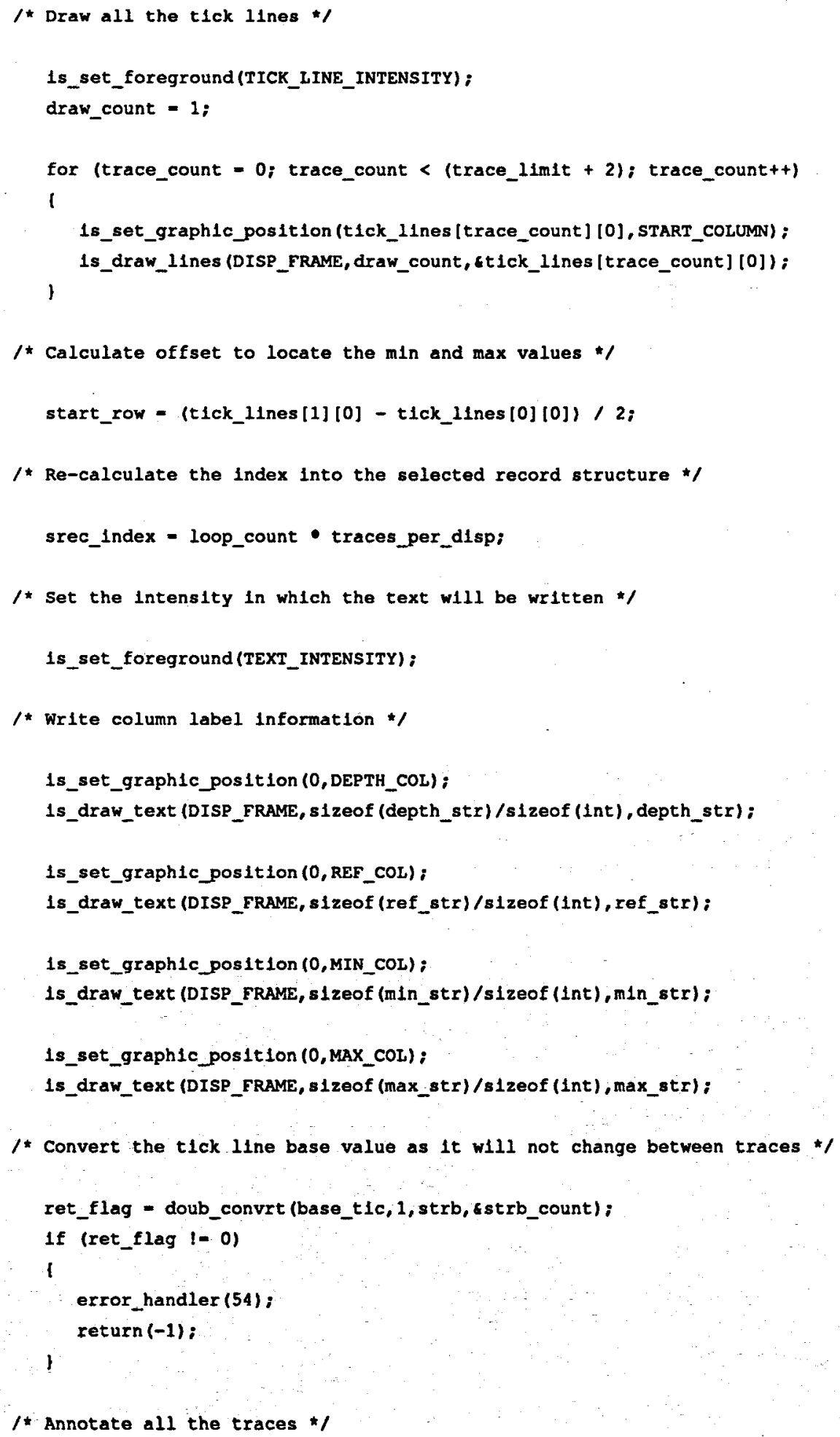




\section{Appendix K - BATT Source Code}

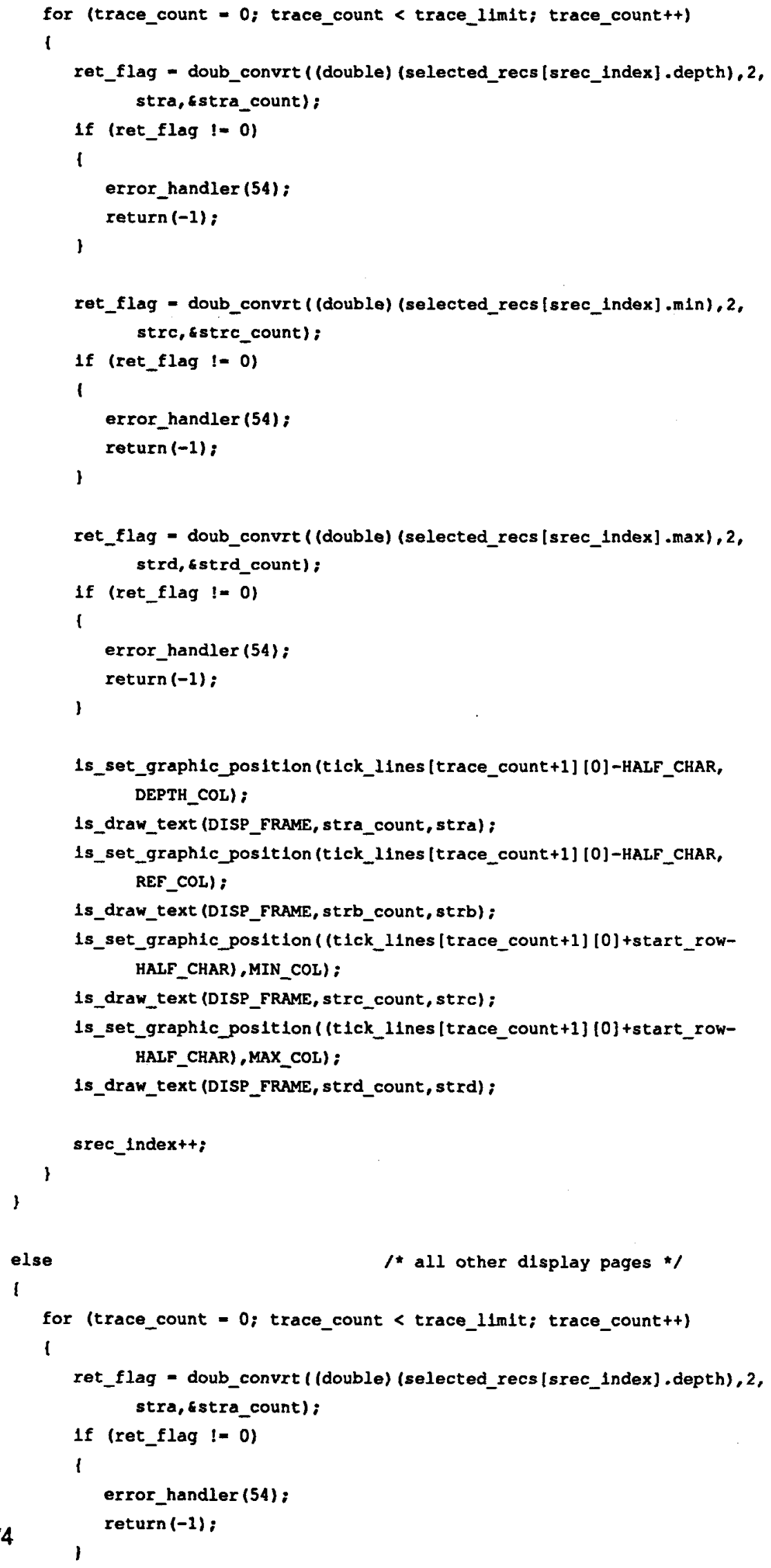




\section{Appendix K - BATT Source Code}

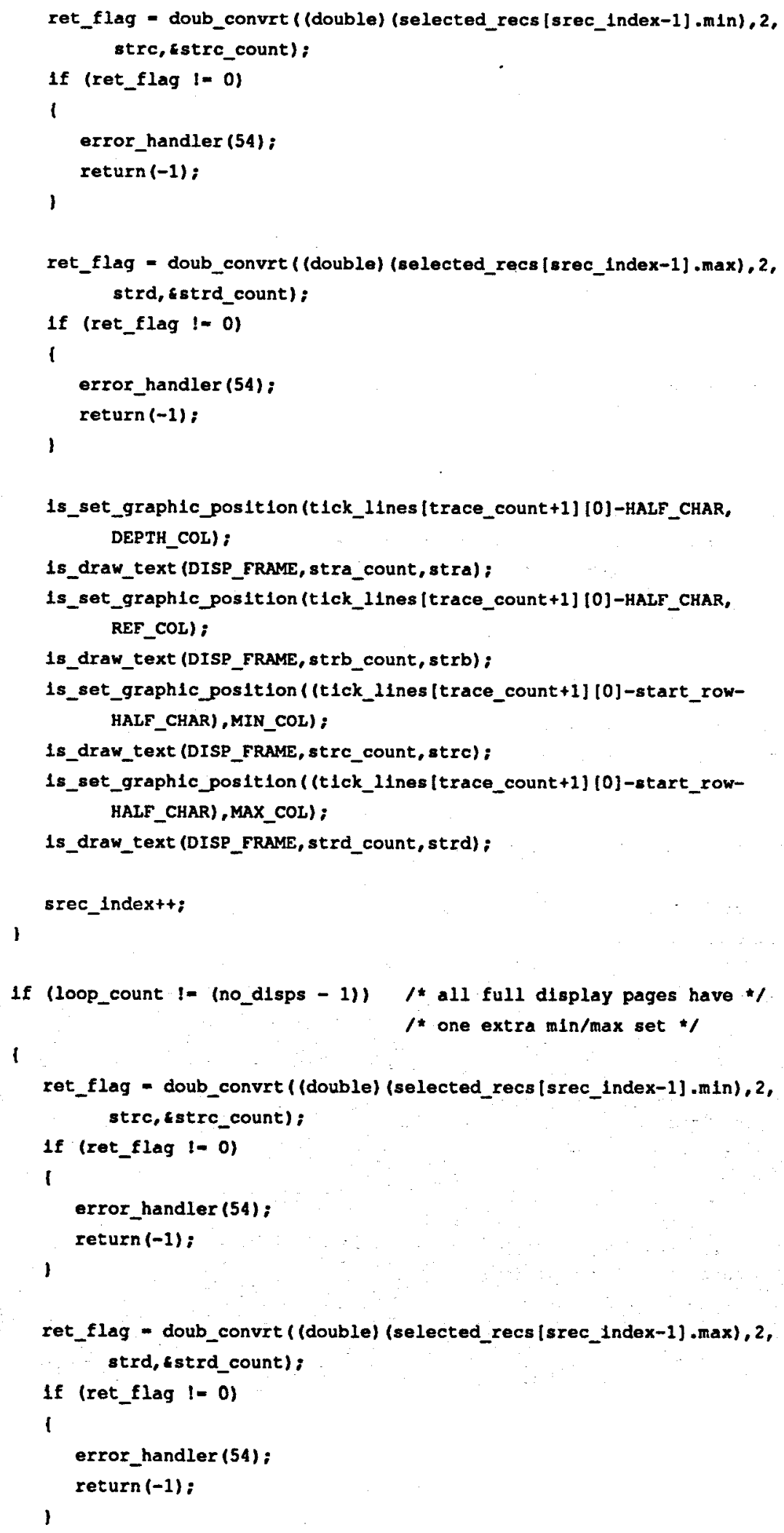




\section{Appendix K - BATT Source Code}

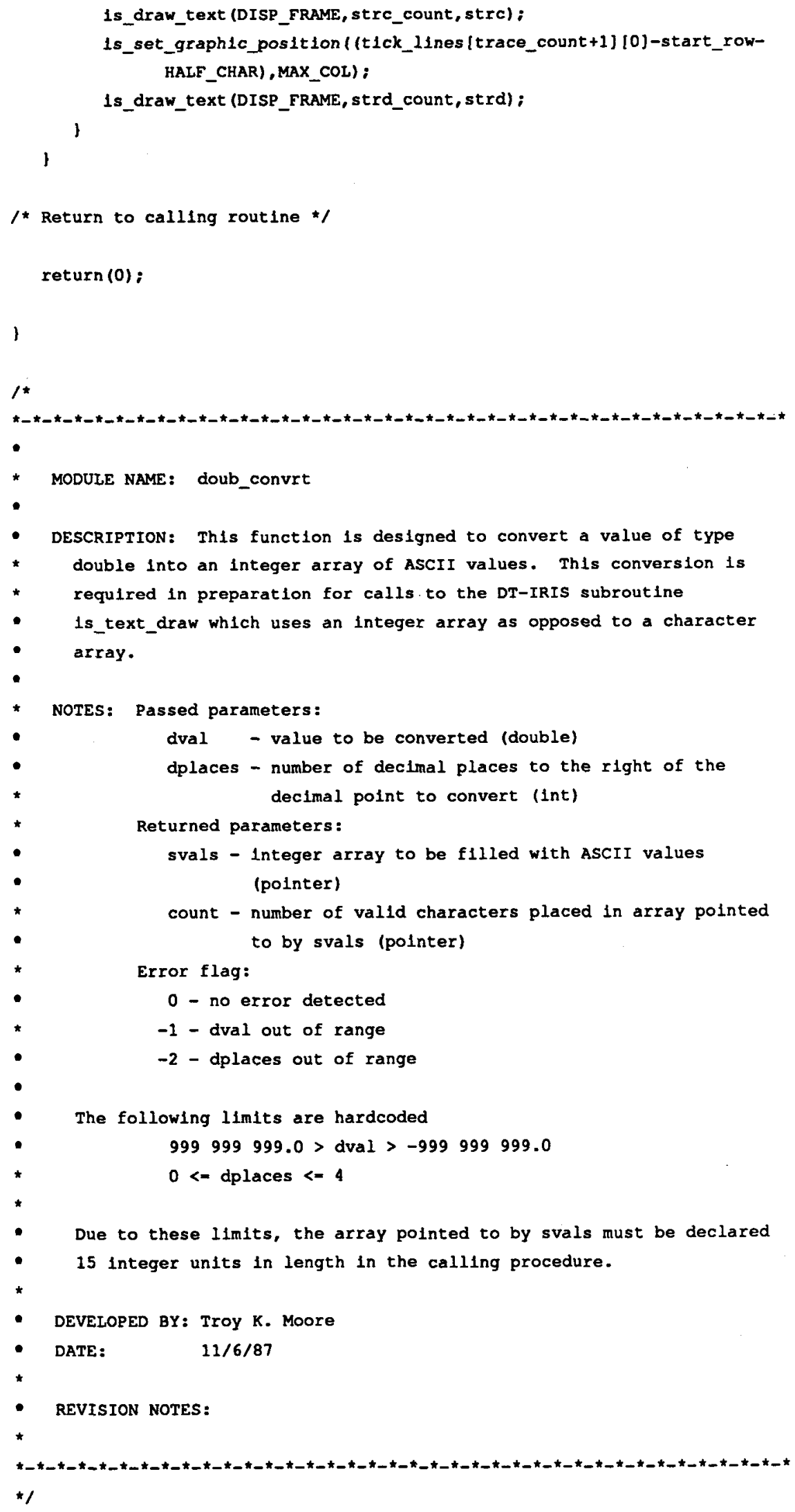




\section{Appendix K - BATT Source Code}

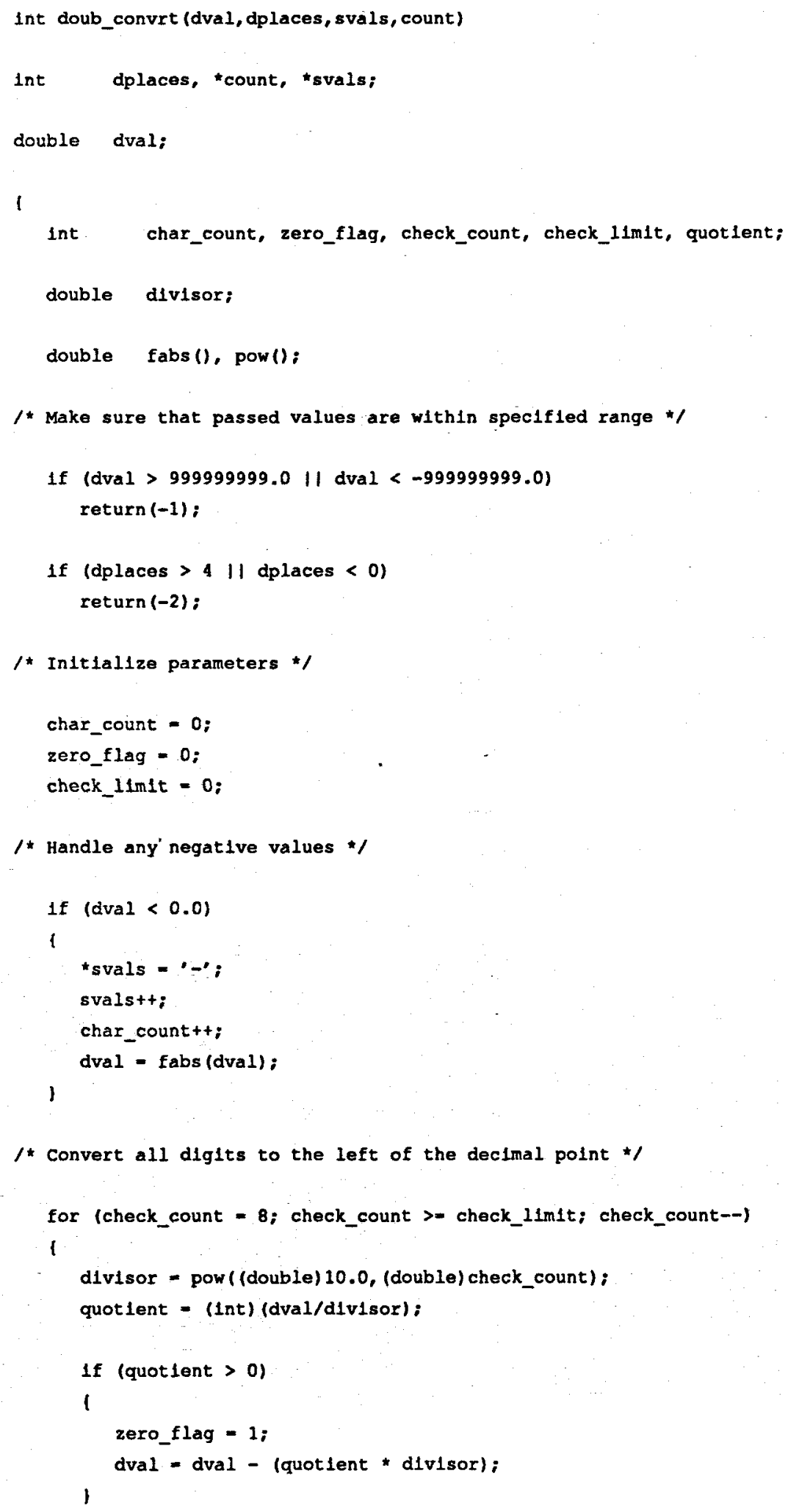




\section{Appendix K - BATT Source Code}

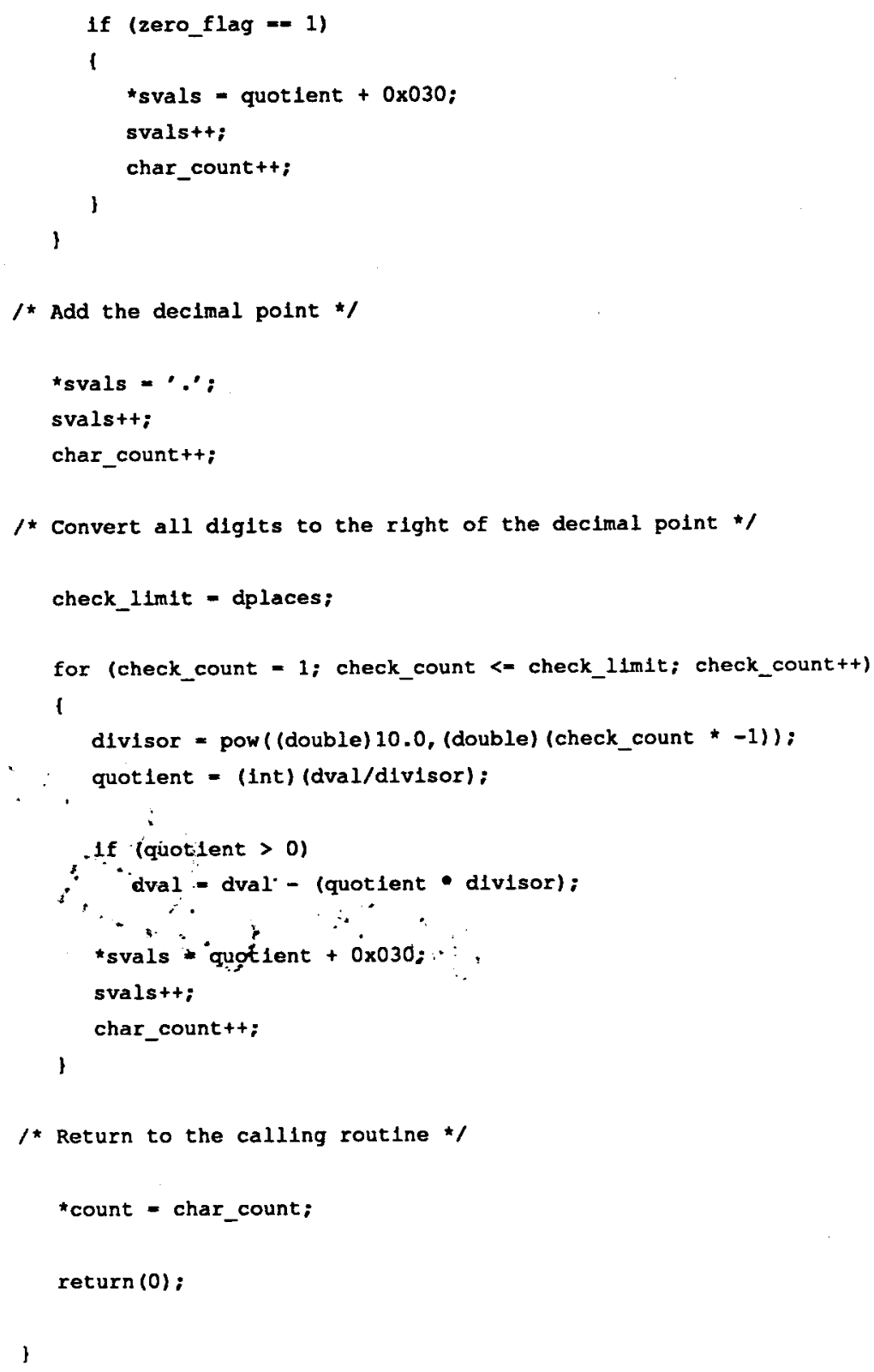

